\title{
Biomarkers in non-small cell lung cancer
}

Citation for published version (APA):

Botelho de Carvalho, S. (2016). Biomarkers in non-small cell lung cancer: imaging and Blood. [Doctoral Thesis, Maastricht University]. https://doi.org/10.26481/dis.20160630sb

Document status and date:

Published: 01/01/2016

DOI:

10.26481/dis.20160630sb

Document Version:

Publisher's PDF, also known as Version of record

\section{Please check the document version of this publication:}

- A submitted manuscript is the version of the article upon submission and before peer-review. There can be important differences between the submitted version and the official published version of record.

People interested in the research are advised to contact the author for the final version of the publication, or visit the DOI to the publisher's website.

- The final author version and the galley proof are versions of the publication after peer review.

- The final published version features the final layout of the paper including the volume, issue and page numbers.

Link to publication

\footnotetext{
General rights rights.

- You may freely distribute the URL identifying the publication in the public portal. please follow below link for the End User Agreement:

www.umlib.nl/taverne-license

Take down policy

If you believe that this document breaches copyright please contact us at:

repository@maastrichtuniversity.nl

providing details and we will investigate your claim.
}

Copyright and moral rights for the publications made accessible in the public portal are retained by the authors and/or other copyright owners and it is a condition of accessing publications that users recognise and abide by the legal requirements associated with these

- Users may download and print one copy of any publication from the public portal for the purpose of private study or research.

- You may not further distribute the material or use it for any profit-making activity or commercial gain

If the publication is distributed under the terms of Article $25 \mathrm{fa}$ of the Dutch Copyright Act, indicated by the "Taverne" license above, 


\section{Biomarkers in non-small cell lung cancer Imaging and Blood}

Sara João Carvalho

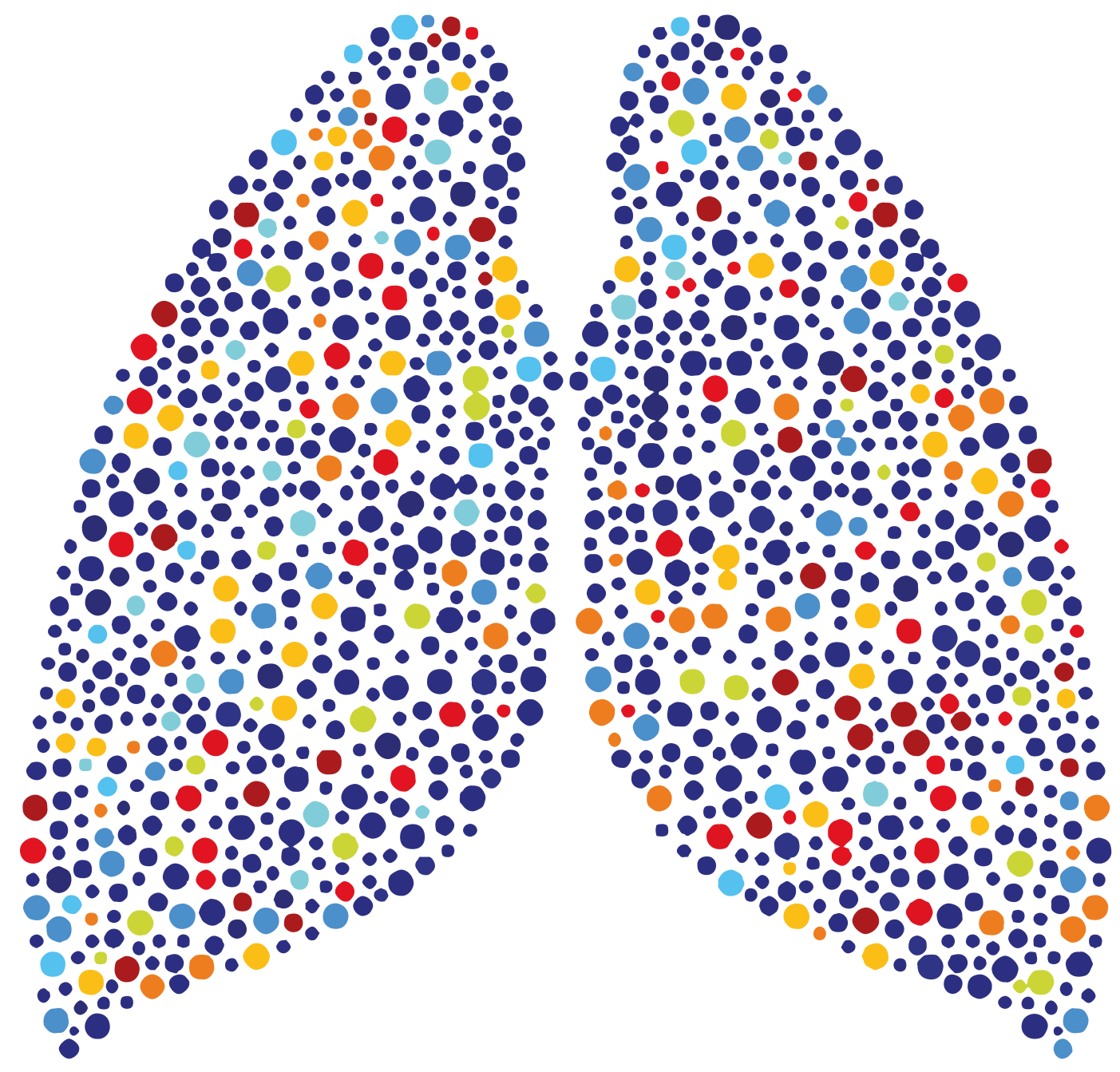


Cover Design by Nélson Vilhena

(C) copyright Sara Carvalho, Maastricht 2016

Printing: Datawyse | Universitaire Pers Maastricht

ISBN 9789461595744

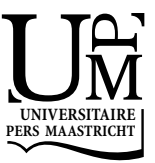




\title{
Biomarkers in non-small cell lung cancer Imaging and Blood
}

\author{
Dissertation by
}

Sara João Botelho de Carvalho

\begin{abstract}
To obtain a doctoral degree at Maastricht University, on the authority of the Rector Magnificus Prof. Dr. L.L.G. Soete, in accordance with the decision of the Board of Deans.

to be defended in public on

Thursday June $30^{\text {th }}, 2016$ at 12:00 hours
\end{abstract}




\section{Promotor}

Prof. Dr. Philipe Lambin

\section{Co-Promotor}

Dr. Cary Oberije

Dr. Ir. Wouter van Elmpt

\section{Assessment Committee}

Prof. Dr. Ir. Frank Verhaegen (chairman)

Prof. Dr. Iréne Buvat, CEA Université Paris Sud, Orsay, France

Dr. Eline Kooi

Prof. Dr. Anne-Marie Dingemans

Prof. Dr. Nuno Ferreira, University of Coimbra, Portugal

The work presented in this thesis was made possible by the financial support of: QulC-ConCePT project, the Dutch Technology Foundation STW (grant $n^{\circ} 10696$ DuCAT), CTMM framework (AIRFORCE project, grant 030103), EU 7th framework programme (EURECA, ARTFORCE - $n^{\circ}$ 257144, REQUITE - $n^{\circ}$ 601826), SME Phase 2 (EU proposal 673780 - RAIL), Kankeronderzoekfonds Limburg from the Health Foundation Limburg and the Dutch Cancer Society (KWF UM 2008-4210, KWF UM 2009-4454). 


\section{Contents}

Introduction

Chapter 1 General introduction and outline of the thesis

Chapter 2 "Rapid Learning health care in oncology" - An approach towards decision support systems enabling customised radiotherapy

Chapter 3 Radiomics: Extracting more information from medical images using advanced feature analysis

Imaging Biomarkers - Radiomics

Chapter 4 Prognostic value of metabolic metrics extracted from baseline positron emission tomography images in non-small cell lung cancer

Chapter 5 Stability of FDG-PET Radiomics features: An integrated analysis of test-retest and inter-observer variability

Chapter 6 Early variation of FDG-PET Radiomics features in NSCLC is related to overall survival - The "Delta Radiomics" concept

Chapter 7 FDG-PET Radiomics of metastatic lymph nodes and primary tumour in NSCLC - A prospective study externally validated

\section{Blood Biomarkers}

Chapter 8 Prognostic value of blood-biomarkers related to hypoxia, inflammation, immune response and tumour load in non-small cell lung cancer - A survival model with external validation

\section{Discussion}

Chapter 9 General discussion, concluding remarks, and future perspectives

Societal Impact and Valorisation

Acknowledgments

Curriculum Vitae 

Chapter

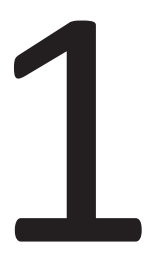

General introduction and outline of the thesis 


\section{INTRODUCTION}

World Health Organization positions cancer, with an estimated 8.2 million of annual deaths (in 2012), and expected to increase by $70 \%$ in the next two decades, as a leading cause of death [1]. In Oncology, disease's exponential spread and fast development dictates that depending exclusively on clinical trial evidences is becoming a nonsustainable nor efficient way to deal with poor outcomes, thereupon rapid-learning methodologies have been gaining increasing attention [2]. Rapid-learning health care models are designed to routinely and iteratively learn from the continuously in grow databases, that in return are renovated and improved. The "learning" phase encompasses high-quality data collection and analysis, aimed to generate evidences - clinical prognostic tools, then to be implemented and evaluated in clinical practice (Figure 1), further to generate new hypothesis, to be investigated by combination of old and newly available data leading to a complete cycle repetition [3]. As a result, quality controlled data collection routines are to be performed, as to improve robustness on clinical prognostic tools development, correct validation procedures, studies replication and possible extensions (Figure 1) [4].

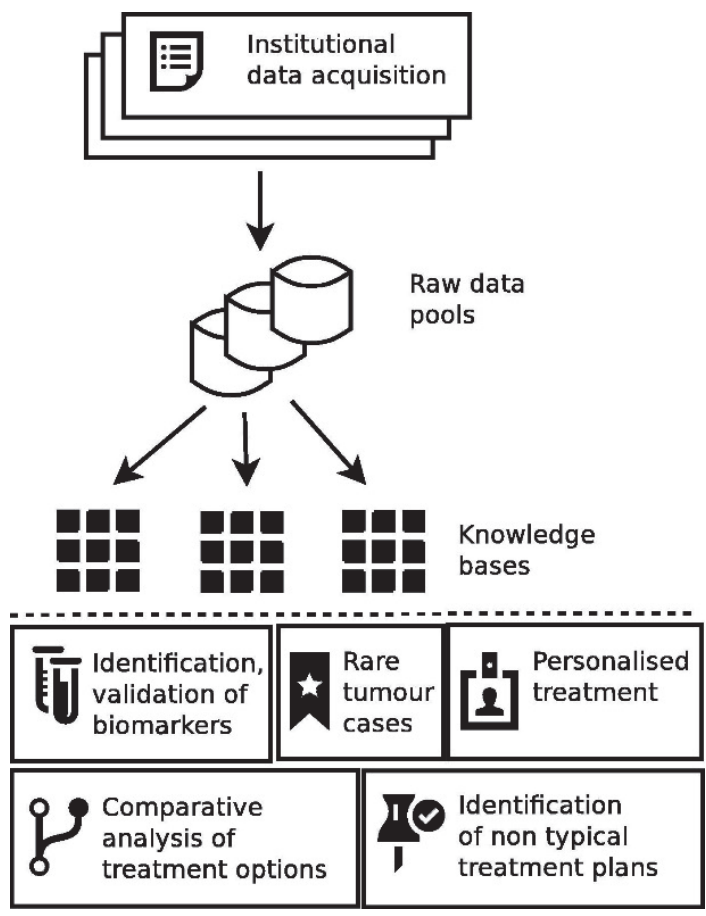

Figure 1 - From data to clinical prognostic tools: applicationspecific prediction models or knowledge bases are generated, based on institutional data (from [4]). 
The ever-growing information availability is aimed to provide clinicians with means to perform a deeper informed decision, aligned with the promise of an individualized and personalized prognostication $[2,5]$. However, the great increase and synergetic combination of diagnostic tools and treatment modalities, standard and pathologic factors, biological, genetic, and other molecular characteristics of both patient and tumour's, are becoming unbearable to the limited human cognitive capacity, aggravating optimal decisions for the individual patient $[3,6]$. Clinical prognostic tools, generating evidence through retrospective analysis of existing data as well as from data of prospective studies, are expected to actively contribute to a superior personalized medicine, implying a good study design and analysis approaches for development and particularly validation is followed [5].

\section{OBJECTIVE OF THE THESIS}

With 1.59 million reported deaths in 2012, lung cancer is the most common cause of cancer death, being the most common cancer type among men and third most prevalent in women (after breast and colorectal cancer). Two distinct forms of lung cancer are identifiable: small cell (SCLC) and non-small cell lung cancer (NSCLC), with the latter accounting for about $85 \%$ of the total number of cases, and being the focus of this thesis.

Clinical prognostic tools for an individualized and personalized medicine are a primary goal in lung cancer. For this purpose, identification of tumour traits to be incorporated into clinical prognostic tools resumes the main motivation of this work. However, tissue sampling represents an extra burden for lung cancer patients and often, biopsies are not possible to perform. Therefore, and accounting for this limitation, this thesis aimed to identify and validate non-invasive sources of information, able to provide with prognostic and predictive information, based on:

1. Imaging biomarkers: derived from ${ }^{18} \mathrm{~F}$-fluorodeoxyglucose (FDG) patterns of metabolism assessed by positron emission tomography (PET) imaging of primary tumour and metastatic lymph nodes, before radiotherapy delivery, and extended with early variation of these metrics during treatment.

2. Blood-biomarkers: circulating oncoproteins related to hypoxia, inflammation, tumour load and immune response. 


\section{OUTLINE OF THIS THESIS}

\section{1. introduction}

This thesis is divided into four sections. A general introduction is presented in this Chapter 1 , and further extended with an overview of rapid learning methods for radiotherapy in Chapter 2 and a concept paper on the imaging biomarkers (Radiomics) on Chapter 3. Radiomics comprises the extraction of high-throughput of imaging descriptors from a defined region-of-interest (ROI), further to be analysed in accordance to a pre-defined outcome, using sophisticated statistics and modelling techniques. Following chapters elaborate on the clinical applicability of this premise, by investigating data cases, with external and independent validation. A graphical representation of thesis contents from Chapter 4 through 8 is depicted in Figure 2.

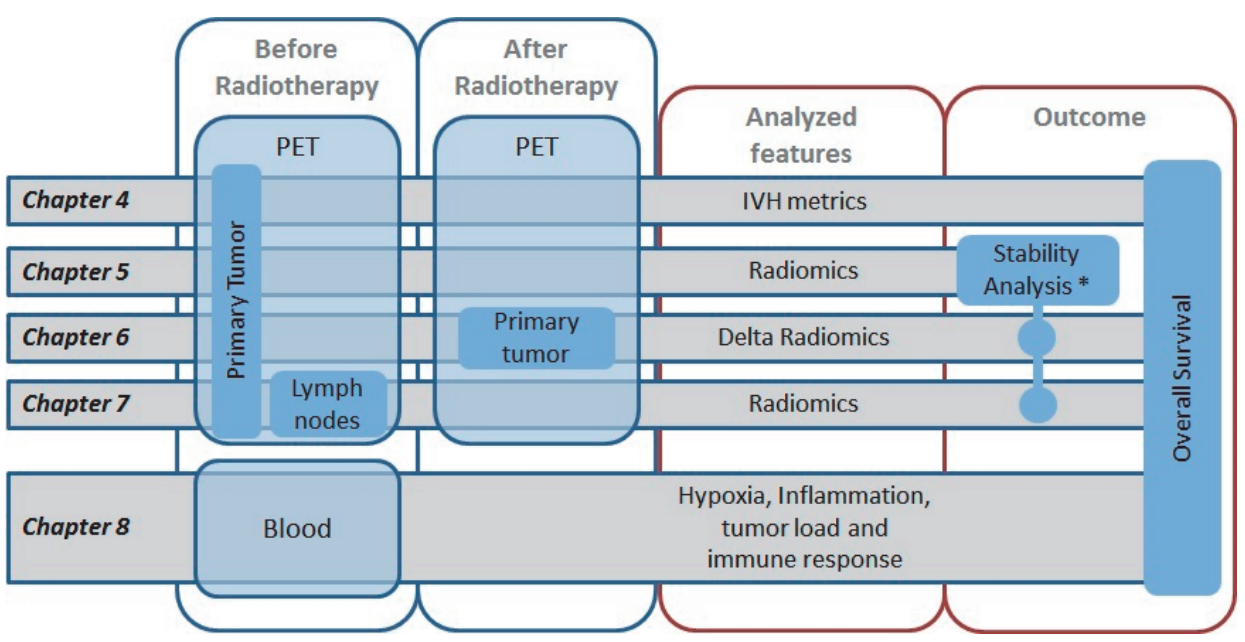

Figure 2 - Overview of thesis contents. Chapters 4 to 7 refer to imaging biomarkers; Chapter 8 describes the work conducted for blood-biomarkers. $\left({ }^{*}\right)$ Results of a stability analysis conducted in Chapter 5 , are used in the two sequent chapters for stable and robust features identification, prior to analysis.

\section{Imaging biomarkers - Radiomics}

Chapter 4 presents an analysis of the intensity-volume histograms (IVH) descriptors (analogous to the dose-volume histogram concept, but using SUV distribution instead) and comparison with the most commonly used metrics derived from PET imaging, analysed on a dataset of 220 NSCLC patients. Images analysed in this thesis were first discretized with a bin width of 0.5 (standard uptake value (SUV)), according to:

$$
I_{D}(x)=\left\lceil\frac{I(x)}{0.5}\right\rceil-\min \left(\left\lceil\frac{I(x)}{0.5}\right\rceil\right)+1
$$


Where $I$ is the original image, $I(x)$ represent the SUV of voxel $x$, and $I_{D}$ is the resulting discretized image [7].

In Chapter 5 a stability analysis of all the imaging biomarkers analysed is presented. These imaging biomarkers, often mentioned as Radiomics features along this thesis, include first order statistic, shape and size descriptors, texture descriptors (including grey-level co-occurrence - GLCM, grey-level run-length - RLGL and grey-level size zone matrices - GLSZM) and intensity-volume histograms (IVH). Stability analysis comprised the investigation of the robustness of these features in a test-retest scenario conducted in 11 NSCLC patients, and across independent and manual delineations of five radiation oncologists blinded to each other's segmentations on pre-radiotherapy PET-CT scans of 23 NSCLC patients. Results and main conclusions presented in this chapter were used in the next two for stable and robust features identification prior to further analysis. An appendix to this chapter presents with mathematical formulations for the analysed Radiomics features.

Chapter 6 resumes the hypothesis that early percentage variation of Radiomics features derived from the primary lesion are predictive factors for overall survival assessment of NSCLC. For this purpose, a multivariable model was derived on a dataset $(n=54)$ including a PET scan acquired prior to radiotherapy delivery and repeated early during treatment. Findings were validated in two independent datasets ( $n=32$ and 26) with similar imaging acquisition protocol.

Lung cancer cells can enter lymphatic vessels and grow in lymph nodes around the bronchi and in the mediastinum. The nodal stage is highly related to disease progression and capability to metastasize and therefore determines treatment choice. In Chapter 7 the imaging analysis that so far was conducted solely for the primary tumour is extended to the metastatic lymph nodes, following the hypothesis that these may enclose additional prognostic value. A multivariable model is developed based on the preradiotherapy PET signal of a large dataset of nodal positive patients $(n=262)$ and further validated in an independent dataset of NSCLC patients $(n=50)$.

\section{Blood-biomarkers}

Chapter 8 presents the work conducted in blood-biomarkers and the extension of a clinical model comprising World Health Organization performance-status, forced expiratory volume in 1 second, number of positive lymph node stations, and gross tumour volume (and sequent validation), by selecting amongst hypoxia [osteopontin (OPN) and carbonic anhydrase IX (CA-IX)], inflammation [interleukin 6 (IL-6), IL-8, and C-reactive protein (CRP)], and tumour load markers [carcinoembryonic antigen (CEA) and cytokeratin fragment (CYFRA 21-1)]. This chapter was finalized with an extension of this validated model by selecting amongst new markers, including immunological ones: alpha-2-macroglobulin $(\alpha 2 M)$, serum IL-2 receptor (sIL2R), toll-like receptor 4 (TLR4), and vascular endothelial growth factor (VEGF). 
CHAPTER 1

4. General discussion and future perspectives

Final Chapter 9 is dedicated to a general conclusion, summarizing the main findings of each chapter and presenting the potential next steps following the present work. 


\section{REFERENCES}

[1] Stewart BW, Wild CP. World Cancer Report 2014. IARC.

[2] Lambin P, Zindler J, Vanneste B, van de Voorde L, Jacobs M, Eekers D, et al. Modern clinical research: How rapid learning health care and cohort multiple randomised clinical trials complement traditional evidence based medicine. Acta oncologica (Stockholm, Sweden). 2015 Oct;54(9):1289-1300.

[3] Abernethy AP, Etheredge LM, Ganz PA, Wallace P, German RR, Neti C, et al. Rapid-learning system for cancer care. Journal of clinical oncology : official journal of the American Society of Clinical Oncology. 2010 Sep 20;28(27):4268-4274.

[4] Skripcak T, Belka C, Bosch W, Brink C, Brunner T, Budach V, et al. Creating a data exchange strategy for radiotherapy research: towards federated databases and anonymised public datasets. Radiotherapy and oncology : journal of the European Society for Therapeutic Radiology and Oncology. 2014 Dec;113(3):303-309.

[5] Mahar AL, Compton C, McShane LM, Halabi S, Asamura H, Rami-Porta R, et al. Refining Prognosis in Lung Cancer: A Report on the Quality and Relevance of Clinical Prognostic Tools. Journal of thoracic oncology : official publication of the International Association for the Study of Lung Cancer. 2015 Nov;10(11):15761589.

[6] Oberije C, Nalbantov G, Dekker A, Boersma L, Borger J, Reymen B, et al. A prospective study comparing the predictions of doctors versus models for treatment outcome of lung cancer patients: a step toward individualized care and shared decision making. Radiotherapy and oncology : journal of the European Society for Therapeutic Radiology and Oncology. 2014 Jul;112(1):37-43.

[7] Leijenaar RT, Nalbantov G, Carvalho S, van Elmpt WJ, Troost EG, Boellaard R, et al. The effect of SUV discretization in quantitative FDG-PET Radiomics: the need for standardized methodology in tumour texture analysis. Scientific reports. 2015;5:11075. 



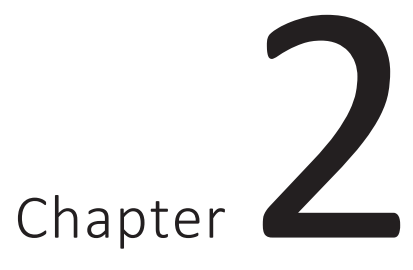

\section{"Rapid Learning health care in oncology" An approach towards decision support systems enabling customised radiotherapy}

Published in: Radiotherapy and Oncology, 109 (1), 2013 Oct: 159-164

\footnotetext{
'Rapid Learning health care in oncology' - An approach towards decision support systems enabling customised radiotherapy'

Philippe Lambin, Erik Roelofs, Bart Reymen, Emmanuel Rios Velazquez, Jeroen Buijsen, Catharina M.L. Zegers, Sara Carvalho, Ralph T.H. Leijenaar, Georgi Nalbantov, Cary Oberije, M. Scott Marshall, Frank Hoebers, Esther G.C. Troost, Ruud G.P.M. van Stiphout, Wouter van Elmpt, Trudy van der Weijden, Liesbeth Boersma, Vincenzo Valentini, Andre Dekker
} 


\section{ABSTRACT \\ Introduction}

An overview of the Rapid Learning methodology, its results, and the potential impact on radiotherapy.

\section{Methods and Results}

Rapid Learning methodology is divided into four phases. In the data phase, diverse data are collected about past patients, treatments used, and outcomes. Innovative information technologies that support semantic interoperability enable distributed learning and data sharing without additional burden on health care professionals and without the need for data to leave the hospital. In the knowledge phase, prediction models are developed for new data and treatment outcomes by applying machine learning methods to data. In the application phase, this knowledge is applied in clinical practice via novel decision support systems or via extensions of existing models such as Tumour Control Probability models. In the evaluation phase, the predictability of treatment outcomes allows the new knowledge to be evaluated by comparing predicted and actual outcomes.

\section{Conclusion}

Personalised or tailored cancer therapy ensures not only that patients receive an optimal treatment, but also that the right resources are being used for the right patients. Rapid Learning approaches combined with evidence based medicine are expected to improve the predictability of outcome and radiotherapy is the ideal field to study the value of Rapid Learning. The next step will be to include patient preferences in the decision making. 


\section{INTRODUCTION}

Tailored cancer therapies, in which specific information about patients and tumours is taken into account during treatment decisions, are an important step forward from current population based therapy [1]. However, given the developments outlined below, it is becoming increasingly difficult to identify the best treatment for an individual cancer patient:

- Tumours and patients seem to be even less homogeneous than previously assumed, meaning the same treatments can have different outcomes in patients who have the same type of tumour. For instance, there are at least four molecular subtypes of breast cancer, each with very different outcomes [2]. Based on gene signatures various subgroups of tumours can be identified [3-8].

- The number of treatment options is increasing. For example, early stage prostate cancer can now be treated with conservative treatment, prostatectomy, external radiotherapy, stereotactic radiotherapy, LDR or HDR brachytherapy, high-intensity focused ultrasound, hormone therapy, combination therapies and so on. A different example is the recent rise of targeted therapies that are rapidly growing in numbers. Performing classic randomised trials to compare all new treatment options with the "gold standard" is becoming impossible by the current speed of innovation.

- The evidence for the right choice in an individual patient is inadequate. First, 'evidence-based medicine' and the ensuing guidelines always lag somewhat behind practice, particularly in highly technological, innovative and rapidly evolving fields such as radiotherapy. In addition, translating the results of clinical trials to the general patient population and environment is not straightforward, given the higher quality of care in clinical trials and the known selection bias (trials reach no more than $3 \%$ of cancer patients, in radiotherapy this figure is even lower) [9-11]. Finally, given the developments mentioned above - more treatment options and less homogeneous patient groups - the urgency to scaffold our treatment decisions with robust knowledge and the demand for evidence-based medicine is larger than ever.

- It is becoming more difficult to find the right evidence. Despite - or perhaps due to the fact that papers are being published in rapidly increasing numbers (e.g., as a radiation-oncologist specializing in lung cancer, has to read around eight articles per day to keep up with the literature [12]), it is difficult to match the characteristics of the individual patient to evidence from the literature and to evaluate the quality of that evidence 

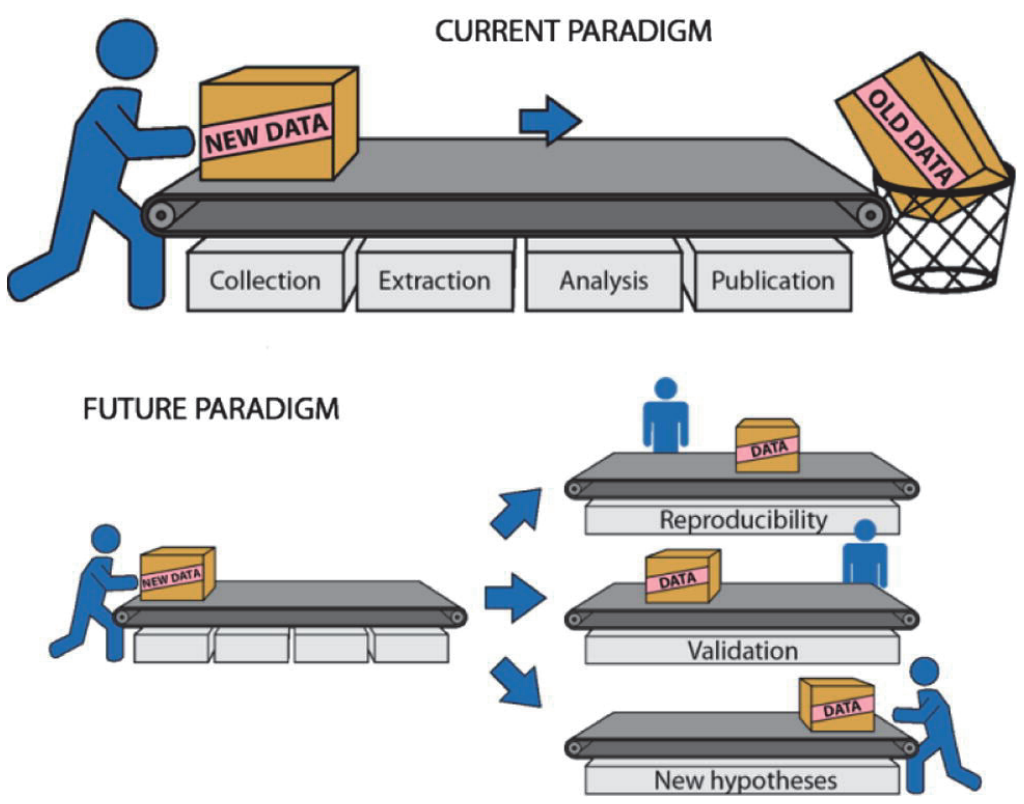

Figure 1 - Current paradigm versus future paradigm (modified from [13]).

The developments illustrated above have given rise to a search for an alternative to the elaborate consensus- and evidence-based guideline medicine format when it comes to making treatment decisions. The alternative discussed in this article is rapid learning [14]. Although it is known under various names, including Knowledge-driven Healthcare, Computer Assisted Theragnostics and Learning Intelligence Network, the basic idea in all cases is the (re)use of historical data from routine clinical practice for decisions concerning new patients or to test new hypothesis [15-20] (Figure 1). This has a number of obvious advantages, such as the large number of readily available patients and less selection bias compared to clinical trials. However, it also has some important disadvantages; for example, the quality of the data in clinical practice is much lower than in clinical trials [21]. There is a long very successful history of putting genomic data public and reusing them [3-8]. This paper provides an overview of the methods used in Rapid Learning, the initial results, and an outlook as to how the techniques involved may influence clinical radiotherapy.

\section{METHODS AND RESULTS}

Rapid Learning involves four phases (Figure 2) [14] which are continually iterated. In the data phase, data on past patients are collected, including their delivered treatments and outcomes. In the knowledge phase, knowledge is generated from these data. In the application phase, this knowledge is applied to clinical practice. In the final evaluation 
phase, the outcomes are evaluated, after which the first phase starts again. In every phase, external knowledge (e.g., from clinical trials) is used to optimise the phase. The sections below describe the methods used and examples of typical results for every phase.

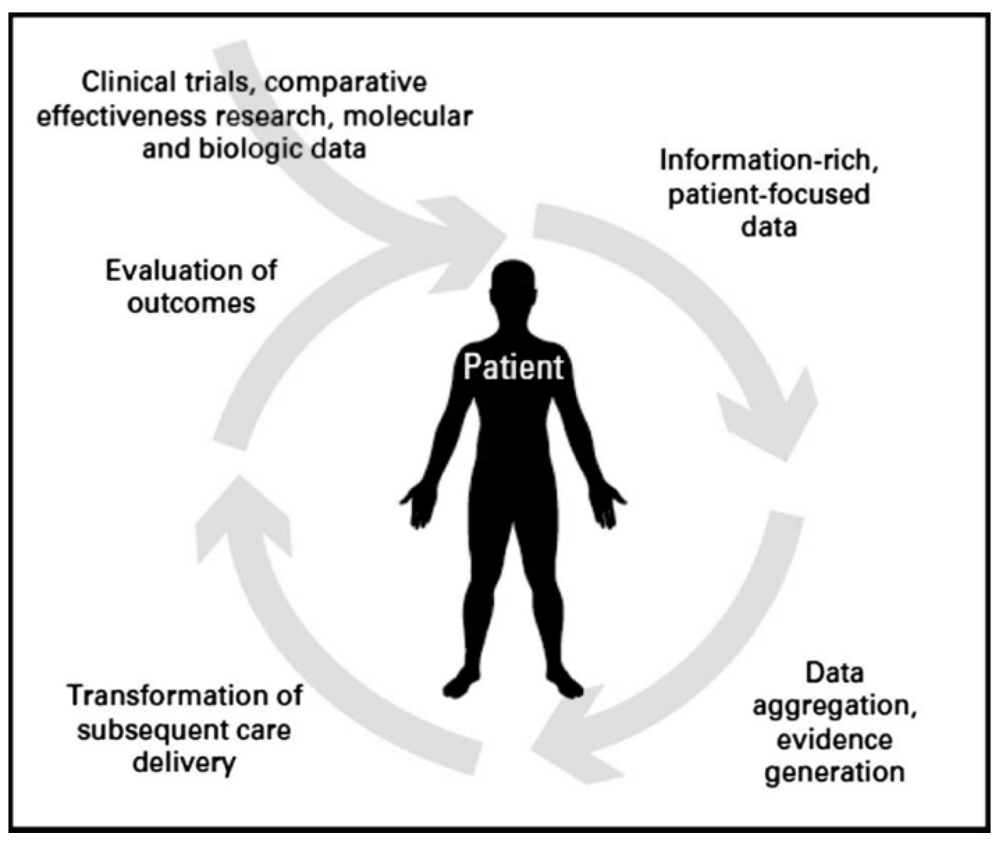

Figure 2 - Four phases of Rapid Learning [14].

Data

Rapid Learning requires both a great deal of data and a large diversity of data. The amount of data is important (a) to obtain higher quality knowledge (the quality of the knowledge correlates with the number of patients on which that knowledge is based) and (b) to be able to generate knowledge concerning smaller, more homogeneous patient groups and/or use more variables in the knowledge phase. The diversity of the data (particularly with respect to the treatments used, but also in terms of patient characteristics) is important to ultimately decide which treatment is best for an individual patient. Obtaining enough data of sufficient quality and diversity is the biggest challenge in Rapid Learning. This is only possible if data are shared across institutional and national borders, both academic and community health care systems. Such data sharing is hampered by a lack of time; differences in language and culture as well as data recording practices; the academic and political value of data; risks to reputation; privacy and legal aspects and so on. Nonetheless, one project that has made successful use of data sharing is euroCAT (www.eurocat.info), a collaborative project involving radiotherapy 
institutes in the Netherlands, Germany and Belgium. A crucial factor in the success of this project was the use of innovative information technologies, which made it possible to learn from each other's data without the data having to leave the institution (a concept known as distributed learning). Another important factor was the development of a dataset with semantic interoperability (also known as 'data with linguistic unity' or 'machine-readable data'), in which local terms are converted into concepts from a welldefined ontology (e.g., $\mathrm{NCl}$ Thesaurus, SNOMED). In such an approach, the ontology terms serve as a common interface to the data at each institutional site, enabling a common approach to information retrieval and reasoning facilitated through a semantic portal to the data. This semantic interoperability approach also allows one to add data from clinical trials to further strengthen the data available to Rapid Learning.

The data collected in routine clinical care are often of lower quality compared to data from clinical trials. Data captured in routine care are often incorrect, contradictory, missing and biased.

Although many problems are mitigated by the sheer volume of data, it is important to include data quality improvement protocols varying from simple logic (e.g. it is impossible to be $60 \mathrm{~kg}$ and have a BMI of 32) to more probabilistic approaches (e.g. for a similar patient cohort the median value of the maximal standard uptake value from ${ }^{18} \mathrm{~F}$ FDGDG PET scans should be similar between two institutes). A positive effect of such initiatives is that they give rise to increasing coordination with respect to what data need to be collected and how (i.e., disease-specific 'umbrella' protocols). The end users of the knowledge, the provider and the patient, not only need to gain insight into effects of various treatment options, but also in uncertainties, conflicting data, and toxicities and other treatment burden. It should be noted that getting data in the proposed manner does not mean that there is a need to capture more data, which would be an unacceptable additional burden to often overloaded professionals. Rather, the data that are already captured in routine care and in clinical trials are combined and re-used. There are various prototypes to do this such as in the euroCAT project where a fully automated, daily synchronisation of the clinical databases into a semantically interoperable dataset takes place.

\section{Knowledge}

Machine learning is used to extract knowledge from great amounts of data. In machine learning, models/algorithms are developed that best describe the data but that can also make predictions for new, unseen data. Models trained on retrospective data may be used to predict the outcomes (e.g., survival, quality of life, toxicity, etc.) of various treatments on the basis of data from a new patient. Obviously, it is crucial that such models are adequately validated [22]; a non-validated model is of very limited value. To this end, a validation set should always be available, preferably from a different institute than that from which the data were used to create the model. Examples of radiotherapy 
models (on the basis of both clinical trials and Rapid Learning) are available for nonsmall cell lung, rectal and head-and-neck cancer on http://www.predictcancer.org, breast cancer on http://research.nki.nl/ibr/ and glioblastoma on http://www.eortc.be/ tools/gbmcalculator/.

\section{Application}

In this phase, the knowledge generated by Rapid Learning is applied with the help of decision support systems (DSS). Typically, these are tools and software applications that can be used to apply knowledge-driven healthcare in practice. Examples include nomograms (as in Figure 3) [15, 16, 23-27] and websites such as those named above, for radiotherapy models, which help predict the expected treatment outcome of radiotherapy when they are supplied with the parameters specifically relevant to the clinical case. Decision support systems are neither intended nor suited as a replacement for the physician as a healthcare professional. They are designed to support the physician and the patient in making a more informed decision with respect to a particular treatment. The use of computer models to support healthcare professionals in their efforts is, of course, not new in radiation oncology. Physics-based computer models, with which doses can be better calculated than by hand, as well as radiobiology-based Normal Tissue Complications Probability (NTCP) and Tumour Control Probability (TCP) models to correlate the given dose with tumour control and toxicity, are commonplace within radiotherapy $[28,29]$. For example, geometrical models based on tumour volume alone have shown additional value next to classical TNM classification as well [30]. The new models emerging from Rapid Learning are a natural extension of this to patient outcomes. However, a key difference is that the Rapid Learning models are more 'holistic' and multifactorial than the current physics- or radiobiology-based models, as they also take patient, tumour and non-radiotherapy factors into account [31]. For instance, a Rapid Learning model of radiation-induced oesophagitis shows that the risk for this toxicity not only depends on the dose to which the oesophagus is exposed, but also greatly increases if chemotherapy is given concomitantly [32]. Another example is that the survival of non-metastatic unresectable non-small cell lung cancer is better predicted by a multifactorial model based on clinical and imaging variables, and even more when blood biomarkers are included $[32,33]$. In both cases the models outperform the prognostic value of TNM classification.

\section{Evaluation}

The underlying idea in Rapid Learning is that the application of knowledge acquired from routine data leads to predictability of treatment outcomes, meaning that these outcomes can be improved in terms of both effectiveness (achieving the desired result) and efficiency (the resources needed to achieve the result). Naturally, this needs to be 
continually evaluated, focusing on the question 'Is the outcome of the treatment as predicted?' Compared to the consensus- and evidence based guideline knowledge that is preferably constructed with (meta-analysis of) robust experimental data that are interpreted by multiple stakeholders including health care economists and patient representatives, the prediction models may suffer from confounders and election bias. For Rapid Learning, having high-quality data with respect to outcomes is crucial. This implies the use of broadly accepted taxonomies such as RECIST or pathological Complete Response for tumour response [34], CTCAE for toxicity [35] and euroQoL for quality of life \& utilities (which allow to calculate Quality Adjusted Life Year (QALY)) [36, 37]. Naturally, keeping thorough records of treatment outcomes is important not only for Rapid Learning, but also for initiatives such as the quality registration system for lung cancer patients initiated by the Dutch Society for Radiotherapy and Oncology.

\section{DISCUSSION}

Tailored cancer treatment is a necessity, to ensure not only that the individual patient receives the treatment that best suits his or her wishes, and to avoid under or overtreatment but also to optimize resources, so that the right resources are being used for the right patients in healthcare in a broader sense. However, tailored cancer treatment is also a challenge: the great diversity of cancer patients and treatments implies that it is by no means always clear which choice leads to which treatment outcome. Especially in cases where the treatment options under consideration have no clear clinical advantage in the outcome, a shared decision-making process can be employed in order to make the most of patient preferences. Tailored therapy is also necessary for radiotherapy. The radiosensitivity of tumours and normal tissues is often unknown, certainly not homogeneous within an individual patient, and even less so between patients [38-41]. In addition, the range of treatment options and thus the number of decisions that need to be made within radiotherapy have risen sharply, largely due to technological innovations such as IMRT, VMAT, IGRT and particle therapy as well as innovative combinations with systemic and targeted treatments such as tyrosine inhibitors or monoclonal antibodies (e.g., Cetuximab). Opting for a particular radiation treatment on the basis of expected outcomes is therefore difficult, and the established guidelines and literature provide only limited support in this regard.

This article has discussed Rapid Learning as a means of support when deciding on a tailored radiation treatment. In essence, Rapid Learning involves reusing local, clinical, routine data to develop knowledge in the form of models that can predict treatment outcomes, and then clinically applying and carefully evaluating these models by way of Decision Support Systems. The hypothesis is that treatment outcomes obtained in the past can be used to predict future results. 
Score

pT stage

cT

Age, years

pN stage

Concomitant chemotherapy

Adjuvant chemotherapy

Sum of scores

Probability of local

recurrence within 5 years, \%
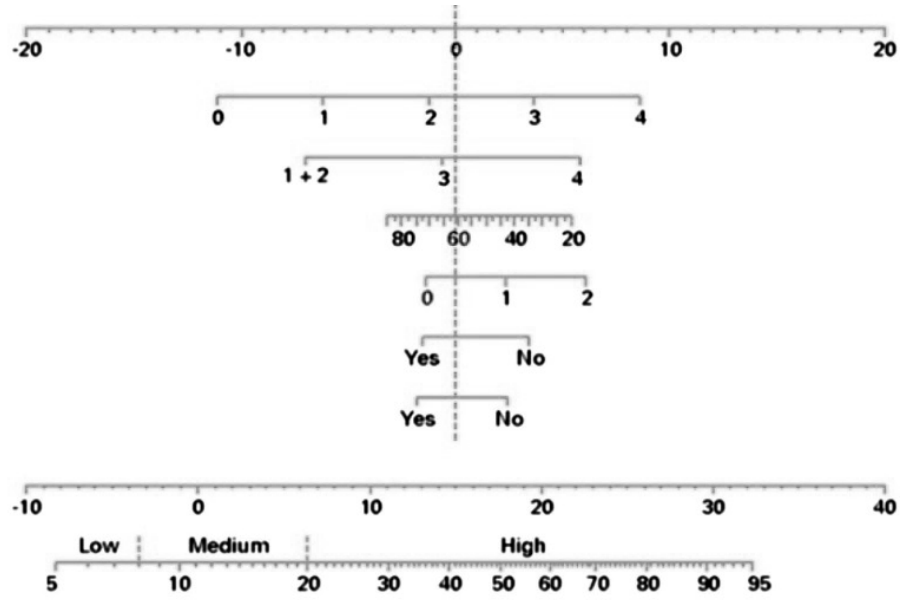

Figure 3 - Example of a nomogram [23].

Earlier attempts to introduce so-called 'expert systems' had mixed results. The proposed Rapid Learning methodology is different from the earlier attempts to deploy expert systems in several ways: it makes use of larger quantities of relevant data (e.g. the clinical patient population), as steadily more clinical data become available electronically in the clinical environment. This also enables validation in one's local practice which is a prerequisite for any expert system to be accepted, similar to commissioning and acceptance of treatment planning systems in radiotherapy. In contrast with expert systems, Rapid Learning employs quantitative models in addition to qualitative models. Finally, the de facto current expert system from "literature and guidelines based on clinical trials" has limited application to personalised medicine. This will drive the demand for more flexible and rapidly updated expert systems such as proposed in this review. The Rapid Learning approach seems to contradict the principles of evidence-based medicine, in which treatment decisions are based solely on results obtained from controlled clinical trials. In fact, it does not; both approaches are complementary (Figure 4). This is compounded by the fact that Rapid Learning is based on results obtained from the less controlled setting of clinical practice. These different environments yield different insights. Controlled clinical trials primarily aim to identify small improvements in results between two treatments in a patient group that is as homogeneous as possible. In contrast, Rapid Learning will reveal major differences in treatment outcomes that stem from the heterogeneity of the patient group. It will be inferior in detecting minor differences in treatments due to the lower quality of the data recorded in clinical practice as compared to the same treatment in a clinical trial. In addition, Rapid Learning can be seen as an alternative for situations in which there are insufficient evidence to make decisions in line with the principles of evidence-based medicine. This is often the case with technological innovations; for instance, when considering the use of new techniques (e.g., IMRT, 
protons) in the field of radiotherapy [42]. Rapid Learning is new and still needs to prove its value as a supplement to traditional, evidence-based approaches. There are several developments that might help Rapid Learning change the way scientific evidence is viewed in medicine: (a) Technological advances will be created by larger and higher quality databases that link electronic health records with research databases, as well as the advent of the Semantic Web with increased interoperability and distributed learning approaches that enable learning from data without the need for data to leave the hospital; (b) The development by domain experts of qualitative criteria to evaluate evidence coming from large databases and rapid learning approaches; (c) The increased pressure and possible reimbursement from healthcare payers to use Decision Support Systems, especially for high cost treatments such as proton therapy; and (d) The development of "clinical grade" and certified commercial decision support systems.

Radiotherapy seems to be the ideal setting to study the value of Rapid Learning, given the field's high degree of computerisation, as well as its long use and acceptance of predictive models. Within clinical radiotherapy, models and planning systems should become available that make it possible to not only plan on the basis of physical dose and Dose Volume Histograms parameters, but also to explain the relationship with the expected clinical outcomes in individual patients. Translating knowledge to an individual patient is challenging, particularly in so-called preference-sensitive situations where there are tradeoffs between options with more or less equally desirable outcomes, but in which different individuals may value differently e.g. in terms of side effects. As access to health-related information improves, patients have an increased desire to be in charge of their own life and health. Despite investment in efforts to improve the skills of clinicians, patients continue to report low levels of involvement [43]. There is indeed evidence level 1 from a Cochrane systematic review evaluating 86 studies involving 20,209 participants included in published randomized controlled trials demonstrating that decision aids increase people's involvement, support informed values-based choices in patient practitioner communication, and improve knowledge and realistic perception of outcomes. We therefore believe the next step will be to integrate, whenever possible, Shared Decision Making approaches (see for example www.treatmentchoice.info; www.optiongrid.org) to include the patient perspective in the choice of best treatment [27]. 


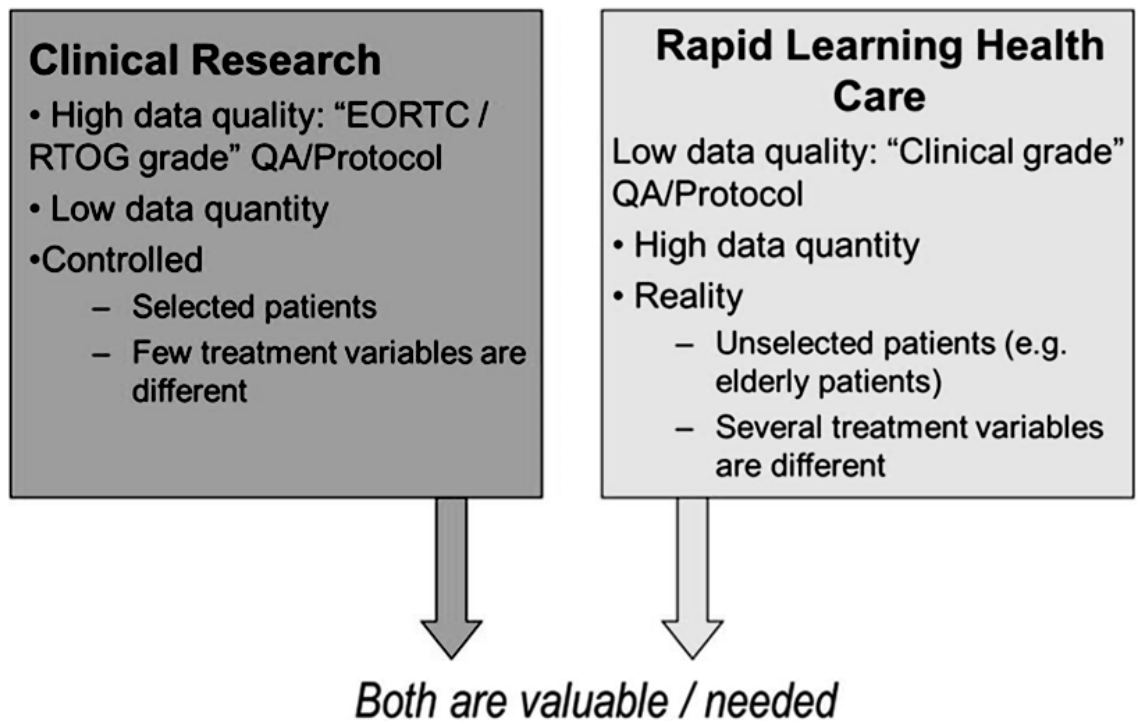

Figure 4-Complementary instead of contradictory approaches.

\section{CONFLICT OF INTEREST}

We are not aware of any actual or potential conflicts of interest.

\section{ACKNOWLEDGEMENTS}

We would like to acknowledge the financial support of the EU IMI programme (QuICConCePT), the CTMM framework (AIRFORCE project), the EU 6th and 7th framework programme (Metoxia, Artforce, Eureca), Interreg (www.eurocat.info) and the Dutch Cancer Society (KWF UM 2011-5020, KWF UM 2009-4454). 


\section{REFERENCES}

[1] Lambin P, Petit SF, Aerts HJ, van Elmpt WJ, Oberije CJ, Starmans MH, et al. The ESTRO Breur Lecture 2009. From population to voxel-based radiotherapy: exploiting intra-tumour and intra-organ heterogeneity for advanced treatment of non-small cell lung cancer. Radiotherapy and oncology : journal of the European Society for Therapeutic Radiology and Oncology. 2010 Aug;96(2):145-152.

[2] Koboldt DC, Fulton RS, McLellan MD. Comprehensive molecular portraits of human breast tumours. Nature. 2012 Oct 4;490(7418):61-70.

[3] Seigneuric R, Starmans MH, Fung G, Krishnapuram B, Nuyten DS, van Erk A, et al. Impact of supervised gene signatures of early hypoxia on patient survival. Radiotherapy and oncology : journal of the European Society for Therapeutic Radiology and Oncology. 2007 Jun;83(3):374-382.

[4] Starmans MH, Chu KC, Haider S, Nguyen F, Seigneuric R, Magagnin MG, et al. The prognostic value of temporal in vitro and in vivo derived hypoxia gene-expression signatures in breast cancer. Radiotherapy and oncology : journal of the European Society for Therapeutic Radiology and Oncology. 2012 Mar;102(3):436-443.

[5] Starmans MH, Fung G, Steck H, Wouters BG, Lambin P. A simple but highly effective approach to evaluate the prognostic performance of gene expression signatures. PloS one. 2011;6(12):e28320.

[6] Starmans MH, Lieuwes NG, Span PN, Haider S, Dubois L, Nguyen F, et al. Independent and functional validation of a multi-tumour-type proliferation signature. British journal of cancer. 2012 Jul 24;107(3):508-515.

[7] Starmans MH, Zips D, Wouters BG, Baumann M, Lambin P. The use of a comprehensive tumour xenograft dataset to validate gene signatures relevant for radiation response. Radiotherapy and oncology : journal of the European Society for Therapeutic Radiology and Oncology. 2009 Sep;92(3):417-422.

[8] Voets AM, Oberije C, Struijk RB, Reymen B, De Ruyck K, Thierens H, et al. No association between TGFbeta1 polymorphisms and radiation-induced lung toxicity in a European cohort of lung cancer patients. Radiotherapy and oncology : journal of the European Society for Therapeutic Radiology and Oncology. 2012 Dec;105(3):296-298.

[9] Movsas B, Moughan J, Owen J, Coia LR, Zelefsky MJ, Hanks G, et al. Who enrolls onto clinical oncology trials? A radiation Patterns Of Care Study analysis. International journal of radiation oncology, biology, physics. 2007 Jul 15;68(4):1145-1150.

[10] Grand MM, O'Brien PC. Obstacles to participation in randomised cancer clinical trials: a systematic review of the literature. Journal of medical imaging and radiation oncology. 2012 Feb;56(1):31-39.

[11] Murthy VH, Krumholz HM, Gross CP. Participation in cancer clinical trials: race-, sex-, and age-based disparities. Jama. 2004 Jun 9;291(22):2720-2726.

[12] Bastian H, Glasziou P, Chalmers I. Seventy-five trials and eleven systematic reviews a day: how will we ever keep up? PLoS medicine. 2010 Sep;7(9):e1000326.

[13] Deasy JO, Bentzen SM, Jackson A, Ten Haken RK, Yorke ED, Constine LS, et al. Improving normal tissue complication probability models: the need to adopt a "data-pooling" culture. International journal of radiation oncology, biology, physics. 2010 Mar 1;76(3 Suppl):S151-154.

[14] Abernethy AP, Etheredge LM, Ganz PA, Wallace P, German RR, Neti C, et al. Rapid-learning system for cancer care. Journal of clinical oncology : official journal of the American Society of Clinical Oncology. 2010 Sep 20;28(27):4268-4274.

[15] Dehing-Oberije C, Yu S, De Ruysscher D, Meersschout S, Van Beek K, Lievens Y, et al. Development and external validation of prognostic model for 2-year survival of non-small-cell lung cancer patients treated with chemoradiotherapy. International journal of radiation oncology, biology, physics. 2009 Jun $1 ; 74(2): 355-362$

[16] Egelmeer AG, Velazquez ER, de Jong JM, Oberije C, Geussens Y, Nuyts S, et al. Development and validation of a nomogram for prediction of survival and local control in laryngeal carcinoma patients treated with radiotherapy alone: a cohort study based on 994 patients. Radiotherapy and oncology : journal of the European Society for Therapeutic Radiology and Oncology. 2011 Jul;100(1):108-115. 
[17] Jimenez MF, van Baardwijk A, Aerts HJ, De Ruysscher D, Novoa NM, Varela G, et al. Effectiveness of surgery and individualized high-dose hyperfractionated accelerated radiotherapy on survival in clinical stage I non-small cell lung cancer. A propensity score matched analysis. Radiotherapy and oncology : journal of the European Society for Therapeutic Radiology and Oncology. 2010 Dec;97(3):413-417.

[18] Roelofs E, Engelsman M, Rasch C, Persoon L, Qamhiyeh S, de Ruysscher D, et al. Results of a multicentric in silico clinical trial (ROCOCO): comparing radiotherapy with photons and protons for non-small cell lung cancer. Journal of thoracic oncology : official publication of the International Association for the Study of Lung Cancer. 2012 Jan;7(1):165-176.

[19] Lambin P, Rios-Velazquez E, Leijenaar R, Carvalho S, van Stiphout RG, Granton P, et al. Radiomics: extracting more information from medical images using advanced feature analysis. European journal of cancer (Oxford, England : 1990). 2012 Mar;48(4):441-446.

[20] Roelofs E, Persoon L, Nijsten S, Wiessler W, Dekker A, Lambin P. Benefits of a clinical data warehouse with data mining tools to collect data for a radiotherapy trial. Radiotherapy and oncology : journal of the European Society for Therapeutic Radiology and Oncology. 2013 Jul;108(1):174-179.

[21] van Elmpt W, Nijsten S, Mijnheer B, Dekker A, Lambin P. The next step in patient-specific QA: 3D dose verification of conformal and intensity-modulated RT based on EPID dosimetry and Monte Carlo dose calculations. Radiotherapy and oncology : journal of the European Society for Therapeutic Radiology and Oncology. 2008 Jan;86(1):86-92.

[22] Steyerberg EW, Vickers AJ, Cook NR, Gerds T, Gonen M, Obuchowski N, et al. Assessing the performance of prediction models: a framework for traditional and novel measures. Epidemiology (Cambridge, Mass). 2010 Jan;21(1):128-138.

[23] Valentini V, van Stiphout RG, Lammering G, Gambacorta MA, Barba MC, Bebenek M, et al. Nomograms for predicting local recurrence, distant metastases, and overall survival for patients with locally advanced rectal cancer on the basis of European randomized clinical trials. Journal of clinical oncology : official journal of the American Society of Clinical Oncology. 2011 Aug 10;29(23):3163-3172.

[24] De Ruysscher D, Dehing C, Yu S, Wanders R, Ollers M, Dingemans AM, et al. Dyspnea evolution after high-dose radiotherapy in patients with non-small cell lung cancer. Radiotherapy and oncology : journal of the European Society for Therapeutic Radiology and Oncology. 2009 Jun;91(3):353-359.

[25] De Ruysscher D, Houben A, Aerts HJ, Dehing C, Wanders R, Ollers M, et al. Increased (18)F-deoxyglucose uptake in the lung during the first weeks of radiotherapy is correlated with subsequent RadiationInduced Lung Toxicity (RILT): a prospective pilot study. Radiotherapy and oncology : journal of the European Society for Therapeutic Radiology and Oncology. 2009 Jun;91(3):415-420.

[26] Dehing-Oberije C, De Ruysscher D, van Baardwijk A, Yu S, Rao B, Lambin P. The importance of patient characteristics for the prediction of radiation-induced lung toxicity. Radiotherapy and oncology : journal of the European Society for Therapeutic Radiology and Oncology. 2009 Jun;91(3):421-426.

[27] Stacey D, Bennett CL, Barry MJ, Col NF, Eden KB, Holmes-Rovner M, et al. Decision aids for people facing health treatment or screening decisions. The Cochrane database of systematic reviews. 2011(10):CD001431.

[28] Bentzen SM, Dorr W, Gahbauer R, Howell RW, Joiner MC, Jones B, et al. Bioeffect modeling and equieffective dose concepts in radiation oncology--terminology, quantities and units. Radiotherapy and oncology : journal of the European Society for Therapeutic Radiology and Oncology. 2012 Nov;105(2):266-268.

[29] De Leeuw AA, Van de Kamer JB, Moerland MA, Philippens ME, Jurgenliemk-Schulz IM. The effect of alternative biological modelling parameters (alpha/beta and half time of repair $T(1 / 2)$ ) on reported EQD2 values in the treatment of advanced cervical cancer. Radiotherapy and oncology : journal of the European Society for Therapeutic Radiology and Oncology. 2011 Nov;101(2):337-342.

[30] Guo R, Sun Y, Yu XL, Yin WJ, Li WF, Chen YY, et al. Is primary tumour volume still a prognostic factor in intensity modulated radiation therapy for nasopharyngeal carcinoma? Radiotherapy and oncology : journal of the European Society for Therapeutic Radiology and Oncology. 2012 Sep;104(3):294-299. 


\section{CHAPTER 2}

[31] Lambin P, van Stiphout RG, Starmans MH, Rios-Velazquez E, Nalbantov G, Aerts HJ, et al. Predicting outcomes in radiation oncology--multifactorial decision support systems. Nature reviews Clinical oncology. 2013 Jan;10(1):27-40.

[32] Dehing-Oberije C, De Ruysscher D, Petit S, Van Meerbeeck J, Vandecasteele K, De Neve W, et al. Development, external validation and clinical usefulness of a practical prediction model for radiationinduced dysphagia in lung cancer patients. Radiotherapy and oncology : journal of the European Society for Therapeutic Radiology and Oncology. 2010 Dec;97(3):455-461.

[33] Dehing-Oberije C, Aerts H, Yu S, De Ruysscher D, Menheere P, Hilvo M, et al. Development and validation of a prognostic model using blood biomarker information for prediction of survival of non-small-cell lung cancer patients treated with combined chemotherapy and radiation or radiotherapy alone (NCT00181519, NCT00573040, and NCT00572325). International journal of radiation oncology, biology, physics. 2011 Oct 1;81(2):360-368.

[34] Eisenhauer EA, Therasse P, Bogaerts J, Schwartz LH, Sargent D, Ford R, et al. New response evaluation criteria in solid tumours: revised RECIST guideline (version 1.1). European journal of cancer (Oxford, England : 1990). 2009 Jan;45(2):228-247.

[35] Trotti A, Colevas AD, Setser A, Rusch V, Jaques D, Budach V, et al. CTCAE V3.0: development of a comprehensive grading system for the adverse effects of cancer treatment. Seminars in radiation oncology. 2003 Jul;13(3):176-181.

[36] Grutters JP, Kessels AG, Pijls-Johannesma M, De Ruysscher D, Joore MA, Lambin P. Comparison of the effectiveness of radiotherapy with photons, protons and carbon-ions for non-small cell lung cancer: a meta-analysis. Radiotherapy and oncology : journal of the European Society for Therapeutic Radiology and Oncology. 2010 Apr;95(1):32-40.

[37] Peeters A, Grutters JP, Pijls-Johannesma M, Reimoser S, De Ruysscher D, Severens JL, et al. How costly is particle therapy? Cost analysis of external beam radiotherapy with carbon-ions, protons and photons. Radiotherapy and oncology : journal of the European Society for Therapeutic Radiology and Oncology. 2010 Apr;95(1):45-53.

[38] Aerts HJ, Bussink J, Oyen WJ, van Elmpt W, Folgering AM, Emans D, et al. Identification of residual metabolic-active areas within NSCLC tumours using a pre-radiotherapy FDG-PET-CT scan: a prospective validation. Lung cancer (Amsterdam, Netherlands). 2012 Jan;75(1):73-76.

[39] van Loon J, Janssen MH, Ollers M, Aerts HJ, Dubois L, Hochstenbag M, et al. PET imaging of hypoxia using [18F]HX4: a phase I trial. European journal of nuclear medicine and molecular imaging. 2010 Aug;37(9):1663-1668.

[40] Mortensen LS, Johansen J, Kallehauge J, Primdahl H, Busk M, Lassen P, et al. FAZA PET/CT hypoxia imaging in patients with squamous cell carcinoma of the head and neck treated with radiotherapy: results from the DAHANCA 24 trial. Radiotherapy and oncology : journal of the European Society for Therapeutic Radiology and Oncology. 2012 Oct;105(1):14-20.

[41] Zips D, Zophel K, Abolmaali N, Perrin R, Abramyuk A, Haase R, et al. Exploratory prospective trial of hypoxia-specific PET imaging during radiochemotherapy in patients with locally advanced head-and-neck cancer. Radiotherapy and oncology : journal of the European Society for Therapeutic Radiology and Oncology. 2012 Oct;105(1):21-28.

[42] Langendijk JA, Lambin P, De Ruysscher D, Widder J, Bos M, Verheij M. Selection of patients for radiotherapy with protons aiming at reduction of side effects: the model-based approach. Radiotherapy and oncology : journal of the European Society for Therapeutic Radiology and Oncology. 2013 Jun;107(3):267-273.

[43] Stiggelbout AM, Van der Weijden T, De Wit MP, Frosch D, Legare F, Montori VM, et al. Shared decision making: really putting patients at the centre of healthcare. BMJ (Clinical research ed). 2012;344:e256. 


\section{Chapter}

\section{Radiomics: Extracting more information from medical images using advanced feature analysis}

Published in: European Journal of Cancer, 48(4), 2012 Mar: 441-446

Radiomics: Extracting more information from medical images using advanced feature analysis

Philippe Lambin, Emmanuel Rios-Velazquez, Ralph Leijenaar, Sara Carvalho, Ruud G.P.M. van Stiphout, Patrick Granton, Catharina M.L. Zegers, Robert Gillies, Ronald Boellard, André Dekker, and Hugo J.W.L. Aerts 


\section{ABSTRACT}

Solid cancers are spatially and temporally heterogeneous. This limits the use of invasive biopsy based molecular assays but gives huge potential for medical imaging, which has the ability to capture intra-tumoural heterogeneity in a non-invasive way. During the past decades, medical imaging innovations with new hardware, new imaging agents and standardised protocols, allows the field to move towards quantitative imaging. Therefore, also the development of automated and reproducible analysis methodologies to extract more information from image-based features is a requirement. Radiomics - the high-throughput extraction of large amounts of image features from radiographic images - addresses this problem and is one of the approaches that hold great promises but need further validation in multi-centric settings and in the laboratory. 


\section{INTRODUCTION}

The use and role of medical imaging technologies in clinical oncology has greatly expanded from primarily a diagnostic tool to include a more central role in the context of individualised medicine over the past decade (Figure 1). It is expected that imaging contains complementary and interchangeable information compared to other sources, e.g. demographics, pathology, blood biomarkers, genomics and that combining these sources of information will improve individualised treatment selection and monitoring [1].

Cancer can be probed in many ways depending on the non-invasive imaging device used or the mode by which it operates (Figure 2). Classically, anatomical computed tomography (CT) imaging is an often used modality, acquiring images of the 'anatome' in high resolution (e.g. $1 \mathrm{~mm}^{3}$ ). CT imaging is now routinely used and is playing an essential role in all phases of cancer management, including prediction, screening, biopsy guidance for detection, treatment planning, treatment guidance and treatment response evaluation $[2,3]$. CT is used in the assessment of structural features of cancer but it is not perceived to portray functional or molecular details of solid tumours. Functional imaging concerns physiological processes and functions such as diffusion, perfusion and glucose uptake. Here, commonly used methodologies are dynamic contract enhanced-magnetic resonance imaging (DCE-MRI), assessing tumour perfusion and fluoro-2-Deoxy-D-glucose (FDG) positron emission tomography (PET) imaging, assessing tumour metabolism, which both often are found to have prognostic value [4-6]. Finally, another modality is molecular imaging, visualising at the level of specific pathways or macro-molecule in vivo. For example, there are molecular markers assessing tumour hypoxia or labelled antibodies, assessing receptor expression levels of a tumour [1, 7].

Over the past decades, medical imaging has progressed in four distinct ways:

- Innovations in medical devices (hardware): This concerns improvements in imaging hardware and the development of combined modality machines. For example, in the last decade we moved from single slice CT to multiple slices CT and CT/PET. More recent developments are dual-source and dual-energy CT. These techniques significantly increase the temporal resolution for 4-D CT reconstructions allowing visualisation of fine structures in tissues, also in several stages in the cardiac or respiration phase. Moreover, dual-energy CT can be used to improve identification of tissue composition and density.

- Innovations in imaging agents: Innovations in imaging agents (or imaging biomarker, imaging probe, radiotracer), i.e. molecular substances injected in the body and used as an indicator of a specific biological process occurring in the body. This is achieved by contrast agents, i.e. an imaging agent using positive emission tomography (radiotracer). 
- Standardised protocol allowing quantitative imaging: Historically radiology has been a qualitative science, perhaps with the exception of the quantitative use of CT based electron densities in radiotherapy treatment planning. The use of standardised protocols like common MRI spin-echo sequences helps to allow multicentric use of imaging as well as transforming radiology to a more quantitative, highly reproducible science.
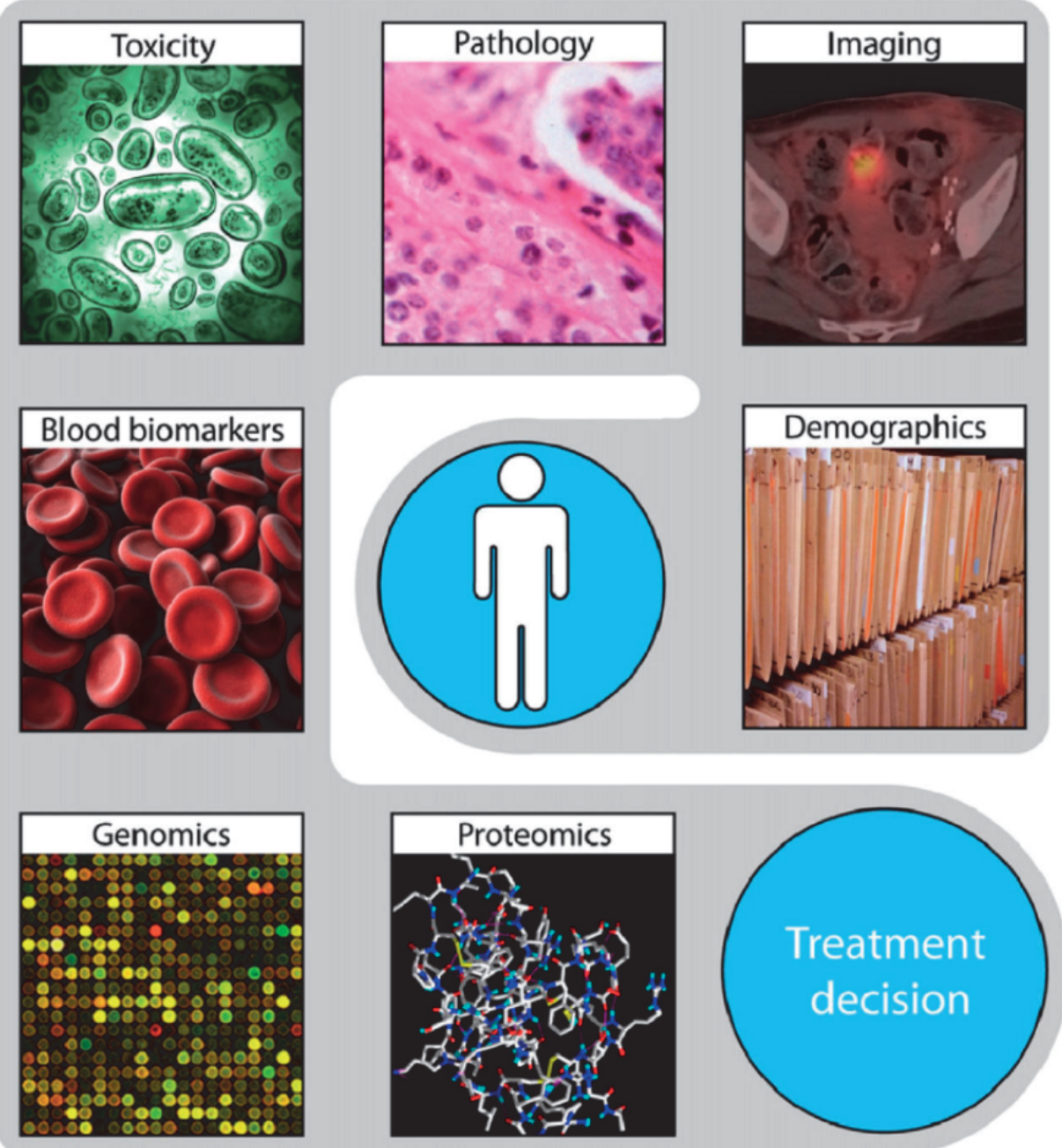

Figure 1 - Different sources of information, e.g. demographics, imaging, pathology, toxicity, biomarkers, genomics and proteomics, can be used for selecting the optimal treatment.

- Innovations in imaging analysis: The analysis of medical images has a large impact on the conclusions of the derived images. More and more software is becoming 
available, allowing for more quantification and standardisation. This has been illustrated by the development of the computer-assisted detection (CAD systems) that improves the performance of detecting cancer in mammography or in lung cancer diseases [8].

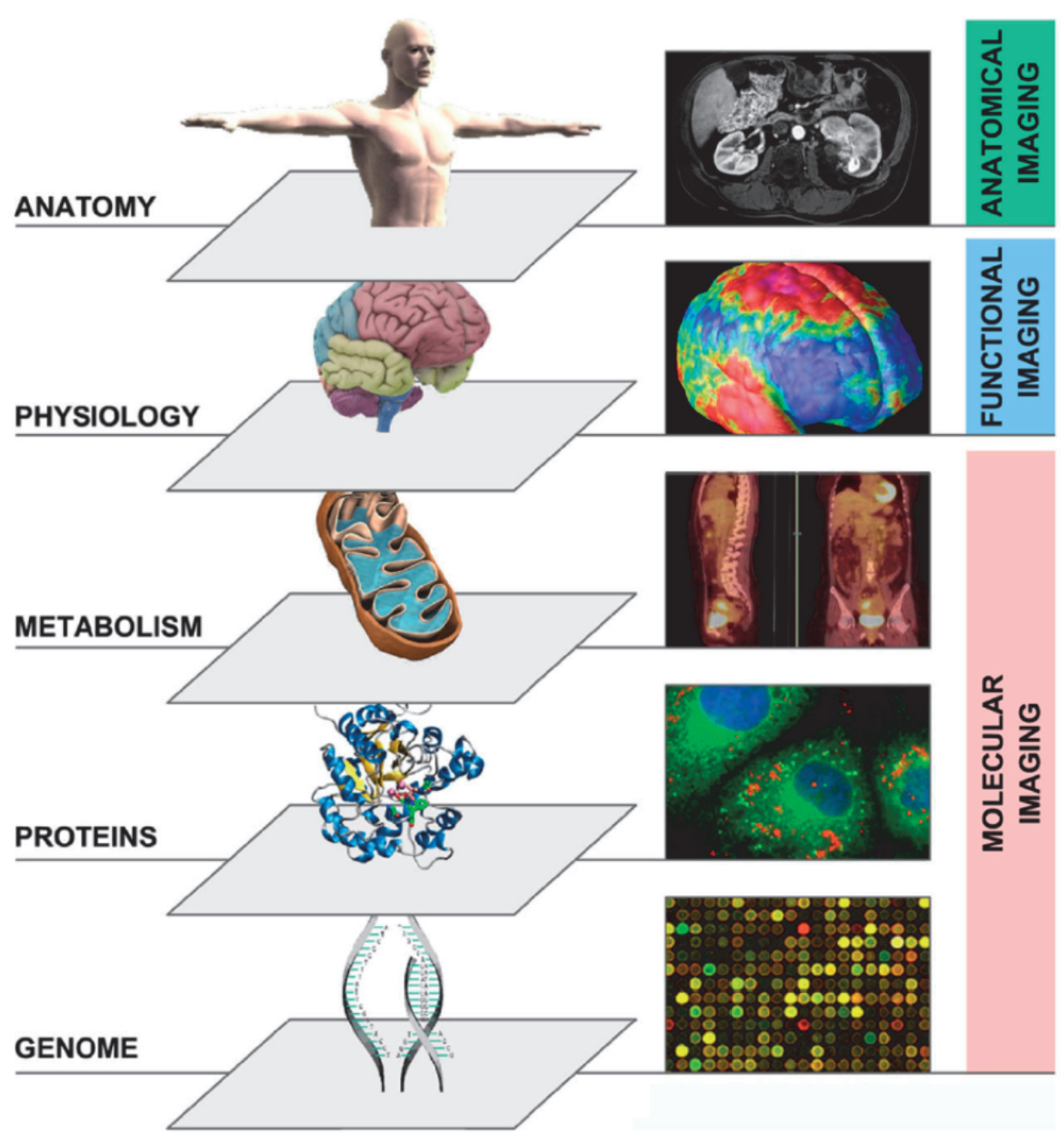

Figure 2 - Multilevel imaging: anatomical, functional, and molecular imaging.

A common use is to find indications of pathological processes, e.g. hypoxia markers using PET imaging.

Radiomics focuses on improvements of image analysis, using an automated highthroughput extraction of large amounts (200+) of quantitative features of medical images and belongs to the last category of innovations in medical imaging analysis. The 
hypothesis is that quantitative analysis of medical image data through automatic or semi-automatic software of a given imaging modality can provide more and better information than that of a physician. This is supported by the fact that patients exhibit differences in tumour shape and texture measurable by different imaging modalities (Figure 3).

\section{THE WORKFLOW OF RADIOMICS: A (SEMI) HIGH-THROUGHPUT APPROACH}

Figure 4 depicts the processes involved in the Radiomics workflow. The first step involves the acquisition of high quality and standardised imaging, for diagnostic or planning purposes. From this image, the macroscopic tumour is defined, either with an automated segmentation method or alternatively by an experienced radiologist or radiation oncologist. Quantitative imaging features are subsequently extracted from the previously defined tumour region. These features involve descriptors of intensity distribution, spatial relationships between the various intensity levels, texture heterogeneity patterns, descriptors of shape and of the relations of the tumour with the surrounding tissues (i.e. attachment to the pleural wall in lung, differentiation). The extracted image traits are then subjected to a feature selection procedure. The most informative features are identified based on their independence from other traits, reproducibility and prominence on the data. The selected features are then analysed for their relationship with treatment outcomes or gene expression. The ultimate goal is to provide accurate risk stratification by incorporating the imaging traits into predictive models for treatment outcome and to evaluate their added value to commonly used predictors.

\section{THE RADIOMICS HYPOTHESIS: INFERRING PROTEO-GENOMIC AND PHENOTYPIC INFORMATION FROM RADIOLOGICAL IMAGES}

The underlying hypothesis of Radiomics is that advanced image analysis on conventional and novel medical imaging could capture additional information not currently used, and more specifically, that genomic and proteomics patterns can be expressed in terms of macroscopic image-based features. If proven, we can infer phenotypes or gene-protein signatures, possibly containing prognostic information, from the quantitative analysis of medical image data. 
A

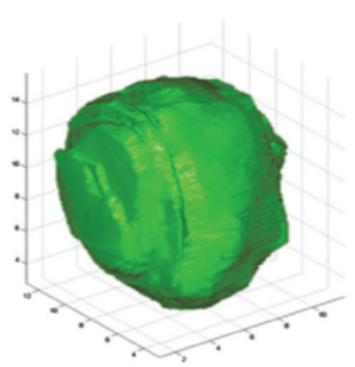

B
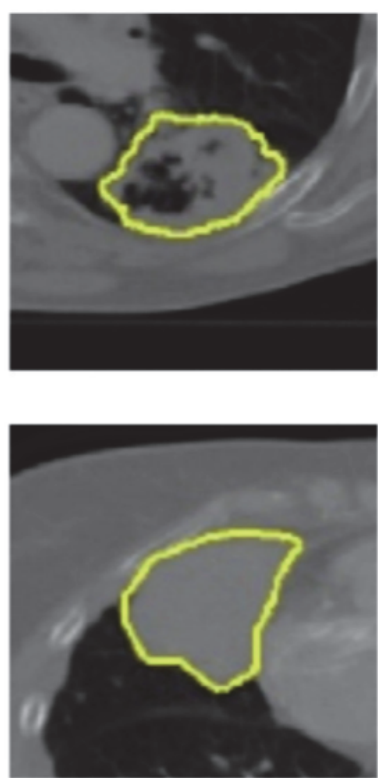

C
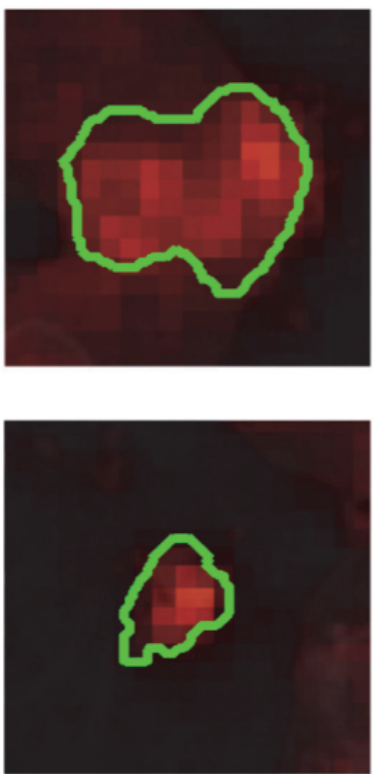

Figure 3 - (A) Two representative 3-D representations of a round tumour (top) and spiky tumour (bottom) measured by computed tomography (CT) imaging. (B) Texture differences between non-small cell lung cancer (NSCLC) tumours measured using CT imaging, more heterogeneous (top) and more homogeneous (bottom).

(C) Differences of FDG-PET uptake, showing heterogeneous uptake.

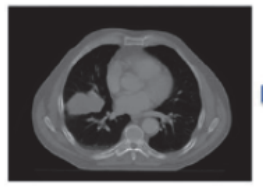

Imaging

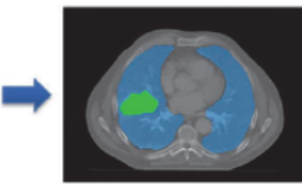

Segmentation

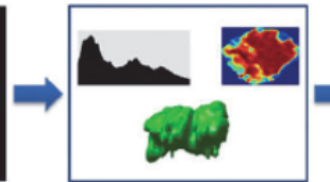

Feature extraction

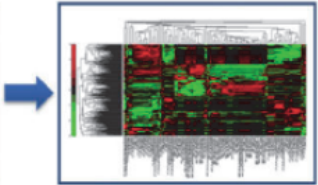

Analysis

Figure 4 - The Radiomics workflow. On the medical images, segmentation is performed to define the tumour region. From this region the features are extracted, e.g. features based on tumour intensity, texture and shape. Finally, these features are used for analysis, e.g. the features are assessed for their prognostic power, or linked with stage, or gene expression.

This hypothesis is supported by image-guided biopsies, which demonstrated that tumours show spatial differences in protein expressions [9]. More specifically, it has been demonstrated that major differences in protein expression patterns within a tumour can be correlated to radiographic findings (or radiophenotypes) such as contrastenhanced and non-enhanced regions based on CT data [10]. The authors suggest that image-guided proteomics holds promise for characterising tissues prior to treatment decisions and without imaging there is indeed a risk that the optimum treatment decision could be neglected (i.e. the use or not of a targeted agent). Also, Kuo et al. report- 
ed the association of CT-derived imaging traits with histo-pathologic markers, and several pre-defined gene expression modules on liver cancer [11, 12]. In ovarian carcinoma, an imaging feature describing the enhancement fraction as proportion of enhancing tumour tissue on a pre-treatment CT scan, was found predictive for outcome after first line chemotherapy [13]. In lung cancer, CT derived information has been limited to pre-treatment assessment of tumour volume and as response evaluation defined as tumour size reduction [14]. For PET imaging, the maximum and median FDG uptake has often been investigated, indicating strong prognostic power $[6,15]$. However, more complex descriptions of FDG uptake are only investigated on a limited scale. There was a study of El Naqa et al. [16], investigating the predictive power of intensity-volume histogram (IVH) metrics, shape and texture features to assess response to treatment of a limited set of patients with head and neck and cervix cancers. Tixier et al. also explored the potential of SUV based, shape and texture features extracted from baseline FDG-PET, images, to assess response to therapy and prognosis in order to predict response to combined chemo-radiation treatment in oesophageal cancer [17]. Also, textural features in FDG PET images exhibited small variations due to different acquisition modes and reconstruction parameters [18]. These examples open the question of whether quantitative extraction of additional imaging features on conventional imaging improves the ability of currently used parameters to predict or monitor response to treatment.

Furthermore, Radiomics can be linked with the concept of radio-genomics, which assumes that imaging features are related to gene signatures. An interesting finding in recent literature is that tumours with more genomic heterogeneity are more likely to develop a resistance to treatment and to metastasise [19]. This links to the concept that more heterogeneous tumours have a worse prognosis. According to the Radiomics hypothesis, the genomic heterogeneity could translate to an expression in an intratumoural heterogeneity that could be assessed through imaging and that would ultimately exhibit worse prognosis. This hypothesis has been sustained by Jackson et al. [15] and as well as by Diehn et al. [20] who quite convincingly showed that proliferation and hypoxia gene expression patterns can be predicted by mass effect and tumour contrast enhancement, respectively. They also showed that a specific imaging pattern could predict overexpression of epidermal growth factor receptor (EGFR), a known therapeutic target. Moreover, in their analysis the presence of certain image features was highly predictive of outcome. The authors concluded that imaging in this case MR provided an 'in vivo portrait' of genome-wide gene expression in glioblastoma multiform. Similar findings have been found in hepatocellular carcinomas by Segal et al. [21], showing that the combination of only 28 imaging traits was sufficient to reconstruct the variation of 116 gene expression modules.

These types of studies will need to be extended, by including more patients with external validation datasets, more tumour types that exhibit phenotypes such as invasiveness. This will be the focus of the QuIC-ConCePT consortium, to confirm experimen- 
tally the Radiomics hypothesis, namely to establish a causal relationship between gene expression patterns and image features.

\section{CONCLUSIONS}

Solid cancers have extraordinarily spatial and temporal heterogeneity at different levels: genes, proteins, cells, microenvironment, tissues and organs. This limits the use of biopsy based molecular assays but in contrast gives a huge potential for non-invasive imaging, which has the ability to capture intra-tumoural heterogeneity in a non-invasive way. Medical imaging innovations with new hardware, new imaging agents and standardised protocol now allow for quantitative imaging but require the development of 'smart' automated software to extract more information from image-based features. Radiomics - the high-throughput extraction of image features from radiographic images - is one approach that holds great promises but needs further validation in a multi-centric setting and in the laboratory.

\section{ACKNOWLEDGMENTS}

The authors are members of the QuIC-ConCePT project partly funded by EFPIA companies and the Innovative Medicine Initiative Joint Undertaking (IMI JU) under Grant Agreement No. 115151. We also acknowledge financial support from the CTMM framework (AIRFORCE project), EU 6th and 7th framework program (Euroxy and Metoxia program), Interreg (www.eurocat.info), and the Dutch Cancer Society (KWF UM 20115020, KWF UM 2009-4454). 


\section{REFERENCES}

[1] Lambin P, Petit SF, Aerts HJ, van Elmpt WJ, Oberije CJ, Starmans MH, et al. The ESTRO Breur Lecture 2009. From population to voxel-based radiotherapy: exploiting intra-tumour and intra-organ heterogeneity for advanced treatment of non-small cell lung cancer. Radiotherapy and oncology : journal of the European Society for Therapeutic Radiology and Oncology. 2010 Aug;96(2):145-152.

[2] Fass L. Imaging and cancer: a review. Molecular oncology. 2008 Aug;2(2):115-152.

[3] Eisenhauer EA, Therasse P, Bogaerts J, Schwartz LH, Sargent D, Ford R, et al. New response evaluation criteria in solid tumours: revised RECIST guideline (version 1.1). European journal of cancer (Oxford, England : 1990). 2009 Jan;45(2):228-247.

[4] van Baardwijk A, Dooms C, van Suylen RJ, Verbeken E, Hochstenbag M, Dehing-Oberije C, et al. The maximum uptake of (18)F-deoxyglucose on positron emission tomography scan correlates with survival, hypoxia inducible factor-1alpha and GLUT-1 in non-small cell lung cancer. European journal of cancer (Oxford, England : 1990). 2007 Jun;43(9):1392-1398.

[5] Berghmans T, Dusart M, Paesmans M, Hossein-Foucher C, Buvat I, Castaigne C, et al. Primary tumour standardized uptake value (SUVmax) measured on fluorodeoxyglucose positron emission tomography (FDG-PET) is of prognostic value for survival in non-small cell lung cancer (NSCLC): a systematic review and meta-analysis (MA) by the European Lung Cancer Working Party for the IASLC Lung Cancer Staging Project. Journal of thoracic oncology : official publication of the International Association for the Study of Lung Cancer. 2008 Jan;3(1):6-12.

[6] Paesmans M, Berghmans T, Dusart M, Garcia C, Hossein-Foucher C, Lafitte JJ, et al. Primary tumour standardized uptake value measured on fluorodeoxyglucose positron emission tomography is of prognostic value for survival in non-small cell lung cancer: update of a systematic review and metaanalysis by the European Lung Cancer Working Party for the International Association for the Study of Lung Cancer Staging Project. Journal of thoracic oncology : official publication of the International Association for the Study of Lung Cancer. 2010 May;5(5):612-619.

[7] Aerts HJ, Dubois L, Perk L, Vermaelen P, van Dongen GA, Wouters BG, et al. Disparity between in vivo EGFR expression and 89Zr-labeled cetuximab uptake assessed with PET. Journal of nuclear medicine : official publication, Society of Nuclear Medicine. 2009 Jan;50(1):123-131.

[8] Li H, Giger ML, Yuan Y, Chen W, Horsch K, Lan L, et al. Evaluation of computer-aided diagnosis on a large clinical full-field digital mammographic dataset. Academic radiology. 2008 Nov;15(11):1437-1445.

[9] Van Meter T, Dumur C, Hafez N, Garrett C, Fillmore H, Broaddus WC. Microarray analysis of MRI-defined tissue samples in glioblastoma reveals differences in regional expression of therapeutic targets. Diagnostic molecular pathology : the American journal of surgical pathology, part B. 2006 Dec;15(4):195-205.

[10] Hobbs SK, Shi G, Homer R, Harsh G, Atlas SW, Bednarski MD. Magnetic resonance image-guided proteomics of human glioblastoma multiforme. Journal of magnetic resonance imaging : JMRI. 2003 Nov; 18(5):530-536.

[11] Kuo MD, Gollub J, Sirlin CB, Ooi C, Chen X. Radiogenomic analysis to identify imaging phenotypes associated with drug response gene expression programs in hepatocellular carcinoma. Journal of vascular and interventional radiology : JVIR. 2007 Jul;18(7):821-831.

[12] Rutman AM, Kuo MD. Radiogenomics: creating a link between molecular diagnostics and diagnostic imaging. European journal of radiology. 2009 May;70(2):232-241.

[13] O'Connor JP, Jayson GC, Jackson A, Ghiorghiu D, Carrington BM, Rose CJ, et al. Enhancing fraction predicts clinical outcome following first-line chemotherapy in patients with epithelial ovarian carcinoma. Clinical cancer research : an official journal of the American Association for Cancer Research. 2007 Oct 15;13(20):6130-6135.

[14] Dehing-Oberije C, Aerts H, Yu S, De Ruysscher D, Menheere P, Hilvo M, et al. Development and validation of a prognostic model using blood biomarker information for prediction of survival of non-small-cell lung cancer patients treated with combined chemotherapy and radiation or radiotherapy alone 
(NCT00181519, NCT00573040, and NCT00572325). International journal of radiation oncology, biology, physics. 2011 Oct 1;81(2):360-368.

[15] Jackson A, O'Connor JP, Parker GJ, Jayson GC. Imaging tumour vascular heterogeneity and angiogenesis using dynamic contrast-enhanced magnetic resonance imaging. Clinical cancer research : an official journal of the American Association for Cancer Research. 2007 Jun 15;13(12):3449-3459.

[16] El Naqa I, Grigsby P, Apte A, Kidd E, Donnelly E, Khullar D, et al. Exploring feature-based approaches in PET images for predicting cancer treatment outcomes. Pattern recognition. 2009 Jun 1;42(6):1162-1171.

[17] Tixier F, Le Rest CC, Hatt M, Albarghach N, Pradier O, Metges JP, et al. Intratumour heterogeneity characterized by textural features on baseline 18F-FDG PET images predicts response to concomitant radiochemotherapy in esophageal cancer. Journal of nuclear medicine : official publication, Society of Nuclear Medicine. 2011 Mar;52(3):369-378.

[18] Galavis PE, Hollensen C, Jallow N, Paliwal B, Jeraj R. Variability of textural features in FDG PET images due to different acquisition modes and reconstruction parameters. Acta oncologica (Stockholm, Sweden). 2010 Oct;49(7):1012-1016.

[19] Campbell PJ, Yachida S, Mudie L, Stephens PJ, Pleasance ED, Stebbings LA, et al. The patterns and dynamics of genomic instability in metastatic pancreatic cancer. Nature. 2010 Oct 28;467(7319):1109-1113.

[20] Diehn M, Nardini C, Wang DS, McGovern S, Jayaraman M, Liang Y, et al. Identification of noninvasive imaging surrogates for brain tumour gene-expression modules. Proceedings of the National Academy of Sciences of the United States of America. 2008 Apr 1;105(13):5213-5218.

[21] Segal E, Sirlin CB, Ooi C, Adler AS, Gollub J, Chen X, et al. Decoding global gene expression programs in liver cancer by noninvasive imaging. Nature biotechnology. 2007 Jun;25(6):675-680. 



\section{Chapter

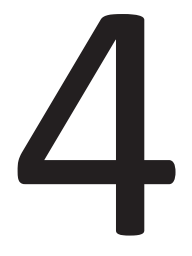

\section{Prognostic value of metabolic metrics extracted from baseline positron emission tomography images in non-small cell lung cancer}

Published in: Acta Oncologica, 52(7), 2013 Oct: 1398-404

Prognostic value of metabolic metrics extracted from baseline positron emission tomography images in non-small cell lung cancer

Sara Carvalho*, Ralph T. H. Leijenaar*, Emmanuel Rios Velazquez, Cary Oberije, Chintan Parmar, Wouter van Elmpt, Bart Reymen, Esther G. C. Troost, Michel Oellers, Andre Dekker, Robert Gillies, Hugo J. W. L. Aerts, Philippe Lambin

* These authors contributed equally to this work 


\begin{abstract}
introduction

Maximum, mean and peak SUV of primary tumour at baseline FDG-PET scans, have often been found predictive for overall survival in non-small cell lung cancer (NSCLC) patients. In this study we further investigated the prognostic power of advanced metabolic metrics derived from intensity volume histograms (IVH) extracted from PET imaging.
\end{abstract}

\title{
Methods
}

A cohort of 220 NSCLC patients (mean age, 66.6 years; 149 men, 71 women), stages I IIIB, treated with radiotherapy with curative intent were included (NCT00522639). Each patient underwent standardized pre-treatment CT-PET imaging. Primary GTV was delineated by an experienced radiation oncologist on CT-PET images. Common PET descriptors such as maximum, mean and peak SUV, and metabolic tumour volume (MTV) were quantified. Advanced descriptors of metabolic activity were quantified by IVH. These comprised five groups of features: absolute and relative volume above relative intensity threshold (AVRI and RVRI), absolute and relative volume above absolute intensity threshold (AVAI and RVAI), and absolute intensity above relative volume threshold (AIRV). MTV was derived from the IVH curves for volumes with SUV above 2.5, 3 and 4, and of $40 \%$ and $50 \%$ maximum SUV. Univariable analysis using Cox Proportional Hazard Regression was performed for overall survival (OS) assessment.

\section{Results}

Relative volume above higher SUV (80\%) was an independent predictor of OS ( $p=0.05)$. None of the possible surrogates for MTV based on volumes above SUV of 3,40\% and $50 \%$ of maximum SUV showed significant associations with OS [p $\left(\mathrm{AVAl}_{3}\right)=0.10$, $\left.p\left(A \vee A I_{4}\right)=0.22, p\left(A \vee R I_{40 \%}\right)=0.15, p\left(A \vee R I_{50 \%}\right)=0.17\right]$. Maximum and peak SUV $(r=0.99)$ revealed no prognostic value for OS $[p$ (maximum SUV) $=0.20, p($ peak SUV $)=0.22]$.

\section{Conclusions}

New methods using more advanced imaging features extracted from PET were analysed. Best prognostic value for OS of NSCLC patients was found for relative portions of the tumour above higher uptakes ( $80 \%$ SUV). 


\section{INTRODUCTION}

Lung cancer is the most common cancer type worldwide, accounting for more than 1.1 million deaths annually. Out of the total number of cases, $85 \%$ are of the non-small cell lung cancer (NSCLC) type. The 5-year survival for stages I-II and III is disappointingly low at respectively $50 \%$ and $20 \%$, mainly due to a high rate of loco-regional and distant progression [1].

Nowadays, much research largely focuses on prediction of treatment outcome based on patient and tumour characteristics prior to treatment. This would allow individualizing treatment and enhancing therapeutic approaches [2]. Here, imaging can play a crucial role as it allows for a non-invasive identification of the tumour [3]. Indeed, functional information gathered by Positron Emission Tomography (PET) has already been shown to be an effective tool in detecting early tumour alterations before any anatomical change is noticeable and detectable [4]. However, the possibility to predict response to treatment or overall survival based solely on pre-treatment scans is still under investigation and so far only a few metrics based on PET imaging have been explored to this end.

Currently, ${ }^{18}$ F-fluoro-2-Deoxy-D-glucose (FDG) is the most commonly used PET tracer in oncology. The investigation of prediction of tumour response based on PET features is mainly limited to simple measurements of Standard Uptake Value (SUV) such as maximum, mean and peak (defined within the neighbourhood of maximum SUV) [5]. There is still limited confirmation whether these different SUV descriptors in pretreatment scans are predictive [6].

Previous research also describes Metabolic Tumour Volume (MTV) and the Total Lesion Glycolysis (TLG) calculated for tumour volumes derived from semi-automatic segmentation methods based on PET imaging. MTV is defined for absolute SUV thresholds of lower value or relative uptakes in regard to the maximum SUV [7]. TLG is then expressed as the product of MTV by its mean SUV [7-9].

We investigated the prognostic value of metrics based on the metabolic information of PET imaging. These metrics are based on Intensity Volume Histograms (IVH), which summarize in a single curve the relation of tumour volume and SUV intensity. We hypothesized that the metabolic information described by these IVH curves could be used to predict treatment outcome of NSCLC patients.

The aim of this study was to assess the prognostic value of these IVH metrics for NSCLC patients treated with (chemo)radiotherapy and compare the results against the performance of the commonly used metrics based on SUV descriptors. 

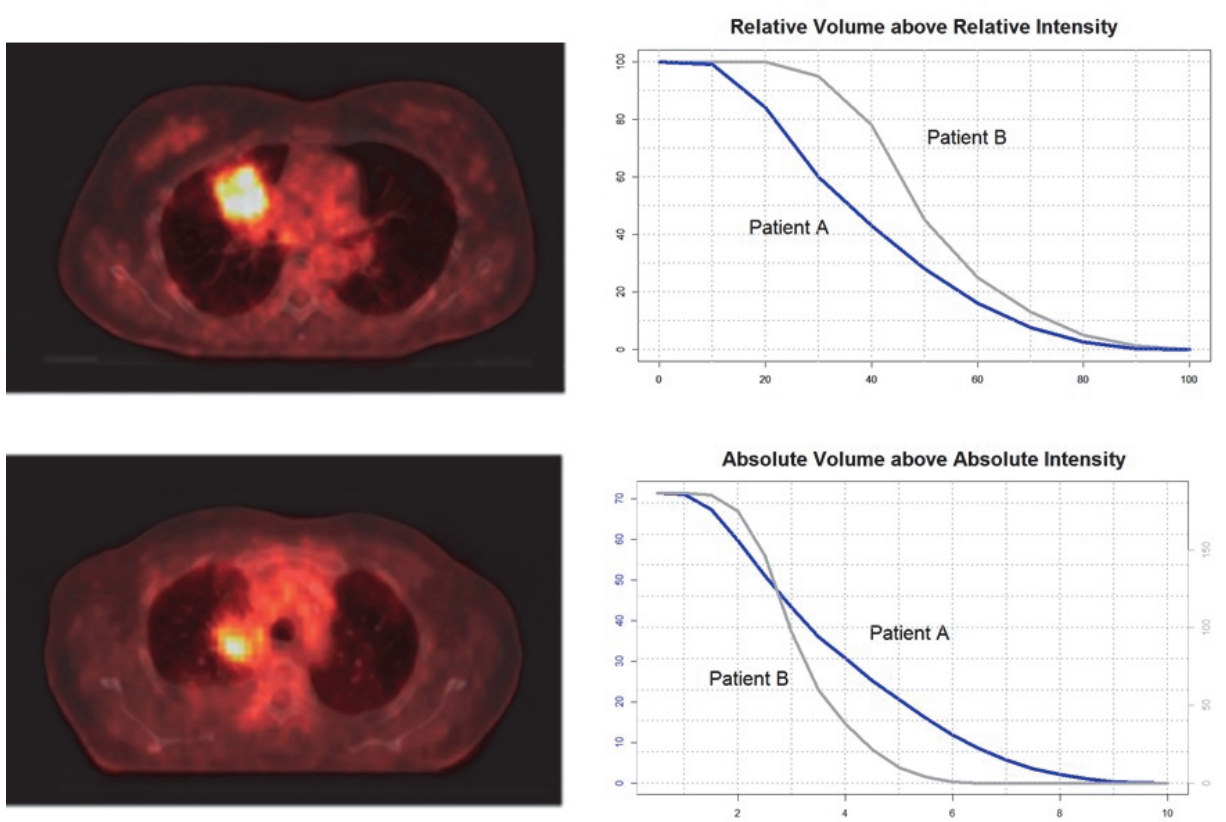

Figure 1 - Transverse view of CT-PET scan of NSCLC patients. Examples of Intensity-Volume Histograms for the two patients are shown on the right. Maximum SUV for patients $A$ and B (top and bottom left) are, respectively, 10.1 and 6.3, with corresponding volumes of 70 and $180 \mathrm{~cm}^{3}$ (derived from AVAl curves). From the RVRI curves, it can be inferred that the minimum SUV is around $8 \%(0.80)$ and $20 \%$ (1.25) of the maximum uptake. AVAl curves confirm that all the tumour volume is above these minimum uptake values for the corresponding patient.

\section{MATERIALS AND METHODS}

\section{Demographic and clinical data}

A total of 220 NSCLC (stage I-IIIB) patients referred to our institute between February 2005 and April 2011 were included. The study was approved by the appropriate Institutional Review Board and registered at clinicaltrials.gov (NCT00522639). Patients were treated with high dose radiotherapy (RT), had no surgery and no previous cancer within five years prior to diagnosis. Clinical follow-up was performed according to national guidelines.

\section{Radiotherapy}

All patients were treated at our institute with CT-based RT [10]. The XiO/Focal system (Computerized Medical Systems, St. Louis, MO, USA), based on a convolution-super- 
position algorithm with inhomogeneity corrections and according to ICRU 50 guidelines [11] was used for the RT planning.

Patients were treated according to three different regimens:

a. 44 patients were treated with the standard protocol for sequential chemo-radiation, as defined in August 2005 [12]. An individualized radiation dose escalation protocol (range 45 - 79.2 Gy) was delivered in two daily fractions of $1.8 \mathrm{~Gy}$. Dose escalation was limited by the mean lung dose or the spinal cord dose constraint. Between fractions, an eight hours interval was respected.

b. 148 patients received concurrent chemo-radiation. Following 2 cycles of carboplatin-gemcitabine, a radiation dose of $45 \mathrm{~Gy}$, in fractions of $1.5 \mathrm{~Gy}$ delivered twice a day for the first course, directly followed by an individualized dose ranging from 6-24 Gy and delivered in 2.0 Gy fractions once a day.

c. 28 patients received no chemotherapy treatment. The RT applied was analogous to the sequential protocol (range $54-79.2 \mathrm{~Gy}$ ).

\section{Imaging data acquisition}

All patients underwent a CT-PET scan before RT for treatment planning purposes (median: 7 days; range: 2 - 48). Patients fasted for at least 6 h before scanning. Up to December 2010, a total dose of (bodyweight x $4+20$ ) MBq of FDG was injected intravenously. After January 2011, the NEDPAS protocol was used and the administered dose was (2.5 x bodyweight) [13]. CT-PET images were acquired 60 minutes post injection.

Data acquired up to December 2006 were gathered on Siemens Biograph 16 CT-PET scanner. After January 2007 data were acquired on a Siemens Truepoint 40 CT-PET (Siemens AG, Munich, Germany). An Ordered Subset Expectation Maximization 2D 4 iterations 8 subsets (OSEM2D 4i8s) algorithm was used for PET image reconstruction. All PET scans were corrected for attenuation using the mid-ventilation phase of the $4 D C T$ or a 3DCT thorax in case the 4DCT was not of sufficient image quality due to irregular breathing of the patient. Model-based methods were applied for scatter correction. All exams were corrected for random events and decay.

\section{Tumour segmentation}

CT-PET images were imported into research treatment planning system Xio/Focal using the DICOM protocol. The primary Gross Tumour Volume (GTV) was delineated by experienced radiation oncologists on the fused CT-PET images and used as the region of interest (ROI) for further analysis. 


\section{Image processing and feature extraction}

SUV descriptors (maximum, mean and peak SUV), GTV and a set of metabolic features were extracted from the PET images and analysed. Although it is commonly regarded as a clinical feature, we computed GTV as an imaging feature and compared it against the ones in the same category and possible surrogates. Peak SUV was computed by means of a 3D kernel, representing a $1 \mathrm{~cm}^{3}$ sphere made on the same grid as the scan and centred on the maximum SUV voxel, as defined on the guidelines of PET Response Criteria in Solid Tumours (PERCIST), version 1.0 [14]. The uptake within this kernel was then averaged.

Metabolic features were based on the Intensity-Volume Histogram, which are analogous to the Dose-Volume histograms [15] and summarize in a curve the relation of tumour volume and SUV intensity. Five different curves can be defined: Absolute and Relative Volume above Relative Intensity threshold (AVRI and RVRI), Absolute and Relative Volume above Absolute Intensity threshold (AVAI and RVAI), and Absolute Intensity above Relative Volume threshold (AIRV). AVAI and RVAI are built in steps of 0.5 (SUV) up to the maximum uptake. Curves regarding relative quantities are built in steps of $10 \%$. Graphical representations of these types of curves for two patients are displayed in Figure 1. A total of 47 features were retrieved for the metabolic features: 9 for each set of curves based on relative thresholds (AVRI, RVRI and AIRV) and 10 for absolute thresholds (AVAI and RVAI), corresponding to a maximum absolute threshold of 5 . This threshold was chosen so as $85 \%$ of patients under analysis would have a valid feature.

Image analysis was performed in Matlab R2012b (The Mathworks, Natick, MA) using an adapted version of CERR (the Computational Environment for Radiotherapy Research) [16]. PET images were normalized to SUV before analysis. In-house developed image analysis software was used for feature extraction.

\section{Survival endpoint}

The primary endpoint was overall survival (OS), calculated from the start of RT until date of death or last follow-up. "Gemeentelijke Basis Administratie" (GBA), a decentralized population registration system of the Netherlands, was verified to assess survival status. A patient still alive at the end of the study was considered right-censored.

\section{Statistical analysis}

Imaging features based on PET scans were analysed using univariable Cox Proportional Hazard Regression. The assumption of proportional hazards was assessed by the Scaled Schoenfeld residuals. Time-dependent variables were constructed by adding interactions with $\log$ (time). A statistical significance at the $5 \%$ level was used. Estimated log hazard rations $(\log (H R))$, hazard ratios $(H R), p$-value and $95 \%$ confidence intervals $(95 \%$ 
$\mathrm{Cl})$ were reported. Additionally, a cluster analysis was performed to assess correlation between imaging features.

All statistical methods were implemented in $\mathrm{R}$ (version 2.15.0).

\section{RESULTS}

At the time of analysis 150 patients had died (70/220 right-censored). The median survival time assessed by Kaplan-Meier method was 1.81 years ( $95 \% \mathrm{Cl}: 1.54-2.19)$ ). Median follow-up time computed from start of RT until last follow-up was 1.47 years (95\% $\mathrm{Cl}: 1.20-1.70)$. Clinical information of patients in analysis is displayed on Table 1.

Table 1 - Clinical and demographic information of patients under analysis

\begin{tabular}{|c|c|}
\hline \multicolumn{2}{|l|}{ Age } \\
\hline (range) & $66.6 \pm 9.3(42.0-85.7)$ \\
\hline \multicolumn{2}{|l|}{ Sex } \\
\hline Male & $149(67.7 \%)$ \\
\hline Female & $71(32.3 \%)$ \\
\hline \multicolumn{2}{|l|}{ TNM stage } \\
\hline 1 & $12(5.5 \%)$ \\
\hline$\|$ & $15(6.8 \%)$ \\
\hline IIIA & $60(27.3 \%)$ \\
\hline IIIB & $105(47.7 \%)$ \\
\hline No information & $28(12.7 \%)$ \\
\hline \multicolumn{2}{|l|}{ Histology type } \\
\hline Squamous Cell Carcinoma & $69(31.3 \%)$ \\
\hline Adenocarcinoma & $36(16.4 \%)$ \\
\hline Large cells & $61(27.7 \%)$ \\
\hline Not otherwise specified (NOS) & $49(22.3 \%)$ \\
\hline Infiltrative carcinoma & $1(0.5 \%)$ \\
\hline No information & $4(1.8 \%)$ \\
\hline \multicolumn{2}{|l|}{ Chemotherapy } \\
\hline No & $28(12.7 \%)$ \\
\hline Sequential & $44(20 \%)$ \\
\hline Concurrent & $148(67.3 \%)$ \\
\hline
\end{tabular}

A univariable Cox regression was performed for the two types of PET features (SUV descriptors and IVH metrics). Out of the 47 IVH metrics, 9 showed statistical relevance, comprising AVAI curves from 0.5 up to 2.5 , AVRI up to $20 \%$ and RVRI of $80 \%$. None of 
the features from the AIRV and RVAl groups showed statistical significance. Detailed results of this analysis are displayed in Table 2.

In our study, maximum SUV showed no prognostic power for risk discrimination ( $p=$ 0.20). On the other hand, the portion of the tumour above $80 \%$ uptake $\left(\left.R V R\right|_{80 \%}\right)$ showed statistical significance $(p=0.05)$ and was associated with survival. The larger the relative volume above a higher uptake, the better the prognosis $(H R=0.11)$. This factor is correlated with the adjacent features describing fractions of tumour with uptakes above 70 and $90 \%$, however, none of these features showed statistical significance $\left(p\left(R V R I_{70 \%}\right)=0.07, P\left(R V R I_{90 \%}\right)=0.14\right)$.

Surrogates for GTV were encountered for metabolic features describing absolute volumes above lower absolute and relative uptakes (AVAI up to 2.5 and $\mathrm{AVRI}_{10 \%}$ ). None of the possible surrogates for MTV based on volumes above SUV of 3,40\% and 50\% of maximum SUV, showed significant associations with overall survival $\left(p\left(A V A I_{3}\right)=0.10, p\right.$ $\left.\left(\mathrm{AVAl}_{4}\right)=0.22, \mathrm{p}\left(\mathrm{AVRI}_{40 \%}\right)=0.15, \mathrm{p}\left(\left.\mathrm{AVRI}\right|_{50 \%}\right)=0.17\right)$

Detailed analysis was performed for GTV, maximum and peak SUV and RVRI80. Kaplan Meier curves and results for the log rank test based on a median split for these variables are displayed in Figure 2. GTV could discriminate between high- and low-risk patients $(p=0.05)$, while maximum $(p=0.25)$ and peak SUV $(p=0.08)$ and RVRI80 $(p=$ 0.13 ) could not, based on this median split.

The unsupervised cluster analysis revealed three distinct groups of related information Figure 3. While both AVAI and AVRI group well with volume, AIRV was found highly correlated with maximum, mean and peak SUV and grouped in a wider cluster. Similarly to those, no statistical significance was encountered for AIRV in our dataset. RVRI and RVAI features clustered in two independent groups. For simplicity reasons, only features presented on Table 1 were used for the clustering analysis. 
Table 2 - Cox regression statistics of imaging features in univariable analysis.

\begin{tabular}{|c|c|c|c|c|}
\hline & $\log (H R)$ & $\mathrm{HR}$ & p-value & $95 \% \mathrm{Cl} \mathrm{HR}$ \\
\hline \multicolumn{5}{|c|}{ Absolute Intensity above Relative Volume } \\
\hline $70 \%$ & 0.01 & 1.01 & 0.62 & $0.96-1.07$ \\
\hline $80 \%$ & 0.01 & 1.01 & 0.63 & $0.97-1.06$ \\
\hline $90 \%$ & 0.01 & 1.01 & 0.64 & $0.97-1.05$ \\
\hline \multicolumn{5}{|c|}{ Absolute Volume above Absolute Intensity } \\
\hline 0.5 & 0.00 & 1.00 & $0.01^{*}$ & $1.00-1.00$ \\
\hline 1.0 & 0.00 & 1.00 & $0.01^{*}$ & $1.00-1.00$ \\
\hline 1.5 & 0.00 & 1.00 & $0.01^{*}$ & $1.00-1.00$ \\
\hline \multicolumn{5}{|c|}{ Absolute Volume above Relative Intensity } \\
\hline $70 \%$ & 0.01 & 1.01 & 0.17 & $1.00-1.02$ \\
\hline $80 \%$ & 0.02 & 1.02 & 0.38 & $0.98-1.07$ \\
\hline $90 \%$ & 0.02 & 1.02 & 0.87 & $0.79-1.32$ \\
\hline \multicolumn{5}{|c|}{ Relative Volume above Absolute Intensity } \\
\hline 0.5 & -0.75 & 0.48 & 0.43 & $0.07-3.07$ \\
\hline 1.0 & 0.12 & 1.13 & 0.80 & $0.44-2.92$ \\
\hline 1.5 & 0.32 & 1.38 & 0.38 & $0.68-2.81$ \\
\hline \multicolumn{5}{|c|}{ Relative Volume above Relative Intensity } \\
\hline $70 \%$ & -1.32 & 0.27 & 0.08 & $0.06-1.16$ \\
\hline $80 \%$ & -2.19 & 0.11 & $0.05^{*}$ & $0.01-1.01$ \\
\hline $90 \%$ & -3.35 & 0.04 & 0.14 & $0.00-3.07$ \\
\hline Maximum SUV & 0.02 & 1.02 & 0.20 & $0.99-1.05$ \\
\hline Mean SUV & 0.03 & 1.03 & 0.44 & $0.96-1.10$ \\
\hline Peak SUV & 0.02 & 1.02 & 0.22 & $0.99-1.06$ \\
\hline Volume & 0.00 & 1.00 & $0.00 *$ & $1.00-1.00$ \\
\hline
\end{tabular}

* A statistical significance of $5 \%$ was used along the analysis 

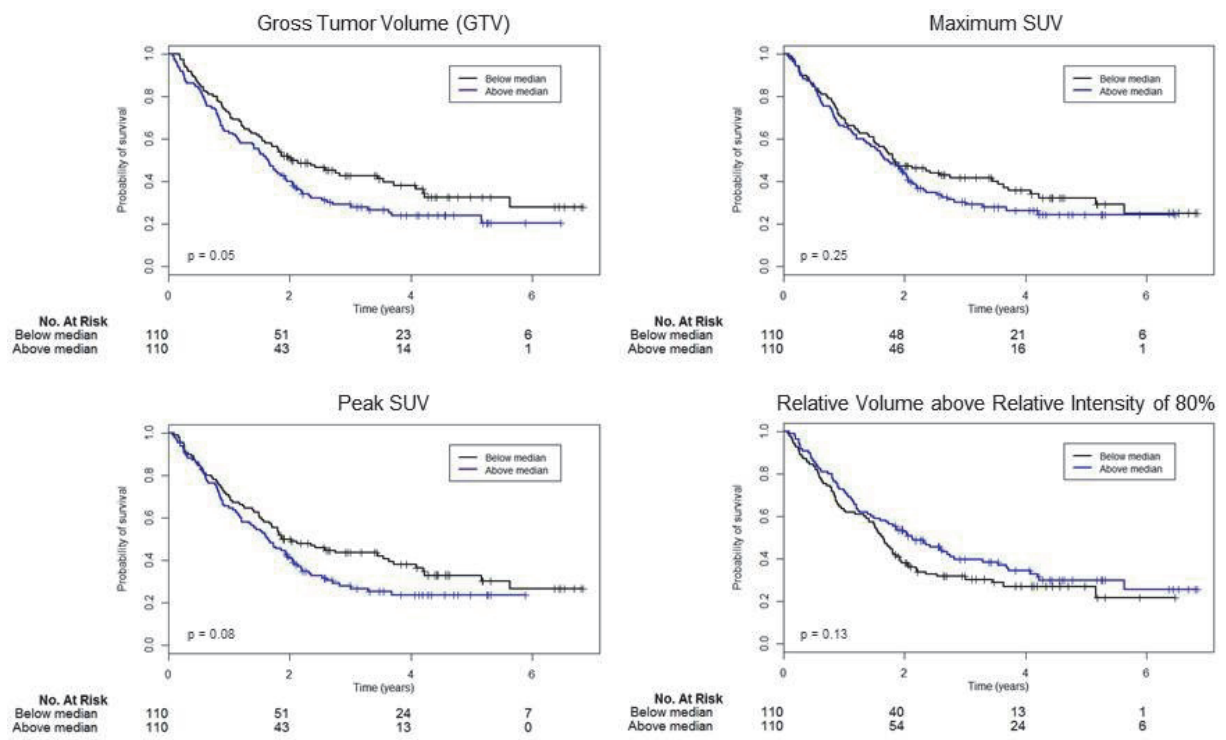

Figure 2 - Results for PET analysis: log rank test based on a median split for GTV (42.27), maximum SUV (10.12), peak SUV (7.92) and RVRI80 (0.04). Results for the log-rank test ( $p$-value) are displayed in the bottom left corner of each graph.

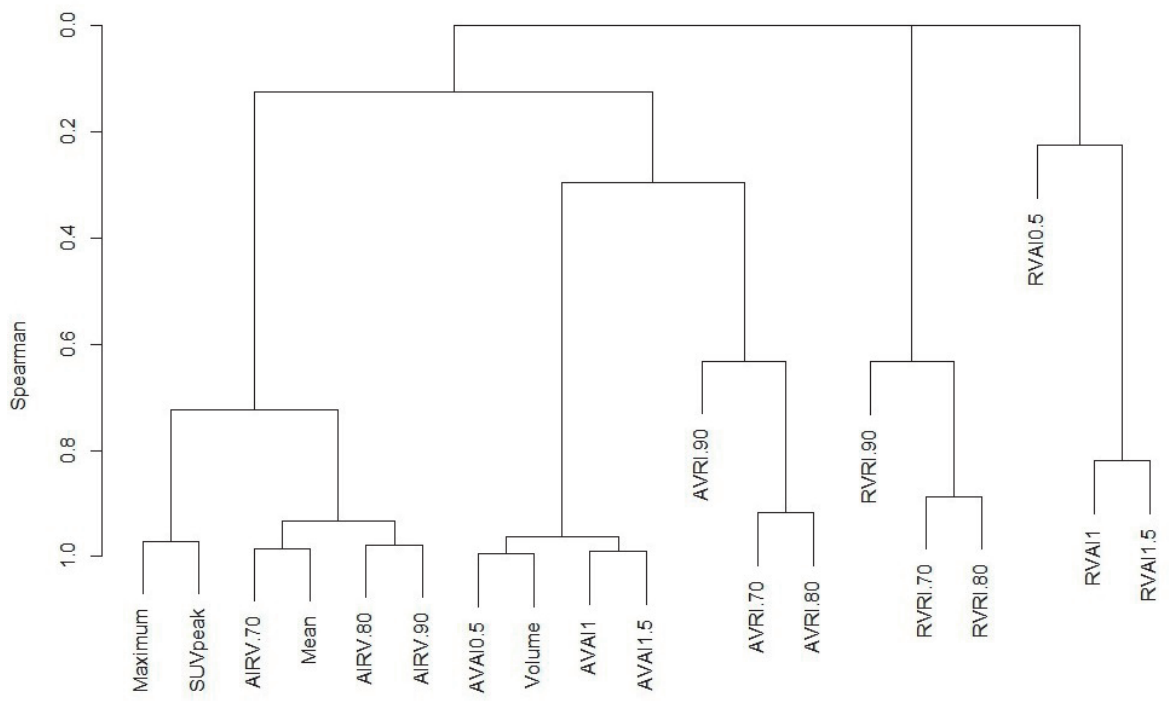

Figure 3 - Feature clustering based on Spearman correlation. For this clustering, only the features presented in Table 2 were used. It is evident from this diagram that the metabolic features aggregate in different cluster. Each of these clusters thus provides dissimilar information. 


\section{DISCUSSION}

Until now PET has mainly been used for staging purposes. This results of its sensitivity to detect distant and loco-regional lymph node metastasis. Some authors also reported the localization of areas with higher RT resistance based on pre-treatment PET [17]. Studies reported positive findings correlated with overall survival for pre-treatment PET scans [18], but relied mainly on simplistic metrics [19]. Also, metrics derived for tumour volume segmented by semi-automatic methods based on PET imaging, as MTV and TLG have been explored recently [6-9]. In our study a set of PET features was derived, described by the metabolic information based on the relation of FDG uptake and volume, and reported by IVH curves. Preliminary results revealed that these variables derived from PET imaging provided an added value for survival prediction of NSCLC patients treated with high dose RT.

There is reported evidence for the prognostic power of maximum SUV in early stage (I and II) NSCLC. However, there is insufficient evidence to draw the same conclusion for higher stage NSCLC patients [20-22]. In our cohort of patients, maximum SUV presented no statistical significant predictor of survival. This might be due to the large proportion of stage III patients in our cohort (75\%). An analysis performed for only stage III patients showed concordant results $(p=0.19)$. In fact, the analysis using only stage III patients showed no statistical power in the univariate analysis for any of the imaging features, except for volume $(p=0.02)$.

The tumour response using peak SUV is highly sensitive to the definition of its corresponding region of interest [23]. The non-prognostic power of peak SUV $(p=0.22)$ can be inferred from the fact that for such a small region of interest $(1 \mathrm{~cm} 3$ around the maximum SUV), there is an overall high correlation with maximum SUV ( $r=0.99)$.

Previous studies have described automatic delineation methods based on PET using either a fixed SUV or relative threshold. Cut-offs of, for example, 2.5, 3 and 4, and relative thresholds of $40 \%$ and $50 \%$ of the maximum SUV were already considered for the MTV definition [7]. Assuming the maximum uptake voxel is enclosed in the delineated GTV, $\mathrm{AVAl}_{2.5}, \mathrm{AVAl}_{3}$, and $\mathrm{AVAl}_{4}, \mathrm{AVRI}_{40 \%}$ and $\mathrm{AVRI}_{50 \%}$ were considered to be a good approximation of MTV. However, to compute TLG automatic segmentation must be performed as the mean SUV of MTV cannot be retrieved from the IVH curves.

To our knowledge, this is the first study reporting on portions of tumour volume above a relative uptake, as described by the RVRI and RVAI curves. These metrics ensure different volumes to be more comparable in terms of partial distribution of activity. Researchers have already reported the use of IVH metrics, but they did not include the relative features $[24,25]$ and their studies were based on smaller datasets and investigated other cancer sites. Two datasets of cervix and head and neck cancer treated with chemotherapy, were analysed using this approach by el Naqa [24]. Similarly, van Velden [25] analysed three lung cancer patients with dissimilar characteristics (hetero- 
geneous, homogeneous uptake and double acquisition, before and after one course of chemotherapy).

The analysis based on the IVH metrics showed for curves describing absolute volumes above lower uptakes $\left(p\left(\mathrm{AVAl}_{0.5}\right)<0.01, \mathrm{p}\left(\mathrm{AVAl}_{1}\right)<0.01, \mathrm{p}\left(\mathrm{AVAl}_{1.5}\right)=0.01\right)$, showed a good agreement with the results obtained for tumour volume $(p<0.05)$. Similarly, volume defined for lower relative SUV threshold (10\%) was also significant ( $p$ $=0.02$ ). Tumour volume was contoured by experienced oncologists based on CT-PET images. However, the use of these metrics instead of delineated volume might have been useful for a better feature analysis as it is less prone to variability as contouring by humans.

In our analysis, the relative portions of the tumour defined for higher relative uptakes showed a positive correlation with survival (RVRI80, $H R=0.11, p=0.05$ ). Unlike maximum and peak SUV, the higher the portion above higher uptake, the better the prognosis. Graphically, the same conclusion can be drawn from the curves dividing relative risk patients displayed in Figure 2 . This might be representative of a more homogeneous tumour for higher uptakes and therefore might be positively associated with better prognosis. AVRI metrics can be interpreted as RVRI multiplied by tumour volume. However, AVRI revealed an opposite effect and no statistical significance was found for the same thresholds.

We found several IVH metrics to be prognostic for overall survival. The patient population consisted of stage I-IIIB NSCLC patients, with a much higher proportion of later stages. Although it would be very interesting to draw conclusions for each stage group, we have not performed such an analysis due to the limited number of patients. Therefore, we plan to increase the dataset. Furthermore, automatic tumour segmentation is planned to validate the results obtained using the IVH approach for MTV and to compute TLG.

In conclusion, new approaches based on metabolic metrics derived from baseline PET images to assess response in NSCLC patients were analysed. Although we could not show that maximum SUV was prognostic for overall survival of the whole cohort, nor for a subgroup of stage III NSCLC patients, we were able to show that relative portions of tumour with higher SUV were positively associated with survival. This finding should be validated in new patient cohorts.

\section{ACKNOWLEDGEMENTS}

We acknowledge financial support from the CTMM framework (AIRFORCE project, $\mathrm{n}$ 030-103), EU 7th framework program (METOXIA, EURECA, ARTFORCE), euroCAT (IVA Interreg, www.eurocat.info), Radiomics (NIH, USA), EU IMI program (QuIC-ConCePT), NIH-QIN (Radiomics of NSCLC U01 CA143062) and the Dutch Cancer Society (KWF UM 2011-5020, KWF UM 2009-4454). 


\section{REFERENCES}

\{1] Travis WD, Brambilla E, Müller-Hermelink HK, Harris CC. World Health Organization Classification of Tumours: Pathology and Genetics of Tumours of the Lung, Pleura, Thymus and Heart. ed. Lyon, France: IARC Press, International Agency for Research on Cancer; 2004.

[2] Lambin P, van Stiphout RG, Starmans MH, Rios-Velazquez E, Nalbantov G, Aerts HJ, Roelofs E, van Elmpt W, Boutros PC, Granone P, Valentini V, Begg AC, De Ruysscher D, Dekker A. Predicting outcomes in radiation oncology--multifactorial decision support systems. Nat Rev Clin Oncol 2013;10: 27-40.

[3] Lambin P, Rios-Velazquez E, Leijenaar R, Carvalho S, van Stiphout RG, Granton P, Zegers CM, Gillies R, Boellard R, Dekker A, Aerts HJ. Radiomics: extracting more information from medical images using advanced feature analysis. Eur J Cancer 2012;48: 441-446.

[4] Salminen E, Mac Manus M. Impact of FDG-labelled positron emission tomography imaging on the management of non-small-cell lung cancer. Ann Med 2001;33: 404-409.

[5] Fuss M. Strategies of assessing and quantifying radiation treatment metabolic tumour response using F18 F DG Positron Emission Tomography (PET). Acta Oncol 2010;49: 948-955.

[6] Cook GJ, Yip C, Siddique M, Goh V, Chicklore S, Roy A, Marsden P, Ahmad S, Landau D. Are Pretreatment 18F-FDG PET Tumour Textural Features in Non-Small Cell Lung Cancer Associated with Response and Survival After Chemoradiotherapy? J Nucl Med 2013;54: 19-26.

[7] Van de Wiele C, Kruse V, Smeets P, Sathekge M, Maes A. Predictive and prognostic value of metabolic tumour volume and total lesion glycolysis in solid tumours. Eur J Nucl Med Mol Imaging 2013;40: 290301.

[8] Hyun SH, Choi JY, Kim K, Kim J, Shim YM, Um SW, Kim H, Lee KH, Kim BT. Volume-Based Parameters of 18F-Fluorodeoxyglucose Positron Emission Tomography/Computed Tomography Improve Outcome Prediction in Early-Stage Non-Small Cell Lung Cancer After Surgical Resection. Ann Surg 2013;257: 364-370.

[9] Soussan M, Chouahnia K, Maisonobe JA, Boubaya M, Eder V, Morere JF, Buvat I. Prognostic implications of volume-based measurements on FDG PET/CT in stage III non-small-cell lung cancer after induction chemotherapy. Eur J Nucl Med Mol Imaging 2013.

[10] Prescribing, Recording, and Reporting Photon Beam Therapy (Report 50). In: International Commission on Radiation Units and Measurements, 1993.

[11] ICRU Report 50: Prescribing, Recording, and Reporting Photon Beam Therapy. In. Bethesda, MD: International Commission on Radiation Units and Measurements, 1993.

[12] Oncology MR. Radiotherapy for NSCLC to a Individualized MLD (BRONC MLD). In, 2010.

[13] Boellaard R, Oyen WJ, Hoekstra CJ, Hoekstra OS, Visser EP, Willemsen AT, Arends B, Verzijlbergen FJ, Zijlstra J, Paans AM, Comans EF, Pruim J. The Netherlands protocol for standardisation and quantification of FDG whole body PET studies in multi-centre trials. Eur J Nucl Med Mol Imaging 2008;35: 2320-2333.

[14] Wahl RL, Jacene H, Kasamon Y, Lodge MA. From RECIST to PERCIST: Evolving Considerations for PET response criteria in solid tumours. J Nucl Med 2009;50 Suppl 1: 122S-150S.

[15] Drzymala RE, Mohan R, Brewster L, Chu J, Goitein M, Harms W, Urie M. Dose-volume histograms. Int J Radiat Oncol Biol Phys 1991;21: 71-78.

[16] Deasy JO, Blanco Al, Clark VH. CERR: a computational environment for radiotherapy research. Medical Physics 2003;30: 979-985.

[17] Aerts HJ, van Baardwijk AA, Petit SF, Offermann C, Loon J, Houben R, Dingemans AM, Wanders R, Boersma L, Borger J, Bootsma G, Geraedts W, Pitz C, Simons J, Wouters BG, Oellers M, Lambin P, Bosmans G, Dekker AL, De Ruysscher D. Identification of residual metabolic-active areas within individual NSCLC tumours using a pre-radiotherapy (18)Fluorodeoxyglucose-PET-CT scan. Radiother Oncol 2009;91: 386392.

[18] Kauppi JT, Oksala N, Salo JA, Helin H, Karhumaki L, Kemppainen J, Sihvo EI, Rasanen JV. Locally advanced esophageal adenocarcinoma: response to neoadjuvant chemotherapy and survival predicted by ([18F])FDG-PET/CT. Acta Oncol 2012;51: 636-644. 


\section{CHAPTER 4}

[19] Velazquez ER, Aerts HJ, Oberije C, De Ruysscher D, Lambin P. Prediction of residual metabolic activity after treatment in NSCLC patients. Acta Oncol 2010;49: 1033-1039.

[20] Abelson JA, Murphy JD, Trakul N, Bazan JG, Maxim PG, Graves EE, Quon A, Le QT, Diehn M, Loo BW, Jr. Metabolic imaging metrics correlate with survival in early stage lung cancer treated with stereotactic ablative radiotherapy. Lung Cancer 2012;78: 219-224.

[21] Agarwal M, Brahmanday G, Bajaj SK, Ravikrishnan KP, Wong CY. Revisiting the prognostic value of preoperative (18)F-fluoro-2-deoxyglucose ( (18)F-FDG) positron emission tomography (PET) in early-stage (I \& II) non-small cell lung cancers (NSCLC). Eur J Nucl Med Mol Imaging 2010;37: 691-698.

[22] Hoang JK, Hoagland LF, Coleman RE, Coan AD, Herndon JE, 2nd, Patz EF, Jr. Prognostic value of fluorine18 fluorodeoxyglucose positron emission tomography imaging in patients with advanced-stage nonsmall-cell lung carcinoma. J Clin Oncol 2008;26: 1459-1464.

[23] Vanderhoek M, Perlman SB, Jeraj R. Impact of the definition of peak standardized uptake value on quantification of treatment response. J Nucl Med 2012;53: 4-11.

[24] El Naqa I, Grigsby P, Apte A, Kidd E, Donnelly E, Khullar D, Chaudhari S, Yang D, Schmitt M, Laforest R, Thorstad W, Deasy JO. Exploring feature-based approaches in PET images for predicting cancer treatment outcomes. Pattern Recognit 2009;42: 1162-1171.

[25] van Velden FH, Cheebsumon P, Yaqub M, Smit EF, Hoekstra OS, Lammertsma AA, Boellaard R. Evaluation of a cumulative SUV-volume histogram method for parameterizing heterogeneous intratumoural FDG uptake in non-small cell lung cancer PET studies. Eur J Nucl Med Mol Imaging 2011;38: 1636-1647. 


\section{Chapter}

\section{Stability of FDG-PET Radiomics features: An integrated analysis of test-retest and inter-observer variability}

Published in: Acta Oncologica, 52(7), 2013 Oct:1391-7

Stability of FDG-PET Radiomics features: An integrated analysis of test-retest and interobserver variability

Ralph T. H. Leijenaar*, Sara Carvalho*, Emmanuel Rios Velazquez, Wouter J. C. van Elmpt, Chintan Parmar, Otto S. Hoekstra, Corneline J. Hoekstra, Ronald Boellard, André L. A. J. Dekker, Robert J. Gillies, Hugo J. W. L. Aerts, and Philippe Lambin

* These authors contributed equally to this work 


\section{ABSTRACT}

\section{Background}

Besides basic measurements as maximum standardized uptake value (SUV)max or SUVmean derived from ${ }^{18}$ F-FDG positron emission tomography (PET) scans, more advanced quantitative imaging features (i.e. "Radiomics" features) are increasingly investigated for treatment monitoring, outcome prediction, or as potential biomarkers. With these prospected applications of Radiomics features, it is a requisite that they provide robust and reliable measurements. The aim of our study was therefore to perform an integrated stability analysis of a large number of PET-derived features in non-small cell lung carcinoma (NSCLC), based on both a test-retest and an inter-observer setup.

\section{Methods}

Eleven NSCLC patients were included in the test-retest cohort. Patients underwent repeated PET imaging within a one-day interval, before any treatment was delivered. Lesions were delineated by applying a threshold of $50 \%$ of the maximum uptake value within the tumour. Twenty-three NSCLC patients were included in the inter-observer cohort. Patients underwent a diagnostic whole body PET-computed tomography (CT). Lesions were manually delineated based on fused PET-CT, using a standardized clinical delineation protocol. Delineation was performed independently by five observers, blinded to each other. Fifteen first order statistics, 39 descriptors of intensity volume histograms, eight geometric features and 44 textural features were extracted. For every feature, test-retest and inter-observer stability was assessed with the intra-class correlation coefficient (ICC) and the coefficient of variability, normalized to mean and range. Similarity between test-retest and inter-observer stability rankings of features was assessed with Spearman's rank correlation coefficient.

\section{Results}

Results showed that the majority of assessed features had both a high test-retest (71\%) and inter-observer (91\%) stability in terms of their ICC. Overall, features more stable in repeated PET imaging were also found to be more robust against inter-observer variability.

\section{Conclusion}

Results suggest that further research of quantitative imaging features is warranted with respect to more advanced applications of PET imaging as being used for treatment monitoring, outcome prediction or imaging biomarkers. 


\section{INTRODUCTION}

Positron emission tomography (PET) has been shown to be a valuable tool for the detection and staging of lung cancer [1]. In recent years also PET imaging has also been increasingly used for treatment planning [2] and response monitoring in radiotherapy [3]. The most widely used tracer in oncological PET imaging is the glucose analog ${ }^{18} \mathrm{~F}$ Fluoro-2-Deoxy-D-glucose (FDG), commonly quantified by the standardized uptake value (SUV) [4]. Previous research provides evidence of basic and easily derived pretreatment PET measurements, such as the maximum (SUVmax) or mean SUV (SUVmean), being predictors for treatment outcome in NSCLC [5-7]. Besides these basic measurements, more advanced quantitative imaging features are increasingly investigated for treatment monitoring and outcome prediction in lung and other cancer sites [8-10], or as potential imaging biomarkers [11].

The use of basic and more advanced descriptors derived from PET imaging is within the scope of "Radiomics" [12-14]: a high throughput approach to extract and mine a large number of quantitative features from medical images, where it is hypothesized that it will improve tumour characterization and treatment outcome prediction. However, with the prospect of using these Radiomics features for future prognostic and predictive models, knowledge about their reliability and variability is needed. A few recent studies have investigated these aspects of FDG-PET derived parameters in different cancer sites, including the test-retest stability of basic SUV measurements [15], testretest stability of a number of basic and textural features [16], or the variability of textural features due to image acquisition and reconstruction parameters [17]. However, to our knowledge no previous study has performed an integrated stability analysis of a large number of PET features in NSCLC, based on both a test-retest and an interobserver setup. Therefore, the aim our study is to independently examine the feature's test-retest reliability and inter-observer stability between multiple manual tumour delineations. Moreover, we aim to combine the information obtained from both analyses to assess if imaging features that are more stable in repeated PET imaging are also more robust against inter-observer variability. Based on literature research, we strived to include a broad collection of PET based imaging features used in the context of predictive and/or prognostic modelling in cancer, to provide a comprehensive overview.

\section{MATERIALS AND METHODS}

This study includes two separate patient cohorts in order to assess both the test-retest and inter-observer variability of a large number of quantitative imaging features. All patients signed an informed consent form in accordance with approval by the institutional review board. A schematic representation of the workflow applied in our study is depicted in Figure 1. 


\section{Test-retest cohort}

Eleven patients with histology- or cytology-diagnosed non-small cell lung cancer (NSCLC) were included in this patient cohort, as described in [18]. Patients underwent two baseline ${ }^{18}$ F-FDG-PET scans within a one-day interval, before any treatment was delivered. PET images were acquired on an ECAT EXACT HR1 scanner (Siemens/CTI) and iteratively reconstructed using normalization- and attenuation-weighted orderedsubset expectation maximization with two iterations and sixteen subsets (OSEM 2i16s). All images had an in-plane resolution of $5.15 \times 5.15 \mathrm{~mm} /$ pixel and a slice thickness of $2.43 \mathrm{~mm}$. Further patient and imaging details are described by Frings et al [18]. Lesions with adequate uptake were first identified and subsequently delineated by applying a threshold of $50 \%$ of the maximum uptake value within the tumour [19], using a semiautomatic delineation tool [18] (Figure 2).

\section{Inter-observer cohort}

Twenty-three patients with histologically proven NSCLC were included in this patient cohort, as described previously in [20]. Patients underwent a diagnostic whole body PET-CT scan acquired on a SOMATOM Sensation 16 with an ECAT ACCEL PET scanner (Siemens, Erlangen, Germany). PET images were iteratively reconstructed using normalization- and attenuation-weighted OSEM 4i8s. Images had an in-plane resolution of $5.31 \times 5.31 \mathrm{~mm} /$ pixel and a $5 \mathrm{~mm}$ slice thickness. Primary tumours and involved lymph nodes were identified and manually delineated based on fused PET-CT images, using a standardized clinical delineation protocol. Delineation of the lesions was performed independently by five observers and all observers were blinded to the contours delineated by the others (Figure 2.C-D). Manual delineations were performed on XiO/Focal (Computer Medical System, Inc., St. Louis, MO). For further details on the patient cohort, imaging and delineation, we refer to the publication of van Baardwijk et al [20].

\section{Image processing and feature extraction}

All image analysis was performed in Matlab R2012b (The Mathworks, Natick, MA) using an adapted version of CERR (the Computational Environment for Radiotherapy Research) [21] extended with in-house developed Radiomics image analysis software to extract imaging features. PET images and delineated VOls were first imported into CERR, where the image intensities were normalized to SUV [4]. First order statistics consisted of basic SUV measurements and features describing histogram of voxel intensity values contained within the VOI. 


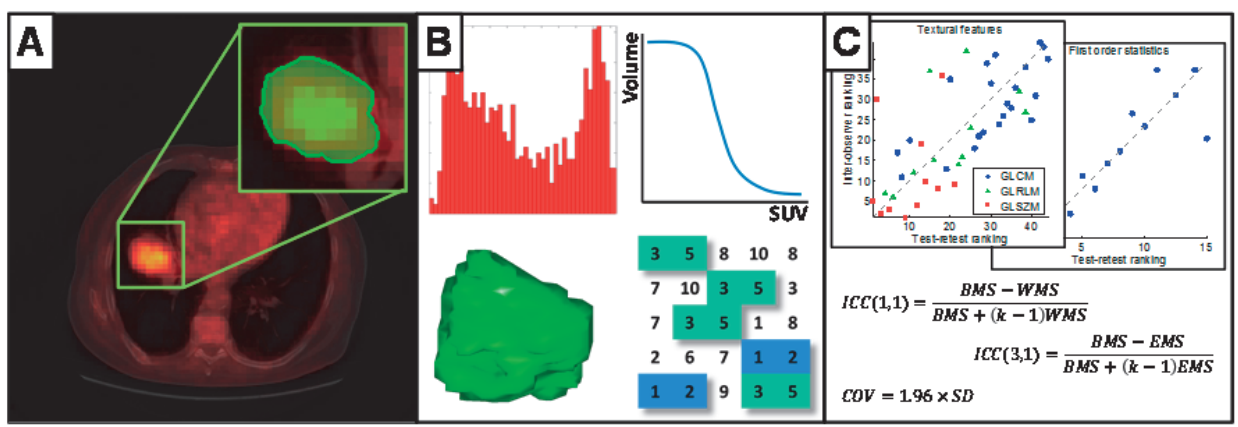

Figure 1 - Schematic of the workflow applied in our study: (A) Acquisition of PET images (fused CT for illustrative purposes), followed by tumour delineation; (B) Extraction of Radiomics features from the defined volume of interest; (C) Test-retest and inter-observer stability analysis.

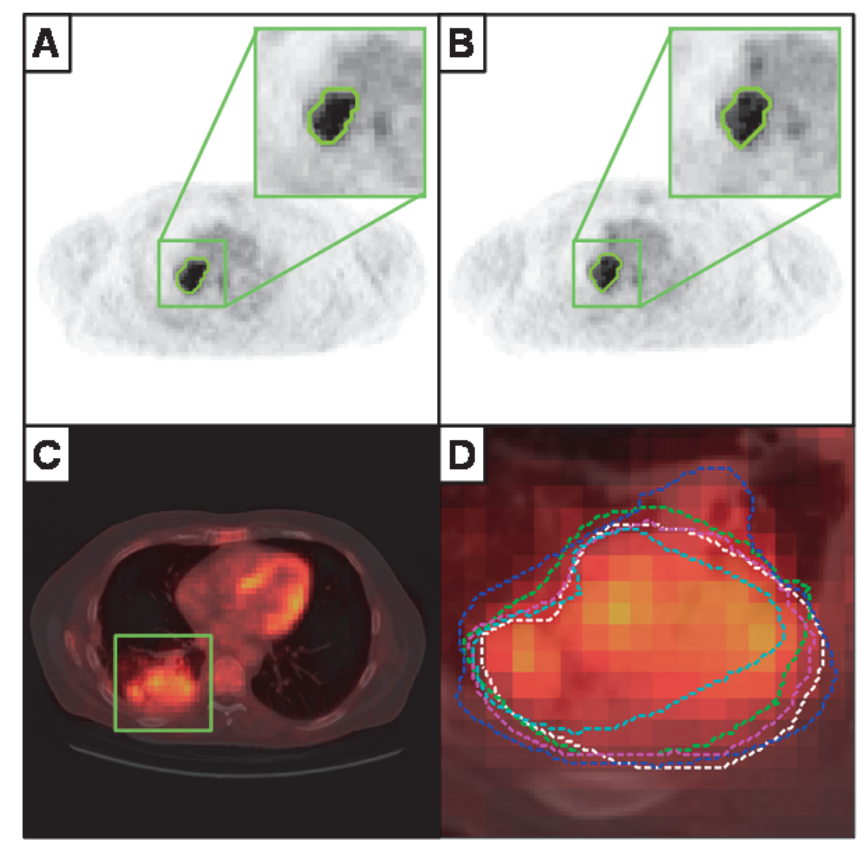

Figure 2 - (A and B) Representative images of repeated imaging of a patient from the test-retest cohort, with the 50\% SUVmax tumour delineation shown outlined in green, for respectively the first and second baseline PET scan. (C) Representative image of a patient from the inter-observer cohort, where the lesion area is outlined with the green square (fused CT for illustrative purposes); (D) Enlargement of the lesion area with in different colours the five independent tumour delineations by multiple observers.

A set of metrics was derived from intensity volume histogram (IVH) representations [10], which summarize the complex three dimensional data contained in the image into a single curve, allowing for a simplified interpretation. Three IVH definitions were considered: the relative volume as a function of the relative intensity (RVRIx), the absolute 
volume as a function of the relative intensity (AVRIx) and the intensity threshold as a function of the relative volume having a maximum intensity lower than the threshold (AIRVx). Relative steps in volume and intensity $(\mathrm{x})$ were taken in $10 \%$ increments, from 10\%-90\%. Furthermore, three differential IVH metrics were considered: RVRIXRVRI(100-x), AVRIx-AVRI(100-x), and AIRVx-AIRV(100-x).

Geometric features were calculated, describing the three-dimensional shape and size of the lesions. Textural features describing patterns or spatial distribution of voxel intensities, were calculated from respectively grey level co-occurrence (GLCM) [22], grey level run-length (GLRLM) [23] and grey level size-zone texture matrices (GLSZM) [9]. Determining texture matrix representations requires the voxel intensity values within the VOI to be discretized. Voxel intensities were therefore resampled into equally spaced bins using a bin-width of 0.5 units SUV. This discretization step not only reduces image noise, but also normalizes intensities across all patients, allowing for a direct comparison of all calculated textural features between patients. Texture matrices were determined considering 26-connected voxels (i.e. voxels were considered to be neighbours in all 13 directions in three dimensions) and a distance of one voxel between consecutive voxels was set for co-occurrence and grey level run-length matrices. Features derived from co-occurrence and grey level run-length matrices were calculated by averaging their value over all 13 considered directions in three dimensions.

Overall, the extracted imaging features comprised 15 first order statistics, 39 descriptors of intensity volume histograms, 8 geometric features and 44 textural features.

\section{Statistical Analysis}

The intra-class correlation coefficient (ICC) [24] was calculated to provide an indication of both the test-retest and inter-observer reliability of feature measurements. The ICC is a statistical measure between 0 and 1 , where 0 indicates no and 1 indicates perfect reliability. To determine the ICC, variance estimates were obtained through partitioning the total variance by means of non-parametric analysis of variance (ANOVA) by ranks. To assess test-retest reliability of imaging features, we used the definition of $\operatorname{ICC}(1,1)$, given by:

$$
\operatorname{ICC}(1,1)=\frac{B M S-W M S}{B M S+(k-1) W M S}
$$

Where BMS and WMS are respectively the between-subjects and within-subjects mean squares, obtained by Kruskal-Wallis one-way ANOVA, and $k$ is the number of repeated measurements (i.e. PET scans). Inter-observer stability was determined with the definition of $\operatorname{ICC}(3,1)$, with the form:

$$
\operatorname{ICC}(3,1)=\frac{B M S-E M S}{B M S+(k-1) E M S}
$$


Where BMS and EMS are the between-subjects and residual mean squares acquired from Friedman's two-way ANOVA, and $k$ is the number of observers (i.e. delineators). Absolute variability was estimated as the coefficient of variability (COV), defined as the value below which the difference between two measurements will be with 95\% probability [25]:

$$
\operatorname{COV}=1,96 \times S D
$$

Where SD is the standard deviation for single differences on different subjects (i.e. lesions). To provide a basis for evaluating the magnitude of the test-retest and interobserver COV values, we normalized them to a percentage of the mean feature value $\left(\mathrm{COV}_{\% \text { mean }}\right)$ as well as the range of feature values (2.5 - 97.5 percentile; $\mathrm{COV}_{\% \text { range }}$ ) over all included lesions. To assess the similarity of the test-retest and inter-observer stability rankings of features we ranked them, per feature group, in terms of their ICC. The similarity of feature rankings was determined with Spearman's rank correlation coefficient $\left(\rho_{S}\right)$.

All statistical analysis was performed in Matlab R2012b (The Mathworks, Natick, MA).

\section{RESULTS}

Lesion identification and delineation resulted in a total number of 18 lesions to be included for the test-retest analysis and respectively 27 lesions for the inter-observer analysis. Test-retest and inter-observer ICC, COV \%mean and COV \%range values are summarized per feature group in respectively Table 1 and Table 2, where we classified features into three groups, as having a high (ICC $\geq 0.8)$, medium $(0.8>I C C \geq 0.5)$, or low $(I C C<0.5)$ stability.

AVRIx and RVRIx for $x \leq 50 \%$ were excluded from test-retest analysis, since they represent the entire (relative) tumour volume and therefore provide no additional information on test-retest variability. In summary, $71 \%$ of all assessed features had high, $18 \%$ a medium and $11 \%$ a low stability in terms of their test-retest ICC. We found a high inter-observer stability for $91 \%$ of imaging features, whereas $8 \%$ and $1 \%$ of the features had a medium or respectively low stability. As expected, SUV $\max$ and SUV $V_{\text {peak }}$ showed perfect inter-observer stability $(I C C=1)$. Because of the same reasoning outlined above, we also excluded RVRIx and AVRIx for $x \leq 50 \%$ from the comparative analysis. Scatter plots of stability rankings for every feature group are depicted in Figure 3.A-D. Considering all features, we observed a good overall similarity in feature stability rankings in terms of test-retest and inter-observer ICCS $\left(\rho_{s}=0.67, p<0.01\right)$. Comparing stability rankings per feature group, we found a high similarity for both the first order statistics $\left(\rho_{s}=0.88, p<0.01\right)$ and the textural features $\left(\rho_{s}=0.72, p<0.01\right)$.

As can be observed from Figure 3.D, features based on GLSZM have the overall lowest ranks in both analyses, indicating these features have the highest variability amongst 


\section{CHAPTER 5}

all textural features. For the IVH features the observed similarity was more moderate $\left(\rho_{s}=0.57, p<0.01\right)$. Comparing the rankings for the geometric features resulted in a nonsignificant $\rho_{s}$ of $0.66(p=0.09)$. However, from Figure 3.C, a positive trend in similarity can be observed. Overall, these results show that features that are more stable in repeated PET imaging are also more robust against inter-observer variability.

Table 1 - Results for the test-retest analysis, showing ICC, COV \%mean and COV\%range ranges, as well as the number of features per feature group and per class, defined as high (ICC $\geq 0.8)$, medium $(0.8>I C C \geq 0.5)$, or low $(\mathrm{ICC}<0.5)$ stability. Median values of ICC, COV \%mean and $\mathrm{COV}_{\% \text { range }}$ ranges are shown within brackets.

\begin{tabular}{|c|c|c|c|c|}
\hline Stability class & $\mathrm{N}$ & ICC & $\mathrm{COV}_{\% \text { mean }}(\%)$ & $\mathrm{COV}_{\% \text { range }}(\%)$ \\
\hline \multicolumn{5}{|l|}{ First order statistics } \\
\hline High stability & 13 & $0.81-0.96(0.92)$ & $17.27-86.29(23.45)$ & $12.22-35.36(14.67)$ \\
\hline Medium stability & 0 & - & - & - \\
\hline Low stability & 2 & $0.27-0.28(0.27)$ & $57.29-110.48(83.89)$ & $55.61-60.58(58.09)$ \\
\hline \multicolumn{5}{|l|}{ IVH features } \\
\hline High stability & 18 & $0.80-0.94(0.86)$ & 17.09 - 44.07 (29.19) & $3.39-23.78(14.82)$ \\
\hline Medium stability & 3 & $0.61-0.78(0.77)$ & $37.26-105.65(50.40)$ & $6.03-28.04(20.33)$ \\
\hline Low stability & 8 & $0.00-0.48(0.27)$ & 7.68 - $99.25(46.30)$ & $46.82-68.00(60.54)$ \\
\hline \multicolumn{5}{|l|}{ Geometric features } \\
\hline High stability & 8 & $0.81-0.88(0.83)$ & $12.25-37.61(29.53)$ & $3.80-31.58(18.79)$ \\
\hline Medium stability & 0 & - & - & - \\
\hline Low stability & 0 & - & - & - \\
\hline \multicolumn{5}{|l|}{ Textural features } \\
\hline High stability & 29 & $0.81-0.93(0.89)$ & $2.76-166.45$ (36.90) & $5.94-36.42(19.25)$ \\
\hline Medium stability & 14 & $0.54-0.79(0.64)$ & $3.57-465.37(75.93)$ & $4.32-54.56(33.96)$ \\
\hline Low stability & 1 & 0.35 & 84.19 & 53.59 \\
\hline
\end{tabular}


Table 2 - Results for the inter-observer analysis, showing ICC, COV \%mean and COV\%range ranges, as well as the number of features per feature group and per class, defined as high (ICC $\geq 0.8)$, medium $(0.8>I C C \geq 0.5)$, or low $(I C C<0.5)$ stability. Median values of ICC, COV \%mean and $\mathrm{COV}_{\% \text { range }}$ ranges are shown within brackets.

\begin{tabular}{|c|c|c|c|c|}
\hline Stability class & $\mathrm{N}$ & ICC & $\mathrm{COV}_{\% \text { mean }}(\%)$ & $\mathrm{COV}_{\% \text { range }}(\%)$ \\
\hline \multicolumn{5}{|l|}{ First order statistics } \\
\hline High stability & 14 & $0.87-1.00(0.98)$ & $2.07-58.17(15.25)$ & $1.20-22.75$ (7.39) \\
\hline Medium stability & 1 & 0.79 & 65.81 & 41.21 \\
\hline Low stability & 0 & - & - & - \\
\hline \multicolumn{5}{|l|}{ IVH features } \\
\hline High stability & 34 & $0.82-1.00(0.97)$ & $5.60-131.45(28.57)$ & $1.23-52.15(10.70)$ \\
\hline Medium stability & 5 & $0.63-0.77(0.72)$ & $4.53-39.04(21.74)$ & $38.72-57.65(51.14)$ \\
\hline Low stability & 0 & - & - & - \\
\hline \multicolumn{5}{|l|}{ Geometric features } \\
\hline High stability & 8 & $0.80-0.98(0.97)$ & $11.63-48.47(26.79)$ & $9.60-31.31(19.20)$ \\
\hline Medium stability & 0 & - & - & - \\
\hline Low stability & 0 & - & - & - \\
\hline \multicolumn{5}{|l|}{ Textural features } \\
\hline High stability & 39 & $0.80-0.99(0.95)$ & $1.20-257.20(28.61)$ & $5.34-40.03(13.19)$ \\
\hline Medium stability & 3 & $0.50-0.77(0.75)$ & $44.46-128.87(104.07)$ & $12.38-51.25(30.16)$ \\
\hline Low stability & 2 & $0.17-0.19(0.18)$ & $156.41-192.86(174.63)$ & $57.96-76.36(67.16)$ \\
\hline
\end{tabular}




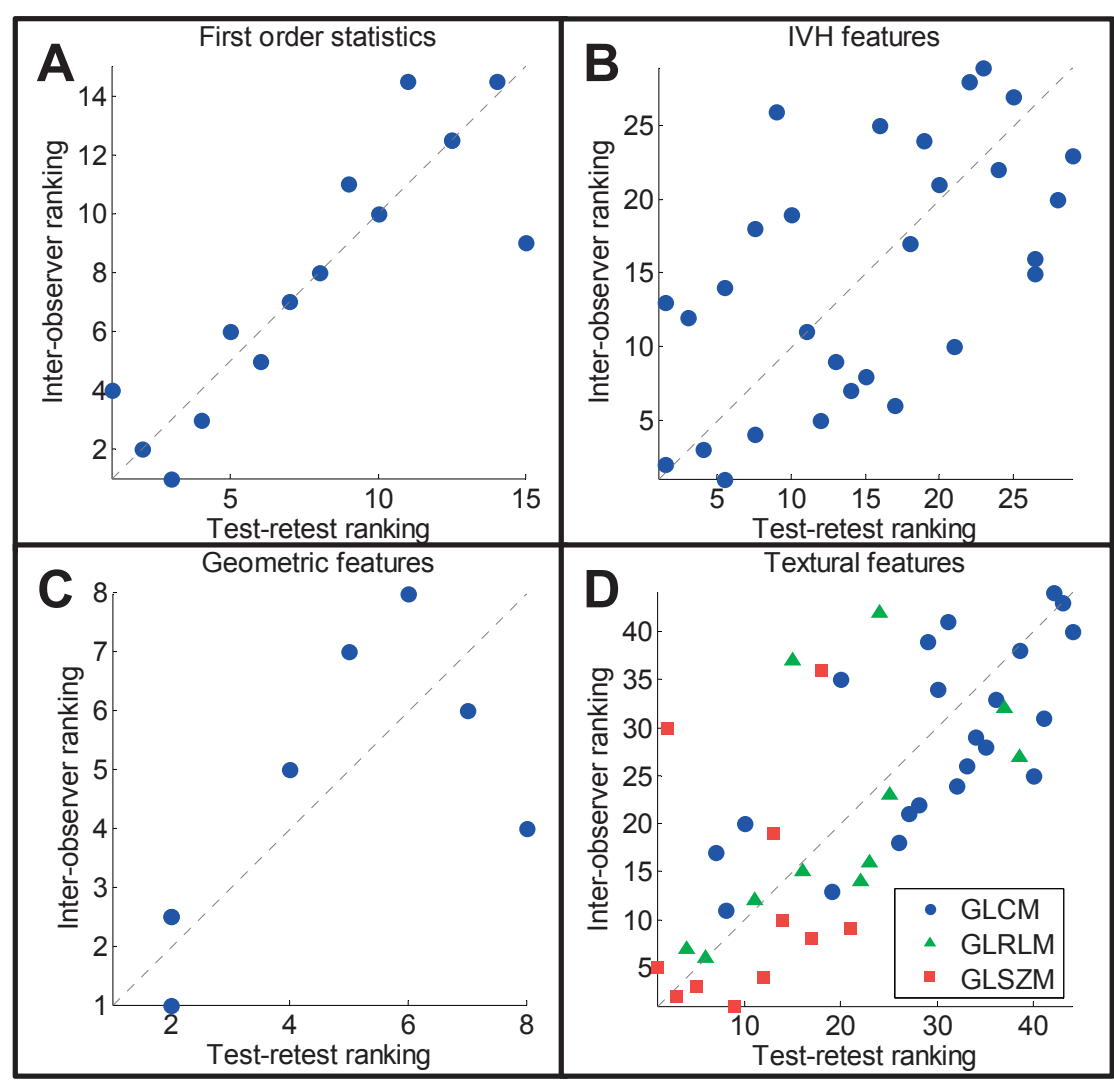

Figure 3 - Scatter plots of stability rankings of test-retest versus inter-observer intra-class correlation coefficients. Plotted diagonal illustrates perfect correlation. (A) First order statistics $\left(\rho_{s}=0.877, p<<0.001\right)$. (B) Intensity volume histogram features $\left(\rho_{s}=0.572, p<<0.001\right)$. (C) Geometric features $\left(\rho_{s}=0.663, p<<0.001\right)$. (D) Textural features ( $\left.\rho_{\mathrm{s}}=0.719, \mathrm{p}<<0.001\right)$, with GLCM features in blue circles, GLRLM features in green triangles and GLSZM features in red squares.

\section{DISCUSSION}

Increased investigation of quantitative imaging features to monitor response to treatment, treatment outcome or as potential imaging biomarkers, raised the requisite to validate their accuracy, robustness and stability. We first independently investigated the stability of imaging features in both a test- retest and intra-observer setting and subsequently performed an integrated analysis. Our results indicated high ICC values and high stability for the majority of assessed PET image features in both the test-retest (71\%) and inter-observer analysis (91\%). Furthermore, we found that features that were more stable in repeated imaging were also more robust against multiple tumour delineations. 
These results suggest that, even though there are different sources of feature variability, one can define a set of features being overall most reliable.

We focused our results mainly on the ICC. Being a dimensionless statistic, the ICC is useful when comparing the stability of measures with different units, as is the case with the PET imaging features assessed in this study. We chose arbitrary ICC thresholds to define high, medium and low stability. There is however no consensus how high the ICC should be to for a measure to be considered to have an acceptably high reliability, since the ICC is a relative measure determined from the between and within subject (i.e. lesion) variance, which makes it a sample specific measure. This implies that ICC values obtained from our test-retest analysis were not directly comparable to those from the inter-observer analysis, since they were independently obtained from two different patient cohorts (i.e. different lesions and differences in image acquisition and reconstruction). To overcome this limitation, we ranked features according to their ICC, allowing us to compare stability rankings of features between the two analyses.

In the inter-observer analysis, SUVmax and SUVpeak both had an ICC of 1, indicating perfect stability. However, we did observe a small COV for these features, which was unexpected. A detailed look into all delineations revealed that for only one lesion, one delineator did not include the maximum uptake voxel in the delineated tumour region. Tixier et al. [16] studied the reliability of a number of basic and textural FDG-PET features in a test-retest setting in oesophageal cancer. Although the results presented in that study are not directly comparable to our test-retest results, it can be observed that textural features based on grey-level size-zone matrix representations appear to be the least stable ones, which is also supported by our test-retest, inter-observer and integrated analysis.

While the ICC is a useful tool in assessing the reliability of feature measurements, it is not directly related to a feature's clinical usefulness. For a more complete picture, one would like to know if the inter patient variability or respectively the change in feature values between a reference time point (e.g. pre-treatment) and a point of interest (e.g. during or post treatment) is large enough to be considered useful. To assess this aspect of feature variability, a measure besides the ICC is necessary that provides information on the variability in terms of the feature's unit of measurement. In our study we therefore estimated both the test-retest and inter-observer coefficient of variability for every feature and normalized them to a percentage of the mean feature value as well as the range, to provide easy to interpret values regarding the magnitude of the COV. The larger the COV is compared to inter patient variability or changes in feature values, the less likely it is that the feature under consideration is a useful predictor or biomarker. One has to note however, that like the ICC, COV values are sample specific estimates and typical feature values (i.e. mean and range) are likely to be different when considering different patient populations. Furthermore, the level of variation of a feature that is considered acceptable depends on its intended purpose. 
A limitation of our study is the small number of patients in both cohorts. Although a broad range of tumour sizes and levels of tracer uptake were included, external validation is needed to assess if our results are representative for NSCLC patients in general. Besides feature variability due to repeated imaging and inconsistency between multiple manual tumour delineations, there are more sources of variability that can be taken into consideration. Galavis et al. [17] pointed out that quantitative imaging features are also subject to vary due to different acquisition modes and reconstruction parameters. Also the level of image discretization has been shown to impact the variability of certain textural features, as demonstrated by Tixier et at. [16]. Taking these sources of variability into account, it is evident that standardization is desirable with the prospect of FDGPET Radiomics features for treatment monitoring, outcome prediction or imaging biomarkers.

\section{CONCLUSION}

The aim of this study was to perform an integrated stability analysis of PET Radiomics features obtained from FDG-PET imaging in NSCLC. Our results showed that the majority of assessed features had both a high test-retest (71\%) as well as inter-observer stability $(91 \%)$ in terms of their intra-class correlation coefficient. Furthermore, it was observed that features more stable in repeated PET imaging were in general also more robust against inter-observer variability. Results suggest that further research of quantitative imaging features is warranted with respect to more advanced applications of PET imaging as being used for treatment monitoring, outcome prediction or imaging biomarkers.

\section{ACKNOWLEDGEMENTS}

Authors acknowledge the QuIC-ConCePT project, which is partly funded by EFPIA companies and the Innovative Medicine Initiative Joint Undertaking (IMI JU) under Grant Agreement No. 115151. Authors also acknowledge financial support from the National Institute of Health (NIH-USA U01 CA 143062-01, Radiomics of NSCLC), the CTMM framework (AIRFORCE project, grant 030-103), EU 6th and 7th framework program (EUROXY, METOXIA, EURECA, ARTFORCE), euroCAT (IVA Interreg - www.eurocat.info), Kankeronderzoekfonds Limburg from the Health Foundation Limburg and the Dutch Cancer Society (KWF UM 2011-5020, KWF UM 2009-4454). 


\section{REFERENCES}

[1] Lin P, Koh ES, Lin M, Vinod SK, Ho-Shon I, Yap J, et al. Diagnostic and staging impact of radiotherapy planning FDG-PET-CT in non-small-cell lung cancer. Radiother Oncol 2011;101: 284-290.

[2] De Ruysscher D, Nestle U, Jeraj R, Macmanus M. PET scans in radiotherapy planning of lung cancer. Lung Cancer 2012;75: 141-145.

[3] Van Elmpt W, Pottgen C, De Ruysscher D. Therapy response assessment in radiotherapy of lung cancer. Q J Nucl Med Mol Imaging 2011;55: 648-654.

[4] Thie J. Understanding the standardized uptake value, its methods, and implications for usage. Journal of nuclear medicine 2004;45: 1431-1434.

[5] van Elmpt W, Ollers M, Dingemans AM, Lambin P, De Ruysscher D. Response assessment using 18F-FDG PET early in the course of radiotherapy correlates with survival in advanced-stage non-small cell lung cancer. J Nucl Med 2012;53: 1514-1520.

[6] Takeda A, Yokosuka N, Ohashi T, Kunieda E, Fujii H, Aoki Y, et al. The maximum standardized uptake value (SUVmax) on FDG-PET is a strong predictor of local recurrence for localized non-small-cell lung cancer after stereotactic body radiotherapy (SBRT). Radiother Oncol 2011;101: 291-297.

[7] Velazquez ER, Aerts HJ, Oberije C, De Ruysscher D, Lambin P. Prediction of residual metabolic activity after treatment in NSCLC patients. Acta Oncol 2010;49: 1033-1039.

[8] Vaidya M, Creach KM, Frye J, Dehdashti F, Bradley JD, El Naqa I. Combined PET/CT image characteristics for radiotherapy tumour response in lung cancer. Radiother Oncol 2012;102: 239-245.

[9] Tixier F, Le Rest CC, Hatt M, Albarghach N, Pradier O, Metges JP, et al. Intratumour heterogeneity characterized by textural features on baseline 18F-FDG PET images predicts response to concomitant radiochemotherapy in esophageal cancer. J Nucl Med 2011;52: 369-378.

[10] El Naqa I, Grigsby P, Apte A, Kidd E, Donnelly E, Khullar D, et al. Exploring feature-based approaches in PET images for predicting cancer treatment outcomes. Pattern Recognit 2009;42: 1162-1171.

[11] Buckler AJ, Bresolin L, Dunnick NR, Sullivan DC. Quantitative imaging test approval and biomarker qualification: interrelated but distinct activities. Radiology 2011;259: 875-884.

[12] Lambin P, van Stiphout RG, Starmans MH, Rios-Velazquez E, Nalbantov G, Aerts HJ, et al. Predicting outcomes in radiation oncology--multifactorial decision support systems. Nat Rev Clin Oncol 2013;10: 27-40.

[13] Lambin P, Rios-Velazquez E, Leijenaar R, Carvalho S, van Stiphout RG, Granton P, et al. Radiomics: extracting more information from medical images using advanced feature analysis. Eur J Cancer 2012;48: 441-446.

[14] Kumar V, Gu Y, Basu S, Berglund A, Eschrich SA, Schabath MB, et al. Radiomics: the process and the challenges. Magn Reson Imaging 2012;30: 1234-1248.

[15] de Langen AJ, Vincent A, Velasquez LM, van Tinteren H, Boellaard R, Shankar LK, et al. Repeatability of 18F-FDG uptake measurements in tumours: a metaanalysis. J Nucl Med 2012;53: 701-708.

[16] Tixier F, Hatt M, Le Rest CC, Le Pogam A, Corcos L, Visvikis D. Reproducibility of tumour uptake heterogeneity characterization through textural feature analysis in 18F-FDG PET. J Nucl Med 2012;53: 693-700.

[17] Galavis PE, Hollensen C, Jallow N, Paliwal B, Jeraj R. Variability of textural features in FDG PET images due to different acquisition modes and reconstruction parameters. Acta Oncol 2010;49: 1012-1016.

[18] Frings V, de Langen AJ, Smit EF, van Velden FH, Hoekstra OS, van Tinteren $H$, et al. Repeatability of metabolically active volume measurements with 18F-FDG and 18F-FLT PET in non-small cell lung cancer. J Nucl Med 2010;51: 1870-1877.

[19] Cheebsumon P, Boellaard R, de Ruysscher D, van Elmpt W, van Baardwijk A, Yaqub M, et al. Assessment of tumour size in PET/CT lung cancer studies: PET- and CT-based methods compared to pathology. EJNMMI Res 2012;2: 56.

[20] van Baardwijk A, Bosmans G, Boersma L, Buijsen J, Wanders S, Hochstenbag M, et al. PET-CT-based autocontouring in non-small-cell lung cancer correlates with pathology and reduces interobserver variability 


\section{CHAPTER 5}

in the delineation of the primary tumour and involved nodal volumes. Int J Radiat Oncol Biol Phys 2007;68: 771-778.

[21] Deasy JO, Blanco Al, Clark VH. CERR: a computational environment for radiotherapy research. Med Phys 2003;30: 979-985.

[22] Haralick RM, Shanmugam K, Dinstein I. Textural Features of Image Classification. IEEE T Syst Man Cyb 1973;SMC-3: 610-621.

[23] Galloway M. Texture analysis using gray level run lengths. Comput Vision Graph 1975;4: 172-179.

[24] Shrout PE, Fleiss JL. Intraclass Correlations: Uses in Assessing Rater Reliability. Psychol Bull 1979;86: 420428.

[25] Bland J, Altman D. Agreement between methods of measurement with multiple observations per individual. J Biopharm Stat 2007;17: 571-582. 


\section{DEFINITION OF IMAGING FEATURES}

\section{First-order statistics}

Let $P$ define the first order histogram and $P(i)$ the fraction of voxels with intensity level i. $N_{i}$ is the number of discrete intensity levels.

Energy - the sum of all voxel SUV values squared.

Entropy

$$
\sum_{i=1}^{N_{i}} P(i) \log _{2} P(i)
$$

Kurtosis - the kurtosis of the first order histogram of voxel intensities.

Mean absolute deviation - the mean of the absolute deviations of all voxel intensities around the mean intensity value.

Median - the median intensity value.

Minimum - the minimum intensity value.

$\underline{\text { Range }}$ - the range of intensity values.

Root mean square - the quadratic mean, or the square root of the mean of squares of all voxel intensities.

Skewness - the skewness of the first order histogram of voxel SUV values.

Standard deviation - the standard deviation of all SUV values.

Maximum SUV - the maximum SUV value.

Mean SUV - the mean SUV value.

Peak SUV - defined as the mean SUV within a $1 \mathrm{~cm}^{3}$ sphere centred on the maximum SUV voxel [1].

$\underline{\text { Uniformity }}$

$$
\text { uniformity }=\sum_{i=1}^{N_{l}} P(i)^{2}
$$

Variance - the variance of all SUV values.

\section{Shape and size descriptors}

Geometric features, describing the shape and size of the volume of interest. Let $\mathrm{V}$ be the volume and $\mathrm{A}$ the surface area of the volume of interest.

$\underline{\text { Compactness } 1}$

$$
\frac{V}{\sqrt{\pi} A^{\frac{2}{3}}}
$$

Compactness 2

$$
36 \pi \frac{V^{2}}{A^{3}}
$$

Maximum 3D diameter - the maximum three-dimensional tumour diameter.

Spherical disproportion:

$$
\frac{A}{4 \pi R^{2}}
$$

Where $R$ is the radius of a sphere with the same volume as the tumour.

Sphericity

$$
\frac{\pi^{\frac{1}{3}}(6 V)^{\frac{2}{3}}}{A}
$$

Surface to volume ratio - the surface area divided by the volume. 
3. Gray-Level Co-Occurrence

Matrixes

Gray level co-occurrence matrix based features, as described by Haralick et al

[2].

Let:

$P(i, j)$ be the co-occurrence matrix,

$N_{g}$ be the number of discrete intensity levels in the image, $\mu$ be the mean of $P(i, j)$,

$\mu_{x}(i)$ be the mean of row $i$,

$\mu_{y}(j)$ be the mean of column $j$,

$\sigma_{x}(i)$ be the standard deviation of row $i$,

$\sigma_{y}(j)$ be the standard deviation of column $j$,

$p_{x}(i)=\sum_{j=1}^{N_{g}} P(i, j)$,

$p_{y}(i)=\sum_{i=1}^{N_{g}} P(i, j)$,

$p_{x+y}(k)=\sum_{i=1}^{N_{g}} \sum_{j=1}^{N_{g}} P(i, j)$,

$i+j=k, k=2,3, \ldots, 2 N_{g}$,

$p_{x-y}(k)=\sum_{i=1}^{N_{g}} \sum_{j=1}^{N_{g}} P(i, j)$,

$|i-j|=k, k=0,1, \ldots, N_{g}-1$,

$H X Y 1=$

$-\sum_{i=1}^{N_{g}} \sum_{j=1}^{N_{g}} P(i, j) \log \left(p_{x}(i) p_{y}(j)\right)$,

HXY2

$=-\sum_{i=1}^{N_{g}} \sum_{j=1}^{N_{g}} p_{x}(i) p_{y}(j) \log \left(p_{x}(i) p_{y}(j)\right)$.

Autocorrelation:

$$
\sum_{i=1}^{N_{g}} \sum_{j=1}^{N_{g}} i j P(i, j)
$$

Cluster Prominence

$$
\sum_{i=1}^{N_{g}} \sum_{j=1}^{N_{g}}\left[i+j-\mu_{x}(i)-\mu_{y}(j)\right]^{4} P(i, j)
$$

Cluster Shade

$$
\sum_{i=1}^{N_{g}} \sum_{j=1}^{N_{g}}\left[i+j-\mu_{x}(i)-\mu_{y}(j)\right]^{3} P(i, j)
$$

Cluster Tendency

$$
\sum_{i=1}^{N_{g}} \sum_{j=1}^{N_{g}}\left[i+j-\mu_{x}(i)-\mu_{y}(j)\right]^{2} P(i, j)
$$

Contrast

$$
\sum_{i=1}^{N_{g}} \sum_{j=1}^{N_{g}}|i-j|^{2} P(i, j)
$$

Correlation

$$
\frac{\sum_{i=1}^{N_{g}} \sum_{j=1}^{N_{g}} i j P(i, j)-\mu_{i}(i) \mu_{j}(j)}{\sigma_{x}(i) \sigma_{y}(j)}
$$

Difference entropy

$$
\sum_{i=0}^{N_{g}-1} P_{x-y}(i) \log _{2}\left[P_{x-y}(i)\right]
$$

$\underline{\text { Dissimilarity }}$

$$
\sum_{i=1}^{N_{g}} \sum_{j=1}^{N_{g}}|i-j| P(i, j)
$$

Energy

$$
\sum_{i=1}^{N_{g}} \sum_{j=1}^{N_{g}}[P(i, j)]^{2}
$$

Entropy

$$
-\sum_{i=1}^{N_{g}} \sum_{j=1}^{N_{g}} P(i, j) \log _{2}[P(i, j)]
$$

Homogeneity 1

$$
\sum_{i=1}^{N_{g}} \sum_{j=1}^{N_{g}} \frac{P(i, j)}{1+|i-j|}
$$


Homogeneity 2

$$
\sum_{i=1}^{N_{g}} \sum_{j=1}^{N_{g}} \frac{P(i, j)}{1+|i-j|^{2}}
$$

Informational measure of correlation 1

$$
\frac{H-H X Y 1}{\max \{H X, H Y\}}
$$

Where $\mathrm{H}$ is the entropy.

Informational measure of correlation 2

$$
\sqrt{1-e^{-2(H X Y 2-H)}}
$$

Where $\mathrm{H}$ is the entropy.

$\underline{\text { Inverse Difference Moment Normalized }}$

$$
\sum_{i=1}^{N_{g}} \sum_{j=1}^{N_{g}} \frac{P(i, j)}{1+\left(\frac{|i-j|^{2}}{N^{2}}\right)}
$$

$\underline{\text { Inverse Difference Normalized }}$

$$
\sum_{i=1}^{N_{g}} \sum_{j=1}^{N_{g}} \frac{P(i, j)}{1+\left(\frac{|i-j|}{N}\right)}
$$

Inverse variance

$$
\sum_{i=1}^{N_{g}} \sum_{j=1}^{N_{g}} \frac{P(i, j)}{|i-j|^{2}} \quad, i \neq j
$$

Maximum Probability

$$
\max \{P(i, j)\}
$$

Sum average

$$
\sum_{i=2}^{2 N_{g}}\left[i P_{x+y}(i)\right]
$$

Sum entropy

$$
-\sum_{i=2}^{2 N_{g}} P_{x+y}(i) \log _{2}\left[P_{x+y}(i)\right]
$$

$\underline{\text { Sum variance }}$

$$
\sum_{i=2}^{2 N_{g}}(i-S E)^{2} P_{x+y}(i)
$$

Variance

$$
\sum_{i=1}^{N_{g}} \sum_{j=1}^{N_{g}}(i-\mu)^{2} P(i, j)
$$

\section{Gray-Level Run-Length matrixes}

Gray-level run-length matrix based features, as described by Galloway et al. [3].

Let: $p(i, j \mid \theta)$ be the $(i, j)$ th entry in the given run-length matrix $\mathrm{p}$ for a direction $\theta$,

$\mathrm{N}_{\mathrm{g}}$ the number of discrete intensity values in the image,

$\mathrm{N}_{\mathrm{r}}$ the number of different run lengths, $\mathrm{N}_{\mathrm{p}}$ the number of voxels in the image.

Short Run Emphasis

$$
\frac{\sum_{i=1}^{N_{g}} \sum_{j=1}^{N_{r}}\left[\frac{p(i, j \mid \theta)}{j^{2}}\right]}{\sum_{i=1}^{N_{g}} \sum_{j=1}^{N_{r}} p(i, j \mid \theta)}
$$

Long Run Emphasis

$$
\frac{\sum_{i=1}^{N_{g}} \sum_{j=1}^{N_{r}} j^{2} p(i, j \mid \theta)}{\sum_{i=1}^{N_{g}} \sum_{j=1}^{N_{r}} p(i, j \mid \theta)}
$$

Gray Leven Non-Uniformity

$$
\frac{\sum_{i=1}^{N_{g}}\left[\sum_{j=1}^{N_{r}} p(i, j \mid \theta)\right]^{2}}{\sum_{i=1}^{N_{g}} \sum_{j=1}^{N_{r}} p(i, j \mid \theta)}
$$

Run Length Non-Uniformity

$$
R L N=\frac{\sum_{j=1}^{N_{r}}\left[\sum_{i=1}^{N_{g}} p(i, j \mid \theta)\right]^{2}}{\sum_{i=1}^{N_{g}} \sum_{j=1}^{N_{r}} p(i, j \mid \theta)}
$$

$\underline{\text { Run Percentage }}$

$$
\sum_{i=1}^{N_{g}} \sum_{j=1}^{N_{r}} \frac{p(i, j \mid \theta)}{N_{p}}
$$


Low Gray Level Run Emphasis

$$
\frac{\sum_{i=1}^{N_{g}} \sum_{j=1}^{N_{r}}\left[\frac{p(i, j \mid \theta)}{i^{2}}\right]}{\sum_{i=1}^{N_{g}} \sum_{j=1}^{N_{r}} p(i, j \mid \theta)}
$$

High Gray Level Run Emphasis

$$
\frac{\sum_{i=1}^{N_{g}} \sum_{j=1}^{N_{r}} i^{2} p(i, j \mid \theta)}{\sum_{i=1}^{N_{g}} \sum_{j=1}^{N_{r}} p(i, j \mid \theta)}
$$

Short Run Low Gray Level Emphasis

$$
\frac{\sum_{i=1}^{N_{g}} \sum_{j=1}^{N_{r}}\left[\frac{p(i, j \mid \theta)}{i^{2} j^{2}}\right]}{\sum_{i=1}^{N_{g}} \sum_{j=1}^{N_{r}} p(i, j \mid \theta)}
$$

Short Run High Gray Level Emphasis

$$
\frac{\sum_{i=1}^{N_{g}} \sum_{j=1}^{N_{r}}\left[\frac{p(i, j \mid \theta) i^{2}}{j^{2}}\right]}{\sum_{i=1}^{N_{g}} \sum_{j=1}^{N_{r}} p(i, j \mid \theta)}
$$

Long Run Low Gray Level Emphasis

$$
\frac{\sum_{i=1}^{N_{g}} \sum_{j=1}^{N_{r}}\left[\frac{p(i, j \mid \theta) j^{2}}{i^{2}}\right]}{\sum_{i=1}^{N_{g}} \sum_{j=1}^{N_{r}} p(i, j \mid \theta)}
$$

Long Run High Gray Level Emphasis

$$
\frac{\sum_{i=1}^{N_{g}} \sum_{j=1}^{N_{r}} p(i, j \mid \theta) i^{2} j^{2}}{\sum_{i=1}^{N_{g}} \sum_{j=1}^{N_{r}} p(i, j \mid \theta)}
$$

5. Gray-Level size-zone matrixes

Gray-level size-zone matrix based features, as described by Tixier et al. [4].

Let:

$p(i, j)$ be the $(i, j)$ th entry in the given size-zone matrix $\mathrm{p}$,

$\mathrm{N}_{\mathrm{g}}$ the number of discrete intensity values in the image,

$\mathrm{N}_{\mathrm{z}}$ the size of the largest, homogeneous region in the volume of interest,
$\mathrm{N}_{\mathrm{a}}$ the number homogeneous areas in the image.

Small area Emphasis

$$
\frac{\sum_{i=1}^{N_{g}} \sum_{j=1}^{N_{Z}}\left[\frac{p(i, j)}{j^{2}}\right]}{\sum_{i=1}^{N_{g}} \sum_{j=1}^{N_{Z}} p(i, j)}
$$

Large area Emphasis

$$
\frac{\sum_{i=1}^{N_{g}} \sum_{j=1}^{N_{z}} j^{2} p(i, j)}{\sum_{i=1}^{N_{g}} \sum_{j=1}^{N_{z}} p(i, j)}
$$

$\underline{\text { Intensity variability }}$

$$
\frac{\sum_{i=1}^{N_{g}}\left[\sum_{j=1}^{N_{z}} p(i, j)\right]^{2}}{\sum_{i=1}^{N_{g}} \sum_{j=1}^{N_{z}} p(i, j)}
$$

Size-zone variability

$$
\frac{\sum_{j=1}^{N_{Z}}\left[\sum_{i=1}^{N_{g}} p(i, j)\right]^{2}}{\sum_{i=1}^{N_{g}} \sum_{j=1}^{N_{Z}} p(i, j)}
$$

Zone Percentage

$$
\sum_{i=1}^{N_{g}} \sum_{j=1}^{N_{z}} \frac{p(i, j)}{N_{a}}
$$

Low intensity Emphasis

$$
\frac{\sum_{i=1}^{N_{g}} \sum_{j=1}^{N_{z}}\left[\frac{p(i, j)}{i^{2}}\right]}{\sum_{i=1}^{N_{g}} \sum_{j=1}^{N_{z}} p(i, j)}
$$

High intensity Emphasis

$$
\frac{\sum_{i=1}^{N_{g}} \sum_{j=1}^{N_{z}} i^{2} p(i, j)}{\sum_{i=1}^{N_{g}} \sum_{j=1}^{N_{z}} p(i, j)}
$$

Low intensity small area Emphasis

$$
\frac{\sum_{i=1}^{N_{g}} \sum_{j=1}^{N_{Z}}\left[\frac{p(i, j)}{i^{2} j^{2}}\right]}{\sum_{i=1}^{N_{g}} \sum_{j=1}^{N_{z}} p(i, j)}
$$


High intensity small area Emphasis

$$
\frac{\sum_{i=1}^{N_{g}} \sum_{j=1}^{N_{z}}\left[\frac{p(i, j) i^{2}}{j^{2}}\right]}{\sum_{i=1}^{N_{g}} \sum_{j=1}^{N_{z}} p(i, j)}
$$

Low intensity large area Emphasis

$$
\frac{\sum_{i=1}^{N_{g}} \sum_{j=1}^{N_{Z}}\left[\frac{p(i, j) j^{2}}{i^{2}}\right]}{\sum_{i=1}^{N_{g}} \sum_{j=1}^{N_{z}} p(i, j)}
$$

High intensity large area Emphasis

$$
\frac{\sum_{i=1}^{N_{g}} \sum_{j=1}^{N_{z}} p(i, j) i^{2} j^{2}}{\sum_{i=1}^{N_{g}} \sum_{j=1}^{N_{z}} p(i, j)}
$$




\section{REFERENCES}

[1] Wahl RL, Jacene H, Kasamon Y, Lodge MA. From RECIST to PERCIST: Evolving Considerations for PET response criteria in solid tumours. Journal of nuclear medicine : official publication, Society of Nuclear Medicine. 2009 May;50 Suppl 1:122S-150S.

[2] Haralick RM, Shanmugam K, Dinstein I. Textural Features of Image Classification. IEEE T Syst Man Cyb. 1973;SMC-3(6):610-621.

[3] Galloway M. Texture analysis using gray level run lengths. Comput Vision Graph. 1975;4:172-179.

[4] Tixier F, Hatt M, Le Rest CC, Le Pogam A, Corcos L, Visvikis D. Reproducibility of tumour uptake heterogeneity characterization through textural feature analysis in 18F-FDG PET. J Nucl Med. [Research Support, Non-U.S. Gov't]. 2012 May;53(5):693-700. 


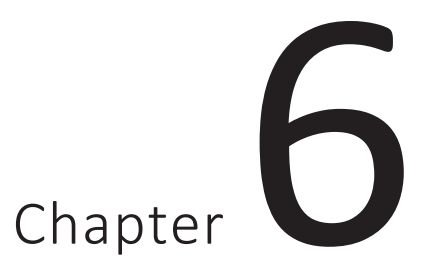

\section{Early variation of FDG-PET Radiomics features in NSCLC is related to overall survival}

\section{The "Delta Radiomics" concept}

Work in preparation

Sara Carvalho, ${ }^{*}$, Ralph T.H. Leijenaar, ${ }^{*}$, Esther G.C. Troost, Janna E. van Timmeren, Wouter van Elmpt, Jean-Pierre Muratet, Fabrice Denis, Tanja Schimek-Jasch, Ursula Nestle, Cary Oberije, Philippe Lambin Early variation of FDG-PET radiomics features in NSCLC is related to overall survival - The "Delta Radiomics" concept

*These authors contributed equally to this work 


\section{ABSTRACT}

Purpose

Early assessment of therapeutic response for non-small cell lung cancer (NSCLC) is of major importance to improve outcome. Radiomics, the analysis of medical imaging, comprises the extraction of descriptors of first order statistics, shape and size, textural information, and intensity volume histograms (IHV). We hypothesized that early changes of metabolic uptake patterns assessed with Radiomics features on repeated 18Ffluorodeoxyglucose positron emission tomography (FDG-PET) during (chemo)radiotherapy are related to overall survival, and evaluated our findings in two independent datasets.

\section{Methods}

Fifty-two stage IIla-IV NSCLC patients, treated with (chemo)radiotherapy, underwent FDG-PET before and after one week of radiotherapy (NCT00522639). A Cox regression prognostic model was fitted to the percentage variation of PET Radiomics features as calculated before and early during radiotherapy - "Delta Radiomics", with a 10-fold cross-validated least absolute shrinkage and selection operator (LASSO) for automatic feature selection. Two independent datasets ( $n=32$ and $n=26)$ were used for performance assessment, calculated with concordance-index. Imaging data of our institute is made publicly available at www.cancerdata.org.

\section{Results}

Derived model included percentage variation of volume (shape), sum entropy (GLCM), high intensity large area emphasis (GLSZM), grey level non-uniformity and long run emphasis (RLGL), and metabolic tumour volume defined for $60 \%$ maximum SUV (IVH), and had a performance of $0.66(p<0.01)$. External validation achieved a performance of $0.64(p<0.01)$ and $0.57(p=0.27)$ in the two independent validation datasets.

\section{Conclusion}

A predictive model was developed and validated in two independent datasets and included percentage variation of radiomics features extracted from repeated FDG-PET scans of the primary tumour in NSCLC patients, reflecting their predictive value and correlation to overall survival. Nevertheless, acquisition protocols should be standardized across institutes, in order to provide with a more satisfactory validation and predictive assessment. 


\section{INTRODUCTION}

Lung cancer is a leading cause of death in cancer patients, with over 1.59 million deaths reported in 2012 by the World Health Organization. There are two distinct forms of the disease: small cell lung cancer (SCLC) and non-small cell lung cancer (NSCLC), with the latter accounting for up to $80 \%$ of the total number of cases. Most patients present with advanced stage disease at diagnosis for which 5 -year survival rates remain of about $15 \%$, which reinforces the need for an early assessment of disease response and effective treatment adaptation, aiming at increasing survival rates $(1,2)$.

Quantitative approaches based on anatomical and functional medical imaging for early response assessment are an emerging field. Computed tomography (CT) is widely used for diagnosis, staging and radiation treatment planning, but fails to accurately assess early tumour response (3). Functional imaging, in particular positron emission tomography (PET), appears to be a more promising tool for tumour response assessment (4-7). Early variation of ${ }^{18} \mathrm{~F}$-fluorodeoxyglucose (FDG) uptake within the tumour volume has been identified as a predictive factor for locally advanced NSCLC, and is commonly explored by standardized uptake value (SUV) descriptors. Changes in commonly used SUV descriptors to assess tumour response, such as the maximum and mean SUV, further extended with metabolic tumour volume (MTV) as well as total lesion glycolysis (TLG, computed by multiplying MTV by the corresponding average SUV), have been reported to be of predictive value (8-13). In addition to these metrics, Radiomics enables the extraction of a large number of quantitative features from FDG-PET images $(14,15)$. Therefore, a more comprehensive characterization of the tumour is expected, beyond the information delivered by the commonly used descriptors. These include first order statistics, shape and size descriptors, textural features, and intensity volume histograms (IVH) (16-18). Given this premise, we hypothesized that early variation of Radiomics metrics derived from repeated FDG-PET acquired before and early during treatment "Delta Radiomics" concept, are potential imaging biomarkers for overall survival assessment. We further conceptualized that these may overcome the predictive potential often associated with most commonly used metrics. We validated our findings in two independent cohorts of NSCLC patients treated with (chemo)radiotherapy. 


\section{MATERIALS AND METHODS}

\section{Study population - Development dataset}

PET scans acquired before and during the second week of radiotherapy were prospectively collected for 52 stage IIIa-IV oligometastatic NSCLC patients referred for radical (chemo)radiotherapy, between September 2010 and March 2012 (NCT00522639). Clinical follow-up was performed according to national guidelines. This study was approved by the Institutional Review Board.

\section{(Chemo)Radiotherapy}

All patients were treated according to a concurrent (chemo)radiotherapy scheme: after 1 cycle of chemotherapy (cisplatin $75-80 \mathrm{mg} / \mathrm{m} 2$ day 1 , etoposide $100 \mathrm{mg} / \mathrm{m} 2$ days $1-3$ ), 45 Gy were delivered twice daily in fractions of 1.5 Gy, followed by maximum of 12 fractions of $2 \mathrm{~Gy}$ (maximum dose $69 \mathrm{~Gy}$ ) once daily, until normal tissue dose constraints was reached (e.g., remaining lung tissue, mediastinal envelope, spinal cord). Cisplatinvinorelbine chemotherapy was administered concurrently on days 2, 9, 23, and 30 (cisplatin $50 \mathrm{mg} / \mathrm{m} 2$ days 2 and 9, $40 \mathrm{mg} / \mathrm{m} 2$ day 23; vinorelbine $20 \mathrm{mg} / \mathrm{m} 2$ day 2 and 9, 15 $\mathrm{mg} / \mathrm{m} 2$ days 23 and 30). Standard dose-reduction rules were applied and cisplatin was substituted by carboplatin in case of renal impairment. The XiO/Focal system (Computerized Medical Systems, St. Louis, MO), based on a convolution-superposition algorithm with inhomogeneity corrections was used for the radiotherapy planning on an FDG-PETCT scan (19-21).

\section{Image acquisition}

Patients underwent an FDG-PET-CT scan before radiotherapy for treatment planning purposes and during the second week of treatment (Figure 1). Before scanning, patients fasted for a minimum of 6 hours. The total administered dose of FDG was calculated as (bodyweight $(\mathrm{Kg}) \times 4+20$ ) MBq. FDG-PET-CT images were acquired 60-minutes post injection on a Siemens Truepoint 40 CT-PET (Siemens AG, Munich, Germany). An Ordered Subset Expectation Maximisation 2D 4 iterations 8 subsets (OSEM2D 4i8s) algorithm with a $5 \mathrm{~mm}$ post-reconstruction Gaussian smoothing filter was used for PET image reconstruction. Pixel size was $4.0728 \times 4.0728$, with slice thickness of $3(\mathrm{~mm})$. All PET scans were corrected for attenuation using the mid-ventilation phase of the 4DCT or a 3DCT thorax, case the 4DCT was not of sufficient image quality due to irregular breathing of the patient. Model-based methods were applied for scatter correction, and all scans were corrected for random events and decay. 

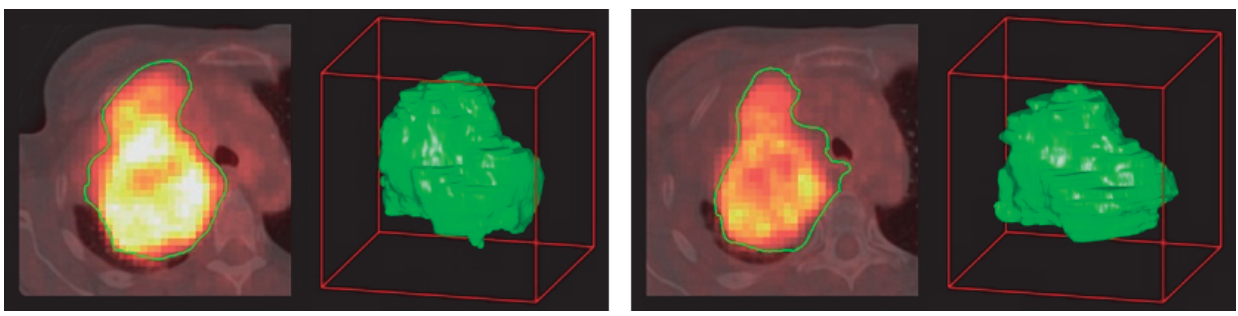

Figure 1 - Example of a pre-treatment (left) and during radiotherapy (right) PET-CT scan of a NSCLC patients from the development dataset. During treatment, and before a volume shrinkage becomes evident, metabolic activity of the tumour can be assessed with a PET tracer, which variation and correlation to outcome can be assessed using Radiomics metrics.

\section{Study population - Validation datasets}

Fifty-eight patients were distributed across two independent validation datasets. Validation dataset 1 comprised 32 stage IIb-IIIb NSCLC and details of the population are published by van Elmpt, et al. (8). Validation dataset 2 included 26 stage II-IIIb NSCLC patients as described by Yossi, et al. (9).

\section{Tumour segmentation and image analysis}

FDG-PET-CT images were imported into treatment planning system using the DICOM protocol. Primary gross tumour volume (GTV) was delineated by experienced radiation oncologists on pre- and during-radiotherapy fused FDG-PET-CT images using appropriate WW-WL settings $(22,23)$. FDG-PET images were converted into standard uptake value (SUV) prior to analysis (24). Image analysis included the investigation of dissimilar group of features, namely descriptors of shape and size, first order statistics, textural information (second order statistics, namely run-length grey level (RLGL), grey level cooccurrence (GLCM) and grey level size zone matrices (GLSZM)) and intensity volume histograms (IVH). To determine textural features, images were first discretized with a bin width of 0.5 (SUV), according to:

$$
I_{D}(x)=\left\lceil\frac{I(x)}{0,5}\right\rceil-\min \left(\left\lceil\frac{I(x)}{0,5}\right\rceil\right)+1
$$

Where I is the original image, I(x) represents the SUV of voxel $x$, and ID is the resulting discretized image (25). Texture features describe spatial distribution of voxel intensities and were determined by considering 26 connected voxels (i.e. voxels were considered to be neighbours in all 13 directions in three dimensions) at a distance of 1 voxel. Features derived from GLCM and RLGL were calculated by averaging their value over all 13 directions. IVHs are similar in concept to dose-volume histograms and describe, in both absolute and relative terms, volume with respect to intensity and vice versa (18). IVH 
also allows retrieving the metabolic tumour volume (MTV) based on isocontour thresholds of maximum intensity within the lesion (from 10 to 90\% (MTV10\% - MTV90\%)) (26), and TLG, by multiplying MTV by the corresponding mean SUV within the segmented volume: TLG10\% - TLG90\% (26, 27). In total, 118 imaging features were calculated based on the FDG-PET distribution within ROI $(17,18)$.

Image analysis was performed in Matlab R2014a (The Mathworks, Natick, MA) using an in-house developed image analysis software used for feature extraction.

Imaging features were analysed based on their percentage variation between subsequent scans, the so-called "Delta Radiomics" approach, and defined as:

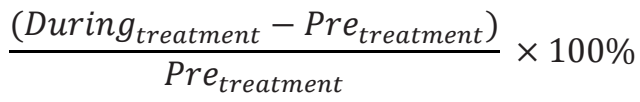

\section{Study parameters/endpoints}

The primary endpoint of this study was overall survival (OS) recorded from start of radiotherapy until the last day of follow-up or death. A patient still alive at the end of the study is considered right-censored. Response to treatment was defined as a minimum decrease of $30 \%$ of maximum SUV on FDG-PET, according to PERCIST criteria, and used to stratify patients (28).

\section{Statistical analysis and model development}

A prior selection of stable and robust features was performed, retaining solely features with a high intraclass correlation (ICC) on both test-retest and inter-observer stability analysis, as described in (17). Median follow-up times were evaluated through a reverse censoring and Kaplan-Meier fit (29).

All feature values were analysed as continuous variables. Wilcoxon rank-sum test was conducted to compare feature values between the non-matched groups of responders and non-responders, based on the PERCIST criteria. Cox Proportional Hazard Regression was performed for overall survival analysis. This was conducted in a univariable approach for the percentage variation of most commonly assessed metrics: volume, maximum SUV, mean SUV, peak SUV, MTV50\% and TLG50\%. Isocontour threshold for MTV and TLG definition was based on literature reported evidences of its appropriateness (10-12).

For model development, a least absolute shrinkage and selection operator (LASSO) method was applied for feature selection, using a 10-fold cross validated intermediate procedure for parameters tuning (30). Regression coefficients were estimated, using the whole development dataset, and hazard ratio's (HR) and confidence intervals were calculated. Model performance was evaluated using Harrell's concordance-index (cindex), for which 1 indicates perfect discrimination and a value of 0.5 no discrimination 
(no greater than the chance expectation), and was reported both internally as externally, using the two independent validation datasets. The log-linearity assumption was verified for the selected features in the final models by fitting a penalized smoothing spline. Features selected for the multivariable model were fitted according to the visual inspection of the linear intervals. The Cox proportional hazards assumption was graphically inspected on Schoenfeld residuals.

All statistical methods were performed in $\mathrm{R}$ (version 2.15.2), using the libraries survcomp, survival, glmnet and corrplot (31).

\section{RESULTS}

Demographic and clinical information from both development and validation datasets is displayed in Table 1. Median follow-up was 3.92 years (95\% Cl: $3.70-4.12$ years), 6.86 years ( $95 \% \mathrm{Cl}: 6.64-6.92$ years) and 4.04 years (95\% Cl: $3.39-\mathrm{NA}$ years) for the development and validation datasets 1 and 2 , respectively.

Table 1 - Clinical data of patients under analysis in the development and validation datasets.

\begin{tabular}{|c|c|c|c|}
\hline & Development $(n=52)$ & Validation $1(n=32)$ & Validation $2(n=26)$ \\
\hline \multicolumn{4}{|l|}{ Age [years] } \\
\hline Range (median) & $35-86$ & $46-82(64)$ & $41-76(62)$ \\
\hline Mean \pm SD & $62 \pm 10$ & $65 \pm 9$ & $61 \pm 8$ \\
\hline \multicolumn{4}{|l|}{ Gender } \\
\hline Male & $29(56 \%)$ & $23(72 \%)$ & $24(92 \%)$ \\
\hline Female & $23(44 \%)$ & $9(28 \%)$ & $2(8 \%)$ \\
\hline \multicolumn{4}{|l|}{ Stage } \\
\hline$\|$ & - & $2(6 \%)$ & $4(15 \%)$ \\
\hline IIla & $18(35 \%)$ & $14(44 \%)$ & $15 *(58 \%)$ \\
\hline$\| l l b$ & $26(50 \%)$ & $16(50 \%)$ & $7(27 \%)$ \\
\hline IV & $8(15 \%)$ & - & - \\
\hline \multicolumn{4}{|l|}{ Histology } \\
\hline Adenocarcinoma & $17(32 \%)$ & $6(19 \%)$ & $11(42 \%)$ \\
\hline Squamous cell carcinoma & $14(27 \%)$ & $9(28 \%)$ & $13(50 \%)$ \\
\hline NSCLC Otherwise Specified & $19(37 \%)$ & $17(53 \%)$ & $2(8 \%)$ \\
\hline Unknown & $2(4 \%)$ & - & - \\
\hline \multicolumn{4}{|l|}{ Radiotherapy (dose) } \\
\hline Range (median) & $45-95(69)$ & $46-70(61)$ & $66-70(66)$ \\
\hline Mean \pm SD & $66 \pm 7$ & $61 \pm 7$ & $68 \pm 2$ \\
\hline
\end{tabular}




\begin{tabular}{lccc}
\hline & Development $(\mathrm{n}=52)$ & Validation $1(\mathrm{n}=32)$ & Validation 2 ( $\mathrm{n}=26)$ \\
\hline Chemotherapy & & & \\
Concurrent & $52(100 \%)$ & $13(41 \%)$ & $26(100 \%)$ \\
Sequential & - & $18(56 \%)$ & - \\
No & - & $1(3 \%)$ & $5-93(33)$ \\
Interval PET - First RT (days) & & $2-13(8)$ & $40 \pm 21$ \\
Range (median) & $7-16(7)$ & $8 \pm 2$ & $15-32(22)$ \\
Mean \pm SD & $7 \pm 2$ & $6-19(8)$ & $21 \pm 4$ \\
Interval First RT - PET (days) & $15 \pm 2$ & $9 \pm 3$ & \\
Range (median) & & & $21-110(54)$ \\
Mean \pm SD & $17-27(22)$ & $10-24(16)$ & $61 \pm 21$ \\
Interval between PET scans (days) & $22 \pm 2$ & $17 \pm 3$ & \\
Range (median) & & & \\
Mean \pm SD & &
\end{tabular}

* Includes one TXN2M0 patient, for which the merged structure between node and tumour was analysed.

** Solely one patient was acquired with an interval over 30 days after start of radiotherapy.

Identification of stable and robust Radiomics features resulted in the selection of 77 features for analysis. Response to treatment based on a $30 \%$ decrease of maximum SUV, as in the PERCIST criteria, identified a group of 24 responders (maximum SUV variation from $-30 \%$ to $-79 \%[-46 \pm 13]$ ) and 28 non-responders (maximum SUV variation

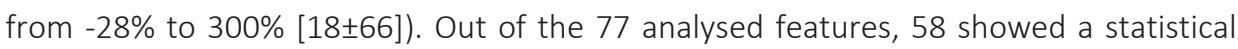
mean feature value difference between responders and non-responders. None of the shape and size metrics showed evidence for early response differentiation, along with IVH metrics related to absolute tumour volume portions with increasing intensity (MTV) in the development cohort. On the other hand, first order statistics metrics, including maximum, peak and mean SUV, were all statistically related to outcome. Both TLG descriptors derived from IVH metrics, as well as all investigated texture metrics, with the exception of grey level non-uniformity, from the RLGL sub-category could be associated with early response discrimination as well. 
Table 2 - Cox regression on the percentage variation of the PET imaging descriptors most commonly used, reporting the univariable hazard ratio (HR), 95\% confidence interval ( $\mathrm{Cl}$ ) of the HR and corresponding $\mathrm{p}$-value. Univariable performance is reported in terms of the concordance-index (c-index). Pre and during radiotherapy (RT) absolute values, and percentage variation between PET acquisitions of the analysed metrics is also presented (mean \pm standard deviation).

\begin{tabular}{lccccccc}
\hline & Pre-RT & During RT & $\begin{array}{c}\text { Percentage } \\
\text { variation }\end{array}$ & HR & $95 \% \mathrm{Cl}$ & p-value & c-index \\
\hline Volume $\left[\mathrm{cm}^{3}\right]$ & $79 \pm 110$ & $68 \pm 108$ & $-21 \pm 41$ & 1.00 & $0.99-1.01$ & 0.46 & 0.53 \\
Maximum SUV & $11 \pm 6.0$ & $8.3 \pm 4.0$ & $-11 \pm 59$ & 1.00 & $1.00-1.01$ & 0.90 & 0.51 \\
Mean SUV & $4.7 \pm 2.4$ & $3.5 \pm 1.5$ & $-0.4 \pm 112$ & 1.00 & $1.00-1.00$ & 0.80 & 0.53 \\
Peak SUV & $8.7 \pm 5.1$ & $6.3 \pm 3.3$ & $-14 \pm 56$ & 1.00 & $0.99-1.01$ & 0.98 & 0.50 \\
MTV $_{50 \%}$ & $22 \pm 34$ & $17 \pm 27$ & $-18 \pm 50$ & 1.00 & $0.99-1.01$ & 0.63 & 0.51 \\
TLG $_{50 \%}$ & $198 \pm 352$ & $105 \pm 180$ & $-35 \pm 50$ & 1.00 & $1.00-1.00$ & 0.74 & 0.50 \\
\hline
\end{tabular}

Acronyms: RT - Radiotherapy; HR - hazard ratio; Cl - confidence interval; c-index - concordance-index; SUV Standard uptake value; MTV $_{50 \%}$ - Metabolic Tumour Volume defined for an isocontour of 50\% maximum SUV (assessed from absolute volume of relative intensity, from IVH metrics); $\mathrm{TLG}_{50 \%}$ - Total Lesion Glycolysis defined for an isocontour of $50 \%$ maximum SUV.

Univariable analysis of percentage variation of most common metabolic metrics in the development cohort is presented in Table 2. From this analysis one can appreciate nonsignificant hazard ratios (HR) of early percentage variation of volume ( $H R=0.99, p=0.46)$, maximum SUV ( $H R=1.00, p=0.90)$, peak SUV ( $H R=1.00, p=0.98)$, mean SUV ( $H R=1.00$, $p=0.80), M T V 50 \%(H R=1.00, p=0.63)$, and TLG50\% ( $H R=1.00, p=0.74)$. A multivariable fit of the data with a LASSO feature selection methodology returned a model including the percentage variation of gross tumour volume (shape), textural information described from grey level non-uniformity and long run emphasis (RLGL) sum entropy (GLCM), and high intensity large area emphasis (GLSZM), and MTV derived from the absolute volume of a relative intensity of $60 \%$ - MTV60\% (IVH), which detail can be gathered in Table 3. Pre and during radiotherapy feature values, as well as percentage variation of these are shown in Table 4, for the three datasets analysed. None of the features from the first order statistics sub-category nor TLG were selected for the model.

Features not verifying the log-linearity assumption, were entered in the derived model by means of a piecewise linear approach, which intervals were verified visually on the fitted smoothing splines (online appendix). All features met the proportional hazards assumption. After these corrections, internal performance of the model in the derivation dataset reached a c-index of $0.66(p<0.01)$. External validation had a performance of 0.64 $(p<0.01)$ for validation dataset 1 and $0.57(p=0.27)$ for validation dataset 2 . 


\section{CHAPTER 6}

Table 3 - Developed model after LASSO feature selection on the training dataset. Hazard ratios (HR), corresponding 95\% confidence interval $(\mathrm{Cl})$ and p-value are presented for the final model. Performance is expressed as the concordance-index ( $c$-index) both internally as for the two independent validation datasets ( $p$ value between brackets).

\begin{tabular}{|c|c|c|c|c|c|c|}
\hline \multirow[t]{2}{*}{ Features in the model } & \multirow[t]{2}{*}{$H R$} & \multirow[t]{2}{*}{$95 \% \mathrm{Cl}$} & \multirow[t]{2}{*}{ p-value } & \multirow[t]{2}{*}{ c-index } & \multicolumn{2}{|c|}{ External Validation } \\
\hline & & & & & Dataset 1 & Dataset 2 \\
\hline \multicolumn{7}{|l|}{ Shape-Volume } \\
\hline$<50 \%$ & 0.98 & $0.96-1.01$ & 0.29 & & & \\
\hline$\geq 50 \%$ & 0.88 & $0-1.22 \mathrm{e}^{34}$ & 0.99 & & & \\
\hline \multicolumn{7}{|l|}{ RLGL - Grey level non-uniformity } \\
\hline$<150 \%$ & 1.02 & $1.00-1.03$ & 0.07 & & & \\
\hline$\geq 150 \%$ & 1.01 & $1.00-1.02$ & 0.19 & & & \\
\hline RLGL - Long run emphasis & 0.97 & $0.94-1.00$ & 0.06 & 0.66 & 0.64 & 0.57 \\
\hline GLCM - Sum entropy & & & & $(<0.01)$ & $(<0.01)$ & $(0.27)$ \\
\hline$<0 \%$ & 0.97 & $0.93-1.02$ & 0.30 & & & \\
\hline$\geq 0 \%$ & 0.99 & $0.96-1.02$ & 0.59 & & & \\
\hline $\begin{array}{l}\text { GLSZM - High intensity large area } \\
\text { emphasis }\end{array}$ & 1.00 & $1.00-1.00$ & 0.41 & & & \\
\hline \multicolumn{7}{|l|}{$\mathrm{IVH}-\mathrm{MTV}_{60 \%}$} \\
\hline$<40 \%$ & 1.00 & $0.99-1.02$ & 0.76 & & & \\
\hline$\geq 40 \%$ & 1.00 & $0.98-1.01$ & 0.42 & & & \\
\hline
\end{tabular}

Acronyms: RLGL - run-length grey level; GLCM - grey level co-occurrence; GLSZM - grey level size zone matrices; IVH - intensity-volume histograms; AVRI - absolute volume of a relative intensity; $\mathrm{HR}$ - hazard ratio; $\mathrm{Cl}$ confidence interval; c-index - concordance-index 


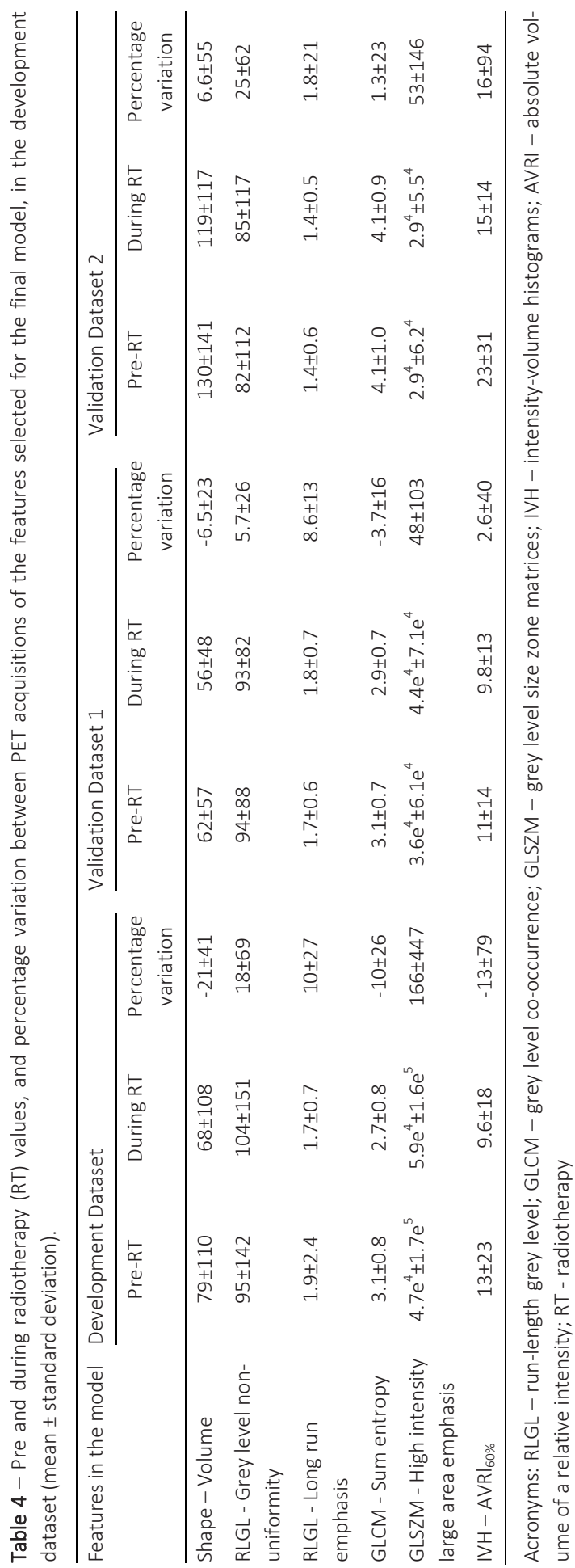




\section{DISCUSSION}

The need for a fast and convenient method of early assessment of the treatment efficacy and response has gained more attention, especially with regard to fulfil the promise of personalized and adapted treatment (32). A large clinical impact is expected as therapy can still be adapted early into treatment, for instance by radiation dose escalation protocols or additional systemic treatment. Functional imaging appears to be a more valuable tool than anatomical imaging, due to its ability to visualize changes in metabolic activity of the tumour before volume shrinkage becomes evident. A full Radiomics approach is expected to provide a better characterization of this metabolic information by encompassing first order statistics, shape and size descriptors, textural features and IVH descriptors, and therefore not limited to the commonly assessed metrics based on the SUV patterns of the primary tumour, as maximum, peak and mean SUV (16-18). In this study we analysed and derived a prognostic multivariable model based on the percentage variation of Radiomics features of FDG-PET scans acquired before and early during treatment and further validated these findings in two independent datasets.

In 2009, PERCIST criteria introduced a $30 \%$ or greater SUV decline as being associated with response to treatment, for which relevant beneficial changes in the course of treatment could still be adopted (28). Radiomics features related to first order statistics, texture description of the tumour and IVH characterization showed here a good relation to response classification based on this criterion. Neither shape and size metrics nor the IVH related to absolute volume defined for different relative intensity thresholds (i.e., metabolic tumour volume) could provide discrimination between groups. These observations are aligned with the expectation that volume variation early during treatment does not provide an insight on how patients respond, while metabolic activity does.

The reported dependence between FDG distribution and tumour's microenvironment turns that into a valuable tool for non-invasive tumour's behaviour assessment (33). Intrinsic heterogeneous distribution of FDG within tumour volume has been correlated with several and distinct factors such as necrosis (34), cellular proliferation (35), and hypoxia (36). This represents reasoning to derive more complex descriptors of FDG distribution beyond maximum, peak, or mean uptake. Maximum and peak SUV reflect variations in a single pixel/region and may often suffer from a random interference of noise rather than an actual abnormal uptake. SUV mean cannot describe a more complex representation of heterogeneous FDG distribution within the tumour, reflecting solely global changes in SUV. Univariable analysis of early variation of these metrics, showed no correlation to outcome in the development dataset, despite their reported importance in a prognostic setting $(37,38)$. These preliminary observations strengthen the expected gain in performing a "Delta-Radiomics" analysis that would provide a better identification of diversified response areas within tumour volume, and therefore to have a great value for response monitoring $(39,40)$. The derived predictive model included the percentage variation of at least one feature from each sub-category, except 
from first order statistics, and had an internal performance of 0.66 . Selected features included percentage variation of volume, which prognostic value of pre-treatment value has already been reported (41). Being an anatomic metric, its predictive power due to early variations during treatment may not be an obvious feature, however, in our study we did find its percentage decrease to be associated with overall survival, in the scope of the multivariable combination, despite its pre-treatment value not showing statistical significant correlation to outcome. In addition, variation of metabolic tumour volume defined for an isocontour of 60\% maximum SUV (MTV60\%), which has already received recognition to be a prognostic factor for NSCLC, was also added to the multivariable model $(42,43)$. Previous evidences have shown that textural parameters as extracted from the primary tumour prior to (chemo)radiotherapy delivery are associated with response to treatment and survival $(44,45)$. These are representative of the FDG distribution heterogeneity across the whole tumour, and at least one feature of each subcategory amongst texture descriptors was selected for the derived model.

Discrimination is an essential tool identifying whether patients with outcome have higher risk predictions than those without. In the development phase, one is interested in a model that fits validly in the development dataset, but also independently in nonrelated yet similar datasets, as to extend the derived predictions into a broader population. In the current study, we validated our findings in two independent datasets, and reached performances of 0.64 and 0.57 , respectively. Commonly a reduced accuracy tends to be observed when validating the model against new, independent, and external patient's cohorts. Most common reasons is a reflection of major dissimilarities between derivation and validation data (46). In the external validation we noticed a drop in the performance for the two independent validation datasets, in comparison to the derivation dataset. Reasons for this reduced performance can have several explanations as different timing and delivered dose at time of the second scan, or chemotherapy schemes were followed. This particular last item could potentially affect the validation performance on first dataset, as all patients in the development dataset followed a concurrent scheme, expected to diminish the proliferation potential of the tumour, and therefore be associated with a better prognosis $(47,48)$. Acquisition protocols should also be standardized in order to provide a better validation of our results. This particularly is reflected in the second validation scan that inherently suffers from a great deviation in comparison to development and first validation dataset, and presents with longer intervals between first scan and treatment initiation. This was also patent in the dissimilar patterns of features variation and possibly plays a role in the lower validation accuracy achieved.

In the future we would also like to extend our analysis to other involved structures in the NSCLC, namely the mediastinal and hilar lymph nodes. A recent study reported on the added value of analysing the percentage variation of the maximum SUV of the FDGavid lymph nodes in comparison to as extracted from the primary tumour (49). We would like to extend the here presented Delta-Radiomics approach to other endpoints, 
in addition to overall survival, in order to provide with a better and more informative model. This was, however, not possible to perform at the time of analysis.

\section{CONCLUSION}

We developed and validated in two independent datasets a Delta-radiomics predictive model, presenting a satisfactory predictive accuracy for disease's progression and patient's performance. Delta-Radiomics features also appear to be promising descriptors for early response assessment. Nevertheless, acquisition protocols should be standardized across institutes, in order to provide with a more satisfactory validation and predictive assessment.

\section{ACKNOWLEDGEMENTS}

The authors acknowledge financial support from the QulC-ConCePT project, which is partly funded by EFPIA companies and the Innovative Medicine Initiative Joint Undertaking (IMIJU) under Grant Agreement No. 115151. The authors also acknowledge financial support from the National Institute of Health (NIH-USA U01 CA 143062-01, Radiomics of NSCLC), the CTMM framework (AIRFORCE project, grant 030-103), EU 6th and 7th framework program (METOXIA, EURECA, ARTFORCE), euroCAT (IVA Interreg www.eurocat.info), Kankeronderzoekfonds Limburg from the Health Foundation Limburg and the Dutch Cancer Society (KWF UM 2008-4210, KWF UM 2009-4454). 


\section{REFERENCES}

[1] Travis WD BE, Müller-Hermelink HK, Harris CC. Classification of Tumours - Pathology and Genetics of Tumours of the Lung, Pleura,Thymus and Heart. Lyon, France: IARC Press, International Agency for Research on Cancer; 2004.

[2] Crino L, Weder W, van Meerbeeck J, Felip E. Early stage and locally advanced (non-metastatic) nonsmall-cell lung cancer: ESMO Clinical Practice Guidelines for diagnosis, treatment and follow-up. Ann Oncol. 2010;21 Suppl 5:v103-115.

[3] Brindle K. New approaches for imaging tumour responses to treatment. Nat Rev Cancer. 2008;8:94-107.

[4] Decoster L, Schallier D, Everaert H, et al. Complete metabolic tumour response, assessed by 18fluorodeoxyglucose positron emission tomography (18FDG-PET), after induction chemotherapy predicts a favourable outcome in patients with locally advanced non-small cell lung cancer (NSCLC). Lung Cancer. 2008;62:55-61.

[5] Storto G, Nicolai E, Salvatore M. [18F]FDG-PET-CT for early monitoring of tumour response: when and why. Q J Nucl Med Mol Imaging. 2009;53:167-180.

[6] Van Elmpt W, Pottgen C, De Ruysscher D. Therapy response assessment in radiotherapy of lung cancer. Q J Nucl Med Mol Imaging. 2011;55:648-654.

[7] Grootjans W, de Geus-Oei LF, Troost EG, Visser EP, Oyen WJ, Bussink J. PET in the management of locally advanced and metastatic NSCLC. Nat Rev Clin Oncol. 2015;12:395-407.

[8] van Elmpt W, Ollers M, Dingemans AM, Lambin P, De Ruysscher D. Response assessment using 18F-FDG PET early in the course of radiotherapy correlates with survival in advanced-stage non-small cell lung cancer. J Nucl Med. 2012;53:1514-1520.

[9] Yossi S, Krhili S, Muratet JP, Septans AL, Campion L, Denis F. Early assessment of metabolic response by 18F-FDG PET during concomitant radiochemotherapy of non-small cell lung carcinoma is associated with survival: a retrospective single-center study. Clin Nucl Med. 2015;40:e215-221.

[10] Usmanij EA, de Geus-Oei LF, Troost EG, et al. 18F-FDG PET early response evaluation of locally advanced non-small cell lung cancer treated with concomitant chemoradiotherapy. J Nucl Med. 2013;54:15281534.

[11] Huang W, Zhou T, Ma L, et al. Standard uptake value and metabolic tumour volume of (1)(8)F-FDG $\mathrm{PET} / \mathrm{CT}$ predict short-term outcome early in the course of chemoradiotherapy in advanced non-small cell lung cancer. Eur J Nucl Med Mol Imaging. 2011;38:1628-1635.

[12] Massaccesi M, Calcagni ML, Spitilli MG, et al. (1)(8)F-FDG PET-CT during chemo-radiotherapy in patients with non-small cell lung cancer: the early metabolic response correlates with the delivered radiation dose. Radiat Oncol. 2012;7:106.

[13] Huang W, Fan M, Liu B, et al. Value of metabolic tumour volume on repeated 18F-FDG PET/CT for early prediction of survival in locally advanced non-small cell lung cancer treated with concurrent chemoradiotherapy. J Nucl Med. 2014;55:1584-1590.

[14] Lambin P, Rios-Velazquez E, Leijenaar R, et al. Radiomics: extracting more information from medical images using advanced feature analysis. Eur J Cancer. 2012;48:441-446.

[15] Gillies RJ, Kinahan PE, Hricak H. Radiomics: Images Are More than Pictures, They Are Data. Radiology. 2015:151169.

[16] Aerts HJ, Velazquez ER, Leijenaar RT, et al. Decoding tumour phenotype by noninvasive imaging using a quantitative radiomics approach. Nat Commun. 2014;5:4006.

[17] Leijenaar RT, Carvalho S, Velazquez ER, et al. Stability of FDG-PET Radiomics features: an integrated analysis of test-retest and inter-observer variability. Acta Oncol. 2013;52:1391-1397.

[18] Carvalho S, Leijenaar RT, Velazquez ER, et al. Prognostic value of metabolic metrics extracted from baseline positron emission tomography images in non-small cell lung cancer. Acta Oncol. 2013;52:13981404.

[19] Oncology MR. Radiotherapy for Stage I-III Non-small Cell Lung Cancer to an Individualized MLD. 2010, July 20; http://clinicaltrials.gov/show/NCT00573040. Accessed January 8, 2014. 
[20] van Baardwijk A, Bosmans G, Boersma L, et al. Individualized radical radiotherapy of non-small-cell lung cancer based on normal tissue dose constraints: a feasibility study. Int J Radiat Oncol Biol Phys. 2008;71:1394-1401.

[21] van Baardwijk A, Bosmans G, Boersma L, et al. PET-CT-based auto-contouring in non-small-cell lung cancer correlates with pathology and reduces interobserver variability in the delineation of the primary tumour and involved nodal volumes. Int J Radiat Oncol Biol Phys. 2007;68:771-778.

[22] De Ruysscher D. PET-CT in radiotherapy for lung cancer. Methods Mol Biol. 2011;727:53-58.

[23] De Ruysscher D, Nestle U, Jeraj R, Macmanus M. PET scans in radiotherapy planning of lung cancer. Lung Cancer. 2012;75:141-145.

[24] Kinahan PE, Fletcher JW. Positron emission tomography-computed tomography standardized uptake values in clinical practice and assessing response to therapy. Semin Ultrasound CT MR. 2010;31:496-505.

[25] Leijenaar RT, Nalbantov G, Carvalho S, et al. The effect of SUV discretization in quantitative FDG-PET Radiomics: the need for standardized methodology in tumour texture analysis. Sci Rep. 2015;5:11075.

[26] Van de Wiele C, Kruse V, Smeets P, Sathekge M, Maes A. Predictive and prognostic value of metabolic tumour volume and total lesion glycolysis in solid tumours. Eur J Nucl Med Mol Imaging. 2013;40:290-301.

[27] Visser EP, Philippens ME, Kienhorst L, et al. Comparison of tumour volumes derived from glucose metabolic rate maps and SUV maps in dynamic 18F-FDG PET. J Nucl Med. 2008;49:892-898.

[28] Wahl RL, Jacene H, Kasamon Y, Lodge MA. From RECIST to PERCIST: Evolving Considerations for PET response criteria in solid tumours. J Nucl Med. 2009;50 Suppl 1:122S-150S.

[29] Schemper M, Smith TL. A note on quantifying follow-up in studies of failure time. Control Clin Trials. 1996;17:343-346.

[30] Tibshirani R. The lasso method for variable selection in the Cox model. Stat Med. 1997;16(4):385-395.

[31] R: A language and environment for statistical computing [computer program]. Version 2.15.2; 2015.

[32] Lambin P, Roelofs E, Reymen B, et al. 'Rapid Learning health care in oncology' - An approach towards decision support systems enabling customised radiotherapy'. Radiother Oncol. 2013.

[33] Pugachev A, Ruan S, Carlin S, et al. Dependence of FDG uptake on tumour microenvironment. Int J Radiat Oncol Biol Phys. 2005;62:545-553.

[34] Sorensen M, Horsman MR, Cumming P, Munk OL, Keiding S. Effect of intratumoural heterogeneity in oxygenation status on FMISO PET, autoradiography, and electrode Po2 measurements in murine tumours. Int J Radiat Oncol Biol Phys. 2005;62:854-861.

[35] Avril N, Menzel M, Dose J, et al. Glucose metabolism of breast cancer assessed by 18F-FDG PET: histologic and immunohistochemical tissue analysis. J Nucl Med. 2001;42:9-16.

[36] Troost EG, Bussink J, Kaanders JH, et al. Comparison of different methods of CAIX quantification in relation to hypoxia in three human head and neck tumour lines. Radiother Oncol. 2005;76:194-199.

[37] Paesmans M, Garcia C, Wong CY, et al. Primary tumour standardised uptake value is prognostic in nonsmall cell lung cancer: a multivariate pooled analysis of individual data. Eur Respir J. 2015;46:1751-1761.

[38] Fried DV, Mawlawi O, Zhang L, et al. Stage III Non-Small Cell Lung Cancer: Prognostic Value of FDG PET Quantitative Imaging Features Combined with Clinical Prognostic Factors. Radiology. 2016;278:214-222.

[39] El Naqa I, Grigsby P, Apte A, et al. Exploring feature-based approaches in PET images for predicting cancer treatment outcomes. Pattern Recognit. 2009;42:1162-1171.

[40] van Velden FH, Cheebsumon P, Yaqub M, et al. Evaluation of a cumulative SUV-volume histogram method for parameterizing heterogeneous intratumoural FDG uptake in non-small cell lung cancer PET studies. Eur J Nucl Med Mol Imaging. 2011;38:1636-1647.

[41] Oberije C, De Ruysscher D, Houben R, et al. A Validated Prediction Model for Overall Survival From Stage III Non-Small Cell Lung Cancer: Toward Survival Prediction for Individual Patients. Int J Radiat Oncol Biol Phys. 2015;92:935-944.

[42] Ohri N, Duan F, Machtay M, et al. Pretreatment FDG-PET metrics in stage III non-small cell lung cancer: ACRIN 6668/RTOG 0235. J Natl Cancer Inst. 2015;107.

[43] Huang W, Liu B, Fan M, et al. The early predictive value of a decrease of metabolic tumour volume in repeated (18)F-FDG PET/CT for recurrence of locally advanced non-small cell lung cancer with concurrent radiochemotherapy. Eur J Radiol. 2015;84:482-488. 
[44] Cook GJ, Yip C, Siddique M, et al. Are pretreatment 18F-FDG PET tumour textural features in non-small cell lung cancer associated with response and survival after chemoradiotherapy? J Nucl Med. 2013;54:19-26.

[45] Hatt M, Majdoub M, Vallieres M, et al. 18F-FDG PET uptake characterization through texture analysis: investigating the complementary nature of heterogeneity and functional tumour volume in a multicancer site patient cohort. J Nucl Med. 2015;56:38-44.

[46] Toll DB, Janssen KJ, Vergouwe Y, Moons KG. Validation, updating and impact of clinical prediction rules: a review. J Clin Epidemiol. 2008;61:1085-1094.

[47] Ozcelik M, Korkmaz T, Odabas H, et al. Comparison of efficacy and safety of three different chemotherapy regimens delivered with concomitant radiotherapy in inoperable stage III non-small cell lung cancer patients. Tumour Biol. 2016.

[48] Curran WJ, Jr., Paulus R, Langer CJ, et al. Sequential vs. concurrent chemoradiation for stage III non-small cell lung cancer: randomized phase III trial RTOG 9410. J Natl Cancer Inst. 2011;103:1452-1460.

[49] Markovina S, Duan F, Snyder BS, Siegel BA, Machtay M, Bradley JD. Regional Lymph Node Uptake of [(18)F]Fluorodeoxyglucose After Definitive Chemoradiation Therapy Predicts Local-Regional Failure of Locally Advanced Non-Small Cell Lung Cancer: Results of ACRIN 6668/RTOG 0235. 



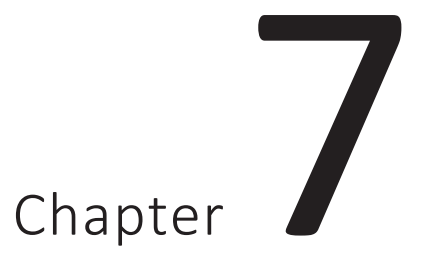

\section{FDG-PET Radiomics of metastatic lymph nodes and primary tumour in NSCLC}

\section{A prospective study externally validated}

FDG-PET Radiomics of metastatic lymph nodes and primary tumour in NSCLC -

A prospective study externally validated

Sara Carvalho, Ralph T.H. Leijenaar, Esther G.C. Troost, Janna E. van Timmeren,

Cary Oberije, Wouter van Elmpt, Lioe-Fee de Geus-Oei, Johan Bussink, Philippe Lambin 


\begin{abstract}
Background

Lymph node stage prior to treatment is strongly related to disease progression and poor prognosis in non-small cell lung cancer (NSCLC). However, few studies have investigated metabolic imaging features derived from pre-radiotherapy 18F-fluorodeoxyglucose (FDG) positron-emission tomography (PET) of metastatic hilar/mediastinal lymph nodes (LNs). We hypothesised that these would provide complementary prognostic information to FDG-PET descriptors of the primary tumour (tumour).
\end{abstract}

\title{
Methods
}

Two independent datasets of 262 and 50 node-positive NSCLC patients were used for model development and validation. Image features (i.e. Radiomics) including shape and size, first order statistics, texture, and intensity-volume histograms (IVH) (www.radiomics.org) were evaluated by univariable Cox regression on the development dataset. Prognostic modelling was conducted with a 10-fold cross-validated least absolute shrinkage and selection operator (LASSO), automatically selecting amongst FDGPET-Radiomics descriptors from (1) tumour, (2) LNs or (3) both structures. Performance was assessed with the concordance-index. Development data are available at www.cancerdata.org.

\section{Results}

Common SUV descriptors (maximum, peak, and mean) were significantly related to overall survival when extracted from LNs, as were LN volume and tumour load (summed volumes of tumour and LN), though this was not true for either SUV metrics or tumour's volume. Feature selection exclusively from imaging information based on FDG-PETRadiomics, exhibited performances of (1) 0.53 - external 0.54, when derived from the tumour, (2) 0.62 - external 0.56 from LNs, and (3) 0.62 - external 0.59 from both structures, including at least one feature from each sub-category, except IVH.

\section{Conclusion}

Combining imaging information based on FDG-PET-Radiomics features from tumours and LNs is desirable to achieve a higher prognostic discriminative power for NSCLC. 


\section{INTRODUCTION}

Non-small cell lung cancer (NSCLC) patients often present with hilar and/or mediastinal lymph node involvement at diagnosis or during the course of disease. Lymph node stage prior to treatment is strongly related to disease progression and worse prognosis [1]. Furthermore, it affects treatment selection and target volume definition, for metastatic lymph nodes in patients eligible for high-dose (chemo)radiotherapy [2]. Apart from the number of metastatic lymph node stations, lymph node size and corresponding metabolic activity may vary among patients [3]. Diagnosis of metastatic hilar/mediastinal lymph nodes is commonly performed through $18 \mathrm{~F}-$ fluorodeoxyglucose positron emission tomography-computed tomography (FDG-PET-CT) and consecutive endoscopic ultrasound bronchoscopy/oesophagoscopy (EBUS/EUS) or mediastinoscopy [4-7].

Metabolic imaging of NSCLC is being explored more and more widely for use in prognostics, based on the patterns of the standard uptake value (SUV) of FDG by the primary tumour, as an extension of its primary diagnostic function: detection of metastatic lymph nodes and distant metastasis $[8,9]$. SUV patterns in FDG-PET can be analysed by means of a Radiomics-based approach, comprising the extraction of a large set of imaging descriptors [10]. The underlying hypothesis based on radiomics is that biomarkers of imaging phenotypes deliver complementary and clinically relevant information, which could be incorporated into individualised radiation oncology approaches and shared decision-making tools [11-14].

In this study we hypothesised that the local selection of more aggressive cancer cells in the metastatic hilar/mediastinal lymph nodes, is more likely to determine prognosis, and therefore provide an additional and valuable source of information to the primary tumour for NSCLC patients. To demonstrate this we performed a combined PETRadiomics analysis of metabolic activity as measured with FDG uptake in both primary tumour and metastatic lymph nodes, and further validated these results in an independent dataset.

\section{PATIENTS AND METHODS}

\section{Development cohort}

\section{Patient population}

The prospective data collection was approved by the Institutional Review Board of our institute (NCT00522639). Electronic medical charts of non-small cell lung cancer (NSCLC) patients were reviewed. Patients undergoing surgery, Stereotactic Body Radiotherapy (SBRT) or palliative treatment, or who had had a previous malignancy within five years prior to diagnosis were excluded from analysis. A total of 343 NSCLC patients (stage I-IIIB) referred to the Radiation Oncology department for curative treatment 
(between May 2006 and September 2012) were selected for the development dataset. Out of these patients, 262 (76\%) had metastatic lymph nodes. The patients received high-dose radiotherapy (RT) combined with chemotherapy, for which an FDG-PET-CT was available for treatment planning. Clinical follow-up was performed according to national guidelines.

\section{Image acquisition}

Before scanning, patients fasted for minimum of 6 hours. Two different protocols were used: until December 2010, the total dose of FDG was calculated as (bodyweight x $4+$ 20) $\mathrm{MBq}$, but as from January 2011 , the administered dose was (2.5 $x$ bodyweight) MBq as defined by the NEDPAS protocol [15]. FDG-PET-CT images were taken 60 minutes post injection. Data acquired until December 2006 were gathered on Siemens Biograph 16 CT-PET scanner, and from that time onwards on a Siemens Truepoint 40 CT-PET (Siemens AG, Munich, Germany). An Ordered Subset Expectation Maximisation 2D 4 iterations 8 subsets (OSEM2D 4i8s) algorithm was used for image reconstruction using post-reconstruction $5 \mathrm{~mm}$ Gaussian filtering, and voxel size of $4.0728 \times 4.0728$, and slice thickness of $3(\mathrm{~mm})$. Model-based methods were applied for scatter correction. All PET scans were corrected for attenuation using the mid-ventilation phase of the 4DCT or a 3DCT thorax in case the 4DCT was not of sufficient image quality due to irregular breathing of the patient. All exams were corrected for random events and decay.

\section{Validation cohort}

\section{Patient population}

Using the same criteria as for the development dataset, the validation dataset included 215 stage I-IIIB NSCLC patients, treated with primary radiotherapy between May 2006 and October 2012 at an independent and external institute. In total 115 (53\%) patients were node-positive, of which 50 patients had an available FDG-PET-CT scan prior to radiotherapy.

\section{Image acquisition}

Prior to FDG injection, patients fasted for at least 6 hours. FDG-PET scans were performed 60 minutes after intravenous injection of approximately $250 \mathrm{MBq}$ FDG (Covidien, Petten, the Netherlands) and $10 \mathrm{mg}$ furosemide. PET scans were performed on Siemens Biograph Duo (Siemens Medical Solutions USA, Inc.) using threedimensional emissions of 4 minutes per bed position as described previously [16]. A low-dose CT scan for localisation and attenuation-correction purposes was acquired. Scanning parameters included $40 \mathrm{~mA} \cdot \mathrm{s}$ ( $50 \mathrm{~mA} \cdot \mathrm{s}$ for patient weight $>100 \mathrm{~kg}$ and $60 \mathrm{~mA} \cdot \mathrm{s}$ if $>120 \mathrm{~kg}), 130 \mathrm{kV}, 5-\mathrm{mm}$ slice collimation, 0.8 -second rotation time, and pitch of 1.5, reconstructed with 3-mm slices for smooth coronal representation. An OSEM2D 4i16s algorithm was used for PET image reconstruction, with a voxel size of $5.3 \times 5.3 \times 3.375$ 
(in $\mathrm{mm}$ ). All PET scans were corrected for attenuation using CT and simulation approaches. Model-based methods were applied for scatter correction. All exams were corrected for random events and decay.

\section{Regions of interest (ROI)}

Images were imported into the research treatment planning system Xio/Focal (development dataset) and Eclipse (validation dataset) using the DICOM protocol. The primary gross tumour volume (tumour) and metastatic hilar/mediastinal lymph nodes (LN), identified as PET positive and/or proven by EBUS/EUS, were manually delineated by experienced radiation oncologists on the fused FDG-PET-CT images, and used as the regions of interest for analysis.

\section{Image analysis}

Software developed in-house was used to extract Radiomics descriptors from the FDGPET scans [17-21]. Imaging descriptors comprised first order statistics ( $n=16)$, shape and size $(n=13)$, intensity volume histograms $(n=45)$, and textural features describing the spatial distribution of voxel intensities $(n=44)$. Textural features were calculated from grey-level co-occurrence (GLCM), grey-level run-length (GLRLM) and grey-level sizezone texture matrices (GLSZM). To determine these matrices, images were first discretised with a bin width of 0.5 (SUV), according to:

$$
I_{D}(x)=\left\lceil\frac{I(x)}{0,5}\right\rceil-\min \left(\left\lceil\frac{I(x)}{0,5}\right\rceil\right)+1
$$

Where I is the original image, I $(x)$ represents the SUV of voxel $x$, and ID is the resulting discretised image [20]. Texture matrices were then constructed by considering 26 connected voxels (i.e. voxels were considered to be neighbours in all 13 directions in three dimensions) at a distance of 1 voxel. Features derived from GLCM and RLGL were calculated by averaging their value over all 13 directions. Forty-four textural features were extracted (22 GLCM, 11 RLGL and 11 GLSZM). In total, 118 imaging features were calculated based on the FDG-PET distribution within ROI, which mathematical formulations can be found elsewhere $[18,19]$. Image analysis was performed in Matlab R2012b (The Mathworks, Natick, MA), based on an adapted version of Computational Environment for Radiotherapy Research (CERR) [22].

\section{Statistical analysis}

\section{Study parameters/endpoints}

The primary endpoint of this study was overall survival (OS), defined as the time from the start of radiotherapy until the last day of follow-up or death due to any cause, and 
was available for all patients under analysis. A patient still alive at the end of the study was regarded as right-censored.

\section{Univariable analysis}

Robust and stable PET-Radiomics features, found to be thoroughly described in the online appendix, were analysed as continuous variables in a univariable Cox regression. In addition, a correlation analysis was conducted on maximum, peak, and mean SUV, and volume, for tumour, all metastatic LNs, and tumour load, which was defined as the union of the tumour and LN volumes.

\section{Multivariable analysis}

A prognostic model was fitted to the data with a 10-fold cross-validated least absolute shrinkage and selection operator (LASSO), selecting amongst the stable and robust PETRadiomics descriptors extracted from the tumour (model 1), LN (model 2) and the union of both structures (model 3) [23]. A diagram illustrating this methodology is shown in Figure 1. Log-likelihood tests for non-nested models, Akaike information criterion (AIC), were performed to compare the fit of the three derived models. AIC measures the relative quality of model fit to a given dataset, providing substantiation for model selection, i.e. for being the preferred model, which is the one with a lower AIC [24]. Log-linearity assumption was verified for the selected features in the final models by fitting a penalised smoothing spline. A Cox proportional hazards assumption was graphically examined for the Schoenfeld residuals.

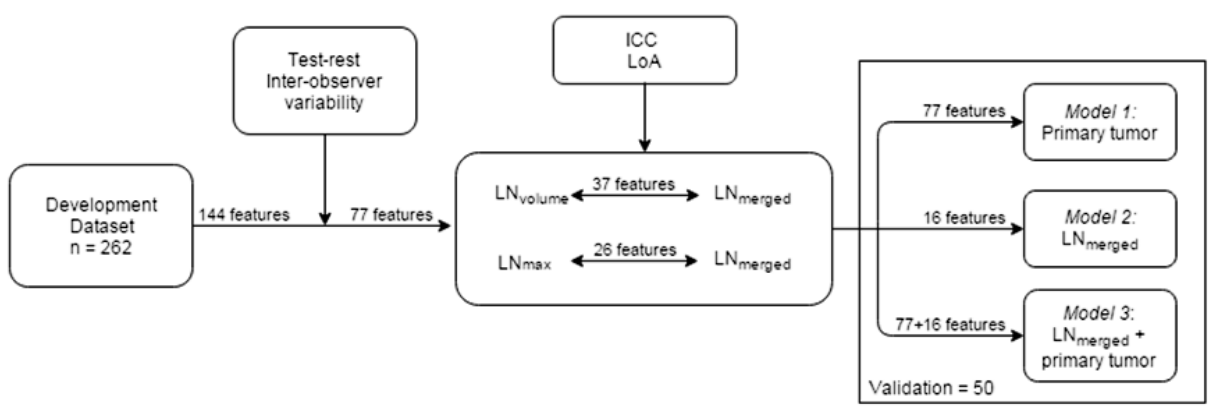

Figure 1 - Diagram of the workflow followed in the multivariable model development phase. After a testretest and inter-observer study, 77 features remained for further analysis, based on a cut-off of 0.85 for the ICC analysis [19]. Further identification of comparable features extracted from a structure merging all metastatic lymph nodes (LNmerged) to the largest (LNvolume) or most active node (LNmax), by means of an intraclass correlation (ICC) over 0.85 and $\pm 10 \%$ limits of agreement (LOA) between measurements, was performed (further details in the online appendix). In summary, 77 features of the primary tumour and 16 from the metastatic lymph nodes were entered in the model development phase. 


\section{Model performance}

Model performance was assessed in the development and validation datasets by means of a concordance-index [25]. Concordance-index, also referred to as Harrell's C-index, evaluates the fraction of patient pairs for which the predicted and actual outcome are concordant, ranging from a random 0.5 to a perfect 1 [26].

A 5\% significance level was used in the analysis. All statistical analysis was conducted in R (version 2.15.2), using the libraries: survival, survcomp, glmnet, cvTols and rms [27]. The development dataset is publicly available at www.cancerdata.org.

\section{RESULTS}

Table 1 gives a complete overview of patients under analysis including treatment details. Node-positive patients with available FDG-PET scans were included in the analysis: 262 for the development dataset and 50 cases for the validation datasets. A univariable analysis was performed for each of the clinical variables in the development dataset (Table 2). Results show that TNM staging was not correlated to OS, while $\mathrm{N}$ stage was a prognostic factor at 0.1 level $(p=0.09)$ in the development dataset, with later stages being associated with a worse prognosis. The number of LN stations was significantly associated with a higher risk in this cohort of patients. None of the remaining analysed metrics, including gender, age or histology showed a significant correlation to OS in our development cohort. 


\section{CHAPTER 7}

Table 1 - Demographics and clinical information of development and validation datasets for analysis of NSCLC.

\begin{tabular}{|c|c|c|c|c|}
\hline \multirow[b]{2}{*}{ Age } & \multicolumn{2}{|c|}{ Development dataset $(n=262)$} & \multicolumn{2}{|c|}{ Validation dataset $(n=50)$} \\
\hline & & & & \\
\hline Mean \pm SD & \multicolumn{2}{|c|}{$66 \pm 10$} & \multicolumn{2}{|c|}{$64 \pm 10$} \\
\hline Range & \multicolumn{2}{|c|}{$33-86$} & \multicolumn{2}{|c|}{$44-83$} \\
\hline \multicolumn{5}{|l|}{ Gender } \\
\hline Male & 172 & $65.6 \%$ & 31 & $62 \%$ \\
\hline Female & 90 & $34.4 \%$ & 19 & $38 \%$ \\
\hline \multicolumn{5}{|l|}{ Stage } \\
\hline II & 10 & $3.8 \%$ & - & - \\
\hline IIla & 107 & $40.8 \%$ & 32 & $64 \%$ \\
\hline $\mathrm{IIlb}$ & 144 & $55 \%$ & 18 & $36 \%$ \\
\hline No information* & 1 & $0.4 \%$ & 2 & $4 \%$ \\
\hline \multicolumn{5}{|l|}{ N stage } \\
\hline 1 & 28 & $10.7 \%$ & 1 & $2 \%$ \\
\hline 2 & 151 & $57.6 \%$ & 36 & $72 \%$ \\
\hline 3 & 80 & $30.5 \%$ & 6 & $12 \%$ \\
\hline No information & 3 & $1.2 \%$ & 7 & $14 \%$ \\
\hline \multicolumn{5}{|l|}{ Number of metastatic LN stations } \\
\hline Mean \pm SD & \multicolumn{2}{|c|}{$3.6 \pm 2.4$} & \multicolumn{2}{|c|}{$2.1 \pm 1.1$} \\
\hline Range & \multicolumn{2}{|c|}{$1-12$} & \multicolumn{2}{|c|}{$1-6$} \\
\hline \multicolumn{5}{|l|}{ Histology } \\
\hline Adenocarcinoma & 60 & $22.9 \%$ & 19 & $38 \%$ \\
\hline Squamous cell carcinoma & 73 & $27.9 \%$ & 18 & $36 \%$ \\
\hline NSCLC-otherwise specified (NOS) & 123 & $46.9 \%$ & 13 & $26 \%$ \\
\hline No information & 6 & $2.3 \%$ & - & - \\
\hline \multicolumn{5}{|l|}{ Radiotherapy Dose (Gy) } \\
\hline Mean \pm SD & \multicolumn{2}{|c|}{$64.4 \pm 7.5$} & \multicolumn{2}{|c|}{$61.75 \pm 6.1$} \\
\hline Range & \multicolumn{2}{|c|}{$45-99.75^{* *}$} & \multicolumn{2}{|c|}{$45-70 * *$} \\
\hline \multicolumn{5}{|l|}{ Chemotherapy } \\
\hline Yes & 227 & $86.6 \%$ & 33 & $66 \%$ \\
\hline No & 25 & $9.6 \%$ & - & - \\
\hline No information & 10 & $3.8 \%$ & 17 & $34 \%$ \\
\hline
\end{tabular}

* If no further information about stage was available in the EMD, TNM was reviewed and stage N0 and M1 patients were excluded from analysis

** Only 6 out of the 262 patients from the development dataset and 2 out of the 50 patients in the validation dataset received a dose under $50 \mathrm{~Gy}$. Based on an individual assessment of the medical records of each of these patients, we could find no evidence to justify removing these from the final analysis. 
Table 2 - Univariable Cox regression of clinical variables in development dataset.

\begin{tabular}{|c|c|c|c|}
\hline Feature & Hazard Ratio & $95 \% \mathrm{Cl}$ & $p$-value \\
\hline Age & 0.99 & $0.97-1.00$ & 0.10 \\
\hline \multicolumn{4}{|l|}{ Gender } \\
\hline Male & Reference & & \\
\hline Female & 0.85 & $0.63-1.15$ & 0.30 \\
\hline \multicolumn{4}{|l|}{ Stage } \\
\hline II & Reference & & \\
\hline IIIa & 1.05 & $0.48-2.28$ & \multirow{2}{*}{0.92} \\
\hline $\mathrm{IIlb}$ & 1.06 & $0.49-2.28$ & \\
\hline \multicolumn{4}{|l|}{ N stage } \\
\hline 1 & Reference & & \\
\hline 2 & 1.44 & $0.86-2.40$ & \multirow{2}{*}{0.09} \\
\hline 3 & 1.75 & $1.02-2.99$ & \\
\hline \multicolumn{4}{|l|}{ Number of metastatic LN stations } \\
\hline 1 & Reference & & \multirow{4}{*}{$<0.01$} \\
\hline 2 & 2.08 & $1.30-3.30$ & \\
\hline 3 & 1.65 & $0.98-2.99$ & \\
\hline$\geq 4$ & 1.95 & $1.28-2.98$ & \\
\hline \multicolumn{4}{|l|}{ Histology } \\
\hline Squamous cell carcinoma & Reference & & \\
\hline Adenocarcinoma & 0.93 & $0.61-1.42$ & \multirow{2}{*}{0.18} \\
\hline NSCLC-otherwise specified (NOS) & 1.26 & $0.89-1.78$ & \\
\hline
\end{tabular}

Results of univariable Cox regression of FDG-PET Radiomics features extracted from both tumour and LNs are shown in the appendix (appendix). Only short run emphasis from the texture RLGL group was significantly correlated to OS when extracted from the primary tumour in the development set. On the other hand, metrics derived from LN showed a good univariable correlation to outcome, with 13 of the 16 analysed features being significantly related to overall survival. Table 3 gives a sub-analysis based on commonly assessed metabolic features (maximum, peak, and mean SUV) and volume of tumour and LNs, for which a high Pearson correlation between metabolic features within each structure could be verified, but no correlation could be found with own volume, nor with metabolic features of the other structure (Figure 2). 
Table 3 - Distribution of common PET descriptors (maximum, peak and mean) and volume of the primary tumour and LNs analysed. Univariable Cox regression of common FDG-PET descriptors extracted from primary tumour (Tumour) and metastatic lymph nodes (Nodes) of the development dataset.

\begin{tabular}{lllllll}
\hline Structure & Features & $\begin{array}{l}\text { Range } \\
\text { (Mean } \pm \text { SD) }\end{array}$ & $\begin{array}{l}\text { Hazard } \\
\text { Ratios }\end{array}$ & p-value & $95 \% \mathrm{Cl}$ & C-index \\
\hline Tumour & Maximum SUV & $1.0-32.5(10.7 \pm 5.7)$ & 1.00 & 0.95 & $0.97-1.03$ & 0.51 \\
& Peak SUV & $0.8-29.5(8.6 \pm 4.9)$ & 1.00 & 0.92 & $0.97-1.03$ & 0.51 \\
& Mean SUV & $0.3-15.6(4.4 \pm 2.3)$ & 0.99 & 0.73 & $0.92-1.06$ & 0.53 \\
& Volume & $0.3-702.4(79.5 \pm 104.6)$ & 1.00 & 0.47 & $1.00-1.00$ & 0.51 \\
\multirow{2}{*}{ Nodes } & Maximum SUV & $1.2-39.8(8.3 \pm 5.4)$ & 1.05 & $<0.01$ & $1.02-1.08$ & 0.58 \\
& Peak SUV & $1.0-32.1(6.4 \pm 4.4)$ & 1.06 & $<0.01$ & $1.03-1.10$ & 0.58 \\
& Mean SUV* & $0.5-14.8(3.5 \pm 1.9)$ & 1.14 & $<0.01$ & $1.06-1.23$ & 0.57 \\
& Volume & $0.7-325.9(35.3 \pm 42.9)$ & 1.01 & $<0.01$ & $1.00-1.01$ & 0.60 \\
& Tumour Load & $3.8-709.6(114.8 \pm 111.3)$ & 1.01 & 0.03 & $1.00-1.00$ & 0.58 \\
\hline
\end{tabular}

* Mean SUV is a generalisation of the mean SUV distribution across all independent metastatic lymph nodes, as extracted from a structure merging all nodes; Total volume refers to the combined volume of the primary tumour and metastatic lymph nodes

Three model approaches were derived which were fitted to the present data. These are described in Table 4. The selected features were log-linear, with the exception of LN volume, which had to be converted into a logarithmic scale. The proportional hazards assumption was satisfied for all features. A graphical representation of these characteristics is available online. Automatic feature selection converged to the single metric of the RLGL group (short run emphasis), being significantly related to outcome in the univariable analysis for model 1 , with a C-index of 0.53 and an external validation of 0.54 . Model 2 used selected features: total LN volume and the surface to volume ratio (shape), histogram uniformity (first order statistics), grey level non-uniformity and short run high grey level emphasis (RLGL of the textural group), reaching a C-index of 0.62 with an external validation of 0.56 . Finally, model 3 selected the same feature as model 1 and four features from the LN, replacing short run high grey level emphasis - RLGL, by entropy - GLCM, and reached a performance of 0.62, and 0.59 in the external cohort. No metrics from the IVH sub-category were selected from any of the analysed structures for the derived models. Based on an AIC test, model 3 (1854.5) was shown to be a better fit than model 2 (1857.4), which itself was already a more precise fit compared to model 1 (1876.4). In summary, the addition of nodal imaging information resulted in a better model fit, compared to a model based exclusively on features derived from the primary tumour. 
Table 4 - Cox regression model for CT-based features extracted from pre-radiotherapy scans of NSCLC patients. Analysis was conducted for primary tumour (Tumour) and metastatic lymph nodes (Nodes) separately, and for both structures in combination. Performance of the model is expressed with an internal and external C-index.

\begin{tabular}{|c|c|c|c|c|c|c|c|}
\hline \multirow[b]{2}{*}{ Model } & \multirow[b]{2}{*}{ Features } & \multicolumn{3}{|c|}{$\begin{array}{l}\text { Tumour and nodes } \\
\text { separately }\end{array}$} & \multicolumn{3}{|c|}{$\begin{array}{l}\text { Tumour and nodes } \\
\text { combined }\end{array}$} \\
\hline & & $\begin{array}{l}\text { Hazard } \\
\text { Ratios }\end{array}$ & p-value & C-index & $\begin{array}{l}\text { Hazard } \\
\text { Ratios }\end{array}$ & p-value & C-index \\
\hline Tumour & RLGL - Short Run Emphasis & 0.13 & 0.04 & $\begin{array}{c}0.53 \\
0.54^{* *}\end{array}$ & 0.06 & 0.01 & \\
\hline \multirow[t]{6}{*}{ Nodes } & Shape-Volume* & 0.93 & 0.47 & & 0.88 & 0.28 & \\
\hline & RLGL - Grey Level Non-uniformity & 1.00 & 0.02 & & 1.00 & 0.02 & $\begin{array}{c}0.62 \\
50 * *\end{array}$ \\
\hline & RLGL - Short Run High Grey Level Emphasis & 51.03 & 0.83 & $0.56^{* *}$ & - & - & . \\
\hline & GLCM - Entropy & - & - & & 1.17 & 0.48 & \\
\hline & Shape - Surface/Volume & 0.90 & 0.41 & & 0.94 & 0.67 & \\
\hline & Stats - Uniformity & 0.10 & 0.06 & & 0.08 & 0.19 & \\
\hline
\end{tabular}

Acronyms: GLCM - Grey Level Co-occurrence matrices; RLGL - Run-length Grey Level matrices; Stats - first order statistics

* A logarithmic transformation was applied to LN volume

** External validation

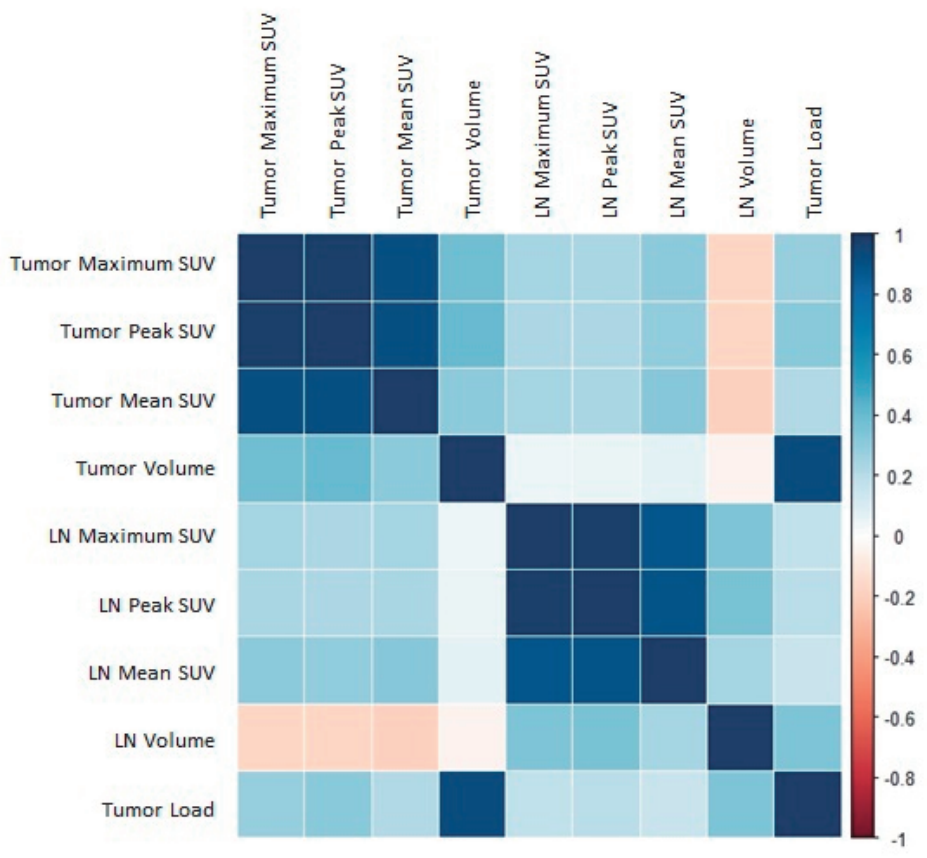

Figure 2 - Pearson correlation plot for metabolic descriptors and volume of primary tumour and metastatic lymph nodes in the development dataset. 


\section{DISCUSSION}

Disease management of NSCLC is a primary concern, for which prognostic assessment is essential to fulfil the potential of individualised and personalised treatment. Nowadays, a wide range of information sources are available of which the non-invasive types, in particular imaging, play a fundamental role in reducing the patients' burden [11-14]. In this study we correlated PET-Radiomics descriptors of metabolic activity based on FDG uptake patterns from both the primary tumour and metastatic lymph nodes with overall survival. Radiomics has been proven to have prognostic potential in predicting clinical outcomes or treatment monitoring in different cancer types [17, 21, 28, 29]. Radiomics can essentially be applied to different medical imaging modalities and disease-related structures such as the primary tumour, metastatic lymph nodes or metastatic lesions. Based on the rationale that disease progression and the ability to metastasise are closely related to the presence of metastatic lymph nodes, we hypothesised that FDG-PETbased Radiomics information of these nodes would provide additional prognostic information in addition to the information that is obtained from the primary tumour [30].

Given the broad range of imaging descriptors which can be analysed with Radiomics, an initial step in this analysis was aimed at investigating the univariable potential of the most commonly analysed PET metrics when extracted from tumour and LNs, namely maximum, peak, and mean SUV, volume and tumour load. None of metabolic metrics extracted from tumour had significant prognostic value, whereas the same ones extracted from LNs were related to OS and yielded an univariable C-index of at least 0.57 (Table 3). No statistical significance could be associated with tumour volume $(p=0.47)$, as opposed to LN volume ( $p<0.01$; $c$-index 0.60). Tumour load, the sums of volumes of tumour and LNs, also had prognostic value, in line with previous studies [31]. Despite the strong correlation between metabolic features within each structure, no correlation with own volume, or metabolic features of the other structure could be found, contrary to previous evidence of correlation between maximum SUV of tumour and total LN volume [32]. One can already infer from this univariable analysis the considerable prognostic value that the metabolic distribution within metastatic lymph nodes has for node-positive NSCLC patients, which is further reinforced by the remaining features analysed.

Of the three independent fits, the model with features from LNs alone or LNs in combination with primary tumour performed best (0.62). The model included at least one feature from categories of shape and size, first order statistics and texture descriptor, excluding features from the IHV group. The intrinsic heterogeneity described by the FDG distribution within a primary tumour has proven its prognostic power, not only for NSCLC, but also for other cancer types [33, 34]. Inclusion of such descriptors in derived prognostic models, gathering heterogeneity insight into not only primary tumour but also metastatic lymph nodes, resulted in a model with a better fit and more accurate description of disease structures. Shape descriptors, including total LN volume, 
included in the multivariable model, were shown to be an independent prognostic parameter. In a previous study, tumour load was revealed to be a prognostic factor, but not LN volume independently, which we proved in this study [31]. Finally, and despite their univariate prognostic value, particularly when assessed from LN, none of the most common SUV descriptors were included in the final models. However, as Radiomics analysis includes a large number of features, selection of the most promising ones is difficult. We attempted to overcome this difficulty using a LASSO approach and external validation. Nonetheless, it cannot be completely excluded that other variables have similar or even greater prognostic value than the current ones, and therefore larger imaging datasets are needed to validate and confirm our findings. Similarly, another limitation of our study was the lack of additional patient data for the validation phase. A lower performance is commonly observed when validating a model against new, independent, and external cohorts, which is most frequently attributed to discrepancies between development and validation data [35]. A larger external dataset which would increase the robustness of the validation procedure would be beneficial. Nevertheless, we observed a benefit from combining imaging features from both primary tumour and metastatic lymph nodes for node-positive NSCLC patients. In terms of prognostics, this should not be disregarded, particularly when compared to the limited capacity of humans to infer an accurate prognosis from same data [36].

A recent study with 139 NSCLC patients treated with at least 60Gy with a concurrent (chemo)radiotherapy regimen showed the importance of measuring the post-treatment SUV in the metastatic lymph nodes, as an increase in both the absolute value and percentage of residual activity compared to the pre-treatment SUV were associated with worse local-regional control [37]. In our study we looked only at the pre-treatment scan and could already discern an association with OS of imaging features derived from the metastatic lymph nodes that could not be assessed based on the primary tumour for node-positive patients. However, we are planning extension of this analysis to include the variation of FDG-PET-Radiomics features between subsequent scans at an early phase of treatment for both the primary tumour and the metastatic lymph nodes, and their impact on survival for NSCLC patients as a complement to the positive findings reported here. Also, a positive correlation between PET information derived from LNs and overall relapse has been reported [38]. Our analysis focused primarily on overall survival, and therefore we could not validate these findings. In the future we will be able to analyse this outcome as we are currently improving our data collection routines, to further evaluate other outcomes, not just in terms of overall survival. Nevertheless, our findings emphasise the importance of analysing FDG-PET signal of metastatic lymph nodes prior to radiotherapy, which can provide further prognostic power beyond the classical TNM classification of the disease, which proved not to be prognostic in our cohort.

Based on the positive and relevant findings we documented, we decided to extend our analysis in a similar manner to other disease sites, particularly head and neck can- 
cer, for which the involvement of the nodes is a well-known prognostic risk factor [39]. Likewise, we are also aiming to analyse other PET tracers, particularly hypoxia markers (e.g. HX4) and compare the results with FDG uptake [40, 41].

\section{CONCLUSION}

In summary, we proved in a large dataset that common SUV descriptors derived from the metastatic lymph nodes are associated with overall survival in NSCLC patients. Additionally, PET information has been shown to have higher prognostic value when extracted from metastatic lymph nodes in comparison to the primary tumour alone, further complementing that information.

\section{ACKNOWLEDGMENTS}

The authors acknowledge financial support of the QuIC-ConCePT project, which is partly funded by EFPIA companies and the Innovative Medicine Initiative Joint Undertaking (IMIJU) under Grant Agreement No. 115151. This research was also funded in part by the Dutch technology Foundation STW (grant $n^{\circ} 10696$ DuCAT), which is the applied science division of the Netherlands Organisation for Scientific Research (NWO), and the Technology Programme of the Ministry of Economic Affairs. Authors also acknowledge the financial support of the CTMM framework (AIRFORCE project, grant 030-103), EU 7th framework programme (EURECA, ARTFORCE - $n^{\circ}$ 257144, REQUITE - $n^{\circ}$ 601826), SME Phase 2 (EU proposal 673780 - RAIL), Kankeronderzoekfonds Limburg from the Health Foundation Limburg and the Dutch Cancer Society (KWF UM 2008-4210, KWF UM 2009-4454). 


\section{REFERENCES}

[1] Dehing-Oberije C, De Ruysscher D, van der Weide $H$, et al. Tumour volume combined with number of positive lymph node stations is a more important prognostic factor than TNM stage for survival of nonsmall-cell lung cancer patients treated with (chemo)radiotherapy. Int I Radiat Oncol Biol Phys 2008;70(4):1039-44.

[2] De Ruysscher D, Wanders S, van Haren E, et al. Selective mediastinal node irradiation based on FDG-PET scan data in patients with non-small-cell lung cancer: a prospective clinical study. Int J Radiat Oncol Biol Phys 2005;62(4):988-94.

[3] Teran MD, Brock MV. Staging lymph node metastases from lung cancer in the mediastinum. J Thorac Dis 2014;6(3):230-6.

[4] Hellwig D, Graeter TP, Ukena D, et al. 18F-FDG PET for mediastinal staging of lung cancer: which SUV threshold makes sense? J Nucl Med 2007;48(11):1761-6.

[5] Lee AY, Choi SJ, Jung KP, et al. Characteristics of Metastatic Mediastinal Lymph Nodes of Non-Small Cell Lung Cancer on Preoperative F-18 FDG PET/CT. Nucl Med Mol Imaging 2014;48(1):41-6.

[6] Mirsadraee S, Oswal D, Alizadeh Y, et al. The 7th lung cancer TNM classification and staging system: Review of the changes and implications. World J Radiol 2012;4(4):128-34.

[7] van Baardwijk A, Bosmans G, Boersma L, et al. PET-CT-based auto-contouring in non-small-cell lung cancer correlates with pathology and reduces interobserver variability in the delineation of the primary tumour and involved nodal volumes. Int J Radiat Oncol Biol Phys 2007;68(3):771-8.

[8] Paesmans M, Garcia C, Wong CO, et al. Primary tumour standardised uptake value is prognostic in nonsmall cell lung cancer: a multivariate pooled analysis of individual data. Eur Respir J 2015; 10.1183/ 13993003.00099-2015.

[9] de Groot PM, Carter BW, Betancourt Cuellar SL, et al. Staging of lung cancer. Clin Chest Med 2015;36(2): 179-96, vii-viii.

[10] Lambin P, Rios-Velazquez E, Leijenaar R, et al. Radiomics: extracting more information from medical images using advanced feature analysis. Eur J Cancer 2012;48(4):441-6.

[11] Lambin P, van Stiphout RG, Starmans MH, et al. Predicting outcomes in radiation oncology--multifactorial decision support systems. Nat Rev Clin Oncol 2013;10(1):27-40.

[12] Lambin P, Roelofs E, Reymen B, et al. 'Rapid Learning health care in oncology' - an approach towards decision support systems enabling customised radiotherapy'. Radiother Oncol 2013;109(1):159-64.

[13] Lambin P, Petit SF, Aerts HJ, et al. The ESTRO Breur Lecture 2009. From population to voxel-based radiotherapy: exploiting intra-tumour and intra-organ heterogeneity for advanced treatment of non-small cell lung cancer. Radiother Oncol 2010;96(2):145-52.

[14] Lambin P, Zindler J, Vanneste B, et al. Modern clinical research: How rapid learning health care and cohort multiple randomised clinical trials complement traditional evidence based medicine. Acta Oncol 2015;54(9):1289-300.

[15] Boellaard R, Oyen WJ, Hoekstra CJ, et al. The Netherlands protocol for standardisation and quantification of FDG whole body PET studies in multi-centre trials. Eur J Nucl Med Mol Imaging 2008;35(12):2320-33.

[16] Schuurbiers OC, Meijer TW, Kaanders JH, et al. Glucose metabolism in NSCLC is histology-specific and diverges the prognostic potential of 18FDG-PET for adenocarcinoma and squamous cell carcinoma. J Thorac Oncol 2014;9(10):1485-93.

[17] Aerts HJ, Velazquez ER, Leijenaar RT, et al. Decoding tumour phenotype by noninvasive imaging using a quantitative radiomics approach. Nat Commun 2014;5:4006.

[18] Carvalho S, Leijenaar RT, Velazquez ER, et al. Prognostic value of metabolic metrics extracted from baseline positron emission tomography images in non-small cell lung cancer. Acta Oncol 2013;52(7): 1398-404.

[19] Leijenaar RT, Carvalho S, Velazquez ER, et al. Stability of FDG-PET Radiomics features: an integrated analysis of test-retest and inter-observer variability. Acta Oncol 2013;52(7):1391-7. 
[20] Leijenaar RT, Nalbantov G, Carvalho S, et al. The effect of SUV discretization in quantitative FDG-PET Radiomics: the need for standardized methodology in tumour texture analysis. Sci Rep 2015;5:11075.

[21] Coroller TP, Grossmann P, Hou Y, et al. CT-based radiomic signature predicts distant metastasis in lung adenocarcinoma. Radiother Oncol 2015;114(3):345-50.

[22] Deasy JO, Blanco Al, Clark VH. CERR: a computational environment for radiotherapy research. Med Phys 2003;30(5):979-85.

[23] Tibshirani R. The lasso method for variable selection in the Cox model. Stat Med 1997;16(4):385-95.

[24] Burnham KP, Anderson DR. Multimodel Inference: Understanding AIC and BIC in Model Selection. Sociological methods and research 2004;33:261-304.

[25] Harrell FE, Jr., Lee KL, Mark DB. Multivariable prognostic models: issues in developing models, evaluating assumptions and adequacy, and measuring and reducing errors. Stat Med 1996;15(4):361-87.

[26] Royston P, Altman DG. External validation of a Cox prognostic model: principles and methods. BMC Med Res Methodol 2013;13:33.

[27] Team RC. R: A language and environment for statistical computing. In. R Foundation for Statistical Computing. 2.15.2 ed; 2015.

[28] Rao SX, Lambregts DM, Schnerr RS, et al. Whole-liver CT texture analysis in colorectal cancer: Does the presence of liver metastases affect the texture of the remaining liver? United European Gastroenterol J 2014;2(6):530-8.

[29] Zhang H, Graham CM, Elci O, et al. Locally advanced squamous cell carcinoma of the head and neck: CT texture and histogram analysis allow independent prediction of overall survival in patients treated with induction chemotherapy. Radiology 2013;269(3):801-9.

[30] Paesmans M, Garcia C, Wong CY, et al. Primary tumour standardised uptake value is prognostic in nonsmall cell lung cancer: a multivariate pooled analysis of individual data. Eur Respir J 2015;46(6):1751-61.

[31] Dehing-Oberije C, Yu S, De Ruysscher D, et al. Development and external validation of prognostic model for 2-year survival of non-small-cell lung cancer patients treated with chemoradiotherapy. Int J Radiat Oncol Biol Phys 2009;74(2):355-62

[32] Wang $\mathrm{Y}, \mathrm{Ma} \mathrm{S}$, Dong $\mathrm{M}$, et al. Evaluation of the factors affecting the maximum standardized uptake value of metastatic lymph nodes in different histological types of non-small cell lung cancer on PET-CT. BMC Pulm Med 2015;15:20.

[33] Cook GJ, Yip C, Siddique M, et al. Are pretreatment 18F-FDG PET tumour textural features in non-small cell lung cancer associated with response and survival after chemoradiotherapy? J Nucl Med 2013;54(1): 19-26.

[34] Hatt M, Majdoub M, Vallieres M, et al. 18F-FDG PET uptake characterization through texture analysis: investigating the complementary nature of heterogeneity and functional tumour volume in a multicancer site patient cohort. J Nucl Med 2015;56(1):38-44.

[35] Toll DB, Janssen KJ, Vergouwe $\mathrm{Y}$, et al. Validation, updating and impact of clinical prediction rules: a review. J Clin Epidemiol 2008;61(11):1085-94.

[36] Oberije C, Nalbantov G, Dekker A, et al. A prospective study comparing the predictions of doctors versus models for treatment outcome of lung cancer patients: a step toward individualized care and shared decision making. Radiother Oncol 2014;112(1):37-43.

[37] Markovina S, Duan F, Snyder BS, et al. Regional Lymph Node Uptake of [(18)F]Fluorodeoxyglucose After Definitive Chemoradiation Therapy Predicts Local-Regional Failure of Locally Advanced Non-Small Cell Lung Cancer: Results of ACRIN 6668/RTOG 0235. (1879-355X (Electronic)).

[38] Li H, Becker N, Raman S, et al. The value of nodal information in predicting lung cancer relapse using 4DPET/4DCT. Med Phys 2015;42(8):4727-33.

[39] Prabhu RS, Hanasoge S, Magliocca KR, et al. Lymph node ratio influence on risk of head and neck cancer locoregional recurrence after initial surgical resection: implications for adjuvant therapy. Head Neck 2015;37(6):777-82.

[40] Zegers CM, van Elmpt W, Reymen B, et al. In vivo quantification of hypoxic and metabolic status of NSCLC tumours using [18F]HX4 and [18F]FDG-PET/CT imaging. Clin Cancer Res 2014;20(24):6389-97. 
FDG-PET RADIOMICS OF METASTATIC LYMPH NODES AND PRIMARY TUMOUR IN NSCLC

[41] Pettersen EO, Ebbesen P, Gieling RG, et al. Targeting tumour hypoxia to prevent cancer metastasis. From biology, biosensing and technology to drug development: the METOXIA consortium. J Enzyme Inhib Med Chem 2015;30(5):689-721. 


\section{APPENDIX 1}

This appendix presents a methodology conducted for feature pre-selection for both primary tumour (tumour) and metastatic lymph nodes (LN), and corresponding results and interpretation.

Robust and stable ${ }^{18}$ F-fluorodeoxyglucose (FDG) Positron Emission Tomography (PET) Radiomics features of the primary tumour were selected from a test-retest and inter-observer analysis, using an intraclass correlation coefficient (ICC) analysis [1]. Details of the methodology and corresponding results are presented elsewhere [2]. Features presenting an intraclass correlation coefficient (ICC) over 0.85 in both test-retest and multiple observer settings were defined as robust. The selection procedure resulted in a total of 77 features.

Metastatic lymph nodes were analysed as a single structure combining all the individual metastatic LN stations. We further assessed how feature values extracted from this merged structure (LNmerged) compared to values from the largest (LNvolume) or most active LN (LNmax), regardless of the corresponding lymph node station. To this end, we randomly selected a subgroup of 88 patients (34\%) of the 262 patients in the derivation dataset with lymphadenopathy and identified LNvolume and LNmax, from which the robust and stable PET Radiomics were extracted. These same features were then derived from the radiotherapy planning structure comprising the total nodal volume (LNmerged). The intraclass correlation coefficient (ICC) was calculated between features extracted from LNvolume and LNmerged (A), as well as between features extracted from LNmax and LNmerged (B). The ICC is based on the analysis of variance and assumes values between 0 and 1 , and were further adjusted as by Spearman-Brown, to reflect the means of the different ratters - ICC $(1, k)[1]$. The ICC can be large only if there is no bias and the paired measurements are in close agreement. Hence, we extended comparisons A and B with a 95\% limit of agreement (LoA), based on the methods of Bland and Altman $[3,4]$.

The largest and most active LN was the same for 55 out of the 88 (62.5\%) patients in the sub-analysis. For LNvolume and LNmerged, 37 out of the 77 features yielded an ICC $(1, \mathrm{k})$ over 0.85 and a LoA within the $\pm 10 \%$ interval, while for LNmax and LNmerged this number decreased to 26 features (Table 1), that were condensed in combination into 12 common features between structures. This analysis followed the aim to capture the independent contributions of each independent node in a single feature value, which varies among patients. Some of the derived features are dependent on the Region-of-interest (ROI), that when comprising more than a single independent lymph node, may influence its value. For this reason, we aimed to identify features that, extracted from a single structure, would be comparable if extracted from the largest or most active LN, improving its interpretability. In addition to these features, we included maximum and peak SUV as well as total LN volume and tumour load in the multivariable modelling, following the univariable evidence that a single high uptake region and LN 
volume are prognostic factors (Table 3 in main text), even though these metrics were not gathered from the largest node, but were, obviously, from the most active one.

In summary, a total of 77 stable and robust features from the primary tumour and 16 for LNmerged, were selected for further analysis following the rationale described.

Table 1 - Number of PET features with an ICC $(1, k)$ over 0.85 and within a $\pm 10 \%$ LoA interval as derived from the largest (LNvolume) or more active node (LNmax) and merged structure (LNmerged), for the different groups of features. Stable and robust features were identified previously, which results may be shown between brackets in the header of each sub-category.

\begin{tabular}{llllllll}
\hline Structure & Statistics & Shape & RLGL & GLCM & GLSZM & IVH & Total \\
& $(13 / 16)$ & $(9 / 13)$ & $(8 / 11)$ & $(10 / 22)$ & $(1 / 11)$ & $(36 / 45)$ & $(77 / 118)$ \\
\hline LN $_{\text {volume }}$ & 5 & 1 & 6 & 4 & 0 & 21 & 37 \\
LN $_{\max }$ & 8 & 1 & 6 & 7 & 0 & 4 & 26 \\
\hline
\end{tabular}

Acronyms: RLGL - run-length grey level; GLCM - grey level co-occurrence; GLSZM - grey level size zone matrices; IVH - intensity-volume histograms.

\section{REFERENCES}

[1] Shrout PE, Fleiss JL. Intraclass correlations: uses in assessing rater reliability. Psychol Bull 1979;86(2):4208.

[2] Leijenaar RT, Carvalho S, Velazquez ER, et al. Stability of FDG-PET Radiomics features: an integrated analysis of test-retest and inter-observer variability. Acta Oncol 2013;52(7):1391-7.

[3] Bland JM, Altman DG. Statistical methods for assessing agreement between two methods of clinical measurement. Lancet 1986;1(8476):307-10.

[4] Dewitte K Fau - Fierens C, Fierens C Fau - Stockl D, Stockl D Fau - Thienpont LM, et al. Application of the Bland-Altman plot for interpretation of method-comparison studies: a critical investigation of its practice. (0009-9147 (Print)). 


\section{APPENDIX 2}

The results presented in this appendix, while not essential to the main message of the manuscript, further complement it. Tables 1 and 3 present the univariable analysis of stable and robust Radiomics features from primary tumour for development and validation datasets. Tables 2 and 4 present the univariable analysis of stable and robust PET Radiomics features that were common surrogates as extracted from the merged and largest node as well as the merged and most active node, and therefore entered as continuous variables in the multivariable model building.

Table 1 - Univariable Cox regression on the robust and stable features of the primary tumour from the development dataset $(n=262)$.

\begin{tabular}{llllll}
\hline $\begin{array}{l}\text { Class of } \\
\text { feature }\end{array}$ & Feature & Hazard Ratio & $p$-value & $\begin{array}{l}\text { 95\% Confidence } \\
\text { Interval }\end{array}$ & C-index \\
\hline $\begin{array}{lllll}\text { Shape and } \\
\text { size }\end{array}$ & Compactness & 1.050 & 0.457 & $0.924-1.193$ & 0.511 \\
$(13 / 16)$ & Maximum Diameter 2Dx & 1.017 & 0.468 & $0.971-1.066$ & 0.508 \\
& Maximum Diameter 2Dy & 1.013 & 0.580 & $0.967-1.062$ & 0.510 \\
& Maximum Diameter 2Dz & 1.033 & 0.232 & $0.980-1.088$ & 0.524 \\
& Maximum Diameter 3D & 1.032 & 0.140 & $0.990-1.076$ & 0.529 \\
& Surface/Volume & 0.988 & 0.850 & $0.873-1.119$ & 0.508 \\
& Surface & 1.001 & 0.302 & $0.999-1.002$ & 0.515 \\
& Volume & 1.000 & 0.470 & $0.999-1.002$ & 0.514 \\
First Order & Number of voxels & 1.000 & 0.397 & $0.999-1.000$ & 0.519 \\
Statistics & Entropy & 1.000 & 0.379 & $0.999-1.000$ & 0.500 \\
& Maximum & 0.922 & 0.305 & $0.790-1.077$ & 0.516 \\
& Mean Deviation & 1.001 & 0.950 & $0.975-1.028$ & 0.491 \\
& Mean & 0.979 & 0.761 & $0.854-1.123$ & 0.518 \\
& Median & 0.988 & 0.732 & $0.924-1.057$ & 0.527 \\
& Range & 0.989 & 0.736 & $0.924-1.057$ & 0.528 \\
& Root Mean Square & 1.001 & 0.914 & $0.975-1.028$ & 0.494 \\
& Standard Deviation & 0.990 & 0.736 & $0.933-1.050$ & 0.524 \\
& Peak Suv & 0.984 & 0.788 & $0.875-1.107$ & 0.517 \\
& Total Energy & 1.002 & 0.916 & $0.972-1.032$ & 0.490 \\
& Uniformity & 1.000 & 0.452 & $0.999-1.000$ & 0.498 \\
& Variance & 2.883 & 0.095 & $0.831-9.998$ & 0.518 \\
\hline
\end{tabular}




\begin{tabular}{|c|c|c|c|c|c|}
\hline $\begin{array}{l}\text { Class of } \\
\text { feature }\end{array}$ & Feature & Hazard Ratio & $\mathrm{p}$-value & $\begin{array}{l}95 \% \text { Confidence } \\
\text { Interval }\end{array}$ & C-index \\
\hline \multirow{22}{*}{$\begin{array}{l}\text { Texture } \\
(19 / 44)\end{array}$} & \multicolumn{5}{|l|}{ Run-length Grey Level (RLGL) (8/11) } \\
\hline & Grey Level Non-uniformity & 1.000 & 0.347 & $0.999-1.001$ & 0.525 \\
\hline & High Grey Level Run Emphasis & 1.000 & 0.662 & $0.999-1.001$ & 0.480 \\
\hline & Long Run Emphasis & 1.110 & 0.076 & $0.989-1.246$ & 0.529 \\
\hline & Long Run High Grey Level Emphasis & 1.000 & 0.713 & $0.999-1.001$ & 0.478 \\
\hline & Run-length Non-uniformity & 1.000 & 0.487 & $0.999-1.000$ & 0.514 \\
\hline & Run Percentage & 0.207 & 0.055 & $0.042-1.033$ & 0.531 \\
\hline & Short Run Emphasis & 0.131 & 0.043 & $0.018-0.941$ & 0.533 \\
\hline & Short Run High Grey Level Emphasis & 0.995 & 0.790 & $0.961-1.031$ & 0.520 \\
\hline & \multicolumn{5}{|l|}{ Grey Level Co-occurrence (GLCM) (10/22) } \\
\hline & Autocorrelation & 1.000 & 0.682 & $0.999-1.001$ & 0.479 \\
\hline & Cluster Prominence & 1.000 & 0.463 & $0.999-1.000$ & 0.489 \\
\hline & Cluster Tendency & 1.000 & 0.655 & $0.999-1.001$ & 0.488 \\
\hline & Contrast & 1.001 & 0.894 & $0.991-1.010$ & 0.477 \\
\hline & Dissimilarity & 0.964 & 0.512 & $0.864-1.076$ & 0.526 \\
\hline & Entropy & 0.979 & 0.697 & $0.878-1.091$ & 0.512 \\
\hline & Sum Average & 0.998 & 0.768 & $0.982-1.013$ & 0.523 \\
\hline & Sum Entropy & 0.973 & 0.753 & $0.821-1.153$ & 0.511 \\
\hline & Sum of Squares & 1.000 & 0.691 & $0.999-1.001$ & 0.478 \\
\hline & Sum of Variance & 1.000 & 0.643 & $0.999-1.000$ & 0.478 \\
\hline & \multicolumn{5}{|l|}{ Grey Level Size Zone (GLSZM) (1/11) } \\
\hline & High Intensity Large Area Emphasis & 1.000 & 0.958 & $0.999-1.000$ & 0.502 \\
\hline \multirow{16}{*}{$\begin{array}{l}\text { Intensity- } \\
\text { Volume } \\
\text { Histograms } \\
\text { (IVH) } \\
(36 / 45)\end{array}$} & \multicolumn{5}{|c|}{ Absolute Intensity of Relative Volume (AIRV) (9/9) } \\
\hline & AIRV $10 \%$ & 0.996 & 0.824 & $0.958-1.034$ & 0.519 \\
\hline & AIRV $20 \%$ & 0.992 & 0.724 & $0.949-1.037$ & 0.525 \\
\hline & AIRV $30 \%$ & 0.990 & 0.681 & $0.941-1.040$ & 0.528 \\
\hline & AIRV $40 \%$ & 0.987 & 0.669 & $0.932-1.046$ & 0.530 \\
\hline & AIRV $50 \%$ & 0.989 & 0.736 & $0.924-1.057$ & 0.528 \\
\hline & AIRV 60\% & 0.987 & 0.741 & $0.911-1.068$ & 0.529 \\
\hline & AIRV $70 \%$ & 0.982 & 0.705 & $0.891-1.081$ & 0.527 \\
\hline & AIRV 80\% & 0.982 & 0.763 & $0.870-1.107$ & 0.522 \\
\hline & AIRV 90\% & 0.981 & 0.809 & $0.836-1.150$ & 0.519 \\
\hline & \multicolumn{5}{|c|}{ Absolute Volume of Relative Intensity (AVRI) (9/9) } \\
\hline & AVRI $10 \%$ & 1.000 & 0.577 & $0.999-1.002$ & 0.513 \\
\hline & AVRI 20\% & 1.000 & 0.713 & $0.999-1.002$ & 0.512 \\
\hline & AVRI 30\% & 1.000 & 0.873 & $0.998-1.002$ & 0.509 \\
\hline & AVRI $40 \%$ & 1.000 & 0.994 & $0.998-1.002$ & 0.497 \\
\hline & AVRI 50\% & 1.000 & 0.967 & $0.996-1.004$ & 0.497 \\
\hline
\end{tabular}




\begin{tabular}{|c|c|c|c|c|c|}
\hline $\begin{array}{l}\text { Class of } \\
\text { feature }\end{array}$ & Feature & Hazard Ratio & $p$-value & $\begin{array}{l}95 \% \text { Confidence } \\
\text { Interval }\end{array}$ & C-index \\
\hline & AVRI $60 \%$ & 1.001 & 0.854 & $0.994-1.007$ & 0.494 \\
\hline & AVRI 70\% & 1.001 & 0.872 & $0.989-1.013$ & 0.485 \\
\hline & AVRI $80 \%$ & 0.999 & 0.956 & $0.960-1.039$ & 0.522 \\
\hline & AVRI 90\% & 0.958 & 0.701 & $0.770-1.192$ & 0.512 \\
\hline \multicolumn{6}{|c|}{ Mean Intensity of Relative Volume (MIRV) (9/9) } \\
\hline & MIRV $10 \%$ & 0.996 & 0.818 & $0.963-1.031$ & 0.519 \\
\hline & MIRV $20 \%$ & 0.995 & 0.792 & $0.958-1.033$ & 0.520 \\
\hline & MIRV $30 \%$ & 0.994 & 0.761 & $0.954-1.035$ & 0.521 \\
\hline & MIRV $40 \%$ & 0.993 & 0.743 & $0.951-1.037$ & 0.523 \\
\hline & MIRV 50\% & 0.992 & 0.743 & $0.947-1.039$ & 0.524 \\
\hline & MIRV $60 \%$ & 0.992 & 0.738 & $0.944-1.042$ & 0.525 \\
\hline & MIRV $70 \%$ & 0.991 & 0.737 & $0.940-1.045$ & 0.525 \\
\hline & MIRV 80\% & 0.990 & 0.732 & $0.935-1.048$ & 0.526 \\
\hline & MIRV 90\% & 0.989 & 0.735 & $0.930-1.052$ & 0.526 \\
\hline \multicolumn{6}{|c|}{ Total Lesion Glycolysis of Relative Intensity (TLGRI) (9/9) } \\
\hline & TLGRI 10\% & 1.000 & 0.600 & $0.999-1.000$ & 0.505 \\
\hline & TLGRI 20\% & 1.000 & 0.649 & $0.999-1.000$ & 0.504 \\
\hline & TLGRI 30\% & 1.000 & 0.713 & $0.999-1.000$ & 0.502 \\
\hline & TLGRI $40 \%$ & 1.000 & 0.744 & $0.999-1.000$ & 0.498 \\
\hline & TLGRI 50\% & 1.000 & 0.623 & $0.999-1.000$ & 0.494 \\
\hline & TLGRI 60\% & 1.000 & 0.466 & $1.000-1.001$ & 0.490 \\
\hline & TLGRI 70\% & 1.000 & 0.406 & $1.000-1.001$ & 0.484 \\
\hline & TLGRI 80\% & 1.001 & 0.396 & $0.999-1.003$ & 0.479 \\
\hline & TLGRI 90\% & 1.004 & 0.556 & $0.992-1.015$ & 0.485 \\
\hline
\end{tabular}


Table 2 - Univariable Cox regression on the robust and stable features of the metastatic lymph nodes from the development dataset $(n=262)$. Analysis was conducted for features with an $\operatorname{ICC}(1, k)$ over 0.85 and within a $\pm 10 \%$ LoA interval as derived from the largest ( $\left(\mathrm{N}_{\text {volume }}\right)$ and more active node $\left(\mathrm{LN}_{\max }\right)$ and merged structure $\left(\mathrm{LN}_{\text {merged }}\right)$, for the different groups of features, with the exception of the ones indicated by an asterisk $\left({ }^{*}\right)$. Volume corresponds to the total volume of all metastatic lymph nodes $\left({ }^{+}\right)$.

\begin{tabular}{|c|c|c|c|c|c|}
\hline $\begin{array}{l}\text { Class of } \\
\text { feature }\end{array}$ & Feature & Hazard Ratio & $p$-value & $\begin{array}{l}\text { 95\% Confidence } \\
\text { Interval }\end{array}$ & C-index \\
\hline \multirow{2}{*}{$\begin{array}{l}\text { Shape and } \\
\text { size }(1 / 13)\end{array}$} & Volume $^{+}$ & 1.006 & $<0.01$ & $1.004-1.009$ & 0.599 \\
\hline & Surface/Volume & 0.717 & $<0.01$ & $0.611-0.841$ & 0.585 \\
\hline \multirow{5}{*}{$\begin{array}{l}\text { First Order } \\
\text { Statistics } \\
(2 / 16)\end{array}$} & Entropy & 1.358 & $<0.01$ & $1.162-1.586$ & 0.585 \\
\hline & Maximum* & 1.051 & $<0.01$ & $1.024-1.078$ & 0.580 \\
\hline & Mean* & 1.143 & $<0.01$ & $1.060-1.233$ & 0.572 \\
\hline & Peak SUV* & 1.064 & $<0.01$ & $1.031-1.078$ & 0.580 \\
\hline & Uniformity & 0.057 & $<0.01$ & $0.013-0.246$ & 0.587 \\
\hline \multirow{8}{*}{$\begin{array}{l}\text { Texture } \\
(6 / 44)\end{array}$} & \multicolumn{5}{|l|}{ Run-length Grey Level (RLGL) } \\
\hline & Grey Level Non-uniformity & 1.003 & $<0.01$ & $1.001-1.005$ & 0.569 \\
\hline & Long Run Emphasis & 0.942 & 0.601 & $0.752-1.179$ & 0.539 \\
\hline & Short Run Emphasis & 9.561 & 0.061 & $0.904-101.1$ & 0.546 \\
\hline & Short Run High Grey Level Emphasis & 1.080 & $<0.01$ & $1.039-1.124$ & 0.582 \\
\hline & \multicolumn{5}{|l|}{ Grey Level Co-occurrence (GLCM) } \\
\hline & Entropy & 1.290 & $<0.01$ & $1.143-1.455$ & 0.593 \\
\hline & Sum of Average & 1.034 & $<0.01$ & $1.017-1.052$ & 0.580 \\
\hline \multirow{5}{*}{$\begin{array}{l}\text { IVH } \\
(3 / 45)\end{array}$} & \multicolumn{5}{|c|}{ Absolute Volume of Relative Intensity (AVRI) } \\
\hline & AVRI 90\% & 1.428 & 0.070 & $0.971-2.100$ & 0.545 \\
\hline & \multicolumn{5}{|l|}{ Mean Intensity of Relative Volume (MIRV) } \\
\hline & MIRV $10 \%$ & 1.067 & $<0.01$ & $1.030-1.106$ & 0.575 \\
\hline & MIRV $20 \%$ & 1.076 & $<0.01$ & $1.034-1.121$ & 0.575 \\
\hline
\end{tabular}


Table 3 - Univariable Cox regression on the robust and stable features of the primary tumour from the validation dataset $(\mathrm{n}=50)$.

\begin{tabular}{|c|c|c|c|c|c|}
\hline $\begin{array}{l}\text { Class of } \\
\text { feature }\end{array}$ & Feature & Hazard Ratio & $\mathrm{p}$-value & $\begin{array}{l}95 \% \text { Confidence } \\
\text { Interval }\end{array}$ & C-index \\
\hline \multirow{9}{*}{$\begin{array}{l}\text { Shape and } \\
\text { size } \\
(13 / 16)\end{array}$} & Compactness & 1.199 & 0.293 & $0.850-1.680$ & 0.553 \\
\hline & Maximum Diameter 2Dx & 1.037 & 0.576 & $0.910-1.180$ & 0.533 \\
\hline & Maximum Diameter 2Dy & 1.107 & 0.088 & $0.980-1.240$ & 0.576 \\
\hline & Maximum Diameter 2Dz & 1.097 & 0.216 & $0.950-1.270$ & 0.565 \\
\hline & Maximum Diameter 3D & 1.079 & 0.202 & $0.960-1.210$ & 0.561 \\
\hline & Surface/Volume & 0.939 & 0.810 & $0.560-1.570$ & 0.545 \\
\hline & Surface & 1.003 & 0.098 & $1.000-1.010$ & 0.549 \\
\hline & Volume & 1.003 & 0.092 & $1.000-1.010$ & 0.551 \\
\hline & Number of voxels & 1.000 & 0.175 & $0.999-1.000$ & 0.549 \\
\hline \multirow{13}{*}{$\begin{array}{l}\text { First Order } \\
\text { Statistics } \\
(9 / 13)\end{array}$} & Energy & 1.000 & 0.242 & $0.999-1.000$ & 0.555 \\
\hline & Entropy & 1.082 & 0.674 & $0.750-1.560$ & 0.561 \\
\hline & Maximum & 1.023 & 0.217 & $0.990-1.060$ & 0.598 \\
\hline & Mean Deviation & 1.044 & 0.635 & $0.870-1.250$ & 0.583 \\
\hline & Mean & 1.000 & 0.992 & $0.900-1.100$ & 0.500 \\
\hline & Median & 0.978 & 0.696 & $0.880-1.090$ & 0.535 \\
\hline & Range & 1.025 & 0.279 & $0.980-1.070$ & 0.594 \\
\hline & Root Mean Square & 1.023 & 0.213 & $0.990-1.060$ & 0.605 \\
\hline & Standard Deviation & 1.007 & 0.881 & $0.920-1.100$ & 0.519 \\
\hline & Peak SUV & 1.053 & 0.514 & $0.900-1.230$ & 0.590 \\
\hline & Total Energy & 1.000 & 0.280 & $0.999-1.000$ & 0.552 \\
\hline & Uniformity & 0.389 & 0.680 & $0.000-34.55$ & 0.544 \\
\hline & Variance & 1.004 & 0.648 & $0.990-1.020$ & 0.590 \\
\hline \multirow{15}{*}{$\begin{array}{l}\text { Texture } \\
(19 / 44)\end{array}$} & \multicolumn{5}{|l|}{ Run-length Grey Level (RLGL) (8/11) } \\
\hline & Grey Level Non-uniformity & 1.002 & 0.283 & $1.000-1.010$ & 0.533 \\
\hline & High Grey Level Run Emphasis & 1.000 & 0.901 & $0.999-1.000$ & 0.550 \\
\hline & Long Run Emphasis & 0.950 & 0.771 & $0.670-1.340$ & 0.460 \\
\hline & Long Run High Grey Level Emphasis & 1.000 & 0.967 & $0.999-1.000$ & 0.547 \\
\hline & Run-length Non-uniformity & 1.000 & 0.220 & $0.999-1.000$ & 0.552 \\
\hline & Run Percentage & 0.960 & 0.980 & $0.040-22.47$ & 0.543 \\
\hline & Short Run Emphasis & 1.241 & 0.915 & $0.020-64.63$ & 0.460 \\
\hline & Short Run High Grey Level Emphasis & 1.001 & 0.973 & $0.950-1.060$ & 0.526 \\
\hline & \multicolumn{5}{|l|}{ Grey Level Co-occurrence (GLCM) (10/22) } \\
\hline & Autocorrelation & 1.000 & 0.988 & $0.999-1.000$ & 0.539 \\
\hline & Cluster Prominence & 1.000 & 0.428 & $0.999-1.000$ & 0.616 \\
\hline & Cluster Tendency & 1.000 & 0.530 & $0.999-1.000$ & 0.598 \\
\hline & Contrast & 1.001 & 0.739 & $0.999-1.000$ & 0.537 \\
\hline & Dissimilarity & 1.013 & 0.819 & $0.910-1.130$ & 0.517 \\
\hline
\end{tabular}




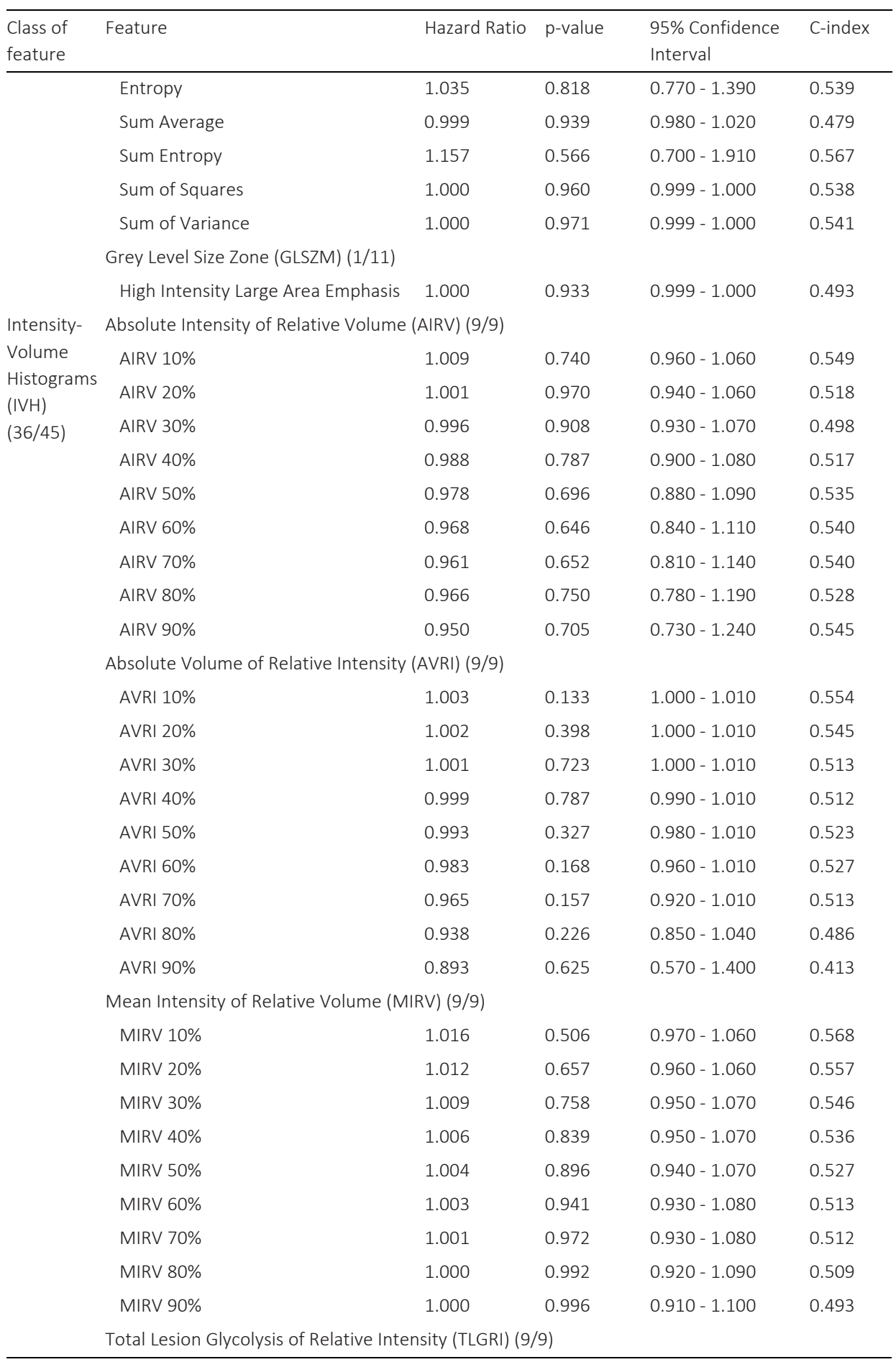




\begin{tabular}{clllll}
\hline $\begin{array}{l}\text { Class of } \\
\text { feature }\end{array}$ & Feature & Hazard Ratio & p-value & $\begin{array}{l}\text { 95\% Confidence } \\
\text { Interval }\end{array}$ & C-index \\
\hline TLGRI 10\% & 1.000 & 0.234 & $0.999-1.000$ & 0.550 \\
TLGRI 20\% & 1.000 & 0.362 & $0.999-1.000$ & 0.534 \\
TLGRI 30\% & 1.000 & 0.565 & $0.999-1.000$ & 0.527 \\
TLGRI 40\% & 1.000 & 0.967 & $0.999-1.000$ & 0.525 \\
TLGRI 50\% & 1.000 & 0.524 & $0.999-1.000$ & 0.490 \\
TLGRI 60\% & 0.999 & 0.268 & $0.999-1.000$ & 0.498 \\
TLGRI 70\% & 0.998 & 0.239 & $0.999-1.000$ & 0.485 \\
TLGRI 80\% & 0.997 & 0.366 & $0.999-1.000$ & 0.460 \\
TLGRI 90\% & 1.002 & 0.862 & $0.980-1.030$ & 0.587 \\
\hline
\end{tabular}

Table 4 - Univariable Cox regression on the robust and stable features of the metastatic lymph nodes from the validation dataset $(n=50)$. Analysis was conducted for features with an $\operatorname{ICC}(1, k)$ over 0.85 and within a $\pm 10 \%$ LoA interval as derived from the largest ( $\left.\mathrm{LN}_{\text {volume}}\right)$ and more active node $\left(\mathrm{LN}_{\max }\right)$ and merged structure $\left(L N_{\text {merged }}\right)$, for the different groups of features, with exception of the ones indicated by an asterisk $\left({ }^{*}\right)$. Volume corresponds to the total volume of all metastatic lymph nodes $\left({ }^{\dagger}\right)$.

\begin{tabular}{|c|c|c|c|c|c|}
\hline $\begin{array}{l}\text { Class of } \\
\text { feature }\end{array}$ & Feature & Hazard Ratio & p-value & $\begin{array}{l}95 \% \text { Confidence } \\
\text { Interval }\end{array}$ & C-index \\
\hline \multirow{2}{*}{$\begin{array}{l}\text { Shape and } \\
\text { size }(1 / 13)\end{array}$} & Volume $^{+}$ & 1.010 & 0.315 & $0.991-1.029$ & 0.593 \\
\hline & Surface/Volume & 0.849 & 0.328 & $0.610-1.180$ & 0.550 \\
\hline \multirow{5}{*}{$\begin{array}{l}\text { First Order } \\
\text { Statistics } \\
(2 / 16)\end{array}$} & Entropy & 1.364 & 0.061 & $.986-1.887$ & 0.593 \\
\hline & Maximum* & 1.064 & 0.023 & $1.009-1.122$ & 0.595 \\
\hline & Mean* & 1.135 & 0.153 & $0.954-1.349$ & 0.572 \\
\hline & Peak SUV* & 1.077 & 0.034 & $1.005-1.153$ & 0.606 \\
\hline & Uniformity & 0.090 & 0.081 & $0.006-1.348$ & 0.579 \\
\hline \multirow{8}{*}{$\begin{array}{l}\text { Texture } \\
(6 / 44)\end{array}$} & \multicolumn{5}{|l|}{ Run-length Grey Level (RLGL) } \\
\hline & Grey Level Non-uniformity & 1.000 & 0.949 & $0.989-1.011$ & 0.568 \\
\hline & Long Run Emphasis & 0.844 & 0.761 & $0.282-2.527$ & 0.537 \\
\hline & Short Run Emphasis & 5.018 & 0.656 & $0.004-6053$ & 0.541 \\
\hline & Short Run High Grey Level Emphasis & 1.076 & 0.107 & $0.984-1.176$ & 0.572 \\
\hline & \multicolumn{5}{|l|}{ Grey Level Co-occurrence (GLCM) } \\
\hline & Entropy & 1.248 & 0.071 & $0.981-1.588$ & 0.598 \\
\hline & Sum of Average & 1.031 & 0.109 & $0.993-1.070$ & 0.570 \\
\hline IVH & \multicolumn{5}{|c|}{ Absolute Volume of Relative Intensity (AVRI) } \\
\hline \multirow[t]{4}{*}{$(3 / 45)$} & AVRI 90\% & 0.937 & 0.896 & $0.355-2.473$ & 0.425 \\
\hline & \multicolumn{5}{|l|}{ Mean Intensity of Relative Volume (MIRV) } \\
\hline & MIRV $10 \%$ & 1.078 & 0.044 & $1.002-1.16$ & 0.582 \\
\hline & MIRV $20 \%$ & 1.085 & 0.060 & $0.997-1.181$ & 0.580 \\
\hline
\end{tabular}



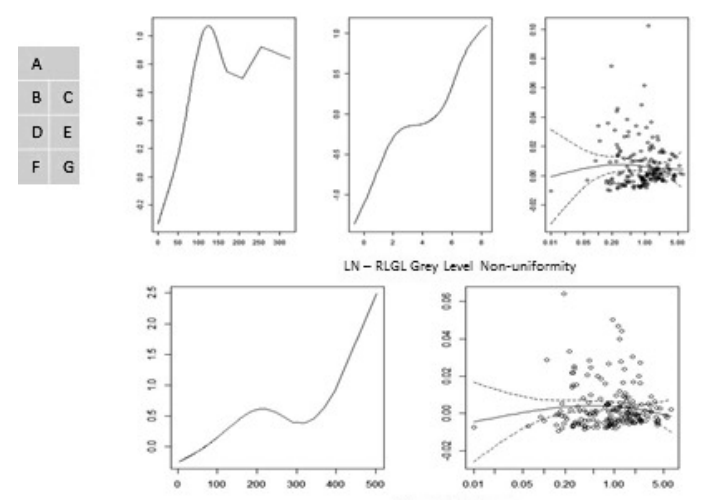

$$
\text { LN - GLCM Entropy }
$$

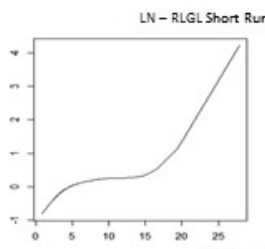

LN - RLGL Short Run High Grey Level Emphasis
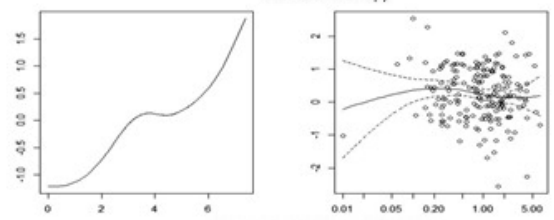

N - First Order Statistics Uniformity
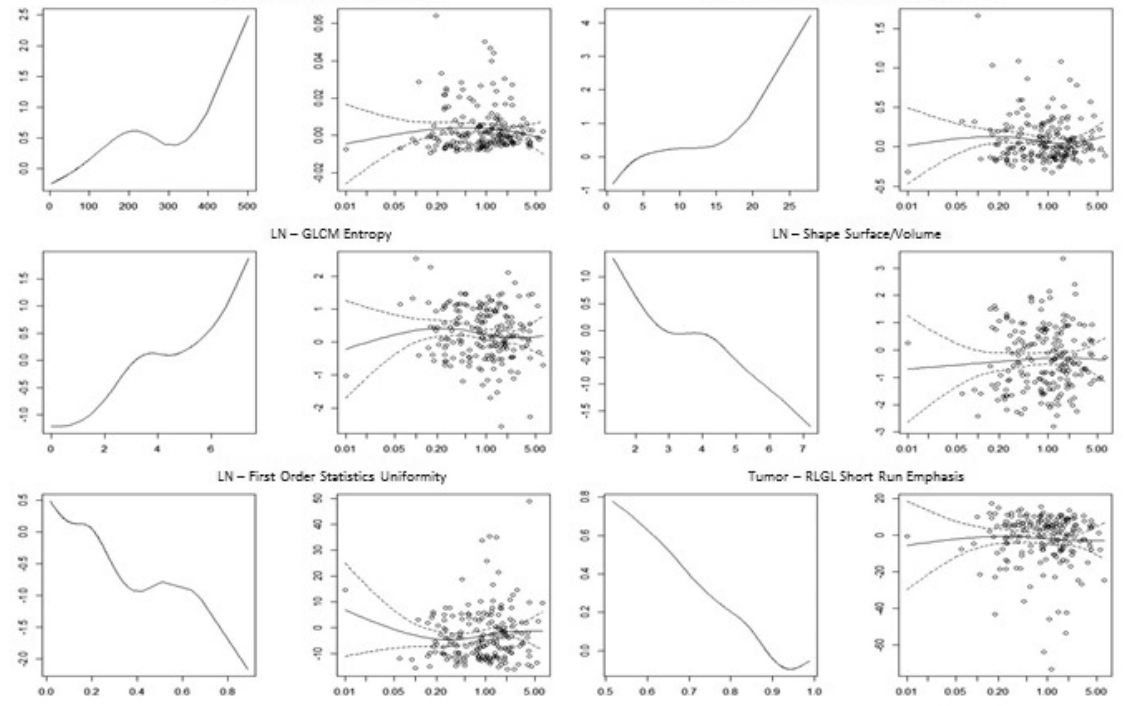

Surface/Nolume
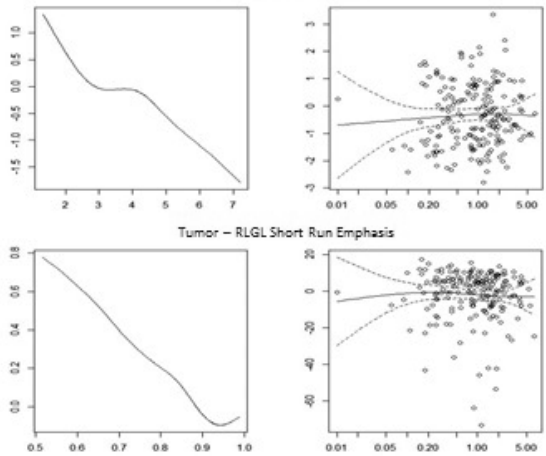

Run Emphasis

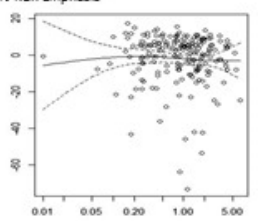

Figure 1 - Log-linear and proportional hazards assumptions verification. Graphically, log-linearity was verified by fitting a penalised smoothing spline on the univariable effect of each variable included in models (left graph), while proportional hazards were analysed by plotting Schoenfeld residuals versus log (time) (right graph). These included variables for $L N$, the (A) volume, (B) RLGL grey level non-uniformity, (C) RLGL short run high grey level emphasis, (D) GLCM entropy, (E) surface to volume ratio, and (F) uniformity, and (G) RLGL short run emphasis of tumour. All variables were log (linear), except LN volume (A left), for which a logarithmic transformation was performed (A middle). All variables satisfied the proportional hazards assumption. Acronyms: LN - metastatic lymph nodes; RLGL - Run-length Grey Level matrices; GLCM - Grey Level Cooccurrence matrices 



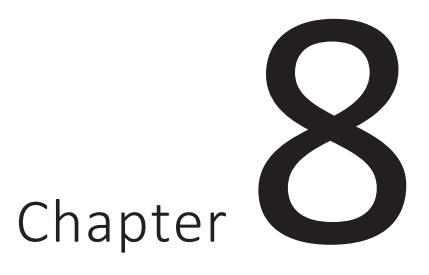

Prognostic value of blood-biomarkers related to hypoxia, inflammation, immune response and tumour load in non-small cell lung cancer

\section{A survival model with external validation}

Accepted for publication at Radiotherapy and Oncology

Prognostic value of blood-biomarkers related to hypoxia, inflammation, immune response and tumour load in non-small cell lung cancer - a survival model with external validation Sara Carvalho, Esther G.C. Troost, Judith Bons, Paul Menheere, Philippe Lambin*, Cary Oberije*

* These authors contributed equally to this work 


\section{ABSTRACT}

Background

Improve the prognostic prediction of clinical variables for non-small cell lung cancer (NSCLC), by selecting from blood-biomarkers, non-invasively describing hypoxia, inflammation and tumour load.

\section{Methods}

Model development and validation included 182 and 181 inoperable stage I-IIIB NSCLC patients treated radically with radiotherapy (55.2\%) or chemo-radiotherapy (44.8\%). Least absolute shrinkage and selection operator (LASSO), selected from bloodbiomarkers related to hypoxia [osteopontin (OPN) and carbonic anhydrase IX (CA-IX)], inflammation [interleukin-6 (IL-6), IL-8, and C-reactive protein (CRP)], and tumour load [carcinoembryonic antigen (CEA), and cytokeratin fragment 21-1 (Cyfra 21-1)]. Sequent model extension selected from alpha-2-macroglobulin (a2M), serum interleukin-2 receptor (sIL2r), toll-like receptor 4 (TLR4), and vascular endothelial growth factor (VEGF). Discrimination was reported by concordance-index.

\section{Results}

OPN and Cyfra 21-1 (hazard ratios of 3.3 and 1.7) significantly improved a clinical model comprising gender, World Health Organization performance-status, forced expiratory volume in $1 \mathrm{~s}$, number of positive lymph node stations, and gross tumour volume, from a concordance-index of 0.66 to 0.70 (validation $=0.62$ and 0.66 ). Extension of the validated model yielded a concordance-index of 0.67 , including a2M, sIL2r and VEGF (hazard ratios of $4.6,3.1$, and 1.4 ).

\section{Conclusion}

Improvement of a clinical model including hypoxia and tumour load blood-biomarkers was validated. New immunological markers were associated with overall survival. Data and models can be found at www.cancerdata.org (http://dx.doi.org/10.17195/candat. 2016.04.1) and www.predictcancer.org. 


\section{INTRODUCTION}

In the last three decades, lung cancer has been the leading cause of cancer deaths [1]. To increase the survival of lung cancer patients, we have witnessed an improvement of radiotherapy techniques and more effective (chemo)radiotherapy schemes (i.e., introduction of concurrent treatment) [2-4]. Attempts have been made to develop more accurate risk stratification for non-small cell lung cancer (NSCLC) patients, which would lead to more tailored, individualised and personalised care, avoiding over or undertreatment, by means of a radiation oncology based on multifactorial Decision Support Systems $[5,6]$. Therefore, the investigation of new prognostic parameters derived from, but not limited to, anatomic, molecular and functional imaging, genomics, and proteomics is warranted [7-9].

The analysis of biomarkers, including proteins, is a fast-developing, promising and challenging area of research, permitting the prediction or description of the evolution of normal biological processes, pathogenic processes, or pharmacological responses to a therapeutic intervention [10]. Oncoproteins are produced by tumour cells or in response to their presence, and may be released into the bloodstream of cancer patients. As tissue sampling is often not possible in lung cancer patients, blood sample collection by venipuncture is an attractive alternative, which is safe and easy to implement [10]. Blood-biomarkers reflect dissimilarities of the tumour microenvironment, are linked to disease prognosis and response to treatment. Blood-biomarkers, that can be measured in daily clinical practice and have been shown to be associated with treatment outcome were first identified in studies comprising large datasets. Based on this criterion, those which biological functions are related to processes of hypoxia [osteopontin (OPN) and carbonic anhydrase IX (CA-IX)]; inflammation [interleukin 6 (IL-6), IL-8, and C-reactive protein (CRP)], and tumour load [carcinoembryonic antigen (CEA) and cytokeratin fragment (CYFRA 21-1)], were analysed and externally validated [11-25]. As an exploratory step we investigated additional blood-biomarkers, including those related to immunological response, which could therefore be incorporated into immunotherapy assessment studies: alpha-2-macroglobulin ( $(2 \mathrm{M})$ ), serum IL-2 receptor (sIL2R), toll-like receptor 4 (TLR4), and vascular endothelial growth factor (VEGF) [14, 26-35].

\section{PATIENTS AND METHODS}

\section{Development dataset}

The development cohort included 195 stage I-IIIb NSCLC patients treated with (chemo)radiotherapy between October 2003 and October 2008. Clinical data and blood samples were prospectively collected to ensure standardisation. Exclusion criteria included surgery or palliative treatment, and insufficient material to perform blood 
measurements (OPN, CA-IX, IL-6, IL-8, CRP, CEA, and CYFRA 21-1). All patients participated in the Biobank project (Clinical trials.gov identifiers: NCT00181519, NCT00573040, and NCT00572325) launched in 2003, and provided written informed consent. One hundred and eighty-two patients were treated according to dissimilar radiotherapy (RT) regimens, with a minimum dose of 50Gy:

1. Forty-nine patients (26.8\%) received the standard external beam radiation therapy (EBRT) protocol used until August 2005, of either 70 Gy (Stage I-II) or 60 Gy after induction chemotherapy (Stage III) in once-daily fractions of $2 \mathrm{~Gy}$.

2. One hundred and one patients (55.2\%) were treated with EBRT only according to the protocol as of August 2005, with an individualised dose delivered in fractions of 1.8 Gy twice daily, until normal tissue dose constraints were met (e.g., mean lung dose, or maximum dose to the spinal cord) [36].

3. Thirty-three patients (18\%) received concurrent (chemo)radiotherapy with a total dose of $45 \mathrm{~Gy}$, delivered in fractions of $1.5 \mathrm{~Gy}$ twice daily, followed by an individualised dose of 8 to 24 Gy delivered in fractions of 2.0 Gy once daily, again limited by the dose to surrounding organs at risk [37].

\section{Validation dataset}

The validation cohort consisted of 200 NSCLC patients with same characteristics as the development cohort, treated between March 2007 and September 2013. Measurements included the above mentioned blood-biomarkers plus VEGF, $\alpha 2 \mathrm{M}, \mathrm{TLR} 4$ and SIL2R (Clinicaltrials.gov identifier: NCT01936571). One hundred and eighty-one patients received a minimum dose of $50 \mathrm{~Gy}$ and were treated as follows:

1. Sixty-eight patients (37.6\%) received radiotherapy alone according to the protocol as of August 2005, with an individualised total dose delivered in fractions of $1.8 \mathrm{~Gy}$ twice daily, limited by the mean lung dose or the spinal cord dose [36].

2. One hundred and one patients (55.8\%) received concurrent chemo-radiotherapy scheme for a prescribed dose of $45 \mathrm{~Gy}$, followed by an individualised dose ranging from 8 to $24 \mathrm{~Gy}$, delivered in fractions of 2.0 Gy once daily, again limited by the dose to surrounding organs at risk [37].

3. Twelve patients (6.6\%) followed the Phase II Positron Emission Tomography (PET) boost trial (clinicaltrals.gov identifier NCT01024829), in which a dose escalation protocol was based on the Fluorine-18-Fluorodeoxyglucose distribution of the PET scans [38]. 


\section{Radiation treatment}

Patients were irradiated in accordance with local protocols and stage of the disease. No elective nodal irradiation was performed and irradiation was delivered 5 days a week [39]. Radiotherapy planning was performed on a XiO system (Computerised Medical Systems) until July 2012, using a convolution-superposition algorithm with inhomogeneity corrections and according to International Commission on Radiation Units \& Measurements 50 guidelines. As of July 2012, radiotherapy planning was performed using RapidArc (Eclipse version 11.0), with a type B dose calculation algorithm (Acu$\operatorname{ros} X B-10.0)$.

\section{Endpoint}

Study endpoint was overall survival (OS) calculated from start of RT until the date of death or last follow-up. Survival information was retrieved from "Gemeentelijke Basis Administratie" (GBA), the decentralised population registration system in the Netherlands. A patient who was alive at the end of the study was considered right-censored.

\section{Blood-biomarker measurement}

Blood-biomarkers measurements of the development dataset can be found elsewhere [40]. Measurements of the validation cohort were performed in a certified laboratory, using commercially available kits, in order to easily translate the results into clinical practice. For each patient, 3 aliquots of $0.5 \mathrm{ml}$ of serum and 3 aliquots of $1.5 \mathrm{ml}$ of plasma were available, which had been collected before the first fraction of radiotherapy, processed using standard protocols and finally stored in the institutional biobank. Measurements in plasma were performed using enzyme-linked immunosorbent assays for OPN (Quantikine Human Osteopontin Immuno assay; R\&D Systems, Minneapolis, $M N$ ), CA-IX (Nuclea Diagnostics, Cambridge, MA), VEGF (R\&D Systems), and TLR4 (MyBioSource, San Diego, CA). Measurements in serum for IL-6 and IL-8 were determined on Immulite XPi 2000 with a solid phase, enzyme labelled, chemoluminescence sequential immunometric assay (Siemens Medical Solutions Diagnostics, LA), for CRP on Cobas 8000 using an immunoturbimetric assay (Roche Diagnostics, Mannheim, Germany), for CEA on Immulite XPi using a solid-phase, two-site sequential chemoluminescent immunometric assay (Siemens Medical Solutions Diagnostics), for CYFRA 21-1 on Kryptor with a sandwich immuno-fluorescent assay (Brahms, ThermoFisher, Hennigsdorf, Germany), for $\alpha 2 \mathrm{M}$ on BN ProSpec using immunonephelometric assays (Siemens Medical Solutions Diagnostics, LA, USA), and for sIL2R using an enzyme-linked immunosorbent assay (Diaclone, Basancon Cedex, France). 
The analytes OPN, CA-IX, VEGF and TLR4 were assayed in plasma in duplicate using a Victor multilabel counter (Perkinelmer, Turku, Finland), while all other biomarkers were measured in singletons.

\section{Descriptive statistics}

Comparison of the development and validation datasets distributions was performed using a $\chi 2$ test for categorical variables and a Student t-test for the continuous ones. Prior to this a variable transformation on the gross tumour volume (GTV) and blood biomarker measurements were performed, using a logarithmic approach. Missing data imputation was performed by Multivariate Imputation by Chained Equations (MICE). Correlation analysis was performed between clinical and blood-biomarker variables under analysis for both datasets.

\section{Model development}

A Cox proportional hazards model was developed and included validated clinical variables: gender, World Health Organization performance status (WHO-PS), forced expiratory volume in one second (FEV1s), number of positive lymph node stations identified in the diagnostic PET scans (lymph nodes) and GTV, defined as the sum of the GTV of the primary tumour and metastatic lymph nodes [41]. A least absolute shrinkage and selection operator (LASSO) method was applied for feature selection of the bloodbiomarkers, entered as continuous variables into the model. LASSO selects variables correlated to the measured outcome by shrinking coefficients weights, down to zero for the ones not correlated to outcome [42]. Regression coefficients were estimated, using the whole dataset, hazard ratios (HR) and confidence intervals $(\mathrm{Cl})$ were calculated. Schoenfeld residuals were used to check the proportional hazards assumption. Discrimination, reflecting a correct ordering of the relative predictions with respect to true outcomes (i.e. overall survival) for individuals, and model's ability to distinguish between individuals who experience the outcome from those who remained event free, was determined by the Harrell's concordance-index (c-index). This ranges from 0.5 , no discrimination (no greater than the chance expectation) to 1 indicating perfect discrimination.

\section{External Model validation}

Calibration of the model, which refers to the agreement between observed and predicted probabilities, was visually assessed (further details in the appendix). Discrimination ability of the model was also determined by the Harrell's c-index in an independent dataset. 


\section{Model updating}

The prognostic index (PI) was computed by multiplying the covariates with the estimated coefficients on the validation dataset: $P I=\sum_{i} \beta_{i} x_{i}$. The validated model was extended by inputting the PI (recalibrated if necessary) as a fixed variable and further selecting among new proposed blood-biomarkers. Performance was evaluated by means of an internal 10-fold CV c-index (further details in the appendix). A log likelihood test of model fit was conducted between the validated and extended model.

All statistical methods were performed in $\mathrm{R}$ (version 2.15.2), using the libraries survcomp, survival, rms, glmnet and corrplot [43].

\section{RESULTS}

Upon analysis, 161 and 132 patients in the derivation and validation cohorts, respectively, had died, and median follow-up time was 8.2 (95\% Cl: $7.3-9.3$ ) and 3.6 years (95\% Cl: 3.2 - 4.6). Kaplan Meier plots of the overall survival for both datasets are shown in Figure 1. Patient characteristics and blood biomarker measurements are shown in Table 1 and Table 2, including the missing measurements count. Homogeneity tests on the blood-biomarkers measures reflect the small differences that could be observed between development and validation datasets, for which a non-significant $\mathrm{p}$ value could be demonstrated. The exception to this lies on IL-8, that was significantly higher in the validation dataset, with, however, a non-drastically higher median. The highest Pearson correlation was 0.61 between IL- 6 and CRP in the development dataset $(r=0.72$ in the validation dataset). Correlation between clinical features and bloodbiomarkers was lower than 0.5 for all comparisons, demonstrating that these variables provide complementary information (Figure 2). Radiotherapy administered doses according to regimen up to August 2005 had an HR=0.17 ( $p<0.01)$, while the one afterwards had an $H R=0.90(p=0.52)$. Concurrent scheme presented with an $H R=0.62$ $(p=0.03)$. Administered dose had a risk of $0.98(p=0.03)$. 


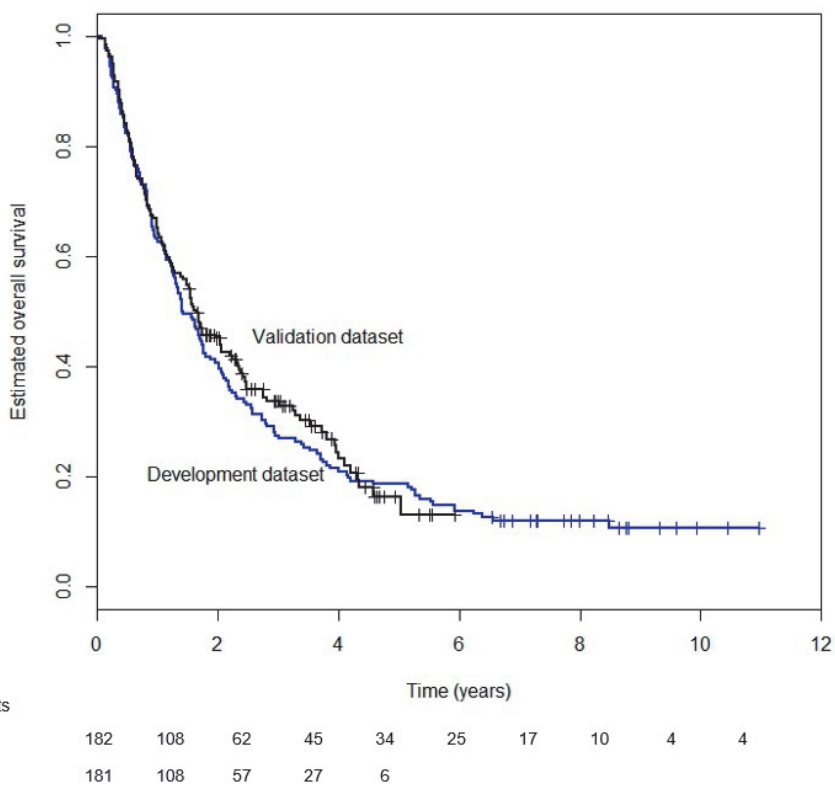

Figure 1 - Kaplan Meier plots for overall survival for development and validation datasets.

The model including solely clinical parameters achieved a performance with a c-index of 0.66 ( $p<0.01)$. The automatic feature selection algorithm used (LASSO), selected from the blood-biomarkers and extended the clinical model with OPN and Cyfra 21-1 (hazard ratios (HR) of 3.3 and 1.7), which led to a significant improvement in the performance up to 0.70 ( $p<0.01$; Table 3 ). Starting point for automatic feature selection methodology includes simultaneous consideration of all 8 biomarkers together with previously developed clinical model. Then, lower priority is assigned to features less associated with outcome, and therefore removed from the final model. Finally, features associated with survival outcome and not mutually correlated, were retained for the final model. The model including solely clinical parameters achieved a performance of 0.66 . The automatic feature selection algorithm used (LASSO), selected from the blood-biomarkers and extended the clinical model with OPN and Cyfra 21-1 (hazard ratios (HR) of 3.3 and 1.7), which led to a significant increase in the performance up to $0.70(p<0.01$; Table 3$)$.

As a side remark, most of the analysed blood biomarkers presented with a univariable significance correlation to overall survival (results in the online appendix), with the exception of CA-IX and IL-8. In addition, features included in final model presented with the most prominent HR of 7.2 (OPN) and 2.38 (Cyfra 21-1).

External validation of the clinical model yielded a c-index of 0.62 while the extended one improved to 0.66 . Despite the lower performance of the external validation, the addition of blood-biomarkers again showed an improvement in the performance. A nomogram based on this model is presented in the online appendix of this manuscript. 
In an exploratory exercise, the validated model was extended with $\alpha 2 \mathrm{M}$, sIL2 $\mathrm{r}$, and VEGF, and presented a c-index of 0.67 ( $p<0.01 ; 10$-fold CV c-index $=0.66$, Table 4). The validated model, as in Table 3 , is represented by its prognostic index (PI) in a new automatic feature selection routine, that extends it to include the mentioned bloodbiomarkers. Adding these biomarkers resulted in a better fitted model ( $p=0.01$; likelihood ratio test), and particularly $\alpha 2 \mathrm{M}$ and sIL2r were significantly associated with survival with HR of 4.6 and 3.1. Further details on how this extension is performed are descripted in the appendix, including model's recalibration.
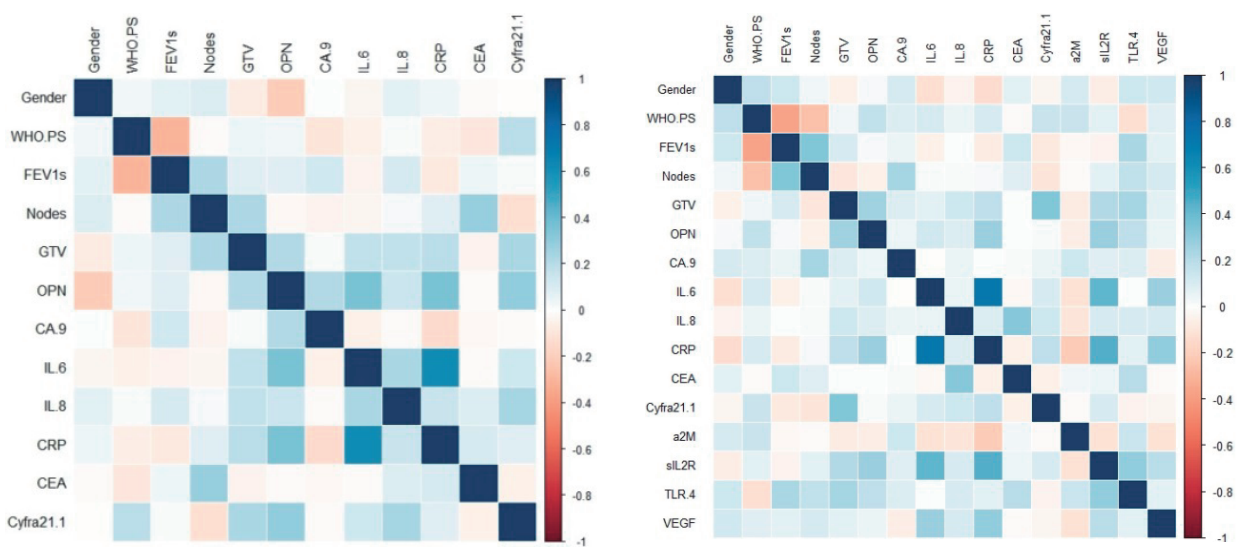

Figure 2 - Heat maps illustrating Pearson correlation between clinical features and blood biomarkers in the derivation (left) and validation (right) datasets. 


\section{CHAPTER 8}

Table 1 - Demographic information of the study population. Comparison between datasets distribution was performed after missing data imputation, with a $\chi^{2}$ test for categorical variables and a Student t-test for the continuous ones $(*)$.

\begin{tabular}{|c|c|c|c|}
\hline & Derivation dataset $(n=182)$ & Validation dataset $(n=181)$ & $p$-value* \\
\hline Age & & & 0.40 \\
\hline Range (median) & $42-87(69)$ & $44-88(68)$ & \\
\hline Mean \pm SD & $67.2 \pm 10.5$ & $68 \pm 9.3$ & \\
\hline Gender & & & 0.09 \\
\hline Male & $139(76.4 \%)$ & $123(68 \%)$ & \\
\hline Female & $43(23.6 \%)$ & $58(32 \%)$ & \\
\hline Stage & & & 0.06 \\
\hline 1 & $33(18.1 \%)$ & $20(11 \%)$ & \\
\hline$\|$ & $14(7.7 \%)$ & 25 (13.8\%) & \\
\hline IIla & $48(26.4 \%)$ & 49 (27.1\%) & \\
\hline$\| l \mathrm{~b}$ & 87 (47.8\%) & 85 (47\%) & \\
\hline Unknown (M0) & & $2(1.1 \%)$ & \\
\hline Histology & & & $<0.01$ \\
\hline Adenocarcinoma & $26(14.3 \%)$ & $39(21.5 \%)$ & \\
\hline Squamous cell & 49 (26.9\%) & $68(37.6 \%)$ & \\
\hline NOS & 91 (50\%) & 66 (36.5\%) & \\
\hline Unknown & $16(8.8 \%)$ & $8(4.4 \%)$ & \\
\hline WHO-PS & & & 0.43 \\
\hline 0 & 47 (25.8\%) & 48 (26.5\%) & \\
\hline 1 & $81(44.5 \%)$ & 93 (51.4\%) & \\
\hline$\geq 2$ & $17(9.3 \%)$ & $28(15.5 \%)$ & \\
\hline Unknown & 37 & $12(6.6 \%)$ & \\
\hline FEV 1s & & & 0.90 \\
\hline Range (median) & $33-124(76)$ & $25-136(76)$ & \\
\hline Mean \pm SD & $74 \pm 23.3$ & $74 \pm 24$ & \\
\hline Unknown & 14 & & \\
\hline Lymph nodes & & & 0.02 \\
\hline 0 & $73(40.1 \%)$ & 45 (24.9\%) & \\
\hline 1 & $26(14.3 \%)$ & $28(15.5 \%)$ & \\
\hline 2 & $32(17.6 \%)$ & 34 (18.8\%) & \\
\hline 3 & $20(11 \%)$ & $25(13.8 \%)$ & \\
\hline$\geq 4$ & $31(17 \%)$ & 49 (27\%) & \\
\hline $\operatorname{GTV}\left(\mathrm{cm}^{3}\right)$ & & & 0.12 \\
\hline Range (median) & $0.84-674.4(63.5)$ & $6.15-1076(104)$ & \\
\hline Mean \pm SD & $89.8 \pm 97.7$ & $128 \pm 142$ & \\
\hline Unknown & 3 & 8 & \\
\hline \multicolumn{4}{|c|}{ Radiotherapy (Range (mean $\pm S D$ ), in Gy) } \\
\hline \multicolumn{4}{|c|}{ Standard protocol } \\
\hline Before August 2005 & $58-70(62.4 \pm 4.3)$ & - & \\
\hline After August 2005 & $50.40-79.2(64.2 \pm 9.8)$ & $52.2-79.2(68.9 \pm 11.9)$ & $<0.01$ \\
\hline \multicolumn{4}{|l|}{ Concurrent scheme } \\
\hline First Dose & $45-51(46.9 \pm 3.5)$ & $45(45.9 \pm 6.1)$ & \\
\hline
\end{tabular}


Acronyms: NOS - not otherwise specified, also includes patients categorized as "large cell"; WHO-PS - world health organization performance status; FEV 1s - forced expiratory volume in 1 second; Lymph nodes refer to the PET positive stations identified; GTV - gross tumour volume (GTV = volume of primary tumour + volume of PET positive lymph node stations)

* Significance of the homogeneity tests between datasets distributions

Table 2 - Distribution of the blood-biomarkers after data imputation: range (median); mean \pm standard deviation. Biomarkers were not measured case there was not enough material. New biomarkers were only available in the latest measurements solely performed in the validation dataset. Comparison between datasets distribution of markers was conducted with a Student t-test $\left({ }^{*}\right)$.

\begin{tabular}{|c|c|c|c|c|c|}
\hline Blood Biomarker & $\begin{array}{c}\text { Development } \\
\text { Dataset }(n=182)\end{array}$ & $\begin{array}{l}\text { Not } \\
\text { measured }\end{array}$ & $\begin{array}{l}\text { Validation Dataset } \\
\qquad(\mathrm{n}=181)\end{array}$ & $\begin{array}{l}\text { Not } \\
\text { measured }\end{array}$ & $p$-value* \\
\hline \multicolumn{6}{|l|}{ Hypoxia } \\
\hline Osteopontin (OPN) & $\begin{array}{c}40-304 \text { (99) } \\
111 \pm 46\end{array}$ & 3 & $\begin{array}{c}16-1802(140) \\
128 \pm 137\end{array}$ & 1 & 0.13 \\
\hline $\begin{array}{l}\text { Carbonic Anhydrase IX (CA- } \\
\text { IX) }\end{array}$ & $\begin{array}{c}59-2477(221) \\
337 \pm 346\end{array}$ & 2 & $\begin{array}{c}48-8508(254) \\
402 \pm 696\end{array}$ & 1 & 0.26 \\
\hline \multicolumn{6}{|l|}{ Inflammation } \\
\hline Interleukin 6 (IL-6) & $\begin{array}{l}1.1-86(7.2) \\
10 \pm 11\end{array}$ & 4 & $\begin{array}{c}1.8-462(6.4) \\
14 \pm 39\end{array}$ & 0 & 0.26 \\
\hline Interleukin 8 (IL-8) & $\begin{array}{c}2.3-91(11) \\
14 \pm 13\end{array}$ & 5 & $\begin{array}{c}4.7-316(13) \\
20 \pm 28\end{array}$ & 0 & 0.01 \\
\hline C-reactive protein (CRP) & $\begin{array}{c}1-315(12) \\
26 \pm 41\end{array}$ & 16 & $\begin{array}{c}0.9-258(8.8) \\
24 \pm 39\end{array}$ & 0 & 0.55 \\
\hline \multicolumn{6}{|l|}{ Tumour load } \\
\hline $\begin{array}{l}\text { Carcinoembryonic antigen } \\
\text { (CEA) }\end{array}$ & $\begin{array}{c}0.8-1806(4.3) \\
32 \pm 152\end{array}$ & 9 & $\begin{array}{l}0.7-1068(5.4) \\
33 \pm 115\end{array}$ & 0 & 0.91 \\
\hline $\begin{array}{l}\text { Cytokeratin fragment (CYFRA } \\
\text { 21-1) }\end{array}$ & $\begin{array}{c}0.2-49(1.7) \\
3.7 \pm 6.4\end{array}$ & 5 & $\begin{array}{c}0.1-119(2.0) \\
6.2 \pm 16\end{array}$ & 1 & 0.05 \\
\hline \multicolumn{6}{|l|}{ New biomarkers } \\
\hline $\begin{array}{l}\text { Alpha-2-Macroglobulin } \\
(\alpha 2 \mathrm{M})\end{array}$ & & & $\begin{array}{c}1.1-5.2(2.3) \\
2.4 \pm 0.8\end{array}$ & 5 & \\
\hline Serum IL-2 receptor (sIL2R) & & & $\begin{array}{c}1121-20000 \\
(5926) \\
6129 \pm 2907\end{array}$ & 0 & \\
\hline Toll-like receptor 4 (TLR4) & & & $\begin{array}{l}1.4-30(6.4) \\
7.5 \pm 4.6\end{array}$ & 1 & \\
\hline $\begin{array}{l}\text { Vascular Endothelial Growth } \\
\text { Factor (VEGF) }\end{array}$ & & & $\begin{array}{c}18-505(84) \\
112 \pm 94\end{array}$ & 1 & \\
\hline
\end{tabular}

Acronyms: SD - Standard Deviation

* Significance of the homogeneity tests between datasets distributions 


\section{CHAPTER 8}

Table 3 - Multivariable Cox PH regression of the clinical variables and of clinical variables and bloodbiomarkers fitted on the derivation dataset. Performance of the model expressed in terms of internal and external validation $(*)$ by Harrell's c-index.

\begin{tabular}{|c|c|c|c|c|c|c|c|c|}
\hline Feature & Hazard Ratio & p-value & $95 \% \mathrm{Cl} \mathrm{HR}$ & c-index & $\begin{array}{l}\text { Hazard } \\
\text { Ratio }\end{array}$ & $\mathrm{p}$-value & $95 \% \mathrm{Cl} \mathrm{HR}$ & c-index \\
\hline \multicolumn{9}{|l|}{ Gender } \\
\hline Male & \multicolumn{4}{|l|}{ Reference } & \multicolumn{3}{|c|}{ Reference } & \\
\hline Female & 0.50 & $<0.01$ & $0.33-0.75$ & & 0.54 & $<0.01$ & $0.36-0.82$ & \\
\hline \multicolumn{9}{|l|}{ WHO-PS } \\
\hline 0 & \multicolumn{4}{|l|}{ Reference } & \multicolumn{3}{|c|}{ Reference } & \multirow{13}{*}{$\begin{array}{c}0.70 \\
0.66^{*}\end{array}$} \\
\hline 1 & 1.30 & 0.01 & $0.90-1.88$ & \multirow{5}{*}{$\begin{array}{c}0.66 \\
0.62 *\end{array}$} & 1.20 & 0.01 & $0.82-1.74$ & \\
\hline$\geq 2$ & 2.65 & & $1.57-4.45$ & & 2.09 & & $1.22-3.58$ & \\
\hline FEV 1s & 1.00 & 0.88 & $0.99-1.01$ & & 1.00 & 0.45 & $0.99-1.00$ & \\
\hline Lymph nodes & & & & & & & & \\
\hline 0 & \multicolumn{3}{|l|}{ Reference } & & \multicolumn{3}{|c|}{ Reference } & \\
\hline 1 & 0.63 & 0.14 & $0.37-1.06$ & & 0.63 & 0.14 & $0.37-1.07$ & \\
\hline 2 & 1.03 & & $0.63-1.66$ & & 1.12 & & $0.69-1.83$ & \\
\hline 3 & 0.59 & & $0.34-1.05$ & & 0.62 & & $0.35-1.09$ & \\
\hline 4 & 0.91 & & $0.57-1.46$ & & 1.10 & & $0.68-1.80$ & \\
\hline $\ln (G T V)\left(\mathrm{cm}^{3}\right)$ & 1.41 & $<0.01$ & $1.21-1.64$ & & 1.28 & $<0.01$ & $1.09-1.50$ & \\
\hline OPN & & & & & 3.31 & 0.01 & $1.31-8.38$ & \\
\hline Cyfra 21-1 & & & & & 1.71 & 0.01 & $1.18-2.50$ & \\
\hline
\end{tabular}

A logarithmic transformation was performed on the blood-biomarkers measurements prior to analysis; Acronyms: $\mathrm{Cl}$ - Confidence Interval; HR - Hazard Ratio; HR - hazard ratio; WHO-PS - world health organization performance status; FEV 1s - forced expiratory volume in 1 second; Lymph nodes refer to the PET positive stations identified; GTV - gross tumour volume (GTV = volume of primary tumour + volume of PET positive lymph node stations); OPN - Osteopontin; Cyfra 21-1 - cytokeratin fragment 21-1; *External validation

Table 4 - Multivariable Cox PH regression of the clinical variables and blood-biomarkers fitted on the validation dataset, after a feature selection made by LASSO from newly analysed blood-biomarkers. Performance of the model is expressed in terms of internal c-index, corrected for optimism by a 10 -fold CV $(* *)$.

\begin{tabular}{llllc}
\hline Feature & Hazard Ratio & p-value & $95 \% \mathrm{Cl} \mathrm{HR}$ & c-index \\
\hline Calibrated PI* & 2.44 & $<0.01$ & $1.69-3.51$ & 0.67 \\
a2M & 4.62 & $<0.01$ & $1.31-16.3$ & $0.66^{* *}$ \\
SIL2R & 3.15 & $<0.01$ & $1.08-9.21$ & \\
VEGF & 1.37 & 0.28 & $0.78-2.43$ & \\
\hline
\end{tabular}

A logarithmic transformation was performed on the blood-biomarkers measurements prior to analysis; Acronyms: $\alpha 2 M$ - alpha-2-macroglobulin; sIL2 $r$ - serum interleukin-2 receptor; VEGF - vascular endothelial growth factor; * Validated model in TABLE 3 entered as a variable - the calibrated prognostic index (PI); ** Internal cindex corrected for optimism by a 10 -fold CV 


\section{DISCUSSION}

Prognostic modelling of NSCLC is becoming an important element of the disease management. Several sources of information now available make it an emerging and constantly changing field, however the choice for non-invasive techniques is preferred in order to diminish the burden for patients. The use of blood-biomarker measurements appears to be a non-invasive, fast and promising source of extra information, reflecting dissimilarities of the tumour microenvironment that has been shown to be associated with disease prognosis and response to treatment $[44,45]$.

We demonstrated and validated in two large cohorts of NSCLC patients the added value of blood-biomarkers related to hypoxia (OPN) and tumour load (Cyfra 21-1), reflected by a statistically significant improvement in the performance of a clinical model after their inclusion. A priori selection of blood-biomarkers was based on a literature search and evidence of their prognostic value for NSCLC [11-25]. Hypoxia affects malignant progression by increasing the tumour's metastatic ability and diminishing the apoptotic potential, and also the response to therapy, by decreasing the effects of anticancer therapies including (chemo)radiotherapy [44]. In addition, imaging studies with hypoxia-specific markers have shown that hypoxia is a prevalent effect in NSCLC [46]. The hypoxia marker OPN, has also been associated with tumour aggressiveness and metastatic potential, accompanying a poor prognosis in NSCLC, confirmed rather strongly in our study $(H R=3.3)[19,20]$. Tumour load is often associated with disease development and prognosis [41, 47]. Cyfra 21-1, a marker associated with tumour load, was previously identified as a prognostic factor for NSCLC, which was also confirmed in our analysis [48].

We have already investigated the prognostic potential of blood-biomarkers using a support vector machine (SVM) for model development. We then extended a validated 2-year survival clinical model with blood-biomarkers related to inflammation (IL-6) and tumour load (CEA). Performance expressed as the area under the curve (AUC) improved from 0.72 (solely clinical parameters) to $0.81[40,41]$. However, the limited number of patients included in the study made it difficult to draw definitive conclusions. Moreover, SVM is less suited to a time-to-event analysis, as it requires dichotomous outcome. Therefore, we re-analysed the data, applying Cox regression. In the newly fitted clinical model, moderately good performance $(0.66$, external $=0.62)$ could be achieved with female gender which is associated with a better prognosis, given the lower risk $(H R=0.50)$, while WHO-PS $\geq 2(H R=2.65)$ and larger $G T V(H R=1.41)$ are worse prognostic factors.

The validated model was extended to include $\alpha 2 \mathrm{M}$, sIL2r, and VEGF. Despite the comparable c-index $(0.67 ; 10$-fold $\mathrm{CV}=0.66)$, most importantly it showed that higher concentrations of these new markers are associated with a worse prognosis, particularly $\alpha 2 \mathrm{M}$ and sIL2r with HRs of 4.6 and 3.1. Previous studies showed $\alpha 2 \mathrm{M}$ as a top candidate for radiation pneumonitis [31]. We further confirmed its prognostic potential for NSCLC. IL-2 
was already identified as an independent prognostic marker in patients with advanced NSCLC [49]. Its cell surface receptor, a soluble form of IL-2 receptor (sIL2r) is released into the bloodstream and involved in the regulation of IL-2. High sIL2 $r$ levels were associated with shorter survival in an advanced stage $[50,51]$. Elevated levels of sIL2 $r$ may lead to a decreased cellular response to IL-2, reinforcing the importance of measuring this marker for patients receiving IL-2 immunotherapy [51-53]. VEGF, also included in the final model with a less expressive HR of 1.4, is a common angiogenesis factor for a variety of solid tumours, including NSCLC [54]. It has been shown previously to be an independent prognostic factor, which was not however confirmed by our data [14].

An advantage of our study is the large number of patients available, making the derived message a more solid one. Conversely, this study had some limitations. First, it was impossible to validate our latest findings using the newer biomarkers as these were only measured in the validation dataset. This should be thoroughly considered in the future, by gathering data from external institute(s). The heterogeneity of our dataset, including more recently treated patients following concurrent schemes in the validation dataset, the different types and scheduling of chemotherapy, and the different laboratories involved in the biomarkers measurements may provide us to a better understanding of the derived calibration slope, which is below 1 [55]. However, the c-index achieved in the context of a prognostic analysis must not be disregarded, particularly when compared with the limited capacity of humans to infer better prognosis from the same sources of information [7]. Also due to its great heterogeneity, it is not possible with this cohort to assess stage-specific treatment variables. We are however able to deliver a set of prognostic factors, independent of dissimilar types of treatment or disease stage, but spanning a larger cohort of heterogeneous patients. This model could be improved by including other sources of information, for example imaging (Radiomics), as the relevant prognostic value of sophisticated image analysis has been shown, to be investigated in the future $[8,9]$. One last point that we would like to emphasise is the increasing number of PET tracers currently available and the ability to obtain extra information, in particular relating to hypoxia $\left({ }^{18} \mathrm{~F}\right.$-FMISO, ${ }^{18} \mathrm{~F}$-FAZA and ${ }^{18} \mathrm{~F}$ HX4), tumour proliferation (18F-fluorothymidine), and assessment of epidermal growth factor receptor (EGFR) [56-58]. The potential use of blood-biomarkers to predict which imaging tracer is best suited to an individual patient, would convert prognostic markers into predictive information and increase the cost effectiveness of imaging procedures, while diminishing patient burden from extensive diagnostic imaging procedures.

In conclusion, we improved a clinical model by the inclusion of blood-biomarkers related to hypoxia and tumour load. This improvement was validated externally, which reinforces its potential relevance for shared decision-making. An extended model demonstrated that higher concentrations of the inflammation marker $\alpha 2 \mathrm{M}$ and immunological marker sIL2r have strong negative prognostic value for NSCLC, which requires extra validation. Data is publicly available at www.cancerdata.org (http://dx.doi.org/10.17195/candat. 2016.04.1) and the developed models can be found at www.predictcancer.org. 


\section{ACKNOWLEDGEMENTS}

Authors acknowledge financial support from the QulC-ConCePT project, which is partly funded by EFPIA companies and the Innovative Medicine Initiative Joint Undertaking (IMIJU) under Grant Agreement No. 115151. This research is also supported by the Dutch technology Foundation STW (grant $n^{\circ} 10696$ DuCAT), which is the applied science division of NWO, and the Technology Programme of the Ministry of Economic Affairs. Authors also acknowledge financial support from the CTMM framework (AIRFORCE project, grant 030-103), EU 7th framework programme (EURECA, ARTFORCE - $\mathrm{n}^{\circ}$ 257144, REQUITE - n 601826), SME Phase 2 (EU proposal 673780 - RAIL), Kankeronderzoekfonds Limburg from the Health Foundation Limburg and the Dutch Cancer Society (KWF UM 2008-4210, KWF UM 2009-4454). 


\section{REFERENCES}

[1] Torre LA, Siegel RL, Ward EM, Jemal A. Global Cancer Incidence and Mortality Rates and Trends-An Update. Cancer Epidemiol Biomarkers Prev. 2016;25 (1):16-25.

[2] Dingemans AM, Bootsma G, van Baardwijk A, Reymen B, Wanders R, Brans B, et al. A phase I study of concurrent individualized, isotoxic accelerated radiotherapy and cisplatin-vinorelbine-cetuximab in patients with stage III non-small-cell lung cancer. J Thorac Oncol. 2014;9:710-6.

[3] De Ruysscher D, van Baardwijk A, Steevens J, Botterweck A, Bosmans G, Reymen B, et al. Individualised isotoxic accelerated radiotherapy and chemotherapy are associated with improved long-term survival of patients with stage III NSCLC: a prospective population-based study. Radiother Oncol. 2012;102:228-33.

[4] De Ruysscher D, Wanders R, van Baardwijk A, Dingemans AM, Reymen B, Houben R, et al. Radical treatment of non-small-cell lung cancer patients with synchronous oligometastases: long-term results of a prospective phase II trial (Nct01282450). J Thorac Oncol. 2012;7:1547-55.

[5] Lambin P, Roelofs E Fau - Reymen B, Reymen B Fau - Velazquez ER, Velazquez Er Fau - Buijsen J, Buijsen J Fau - Zegers CML, Zegers Cm Fau - Carvalho S, et al. 'Rapid Learning health care in oncology' - an approach towards decision support systems enabling customised radiotherapy'. Radiother Oncol. 2013;109(1):159-64.

[6] Lambin P, van Stiphout Rg Fau - Starmans MHW, Starmans Mh Fau - Rios-Velazquez E, Rios-Velazquez E Fau - Nalbantov G, Nalbantov G Fau - Aerts HJWL, Aerts Hj Fau - Roelofs E, et al. Predicting outcomes in radiation oncology--multifactorial decision support systems. Nature reviews Clinical oncology. 2013;10(1):27-40.

[7] Oberije C, Nalbantov G, Dekker A, Boersma L, Borger J, Reymen B, et al. A prospective study comparing the predictions of doctors versus models for treatment outcome of lung cancer patients: a step toward individualized care and shared decision making. Radiother Oncol. 2014;112:37-43.

[8] Lambin P, Rios-Velazquez E, Leijenaar R, Carvalho S, van Stiphout RG, Granton P, et al. Radiomics: extracting more information from medical images using advanced feature analysis. Eur J Cancer. 2012;48:441-6.

[9] Aerts HJ, Velazquez ER, Leijenaar RT, Parmar C, Grossmann P, Carvalho S, et al. Decoding tumour phenotype by noninvasive imaging using a quantitative radiomics approach. Nat Commun. 2014;5:4006.

[10] Sozzi G, Boeri M. Potential biomarkers for lung cancer screening. Transl Lung Cancer Res. 2014;3(3):139-48.

[11] Donati V, Boldrini L, Dell'Omodarme M, Prati MC, Faviana P, Camacci T, et al. Osteopontin expression and prognostic significance in non-small cell lung cancer. Clin Cancer Res. 2005;11:6459-65.

[12] Fiala O, Pesek M, Finek J, Topolcan O, Racek J, Minarik M, et al. High serum level of C-reactive protein is associated with worse outcome of patients with advanced-stage NSCLC treated with erlotinib. Tumour Biol. 2015;36(12):9215-22.

[13] Mendes F, Antunes C, Abrantes AM, Goncalves AC, Nobre-Gois I, Sarmento AB, et al. Lung cancer: the immune system and radiation. Br J Biomed Sci. 2015;72:78-84.

[14] Ostheimer C, Bache M, Guttler A, Kotzsch M, Vordermark D. A pilot study on potential plasma hypoxia markers in the radiotherapy of non-small cell lung cancer. Osteopontin, carbonic anhydrase IX and vascular endothelial growth factor. Strahlenther Onkol. 2014;190:276-82.

[15] Rud AK, Boye K, Oijordsbakken M, Lund-Iversen M, Halvorsen AR, Solberg SK, et al. Osteopontin is a prognostic biomarker in non-small cell lung cancer. BMC Cancer. 2013;13:540.

[16] Sanmamed MF, Carranza-Rua O, Alfaro C, Onate C, Martin-Algarra S, Perez G, et al. Serum interleukin-8 reflects tumour burden and treatment response across malignancies of multiple tissue origins. Clin Cancer Res. 2014;20:5697-707.

[17] Wang J, Yi Y, Li B, Wang Z, Sun H, Zhang P, et al. CYFRA21-1 can predict the sensitivity to chemoradiotherapy of non-small-cell lung carcinoma. Biomarkers. 2010;15:594-601.

[18] Wang J, Zhang N, Li B, Wang Z, Sun H, Yi Y, et al. Decline of serum CYFRA21-1 during chemoradiotherapy of NSCLC: a probable predictive factor for tumour response. Tumour Biol. 2011;32:689-95. 
[19] Zou XL, Wang C, Liu KE, Nie W, Ding ZY. Prognostic significance of osteopontin expression in non-smallcell lung cancer: A meta-analysis. Mol Clin Oncol. 2015;3:633-8.

[20] Liu Y, Gu X, Lin Q, Tian T, Shao L, Yuan C, et al. Prognostic significance of osteopontin in patients with non-small cell lung cancer: results from a meta-analysis. International journal of clinical and experimental medicine. 2015;8:12765-73.

[21] Jing X, Huang C, Zhou H, Li C, Fan L, Chen J, et al. Association between serum C-reactive protein value and prognosis of patients with non-small cell lung cancer: a meta-analysis. International journal of clinical and experimental medicine. 2015;8:10633-9.

[22] Ilie M, Mazure NM, Hofman V, Ammadi RE, Ortholan C, Bonnetaud C, et al. High levels of carbonic anhydrase IX in tumour tissue and plasma are biomarkers of poor prognostic in patients with non-small cell lung cancer. British journal of cancer. 2010;102:1627-35.

[23] Gomes M, Coelho A, Araujo A, Azevedo A, Teixeira AL, Catarino R, et al. IL-6 polymorphism in non-small cell lung cancer: a prognostic value? Tumour Biol. 2015;36:3679-84.

[24] Liao C, Yu Z, Guo W, Liu Q, Wu Y, Li Y, et al. Prognostic value of circulating inflammatory factors in nonsmall cell lung cancer: a systematic review and meta-analysis. Cancer biomarkers : section A of Disease markers. 2014;14:469-81.

[25] Zhang ZH, Han YW, Liang H, Wang LM. Prognostic value of serum CYFRA21-1 and CEA for non-small-cell lung cancer. Cancer medicine. 2015;4:1633-8.

[26] Bien E, Balcerska A. Serum soluble interleukin 2 receptor alpha in human cancer of adults and children: a review. Biomarkers. 2008;13:1-26.

[27] De Vita F, Turitto G, di Grazia M, Frattolillo A, Catalano G. Analysis of interleukin-2/interleukin-2 receptor system in advanced non-small-cell lung cancer. Tumouri. 1998;84:33-8.

[28] Fu HY, Li C, Yang W, Gai XD, Jia T, Lei YM, et al. FOXP3 and TLR4 protein expression are correlated in nonsmall cell lung cancer: implications for tumour progression and escape. Acta Histochem. 2013;115:151-7.

[29] Kawashima O, Kamiyoshihara M, Sakata S, Endo K, Saito R, Morishita Y. The clinicopathological significance of preoperative serum-soluble interleukin-2 receptor concentrations in operable non-small-cell lung cancer patients. Ann Surg Oncol. 2000;7:239-45.

[30] Naumnik W, Chyczewska E. The clinical significance of serum soluble interleukin 2 receptor (sIL-2R) concentration in lung cancer. Folia Histochem Cytobiol. 2001;39 Suppl 2:185-6.

[31] Oh JH, Craft JM, Townsend R, Deasy JO, Bradley JD, El Naqa I. A bioinformatics approach for biomarker identification in radiation-induced lung inflammation from limited proteomics data. J Proteome Res. 2011;10:1406-15.

[32] Rades D, Setter C, Dunst J, Dahl O, Schild SE, Noack F. Prognostic impact of VEGF and VEGF receptor 1 (FLT1) expression in patients irradiated for stage II/III non-small cell lung cancer (NSCLC). Strahlenther Onkol. 2010;186:307-14.

[33] Samara KD, Antoniou KM, Karagiannis K, Margaritopoulos G, Lasithiotaki I, Koutala E, et al. Expression profiles of Toll-like receptors in non-small cell lung cancer and idiopathic pulmonary fibrosis. Int J Oncol. 2012;40:1397-404.

[34] Carrizosa DR, Gold KA. New strategies in immunotherapy for non-small cell lung cancer. Transl Lung Cancer Res. 2015;4:553-9.

[35] Simone CB, 2nd, Burri SH, Heinzerling JH. Novel radiotherapy approaches for lung cancer: combining radiation therapy with targeted and immunotherapies. Transl Lung Cancer Res. 2015;4:545-52.

[36] van Baardwijk A, Wanders S Fau - Boersma L, Boersma L Fau - Borger J, Borger J Fau - Ollers M, Ollers M Fau - Dingemans A-MC, Dingemans Am Fau - Bootsma G, et al. Mature results of an individualized radiation dose prescription study based on normal tissue constraints in stages I to III non-small-cell lung cancer. J Clin Oncol. 2010;28(8):1380-6.

[37] Oncology MR. Concurrent chemo-radiation for NSCLC to an individualized MLD, NLM identifier: NCT005 72325. 2007.

[38] van Elmpt W, De Ruysscher D Fau - van der Salm A, van der Salm A Fau - Lakeman A, Lakeman A Fau van der Stoep J, van der Stoep J Fau - Emans D, Emans D Fau - Damen E, et al. The PET-boost randomised phase II dose-escalation trial in non-small cell lung cancer. Radiother Oncol. 2012;104(1):67-71. 
[39] Senan S, De Ruysscher D, Giraud P, Mirimanoff R, Budach V. Literature-based recommendations for treatment planning and execution in high-dose radiotherapy for lung cancer. Radiother Oncol. 2004;71:139-46.

[40] Dehing-Oberije C, Aerts H, Yu S, De Ruysscher D, Menheere P, Hilvo M, et al. Development and validation of a prognostic model using blood biomarker information for prediction of survival of non-small-cell lung cancer patients treated with combined chemotherapy and radiation or radiotherapy alone (NCT00181519, NCT00573040, and NCT00572325). Int J Radiat Oncol Biol Phys. 2011;81:360-8.

[41] Dehing-Oberije C, Yu S, De Ruysscher D, Meersschout S, Van Beek K, Lievens Y, et al. Development and external validation of prognostic model for 2-year survival of non-small-cell lung cancer patients treated with chemoradiotherapy. Int J Radiat Oncol Biol Phys. 2009;74:355-62.

[42] Tibshirani R. The lasso method for variable selection in the Cox model. Stat Med. 1997;16(4):385-95.

[43] Team RC. R: A language and environment for statistical computing. R Foundation for Statistical Computing. 2.15.2 ed2015.

[44] Graves EE, Maity A, Le QT. The Tumour Microenvironment in Non-Small Cell Lung Cancer. Semin Radiat Oncol. 2010;20:156-63.

[45] Quail D, Joyce J. Microenvironmental regulation of tumour progression and metastasis. Nat Med. 2013;19:1423-37.

[46] Zegers CM, van Elmpt W, Reymen B, Even AJ, Troost EG, Ollers MC, et al. In vivo quantification of hypoxic and metabolic status of NSCLC tumours using [18F]HX4 and [18F]FDG-PET/CT imaging. Clin Cancer Res. 2014;20:6389-97.

[47] Soliman M, Yaromina A Fau - Appold S, Appold S Fau - Zips D, Zips D Fau - Reiffenstuhl C, Reiffenstuhl C Fau - Schreiber A, Schreiber A Fau - Thames HD, et al. GTV differentially impacts locoregional control of non-small cell lung cancer (NSCLC) after different fractionation schedules: subgroup analysis of the prospective randomized CHARTWEL trial. Radiother Oncol. 2013;106(3):299-304.

[48] Xu Y, Xu L, Qiu M, Wang J, Zhou Q, Yin R. Prognostic value of serum cytokeratin 19 fragments (Cyfra 21-1) in patients with non-small cell lung cancer. Sci Rep. 2015;5:9444.

[49] Orditura M, Romano C, De Vita F, Galizia G, Lieto E, Infusino S, et al. Behaviour of interleukin-2 serum levels in advanced non-small-cell lung cancer patients: relationship with response to therapy and survival. Cancer Immunol Immunother. 2000;49:530-6.

[50] Brunetti G, Bossi A, Baiardi P, Jedrychowska I, Pozzi U, Bacchella L, et al. Soluble interleukin 2 receptor (sIL2R) in monitoring advanced lung cancer during chemotherapy. Lung Cancer. 1999;23:1-9.

[51] Witkowska AM. On the role of sIL-2R measurements in rheumatoid arthritis and cancers. Mediators Inflamm. 2005;2005:121-30.

[52] Zegers CM, Rekers NH, Quaden DH, Lieuwes NG, Yaromina A, Germeraad WT, et al. Radiotherapy combined with the immunocytokine L19-IL2 provides long-lasting antitumour effects. Clin Cancer Res. 2015;21(5):1151-60.

[53] van den Heuvel MM, Verheij M, Boshuizen R, Belderbos J, Dingemans AM, De Ruysscher D, et al. NHS-IL2 combined with radiotherapy: preclinical rationale and phase $\mathrm{Ib}$ trial results in metastatic non-small cell lung cancer following first-line chemotherapy. J Transl Med. 2015:13:32.

[54] Das M, Wakelee H. Targeting VEGF in lung cancer. Expert Opinion on Therapeutic Targets 2012;16:395-406.

[55] Auperin A, Le Pechoux C Fau - Rolland E, Rolland E Fau - Curran WJ, Curran Wj Fau - Furuse K, Furuse K Fau - Fournel P, Fournel P Fau - Belderbos J, et al. Meta-analysis of concomitant versus sequential radiochemotherapy in locally advanced non-small-cell lung cancer. J Clin Oncol. 2010;28(13):2181-90.

[56] Peeters SG, Zegers CM, Lieuwes NG, van Elmpt W, Eriksson J, van Dongen GA, et al. A comparative study of the hypoxia PET tracers [(1)(8)F]HX4, [(1)(8)F]FAZA, and [(1)(8)F]FMISO in a preclinical tumour model. Int J Radiat Oncol Biol Phys. 2015;91(2):351-9.

[57] Zander T, Scheffler M Fau - Nogova L, Nogova L Fau - Kobe C, Kobe C Fau - Engel-Riedel W, Engel-Riedel W Fau - Hellmich M, Hellmich M Fau - Papachristou I, et al. Early prediction of nonprogression in advanced non-small-cell lung cancer treated with erlotinib by using [(18)F]fluorodeoxyglucose and [(18)F]fluorothymidine positron emission tomography. J Clin Oncol. 2011;29(13):1701-8. 
[58] Mishani E, Abourbeh G Fau - Eiblmaier M, Eiblmaier M Fau - Anderson CJ, Anderson CJ. Imaging of EGFR and EGFR tyrosine kinase overexpression in tumours by nuclear medicine modalities. Curr Pharm Des. 2008;14(28):2983-98. 


\section{APPENDIX}

This appendix aims to provide extra information on the statistical analysis conducted. It also presents complementary information to the results section of main manuscript.

\section{Univariable analysis}

A univariable Cox proportional hazards regression was conducted for the clinical variables and blood biomarkers analysed in the development dataset. These results are shown in Table 1.

Table 1 - Univariable Cox proportional hazards regression of blood-biomarkers related to hypoxia, inflammation and tumour load. Univariable performance of each marker expressed in terms of Harrell's c-index.

\begin{tabular}{|c|c|c|c|c|}
\hline Feature & Hazard Ratio & $95 \% \mathrm{Cl} \mathrm{HR}$ & $p$-value & c-index \\
\hline \multicolumn{5}{|l|}{ Gender } \\
\hline Male & 1.98 & $1.33-2.94$ & $<0.01$ & 0.58 \\
\hline Female & 0.51 & $0.34-0.75$ & $<0.01$ & 0.58 \\
\hline \multicolumn{5}{|l|}{ WHO-PS } \\
\hline 0 & 0.74 & $0.53-1.04$ & 0.08 & 0.55 \\
\hline 1 & 1.01 & $0.74-1.37$ & 0.97 & 0.52 \\
\hline$\geq 2$ & 1.82 & $1.19-2.80$ & $<0.01$ & 0.53 \\
\hline FEV 1s & 0.99 & $0.99-1.01$ & 0.86 & 0.52 \\
\hline \multicolumn{5}{|l|}{ Lymph nodes } \\
\hline 0 & 1.09 & $0.80-1.48$ & 0.60 & 0.51 \\
\hline 1 & 0.61 & $0.37-0.99$ & 0.05 & 0.53 \\
\hline 2 & 1.26 & $0.82-1.91$ & 0.29 & 0.52 \\
\hline 3 & 0.85 & $0.50-1.42$ & 0.53 & 0.51 \\
\hline 4 & 1.22 & $0.81-1.85$ & 0.34 & 0.52 \\
\hline $\ln (G T V)\left(\mathrm{cm}^{3}\right)$ & 1.34 & $1.17-1.53$ & $<0.01$ & 0.63 \\
\hline \multicolumn{5}{|l|}{ Hypoxia } \\
\hline Osteopontin (OPN) & 7.17 & $3.09-16.6$ & $<0.01$ & 0.62 \\
\hline Carbonic Anhydrase IX (CA-IX) & 0.97 & $0.60-1.57$ & 0.90 & 0.50 \\
\hline \multicolumn{5}{|l|}{ Inflammation } \\
\hline Interleukin 6 (IL-6) & 1.98 & $1.31-2.99$ & $<0.01$ & 0.61 \\
\hline Interleukin 8 (IL-8) & 1.22 & $0.69-2.18$ & 0.50 & 0.55 \\
\hline C-reactive protein (CRP) & 1.93 & $1.45-2.59$ & $<0.01$ & 0.61 \\
\hline \multicolumn{5}{|l|}{ Tumour load } \\
\hline Carcinoembryonic Antigen (CEA) & 1.45 & $1.12-1.87$ & $<0.01$ & 0.57 \\
\hline Cytokeratin fragment 21-1 (Cyfra 21-1) & 2.38 & $1.75-3.24$ & $<0.01$ & 0.63 \\
\hline
\end{tabular}

Abbreviations: $\mathrm{Cl}$ - confidence interval; HR - Hazard ratio 


\section{Multivariable analysis}

A Cox proportional hazards regression model was developed and selected from blood biomarkers representative of hypoxia, inflammation and tumour load, in addition to validated clinical variables (gender, World Health Organization performance status (WHO-PS), forced expiratory volume in one second (FEV1s), number of positive lymph node stations identified in the diagnostic PET scans (lymph nodes) and GTV, defined as the sum of the GTV of the primary tumour and metastatic lymph nodes [1]). A least absolute shrinkage and selection operator (LASSO) method was applied for feature selection of the blood-biomarkers with 10-fold cross-validated (CV) for lambda selection. Lambda $(\lambda)$, is the regularisation coefficient, and for high values of $\lambda$, LASSO returns a zero vector, whereas when $\lambda$ approaches zero it becomes the standard least squares fit. After correct lambda selection, LASSO minimises the log partial likelihood, shrinking coefficients weights (to zero), decreasing the estimated variance while providing an interpretable final model [2].

Table 2 - Multivariable Cox PH regression of the clinical variables and of clinical variables and bloodbiomarkers included in the derivation dataset. Performance of the model expressed in terms of internal Harrell's c-index, further corrected for optimism by a 10 -fold CV $(*)$ and external one $\left(^{* *}\right)$.

\begin{tabular}{|c|c|c|c|c|c|c|c|c|}
\hline Feature & Hazard Ratio & $\mathrm{p}$-value & $95 \% \mathrm{Cl} \mathrm{HR}$ & c-index & Hazard Ratio & p-value & $95 \% \mathrm{Cl} \mathrm{HR}$ & c-index \\
\hline Gender & & & & 0.66 & & & & 0.70 \\
\hline Male & Reference & & & $0.64 *$ & Reference & & & $0.67^{*}$ \\
\hline Female & 0.50 & $<0.01$ & $0.33-0.75$ & & 0.54 & $<0.01$ & $0.36-0.82$ & \\
\hline WHO-PS & & & & $0.62 * *$ & & & & $0.66^{* *}$ \\
\hline 0 & Reference & & & & Reference & & & \\
\hline 1 & 1.30 & 0.01 & $0.90-1.88$ & & 1.20 & 0.01 & $0.82-1.74$ & \\
\hline$\geq 2$ & 2.65 & & $1.57-4.45$ & & 2.09 & & $1.22-3.58$ & \\
\hline FEV 1s & 1.00 & 0.88 & $0.99-1.01$ & & 1.00 & 0.45 & $0.99-1.00$ & \\
\hline \multicolumn{9}{|l|}{ Lymph nodes } \\
\hline 0 & Reference & & & & Reference & & & \\
\hline 1 & 0.63 & 0.14 & $0.37-1.06$ & & 0.63 & 0.14 & $0.37-1.07$ & \\
\hline 2 & 1.03 & & $0.63-1.66$ & & 1.12 & & $0.69-1.83$ & \\
\hline 3 & 0.59 & & $0.34-1.05$ & & 0.62 & & $0.35-1.09$ & \\
\hline 4 & 0.91 & & $0.57-1.46$ & & 1.10 & & $0.68-1.80$ & \\
\hline $\ln (G T V)\left(\mathrm{cm}^{3}\right)$ & 1.41 & $<0.01$ & $1.21-1.64$ & & 1.28 & $<0.01$ & $1.09-1.50$ & \\
\hline OPN & & & & & 3.31 & 0.01 & $1.31-8.38$ & \\
\hline Cyfra 21-1 & & & & & 1.71 & 0.01 & $1.18-2.50$ & \\
\hline
\end{tabular}

A logarithmic transformation was performed on the blood-biomarker measurements prior to analysis Abbreviations: $\mathrm{Cl}$ - Confidence Interval; HR - Hazard Ratio; WHO-PS - World Health Organization performance status; FEV 1s - forced expiratory volume in 1 second; Lymph nodes refer to the PET positive stations identified; GTV - gross tumour volume (GTV = volume of primary tumour + volume of PET positive lymph node stations); OPN - Osteopontin; Cyfra 21-1 - Cytokeratin fragment 21-1. 
Model performance was evaluated using Harrell's concordance-index (c-index), for which 1 indicates perfect discrimination and a value of 0.5 , no discrimination (no greater than chance expectations). As the model is fit to the data, its performance is usually overestimated. Therefore, the c-index was corrected for this optimism with a 10-fold Cross Validation (CV) [3]. Data were split into 10 subsets. The model coefficients were estimated using $90 \%$ of the data and the model was then tested on the remaining $10 \%$. This procedure was repeated 100 times and the average performance on the test data was computed. The result provides an indication of the model's expected performance on a new dataset. The derived model, apparent (not corrected) and cross-validated performances are shown in Table 2.

\section{External Model validation}

The prognostic index $(\mathrm{PI})$ was computed by multiplying the covariates' values of the validation data with the estimated coefficients: $P I=\sum_{i} B_{i} x_{i}$. Calibration slope was determined on the $\mathrm{Pl}$, by entering the $\mathrm{PI}$ as a variable in the Cox regression and the likelihood ratio test of the equality of this slope to 1 was performed: a slope not significantly different from 1 indicates that the estimated model coefficients can be applied to the new dataset without adaptation, otherwise a recalibration is necessary $[4,5]$.

The calibration slope of the PI was 0.66 and significantly different from $1(p<0.01)$ indicating that the absolute risk prediction was not accurate, being too optimistic. This means that the predictions are too extreme, being too low for low predictions and too high for high estimates (figure in the appendix material). Therefore, the model was recalibrated (Figure 1). 


\section{External validation - calibration curves}

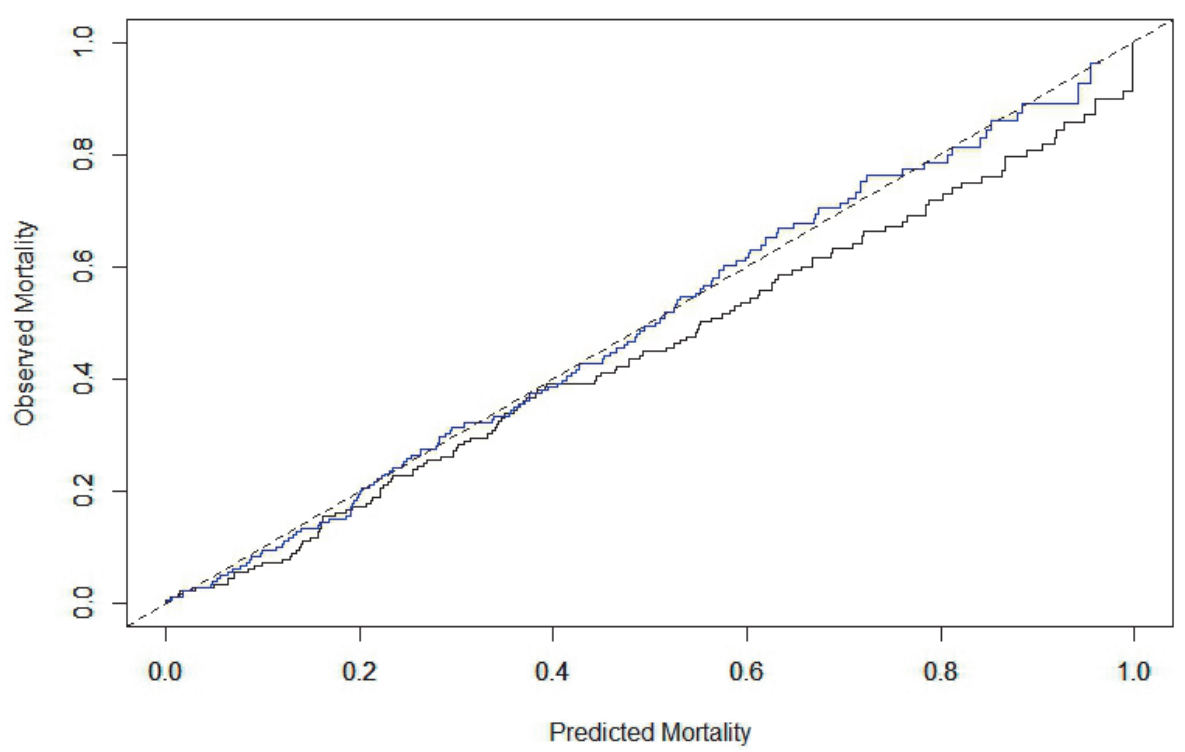

Figure 3 - Calibration curve for the external validation. Slope is statistically below $1(0.66, p<0.01)$, referring to a too optimistic prediction based on the derived model (further away from 45-degree line): under estimations are made for low predictions and high estimations are made for high probabilities. A re-calibration of the model was performed based on the linear predictor slope (overlap with 45-degree line).

\section{Model updating}

The validated model was extended by inputting the PI (after re-calibration) as a fixed variable and further selecting among new proposed blood-biomarkers, following the LASSO approach as for the previous model. The final model can be found in the Table 4 of the main text.

A nomogram was derived, based on the developed model and is presented in the Figure 2 of this appendix. 
Points

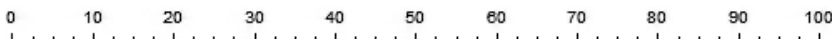

Gender

WHO-PS

FEV 1s

Lymph Nodes

In (GTV)
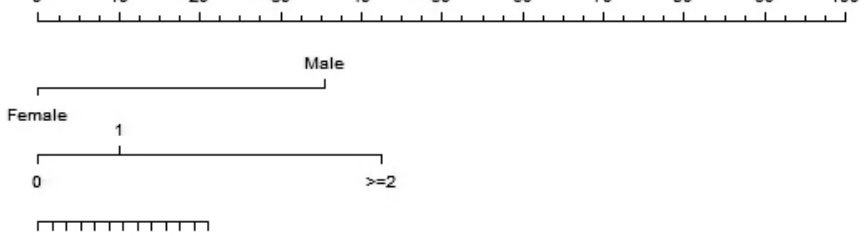

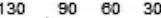

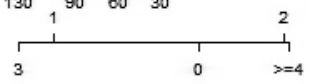

OPN

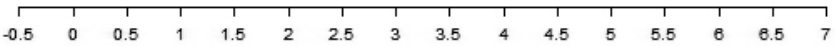

Cyfra 21-1

Total Points
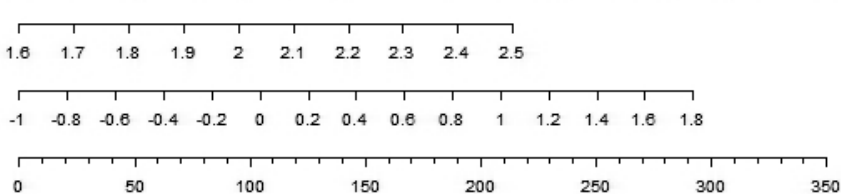

Predicted Value

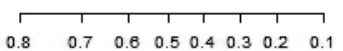

Figure 2 - Nomogram for predicted probability of 2-year survival, using the fitted Cox regression, on the training dataset. To compute the predicted value, gather for each variable the number of points, by drawing a straight upward line from the variable row to the "Points" line on top. Sum the total number of points and locate this value in the "Total points" line. Draw a line straight down from it to the "Predicted Value", as to compute the predicted probability. A logarithmic transformation was performed on the blood-biomarkers measurements prior to analysis. Acronyms: WHO-PS - world health organization performance status; FEV 1s forced expiratory volume in 1 second; Lymph nodes refer to the PET positive stations identified; GTV - gross tumour volume (GTV = volume of primary tumour + volume of PET positive lymph node stations); OPN Osteopontin; Cyfra 21-1 - cytokeratin fragment 21-1.

\section{REFERENCES}

[1] Dehing-Oberije C, Yu S, De Ruysscher D, Meersschout S, Van Beek K, Lievens Y, et al. Development and external validation of prognostic model for 2-year survival of non-small-cell lung cancer patients treated with chemoradiotherapy. Int J Radiat Oncol Biol Phys. 2009 Jun 1;74(2):355-362.

[2] Tibshirani R. The lasso method for variable selection in the Cox model. Stat Med. 199719970429 DCOM19970429;16(4)(0277-6715 (Print)):385-395.

[3] Harrell FE, Jr., Lee KL, Mark DB. Multivariable prognostic models: issues in developing models, evaluating assumptions and adequacy, and measuring and reducing errors. Statistics in medicine. 1996 Feb 28;15(4):361-387.

[4] Royston P, Altman DG. External validation of a Cox prognostic model: principles and methods. BMC medical research methodology. 2013;13:33.

[5] van Houwelingen HC. Validation, calibration, revision and combination of prognostic survival models. Stat Med. 2000 Dec 30;19(24):3401-3415. 


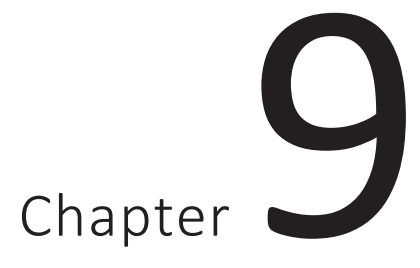

General discussion and concluding remarks

Future perspectives 


\section{GENERAL DISCUSSION AND CONCLUDING REMARKS}

This thesis describes the use of non-invasive sources of information, namely imaging and blood biomarkers for disease management and overall survival assessment for patients with non-small cell lung cancer (NSCLC). Methods for sophisticated image analysis were developed, the so called radiomics, and applied to develop prognostic and predictive models, subsequently validated in external datasets. The importance of blood biomarkers related to hypoxia, inflammation and tumour load for prognostic assessment, was also assessed and later on, extended with newer markers related to immunological processes. Combining the here investigated non-invasive sources of information with clinical parameters, even though not fully explored within this work, is expected to be of great importance in the patient management and improvement of outcomes. Finally, incorporating these in shared decision making tools would be advantageous for both patient and healthcare providers, facilitating existing healthcare routines and patient's awareness on prognostic probabilities.

Final section of this chapter resumes an overview of possible future developments, following the methodologies and conclusions derived along this thesis.

\section{RAPID LEARNING IN RADIOTHERAPY}

Chapter 2 presents an overview on the use of data mining approaches and outcomes modelling as a way to tailor treatment and enhance outcomes in oncology, by means of a rapid-learning health care approach for radiotherapy. Rapid-learning methodologies for health-care in general encompass a continuous learning process from retrospective/prospectively standardly collected data, which evaluation of outcomes, through developed prognostic/predictive tools will iteratively generate new hypothesis for investigation [1]. Additionally, these are aimed to raise awareness at a multilevel clinical care approach, and preventive alertness by engaging patients into screening initiatives for early disease detection. As a result, a more personalized approach is expected, entangled with more participatory patients, but also care providers [2].

Practical evidences of these approaches have been recently demonstrated. A previously devel-oped decision support system (DSS) for NSCLC, was deployed in a typical and busy clinic. This led to the identification of prognostic groups and strengthen its potential for an individualized thera-py, based on the predicted outcomes [3]. This case study illustrates these tools' potential in help-ing clinicians delivering a more reliable prognostic assessment of the disease, thus facilitation the above explained learning phase from multi-institutional data.[4].

Current literature is becoming broader and extensive in reporting the development of prognos-tic/predictive models, not limited to NSCLC, but other solid cancer types [514]. However, few of these make their way into clinical application, which may follow the lack of validation, and there-fore impedes their translation into external and newer data. This is often a reflection of most common problems encountered when sharing 
data across institutes, either due to legal con-straints, patients' own will or even IT infrastructures non-compatibilities.

Radiotherapy-specific data have the potential to be shared across international institutes, without need for burdensome reinventions, assuming its uniformity, completeness and quality is verified. This may follow a standardized protocol, format and semantics, for which controlled vocabulary and ontology are verified, given interoperability between clinical technological infrastructures exists. Nevertheless, patient privacy, particularly across international institutes being a delicate matter should not be disregarded in any way, as application of confidentiality and privacy rules and laws differ between different countries [15].
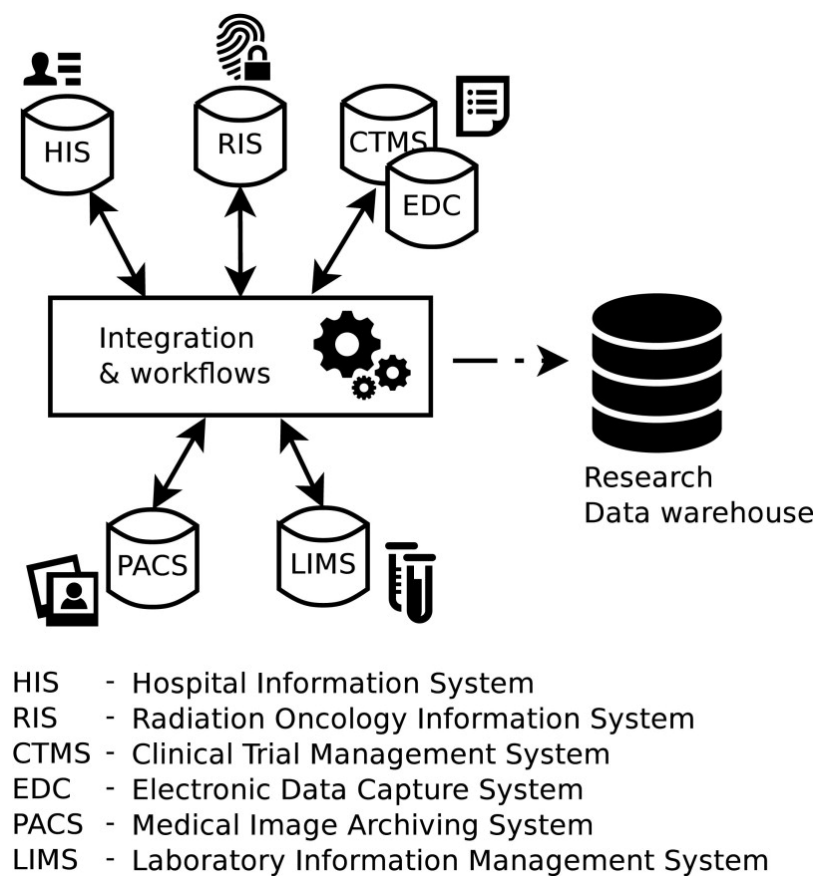

Figure 1 - Rapid-learning systems in radiotherapy require clinical information systems integra-tion. Datasets for research purposes are often stored in date warehouses for further analysis [15].

The current transition from paper records into more sophisticated electronic medical records (EMD), allows for, not only a more structured information, as well as highly increases its sharing potential. Modern data warehouse technologies are expected to provide with high quality data collection and storage. Nevertheless, the initial burdensome in data digitization, is expected to be compensated on the long term, with a higher flexibility of data handling and information extrac-tion for newer studies (Figure 1) [16]. Not to forget that, data collection is the most time-consuming and often prone to errors task, particularly when conducted by humans. 
Following these evidences and current availability of imaging information in dedicated PACS systems (picture archive and communication system - a computer network for digitized radiologic images and reports), opens a branch for data analysis, sharing and validation, that has never found equivalent in the past years.

\section{IMAGING BIOMARKERS FOR NSCLC - RADIOMICS}

Solid tumours are heterogeneous and dissimilar in shape and size amongst patients. The possibility to describe this heterogeneity in a non-invasive manner, derive prognostic information from it, and potentially capture its underlying genomic and proteomic profile is the main hypothesis behind Radiomics, presented in Chapter 3. In this chapter, even though not introducing a novel concept, it definitely brings into light a more sophisticated workflow of image analysis, from high quality imaging data acquisition to the high-throughput quantification of imaging features derived from a robustly defined region-of-interest (ROI). The large spectrum of imaging features retrieved from this ROI is then to be analyzed through refined statistical methods and modelling techniques with the goal of inferring useful, predictive, and prognostic information. Following this conceptual presentation of the project, many studies were conducted and are discussed in the following chapters.

This thesis particularly deals with the metabolic distribution of ${ }^{18} \mathrm{~F}$-fluorodeoxyglucose (FDG), as measured by positron emission tomography (PET) for NSCLC patients, treated radically with radiotherapy. Radiomics features analyzed include descriptors of shape and size $(n=9)$, first order statistics $(n=16)$, texture information (second order statistics) - with the three sub-categories run length grey level - RLGL $(n=11)$, grey level co-occurrence $-\operatorname{GLCM}(n=22)$, and grey level size zone matrices $\operatorname{GLSZM~(n=11),~and~intensity-volume~histogram~features~(IVH)~(~} n=45)$ (Figure 2). A mathematical explanation of these features is given in the appendix to Chapter 5, with exception of IVH metrics, further introduced in Chapter 4.

IVH features are similar in concept to the dose-volume histograms (DVH) deeply used in the radiotherapy field, but instead of dose distributions, describe tumour's FDG uptake distribution as a function of its volume, and vice-versa [17]. These include descriptors of absolute and relative volume for increasing relative intensities of 10 up to $90 \%$ in steps of $10 \%$ (AVRIx and RVRIx); descriptors of absolute and mean intensities enclosed with increasingly relative volume portions from 10 up to $90 \%$ in steps of $10 \%$ (AIRVx and MIRVx); metabolic tumour volume (MTV) and total lesion glycolysis (TLG) defined for increasing relative intensities of 10 up to $90 \%$ in steps of $10 \%$ (MTVRIx and TLGRIX). Note that MIRV, MTV and TLG curves were introduced later in the in-house develop toolbox for feature extraction, and therefore not accounted for the stability analysis performed in Chapter 5. A similar analysis was later conducted before their inclusion in the studies presented in chapters 6 and 7. 
Radiomics approach is the precursor of the next four chapters that in an initial stage started by investigating whether IVH, a particular sub-category of first order statistics metrics, would provide extra information, in comparison to the commonly analyzed features as derived from the FDG-PET signal: maximum, peak and mean standard uptake value (SUV). Chapter 4 describes the results of analyzing the FDG-PET signal at the baseline scan (prior to radiotherapy delivery) in a large dataset of 220 NSCLC patients treated radically at our institute. Tumour heterogeneous FDG uptake is intrinsically dependent on its microenvironment, that can be more accurately describe with IVH metrics, providing a more complete overview of the complex tumour intensity distribution across total tumour volume, instead of narrowed to a single high uptake value, as maximum or peak SUV $[18,19]$. All IVH metrics were evaluated in this chapter, with the exception of TLGRIx and MIRVx, only investigated in Chapters 6 and 7, as they were later included in the imaging analysis toolbox. Results summarized in this chapter report on important findings of relative volume enclosing the higher relative intensity being a significant prognostic factor for overall survival, which could not be concluded for the maximum or peak SUV. Even though lacking external validation, these positive results were introductory for the extended imaging analysis in which more radiomics features were included.

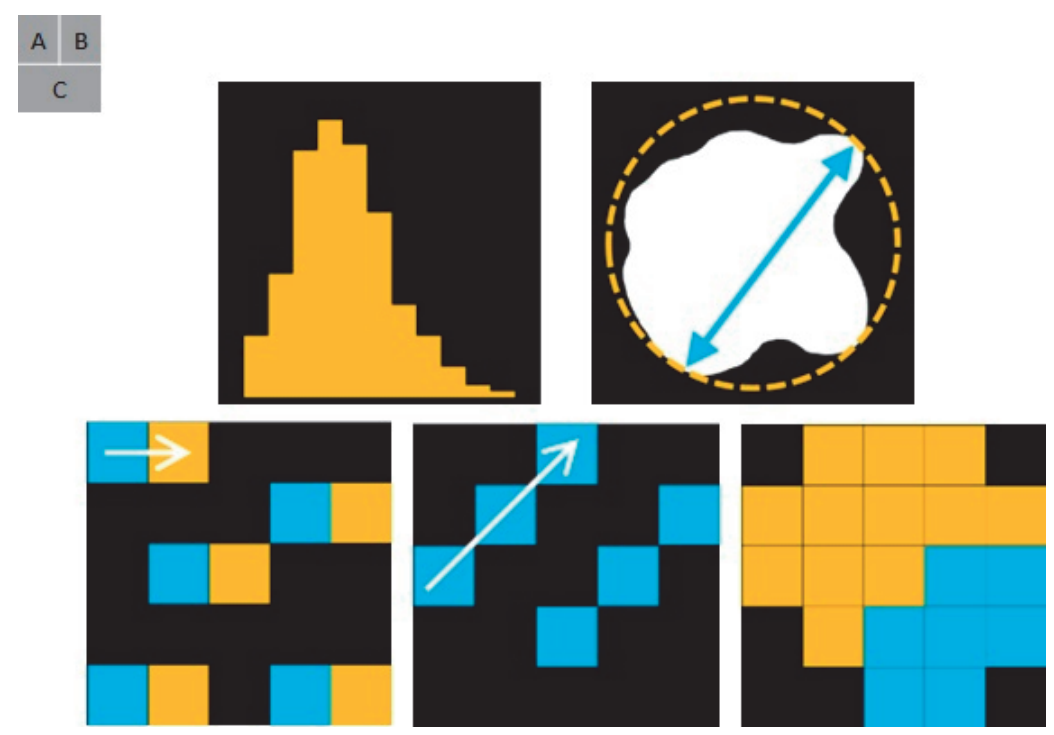

Figure 2 - Radiomics features comprised descriptors of (A) first order statistics, (B) shape and size and (C) texture information, describing spatial distribution of voxel intensities and deter-mined by considering 26 connected voxels (i.e. voxels were considered to be neighbors in all 13 directions in three dimensions) at a distance of 1 voxel. Features derived from GLCM and RLGL were calculated by averaging their value over all 13 directions. 
Chapter 5 presents an analysis on the stability of Radiomics features, assessed in a test-retest scenario and through multiple radiation oncologist's primary tumour delineations. Test-retest included the analysis of PET scans from 11 liver cancer patients, acquired one day apart with no treatment administered in between. Multiple observers analysis involved the investigation of Radiomics features' robustness against manual and independent delineations. These were per-formed by five independent and experienced radiation oncologist, who were required to segment the primary tumour on pre-radiotherapy fused PET-CT scans, blinded to each other's delineations. Solely 77 out of 118 features achieved a high stability in both tests, defined as an intraclass correlation (ICC) larger than 0.85. These were included in the subsequent analyses in the studies pre-sented in Chapters 6 and 7.

A bottleneck of imaging analysis, and particularly PET, is its dependence on the followed protocol for FDG injection, scan acquisition and reconstruction as well correction protocols. In this scenar-io, stability tests become an important and crucial step in the imaging analysis and prognostic modelling, particularly when dealing with independent and external validation scans, often per-formed in different scanners. Previous studies have addressed this matter, particularly for CT imaging [20-22]. One should however keep in mind that this step provides a more reliable ap-proach when performed in scans acquired with the same scanner and following the same acquisi-tion protocols, as data in the development datasets for sequent model derivation. This should be deeply considered in the future, by means of using phantom acquisitions, for instance. However, and even though with this limitation, one can rely on this approach to provide a good estimation for accurate selection amongst the whole spectrum of Radiomics features in order to proceed analysis with the most stable and robust ones.

In Chapter 6 the changes of radiomics features early during radiotherapy were analyzed, the so-called "Delta Radiomics" approach. Few studies have already addressed the importance of meas-uring the changes in FDG uptake patterns, early during treatment, in order to improve outcomes in a stage at which treatment adaptation is still a possibility. These have included mainly first order descriptors, metabolic tumour volume - MTV (the highly uptake region above a defined threshold) and total lesion glycolysis - TLG (defined as the product of MTV with its enclosed mean SUV) [23-27]. Here, a multivariable model was built, based on early changes in Radiomics features in 52 NSCLC patients, and subsequently validated in two independent datasets ( $n=32$ and 26). The final model included, at least, one variable from each category. To note that the univariable percentage variation of most commonly assessed features (maximum, mean and peak SUV, and tumour volume) was not statistically significant. In addition to the benefit of combining heterogeneous imaging descriptors, as offered with the Radiomics approach, the greatest advantage here documented refers to the validation performed. The use of two independent and external datasets offers protection against model fit in independent data of the one used in the development phase. This is a crucial step when moving towards the clinical 
application, offering a better insight on how the derived models would fit in new data. A satisfactory internal performance of $0.66(p<0.01)$ was achieve, here measured as the concordance-index (ranging from 0.5 - not better than the expected chance, up to 1 perfect fit). Independent validation presented performances of $0.64(p<0.01)$ and 0.57 $(p=0.27)$. The lower external performance, particularly for the second validation dataset and further discussed in the corresponding chapter, reinforces the debate on a uniform acquisition protocol, not only of FDG injection and reconstruction parameters, but also timing. The repeated acquisition within treatment, when following an homogenous protocols amongst institutes will provide a higher accuracy assessment of disease evolution. It is, therefore desired a combination of efforts between institutes as to derive homogeneous imaging acquisition protocols, in order to gather a large enough number of observations that would allow for a more precise and validated predic-tive model.

Finally, in Chapter 7 a Radiomics approach is conducted to both primary tumour and the metastat-ic lymph nodes of NSCLC. Main hypothesis of this chapter was that metastatic lymph nodes, and its associated higher metastatic potential, do provide extra prognostic value in addition to the primary tumour, and for this purpose, a whole set of stable radiomics features were extracted and analyzed for both structures. These were used to fit a prognostic model on the derivation dataset ( $n=262$ patients) and further validated on an external one $(n=50)$. Main conclusion of this study was that a model selecting amongst features exclusively extracted from the metastatic lymph nodes achieved a statistically higher performance than similar modeling approach based solely on the primary tumour. External validation of these models showed however that the best fit was achieved when combining both sources of information, meaning features from metastatic lymph nodes and primary tumour, which achieved a performance of 0.62 - external 0.59. Other ap-proaches verified performances of 0.53 - external 0.54 , when derived from tumour, and 0.62 - external 0.56 from metastatic lymph nodes. All performances are expressed in terms of concord-ance-index.

The analysis of imaging traits derived from metastatic lymph nodes, even though expected to provide with relevant insight of disease prognosis, is still very limited in the current literature $[28,29]$, with more relevance currently still being given to the primary tumour alone [30-32]. This study, being a newer approach in the imaging analysis for NSCLC, and despite its limitations of not providing a stratified analysis per metastatic node station affected (and further, discussed in the corresponding chapter), clearly brings into light the extra source of information that these structures provide for a more accurate prognostic assessment. This gains even more attention, particularly when it also confirmed the higher prognostic value associated with the number of metastatic lymph nodes, when compared to the location-based pathological nodal stage classifi-cation [33].

To conclude this section, one of its limitations refers to the only outcome measured and analyzed: overall survival. This drawback is expected to be addressed in the future, 
specifically when corre-lation between imaging traits accessed prior to radiotherapy delivery, have been reportedly related to progression free survival, tumour control and relapse $[23,34,35]$. In order to validate these findings, prospective data collection should be improved to facilitate the incorporation of mentioned outcomes in future analyses, not possible to perform at the time that this work was conducted. Literature has also reported the investigation of response assessment and prognosis for NSCLC patients following other therapeutic approaches, not limited to radiotherapy as presented in this thesis, namely erlotinib therapy and chemotherapy alone [36-39]. However, and despite the positive findings, this has been restricted to the analysis of higher regions of uptake, for which a full Radiomics approach would be of expected advantage, but, still not proved.

\section{BLOOD BIOMARKERS FOR NSCLC}

In this thesis the seek for non-invasive ways to accurately describe tumour and further characterize its prognostic profiles followed two approaches, imaging and blood respectively. In Chapter 8 a study on blood-biomarkers related to hypoxia, inflammation and tumour load, was performed and validated in an independent cohort, comprising a total of over 350 patients.

The use of blood-biomarkers has been receiving much attention in the past years as for lung cancer, tissue collection by means of biopsy is a difficult process as already mentioned. In this scenario, blood collection by veniculture offers an attractive alternative. Blood-biomarkers selec-tion was based on proven evidence of their prognostic value and association with treatment outcome (survival or tumour control), restricted to studies with a minimum of 100 patients and narrowed to markers that could be measured in daily clinical practice (to ensure its applicability in clinical practice). The selection comprised markers related to hypoxia [osteopontin (OPN) and carbonic anhydrase IX (CA-IX)], inflammation [interleukin 6 (IL-6), IL-8, and C-reactive protein (CRP)], and tumour load [carcinoembryonic antigen (CEA) and cytokeratin fragment (CYFRA 21-1)] [40-54].

Even though many studies have already addressed the utility of different bloodbiomarkers indi-vidually, we here that analysis to a multivariable approach including dissimilar types of markers in a large set of NSCLC patients $(n=182)$, further validated in an independent and equally large dataset $(n=181)$. The obtained results reported on a positive improvement of a validated clinical model including World Health Organization performance-status, forced expiratory volume in 1 second, number of positive lymph node stations, and gross tumour volume, with the addition of a marker of hypoxia (OPN) and one of tumour load (Cyfra 21-1) [7]. This model was then extended by selecting among newer markers, only available in the development dataset, including immunogenic markers: alpha-2-macroglobulin ( $\alpha 2 \mathrm{M})$, serum IL-2 receptor (sIL2R), tolllike receptor 4 (TLR4), and vascular endothelial growth factor (VEGF) [43, 55-62]. The 
selection of these markers followed a similar rationale as previously described, further testing the possibility to identify surrogates for immunological response, which could therefore be incorporated into immunotherapy assessment studies. This was in response to evidence that immunotherapy has positive effects on NSCLC [63-67]. Still, this was an exploratory step, which still requires further validation.

In summary, we obtained positive results for the use of blood-biomarkers to predict overall sur-vival. Similarly to the closing remarks of previous section, other outcomes would also be of inter-est. Recent literature reported on a panel of 6 serum biomarkers (including Cyfra 21-1), related to overall survival assessment, which however could not characterized the disease in terms of its development and spread of brain metastasis [68]. As performed for imaging biomarkers in Chap-ter 6, the early response assessment for disease progression and treatment effectiveness by means of blood collection during treatment, still remain and unanswered hypothesis. To close this section discussion, some of the here investigated markers, particularly CEA and CYFRA 21-1, have been reported as a valuable diagnostic tool, providing an early and high sensitive identification of newer lung neoplasms, reinforcing its high potential for disease management [69]. This list of potential biomarkers can also be expanded with DNArelated ones, retrieved from saliva samples, but here not explored [70].

\section{FUTURE PERSPECTIVES}

The imaging analysis, here restricted to FDG-PET, has always the potential to be extended to, or complemented with other imaging modalities, as CT, magnetic resonance imaging (MRI), single-photon emission computerized tomography (SPECT), to name a few. For its clinical application, a reliable and reproducible assessment of radiomics features is required, as demonstrated in chap-ter 5. Therefore, a clear definition of imaging protocols, testing and development of phantoms should be in the agenda for the next developments. This should be conducted in a longitudinal manner, including not only the development datasets acquisitions, but also the ones used for validation purposes.

Still in the field PET signal analysis, interesting to address would be the extension of tumour me-tabolism assessment, here measured by FDG uptake, with different tracers, namely for hypoxia and diffusion. Hypoxia is known to be a prevalent effect on NSCLC, for which a previous study already reported on the combined advantage of analyzing PET with an appropriate hypoxia mark-er and MRI for early response assessment during treatment $[71,72]$. Also diffusion markers, have been gaining more attention nowadays, with reported evidences that early variation during treatment of $18 \mathrm{~F}$-fluorothymidine $(F L T)$ is a surrogate for treatment response in NSCLC patients treated with concurrent (chemo)radiotherapy [73]. Aligned with this, and in order to reduce patient's burden with potentially unnecessary radioactive exposure, proper way of identifying which 
marker to use based on Radiomics analysis of anatomic information (for instance, CT), would greatly increase imaging modalities usage. Reduction of costs would also be an economic benefit, as a more tailored scanning protocol would be performed, instead of a one-size-fits-all approach.

Common analysis of the imaging information nowadays is circumscribed to the primary tumour for many of the solid cancer diseases. We presented in chapter 7 an analysis for which a great value appears to be laying on the metastatic lymph nodes for NSCLC. Future work planning should include the analysis of early variation of these metrics derived from the metastatic nodes, follow-ing preliminary evidences that commonly assessed first order statistic metrics do provide with predictive information, when assessed early after completion of first cycle of treatment [28]. This approach should thoroughly be considered to be extended to other disease sites, in particular head and neck cancer, for which nodes involvement is often a worse prognostic factor [74]. Next step would definitely be the extension of these methodologies to other structures, in particularly distant metastasis, or even complete organs, for instance total lung.

The combination of the different sources of information (imaging and blood biomarkers) should also thoroughly be considered in a future investigation. A recent study showed the added value in combining plasma VEGF-C and PET signal to predict nodal disease of NSCLC patients [75]. Within the timeframe dedicated to this research, it was however, not possible to complement the here presented findings with a combined study of both sources of information.

Preliminary results suggested an association between Radiomics features of a prognostic signa-ture derived on a large dataset of NSCLC and gene expression, using gene-set enrichment analysis (GSEA) [76]. These observations were based on preradiotherapy CT images of a sample of 89 NSCLC patients. Linking Radiomics features and blood biomarkers to genotype, would definitely overcome the current main drawback on NSCLC management, referring to tissue sampling. Thus, offering a great gain in treatment response assessment and prediction of outcomes.

Finally, this thesis focused on development and validation of models, incorporating imaging and blood biomarkers. Following the rationale already discussed, one should keep in mind that there are always concerns with generalization, particularly with model development. Frequently, this appears to be associated with the limited number of observations for validation. Nonetheless, development datasets are themselves often not large or heterogeneous enough, implying that derived models lack enough "knowledge" to make accurate predictions on new data. This bridges back to the need of a more data-sharing mindset, data accrual and re-use, following the iterative learning process of rapid-learning approaches. The translation of these models into clinical practice should include the development of tools capable of providing with individualized prognostic information, and suitable for both clinicians and patients. Moreover, these should consider clini-cians and patients expectations and preferences. 
As a general remark and to close this future perspectives section, many of the approaches here presented are highly desirable to be applied to other solid cancer sites, as head and neck and colorectal cancer, for instance. In the possession of accurately defined and validated prognostic and predictive models, one can expect to move towards a more precise and individualized radio-therapy approach, instead of a standard treatment. This thesis provides innovative factors that can be applied in the near future for selection of patients for randomized clinical trials or a more individualized treatment decision making. It paves the way for an automated imaging analysis pipeline that can be used in daily clinical practice and which will ultimately optimize treatment decisions and improve outcome for cancer patients. 


\section{REFERENCES}

[1] Abernethy AP, Etheredge LM, Ganz PA, Wallace P, German RR, Neti C, et al. Rapid-learning system for cancer care. Journal of clinical oncology : official journal of the American Society of Clinical Oncology. 2010 Sep 20;28(27):4268-4274.

[2] Shaikh AR, Butte AJ, Schully SD, Dalton WS, Khoury MJ, Hesse BW. Collaborative biomedicine in the age of big data: the case of cancer. Journal of medical Internet research. 2014;16(4):e101.

[3] Dekker A, Vinod S, Holloway L, Oberije C, George A, Goozee G, et al. Rapid learning in practice: a lung cancer survival decision support system in routine patient care data. Radiotherapy and oncology : journal of the European Society for Therapeutic Radiology and Oncology. 2014 Oct;113(1):47-53.

[4] Lambin P, Zindler J, Vanneste B, Van De Voorde L, Eekers D, Compter I, et al. Decision Support Systems for Personalized and Participative Radiation Oncology. Advanced drug delivery reviews. 2016 Jan 13.

[5] Wishart GC, Azzato EM, Greenberg DC, Rashbass J, Kearins O, Lawrence G, et al. PREDICT: a new UK prognostic model that predicts survival following surgery for invasive breast cancer. Breast cancer research : BCR. 2010;12(1):R1.

[6] Dehing-Oberije C, De Ruysscher D, van der Weide H, Hochstenbag M, Bootsma G, Geraedts W, et al. Tumour volume combined with number of positive lymph node stations is a more important prognostic factor than TNM stage for survival of non-small-cell lung cancer patients treated with (chemo) radiotherapy. International journal of radiation oncology, biology, physics. 2008 Mar 15;70(4):10391044.

[7] Dehing-Oberije C, Yu S, De Ruysscher D, Meersschout S, Van Beek K, Lievens Y, et al. Development and external validation of prognostic model for 2-year survival of non-small-cell lung cancer patients treated with chemoradiotherapy. International journal of radiation oncology, biology, physics. 2009 Jun $1 ; 74(2): 355-362$.

[8] Oberije C, De Ruysscher D, Houben R, van de Heuvel M, Uyterlinde W, Deasy JO, et al. A Validated Prediction Model for Overall Survival From Stage III Non-Small Cell Lung Cancer: Toward Survival Prediction for Individual Patients. International journal of radiation oncology, biology, physics. 2015 Jul 15;92(4):935-944.

[9] Marks LB, Bentzen SM, Deasy JO, Kong FM, Bradley JD, Vogelius IS, et al. Radiation dose-volume effects in the lung. International journal of radiation oncology, biology, physics. 2010 Mar 1;76(3 Suppl):S70-76.

[10] van Stiphout RG, Lammering G, Buijsen J, Janssen MH, Gambacorta MA, Slagmolen P, et al. Development and external validation of a predictive model for pathological complete response of rectal cancer patients including sequential PET-CT imaging. Radiotherapy and oncology : journal of the European Society for Therapeutic Radiology and Oncology. 2011 Jan;98(1):126-133.

[11] Valentini V, van Stiphout RG, Lammering G, Gambacorta MA, Barba MC, Bebenek M, et al. Nomograms for predicting local recurrence, distant metastases, and overall survival for patients with locally advanced rectal cancer on the basis of European randomized clinical trials. Journal of clinical oncology : official journal of the American Society of Clinical Oncology. 2011 Aug 10;29(23):3163-3172.

[12] Egelmeer AG, Velazquez ER, de Jong JM, Oberije C, Geussens Y, Nuyts S, et al. Development and validation of a nomogram for prediction of survival and local control in laryngeal carcinoma patients treated with radiotherapy alone: a cohort study based on 994 patients. Radiotherapy and oncology : journal of the European Society for Therapeutic Radiology and Oncology. 2011 Jul;100(1):108-115.

[13] Creutzberg CL, van Stiphout RG, Nout RA, Lutgens LC, Jurgenliemk-Schulz IM, Jobsen JJ, et al. Nomograms for prediction of outcome with or without adjuvant radiation therapy for patients with endometrial cancer: a pooled analysis of PORTEC-1 and PORTEC-2 trials. International journal of radiation oncology, biology, physics. 2015 Mar 1;91(3):530-539.

[14] Rios Velazquez E, Hoebers F, Aerts HJ, Rietbergen MM, Brakenhoff RH, Leemans RC, et al. Externally validated HPV-based prognostic nomogram for oropharyngeal carcinoma patients yields more accurate predictions than TNM staging. Radiotherapy and oncology : journal of the European Society for Therapeutic Radiology and Oncology. 2014 Dec;113(3):324-330. 
[15] Skripcak T, Belka C, Bosch W, Brink C, Brunner T, Budach V, et al. Creating a data exchange strategy for radiotherapy research: towards federated databases and anonymised public datasets. Radiotherapy and oncology : journal of the European Society for Therapeutic Radiology and Oncology. 2014 Dec;113(3):303-309.

[16] Roelofs E, Persoon L, Nijsten S, Wiessler W, Dekker A, Lambin P. Benefits of a clinical data warehouse with data mining tools to collect data for a radiotherapy trial. Radiotherapy and oncology : journal of the European Society for Therapeutic Radiology and Oncology. 2013 Jul;108(1):174-179.

[17] JC LA, Herrero MA, Nunez L. A dose-volume histogram based decision-support system for dosimetric comparison of radiotherapy treatment plans. Radiation oncology (London, England). 2015;10(1):263.

[18] El Naqa I, Grigsby P, Apte A, Kidd E, Donnelly E, Khullar D, et al. Exploring feature-based approaches in PET images for predicting cancer treatment outcomes. Pattern recognition. 2009 Jun 1;42(6):1162-1171.

[19] van Velden FH, Cheebsumon P, Yaqub M, Smit EF, Hoekstra OS, Lammertsma AA, et al. Evaluation of a cumulative SUV-volume histogram method for parameterizing heterogeneous intratumoural FDG uptake in non-small cell lung cancer PET studies. European journal of nuclear medicine and molecular imaging. 2011 Sep;38(9):1636-1647.

[20] Balagurunathan Y, Gu Y, Wang H, Kumar V, Grove O, Hawkins S, et al. Reproducibility and Prognosis of Quantitative Features Extracted from CT Images. Translational oncology. 2014 Feb;7(1):72-87.

[21] Hunter LA, Krafft S, Stingo F, Choi H, Martel MK, Kry SF, et al. High quality machine-robust image features: identification in nonsmall cell lung cancer computed tomography images. Medical physics. 2013 Dec;40(12):121916.

[22] Mackin D, Fave X, Zhang L, Fried D, Yang J, Taylor B, et al. Measuring Computed Tomography Scanner Variability of Radiomics Features. Investigative radiology. 2015 Nov;50(11):757-765.

[23] Yossi S, Krhili S, Muratet JP, Septans AL, Campion L, Denis F. Early assessment of metabolic response by 18F-FDG PET during concomitant radiochemotherapy of non-small cell lung carcinoma is associated with survival: a retrospective single-center study. Clinical nuclear medicine. 2015 Apr;40(4):e215-221.

[24] Kim S, Oh SW, Kim JS, Kim KH, Kim YK. Prediction of response by FDG PET early during concurrent chemoradiotherapy for locally advanced non-small cell lung cancer. Radiation oncology journal. 2014 Dec;32(4):231-237.

[25] Toma-Dasu I, Uhrdin J, Lazzeroni M, Carvalho S, van Elmpt W, Lambin P, et al. Evaluating tumour response of non-small cell lung cancer patients with (1)(8)F-fludeoxyglucose positron emission tomography: potential for treatment individualization. International journal of radiation oncology, biology, physics. 2015 Feb 1;91(2):376-384.

[26] Fledelius J, Khalil AA, Hjorthaug K, Frokiaer J. Using positron emission tomography (PET) response criteria in solid tumours (PERCIST) 1.0 for evaluation of 2'-deoxy-2'-[18F] fluoro-D-glucose-PET/CT scans to predict survival early during treatment of locally advanced non-small cell lung cancer (NSCLC). Journal of medical imaging and radiation oncology. 2015 Dec 18.

[27] van Elmpt W, Ollers M, Dingemans AM, Lambin P, De Ruysscher D. Response assessment using 18F-FDG PET early in the course of radiotherapy correlates with survival in advanced-stage non-small cell lung cancer. Journal of nuclear medicine : official publication, Society of Nuclear Medicine. 2012 Oct;53(10):1514-1520.

[28] Markovina S, Duan F, Snyder BS, Siegel BA, Machtay M, Bradley JD. Regional Lymph Node Uptake of [(18)F]Fluorodeoxyglucose After Definitive Chemoradiation Therapy Predicts Local-Regional Failure of Locally Advanced Non-Small Cell Lung Cancer: Results of ACRIN 6668/RTOG 0235. International journal of radiation oncology, biology, physics. 2015 Nov 1;93(3):597-605.

[29] Li H, Becker N, Raman S, Chan TC, Bissonnette JP. The value of nodal information in predicting lung cancer relapse using 4DPET/4DCT. Medical physics. 2015 Aug;42(8):4727-4733.

[30] Ohri N, Duan F, Machtay M, Gorelick JJ, Snyder BS, Alavi A, et al. Pretreatment FDG-PET metrics in stage III non-small cell lung cancer: ACRIN 6668/RTOG 0235. Journal of the National Cancer Institute. 2015 Apr;107(4). 
[31] Usmanij EA, de Geus-Oei LF, Bussink J, Oyen WJ. Update on F-18-fluoro-deoxy-glucose-PET/computed tomography in nonsmall cell lung cancer. Current opinion in pulmonary medicine. 2015 Jul;21(4):314321.

[32] Paesmans M, Garcia C, Wong CY, Patz EF, Jr., Komaki R, Eschmann S, et al. Primary tumour standardised uptake value is prognostic in nonsmall cell lung cancer: a multivariate pooled analysis of individual data. The European respiratory journal. 2015 Dec;46(6):1751-1761.

[33] Wei S, Asamura H, Kawachi R, Sakurai H, Watanabe S. Which is the better prognostic factor for resected non-small cell lung cancer: the number of metastatic lymph nodes or the currently used nodal stage classification? Journal of thoracic oncology : official publication of the International Association for the Study of Lung Cancer. 2011 Feb;6(2):310-318.

[34] Jeong JU, Chung WK, Nam TK, Song JY, Yoon MS, Kim YC, et al. Early metabolic response on 18Ffluorodeoxyglucose-positron-emission tomography/computed tomography after concurrent chemoradiotherapy for advanced stage III non-small cell lung cancer is correlated with local tumour control and survival. Anticancer research. 2014 May;34(5):2517-2523.

[35] Sura S, Greco C, Gelblum D, Yorke ED, Jackson A, Rosenzweig KE. (18)F-fluorodeoxyglucose positron emission tomography-based assessment of local failure patterns in non-small-cell lung cancer treated with definitive radiotherapy. International journal of radiation oncology, biology, physics. 2008 Apr 1;70(5):1397-1402.

[36] Hachemi M, Couturier O, Vervueren L, Fosse P, Lacoeuille F, Urban T, et al. [(1)(8)F]FDG positron emission tomography within two weeks of starting erlotinib therapy can predict response in non-small cell lung cancer patients. PloS one. 2014;9(2):e87629.

[37] Ordu C, Selcuk NA, Erdogan E, Angin G, Gural Z, Memis H, et al. Does early PET/CT assesment of response to chemotherapy predicts survival in patients with advanced stage non-small-cell lung cancer? Medicine. 2014 Dec;93(28):e299.

[38] Cook GJ, O'Brien ME, Siddique M, Chicklore S, Loi HY, Sharma B, et al. Non-Small Cell Lung Cancer Treated with Erlotinib: Heterogeneity of (18)F-FDG Uptake at PET-Association with Treatment Response and Prognosis. Radiology. 2015 Sep;276(3):883-893.

[39] Han EJ, Yang YJ, Park JC, Park SY, Choi WH, Kim SH. Prognostic value of early response assessment using 18F-FDG PET/CT in chemotherapy-treated patients with non-small-cell lung cancer. Nuclear medicine communications. 2015 Dec;36(12):1187-1194.

[40] Donati V, Boldrini L, Dell'Omodarme M, Prati MC, Faviana P, Camacci T, et al. Osteopontin expression and prognostic significance in non-small cell lung cancer. Clinical cancer research : an official journal of the American Association for Cancer Research. 2005 Sep 15;11(18):6459-6465.

[41] Fiala O, Pesek M, Finek J, Topolcan O, Racek J, Minarik M, et al. High serum level of C-reactive protein is associated with worse outcome of patients with advanced-stage NSCLC treated with erlotinib. Tumour Biol. 2015 Jun 19.

[42] Mendes F, Antunes C, Abrantes AM, Goncalves AC, Nobre-Gois I, Sarmento AB, et al. Lung cancer: the immune system and radiation. Br J Biomed Sci. 2015;72(2):78-84.

[43] Ostheimer C, Bache M, Guttler A, Kotzsch M, Vordermark D. A pilot study on potential plasma hypoxia markers in the radiotherapy of non-small cell lung cancer. Osteopontin, carbonic anhydrase IX and vascular endothelial growth factor. Strahlentherapie und Onkologie : Organ der Deutschen Rontgengesellschaft [et al]. 2014 Mar;190(3):276-282

[44] Rud AK, Boye K, Oijordsbakken M, Lund-Iversen M, Halvorsen AR, Solberg SK, et al. Osteopontin is a prognostic biomarker in non-small cell lung cancer. BMC Cancer. 2013;13:540.

[45] Sanmamed MF, Carranza-Rua O, Alfaro C, Onate C, Martin-Algarra S, Perez G, et al. Serum interleukin-8 reflects tumour burden and treatment response across malignancies of multiple tissue origins. Clin Cancer Res. 2014 Nov 15;20(22):5697-5707.

[46] Wang J, Yi Y, Li B, Wang Z, Sun H, Zhang P, et al. CYFRA21-1 can predict the sensitivity to chemoradiotherapy of non-small-cell lung carcinoma. Biomarkers. $2010 \mathrm{Nov}$;15(7):594-601.

[47] Wang J, Zhang N, Li B, Wang Z, Sun H, Yi Y, et al. Decline of serum CYFRA21-1 during chemoradiotherapy of NSCLC: a probable predictive factor for tumour response. Tumour Biol. 2011 Aug;32(4):689-695. 
[48] Zou XL, Wang C, Liu KE, Nie W, Ding ZY. Prognostic significance of osteopontin expression in non-smallcell lung cancer: A meta-analysis. Mol Clin Oncol. 2015 May;3(3):633-638.

[49] Liu Y, Gu X, Lin Q, Tian T, Shao L, Yuan C, et al. Prognostic significance of osteopontin in patients with non-small cell lung cancer: results from a meta-analysis. International journal of clinical and experimental medicine. 2015;8(8):12765-12773.

[50] Jing X, Huang C, Zhou H, Li C, Fan L, Chen J, et al. Association between serum C-reactive protein value and prognosis of patients with non-small cell lung cancer: a meta-analysis. International journal of clinical and experimental medicine. 2015;8(7):10633-10639.

[51] Ilie M, Mazure NM, Hofman V, Ammadi RE, Ortholan C, Bonnetaud C, et al. High levels of carbonic anhydrase IX in tumour tissue and plasma are biomarkers of poor prognostic in patients with non-small cell lung cancer. British journal of cancer. 2010 May 25;102(11):1627-1635.

[52] Gomes M, Coelho A, Araujo A, Azevedo A, Teixeira AL, Catarino R, et al. IL-6 polymorphism in non-small cell lung cancer: a prognostic value? Tumour Biol. 2015 May;36(5):3679-3684.

[53] Liao C, Yu Z, Guo W, Liu Q, Wu Y, Li Y, et al. Prognostic value of circulating inflammatory factors in nonsmall cell lung cancer: a systematic review and meta-analysis. Cancer biomarkers : section A of Disease markers. 2014;14(6):469-481.

[54] Zhang ZH, Han YW, Liang H, Wang LM. Prognostic value of serum CYFRA21-1 and CEA for non-small-cell lung cancer. Cancer medicine. 2015 Nov;4(11):1633-1638.

[55] Bien E, Balcerska A. Serum soluble interleukin 2 receptor alpha in human cancer of adults and children: a review. Biomarkers : biochemical indicators of exposure, response, and susceptibility to chemicals. 2008 Feb;13(1):1-26.

[56] De Vita F, Turitto G, di Grazia M, Frattolillo A, Catalano G. Analysis of interleukin-2/interleukin-2 receptor system in advanced non-small-cell lung cancer. Tumouri. 1998 Jan-Feb;84(1):33-38.

[57] Fu HY, Li C, Yang W, Gai XD, Jia T, Lei YM, et al. FOXP3 and TLR4 protein expression are correlated in nonsmall cell lung cancer: implications for tumour progression and escape. Acta histochemica. 2013 Mar;115(2):151-157.

[58] Kawashima O, Kamiyoshihara M, Sakata S, Endo K, Saito R, Morishita Y. The clinicopathological significance of preoperative serum-soluble interleukin-2 receptor concentrations in operable non-smallcell lung cancer patients. Annals of surgical oncology. 2000 Apr;7(3):239-245.

[59] Naumnik W, Chyczewska E. The clinical significance of serum soluble interleukin 2 receptor (sIL-2R) concentration in lung cancer. Folia histochemica et cytobiologica / Polish Academy of Sciences, Polish Histochemical and Cytochemical Society. 2001;39 Suppl 2:185-186.

[60] Oh JH, Craft JM, Townsend R, Deasy JO, Bradley JD, El Naqa I. A bioinformatics approach for biomarker identification in radiation-induced lung inflammation from limited proteomics data. Journal of proteome research. 2011 Mar 4;10(3):1406-1415.

[61] Rades D, Setter C, Dunst J, Dahl O, Schild SE, Noack F. Prognostic impact of VEGF and VEGF receptor 1 (FLT1) expression in patients irradiated for stage II/III non-small cell lung cancer (NSCLC). Strahlentherapie und Onkologie : Organ der Deutschen Rontgengesellschaft [et al]. 2010 Jun;186(6):307-314.

[62] Samara KD, Antoniou KM, Karagiannis K, Margaritopoulos G, Lasithiotaki I, Koutala E, et al. Expression profiles of Toll-like receptors in non-small cell lung cancer and idiopathic pulmonary fibrosis. International journal of oncology. 2012 May;40(5):1397-1404.

[63] Zegers CM, Rekers NH, Quaden DH, Lieuwes NG, Yaromina A, Germeraad WT, et al. Radiotherapy combined with the immunocytokine L19-IL2 provides long-lasting antitumour effects. Clinical cancer research : an official journal of the American Association for Cancer Research. 2015 Mar 1;21(5):11511160.

[64] Rekers NH, Zegers CM, Germeraad WT, Dubois L, Lambin P. Long-lasting antitumour effects provided by radiotherapy combined with the immunocytokine L19-IL2. Oncoimmunology. 2015 Aug;4(8):e1021541.

[65] Rekers NH, Zegers CM, Yaromina A, Lieuwes NG, Biemans R, Senden-Gijsbers BL, et al. Combination of radiotherapy with the immunocytokine L19-IL2: Additive effect in a NK cell dependent tumour model. 


\section{CHAPTER 9}

Radiotherapy and oncology : journal of the European Society for Therapeutic Radiology and Oncology. 2015 Sep;116(3):438-442.

[66] Carrizosa DR, Gold KA. New strategies in immunotherapy for non-small cell lung cancer. Translational lung cancer research. 2015 Oct;4(5):553-559.

[67] Simone $\mathrm{CB}, 2 \mathrm{nd}$, Burri $\mathrm{SH}$, Heinzerling JH. Novel radiotherapy approaches for lung cancer: combining radiation therapy with targeted and immunotherapies. Translational lung cancer research. 2015 Oct;4(5):545-552.

[68] Li BT, Lou E, Hsu M, Yu HA, Naidoo J, Zauderer MG, et al. Serum Biomarkers Associated with Clinical Outcomes Fail to Predict Brain Metastases in Patients with Stage IV Non-Small Cell Lung Cancers. PloS one. 2016;11(1):e0146063.

[69] Li X, Asmitananda T, Gao L, Gai D, Song Z, Zhang Y, et al. Biomarkers in the lung cancer diagnosis: a clinical perspective. Neoplasma. 2012;59(5):500-507.

[70] Subramaniam S, Thakur RK, Yadav VK, Nanda R, Chowdhury S, Agrawal A. Lung cancer biomarkers: State of the art. Journal of carcinogenesis. 2013;12:3.

[71] Brustugun OT. Hypoxia as a cause of treatment failure in non-small cell carcinoma of the lung. Seminars in radiation oncology. $2015 \mathrm{Apr} ; 25(2): 87-92$.

[72] Yip C, Blower PJ, Goh V, Landau DB, Cook GJ. Molecular imaging of hypoxia in non-small-cell lung cancer. European journal of nuclear medicine and molecular imaging. 2015 May;42(6):956-976.

[73] Trigonis I, Koh PK, Taylor B, Tamal M, Ryder D, Earl M, et al. Early reduction in tumour [18F]fluorothymidine (FLT) uptake in patients with non-small cell lung cancer (NSCLC) treated with radiotherapy alone. European journal of nuclear medicine and molecular imaging. 2014 Apr;41(4):682-693.

[74] Prabhu RS, Hanasoge S, Magliocca KR, Hall WA, Chen SA, Higgins KA, et al. Lymph node ratio influence on risk of head and neck cancer locoregional recurrence after initial surgical resection: implications for adjuvant therapy. Head \& neck. 2015 Jun;37(6):777-782.

[75] Farjah F, Madtes DK, Wood DE, Flum DR, Zadworny ME, Waworuntu R, et al. Vascular endothelial growth factor $\mathrm{C}$ complements the ability of positron emission tomography to predict nodal disease in lung cancer. The Journal of thoracic and cardiovascular surgery. 2015 Oct;150(4):796-803 e792.

[76] Aerts HJ, Velazquez ER, Leijenaar RT, Parmar C, Grossmann P, Carvalho S, et al. Decoding tumour phenotype by noninvasive imaging using a quantitative radiomics approach. Nature communications. 2014;5:4006. 
Societal Impact and Valorisation 


\section{SOCIETAL IMPACT AND VALORISATION}

In this thesis, processes of data gathering, predictive and prognostic inferences derivation from non-invasive sources of information are thoroughly described. These approaches were discussed in the context of a specific oncological disease - lung cancer, but should not be seen as restricted to that, instead as a powerful way of tackling others, as for instance head and neck, rectal or cervical cancer. In this section the possibility of creating value from the knowledge here presented will be discussed, as well as the approaches that could be followed in order to positively impact people's treatments and potentially improve outcomes.

We face these days a great increase in data availability. This "big data" era is opening branches for data collection, analysis and exploration that will definitely shape the way life is known. Medicine is no exception to this trend. On one hand, the large amount of data and its immediate availability requires new techniques for analysis. This inherently leads to a better insight of its underlying information, going beyond classical statistical techniques. More powerful predictive tools are becoming available, enhancing outcome prediction based on the existent sources, by means of using, for instance, machine learning techniques. This is aligned with the aimed personalized care - bringing the right treatment to the right patient. The use of prognostic models is increasing, with many online tools already available:

- http://www.predict.nhs.uk/ is an online tool used to help deciding the ideal course of treatment following breast cancer surgery, based on one's cancer histopathology;

- http://www.cancer.gov/bcrisktool/ allows estimating woman's risk of developing invasive breast cancer;

- https://www.mskcc.org/nomograms a long list of nomograms developed for a great variety of solid tumours, and based on hundreds or even thousands of patients, can be used to predict cancer outcomes or assess disease risk;

- http://www.predictcancer.org/ a platform making available published models developed at Maastro for lung, rectum, head and neck, and endometrium cancer

In this last one, in due time all the models presented in this thesis will be made available for risk probability assessment of individual patients. This shows one of the greatest outcomes of this research - knowledge sharing. This is further complemented with the sharing of data used for models development and validation at https://www.cancerdata.org/. We believe that great gain is obtained in data sharing. First, this will enable others to assess reproducibility, and allows the investigation of other methodologies and hypothesis in same data. Secondly, making data available will improve other institutes validation procedures, and will help overcoming the main drawback of such data-driven approaches: the lack of enough data for both development and validation purposes. Finally, collec- 
tion and standardization of data will be stimulated to validate previously developed models which will also make it possible to update and improve those.

After this general remark, valorisation of the knowledge derived from these two non-invasive sources of information will be further discussed referring to each independently.

\section{PART 1. IMAGING BIOMARKERS}

Comprising the largest segment of the research conducted in the scope of this thesis, imaging biomarkers were devoted 4 chapters in the present work. Main hypothesis behind the complex imaging analysis here explored followed a single premise: more information is nowadays available in medical images, than circumscribed to most common imaging features, popular in published literature, such as tumour volume or maximum intensity measured with FDG uptake in PET scans. Radiomics offers a high throughput of imaging descriptors, capable of characterizing particular regions of interest (commonly tumours, in the Oncology field), by means of shape and size features, first order statistics - regarding pixel values distribution on corresponding histograms, and texture information, based on the arrangement of consecutive and neighbouring pixels.

Standard practice imaging is commonly used for treatment design and dose planning in radiotherapy facilities. In this project it was investigated how imaging can potentially be even more influential on treatments. The use of imaging for precision medicine instead of a one size fits all approach may be a reality within reach following the positive preliminary conclusions presented along this thesis. Nowadays picture archiving and communication systems (PACS) connect images to healthcare systems (Electronic Medical Records - EMR), that ultimately open the branch for integration with clinical decision apps, providing a fast service anywhere, anytime.

With Radiomics one can benefit from the re-use of an existing source of information: the non-invasive character and promise of a fast and cost effective diagnostic tool will always grant imaging to be a reference tool for disease management. In the end patients will in almost all cases be scanned for diagnosis, treatment planning and follow-up purposes. Radiomics is aligned with these data exploiting we are witnessing nowadays, by converting data into information, based on an existing source: imaging.

During the past years, research has been conducted at Maastro and other institutes in different continents, in an attempt to highlight the advantageous use of medical imaging and its thorough investigation. Ever since, the Radiomics approach here explored has resulted in multiple publications (scientific papers, dissertations, etc.), that show that the presented work has great application possibilities. Currently making its transition into a software application for imaging analysis, Radiomics toolbox will soon become available for purchase, sponsored by OncoRadiomics, a spin-off company that made its way from an idea up to a final product - RADIOMICS ${ }^{\text {TM }}$ (http://www.oncoradiomics.com/). 
Strategies for certification are necessary (within Europe (CE marked) and USA (FDA approved)), so that such software could be made available as a plug-in to be integrated in several software packages. Development was performed in a modular way to allow an easy incorporation into treatment planning and diagnostic radiology software from medical companies (RaySearch, Varian, Aquilab, Siemens, Philips, Intuitim and Elekta, for instance). Additionally, it could be made available as software as a service (SaaS) as to provide means for patient stratification and response assessment in pharma-

sponsored clinical trials. Following preliminary evidences demonstrated in chapter 6 of this thesis, applicability of RADIOMICS TM to monitor longitudinal treatment response, is further warranted for DeltaRadiomics ${ }^{\mathrm{TM}}$ applications.

Application of RADIOMICS ${ }^{\mathrm{TM}}$ to other cancer sites will be investigated in collaboration with renowned clinical centres. Marketing strategy for this encompasses publication of peer-reviewed articles and presentations at key conferences. Currently, several world-leading cancer centres are using a research version of RADIOMICS ${ }^{\text {TM }}$ to support its advantageous use, by generating evidence and awareness of mentioned technology.

\section{PART 2. BLOOD BIOMARKERS}

With biopsies not being a possibility for many lung neoplasms, the possibility of inferring tumour's microenvironment from circulating oncoproteins is a powerful alternative. In this thesis we based our blood biomarkers selection to those related to known adverse and intrinsic characteristics of lung tumours: hypoxia, inflammation, tumour load and immunogenic descriptors. This pre selection of markers was based on literature evidence of their prognostic value, documented on at least 100 patients. We made available for the medical community, anonymized patient data comprising almost 400 of patients with up to 12 dissimilar markers (https://www.cancerdata.org/resource/ doi:10.17195/candat.2016.04.1). We expect with this to engage more institutes in performing similar approaches, testing their hypothesis and improving outcomes using blood biomarkers.

Currently, new methodologies are being developed as, e.g. multiplex panel, allowing multiple biomarkers to be measured simultaneously, even ones present in low abundance. These technologies still need to be validated and standardized before introduction in clinical practice but their quick development opens possibilities for, not only, baseline assessment, but also monitoring during and after treatment in a fast and convenient way.

As a general conclusion, two fundamental steps are to be followed in order to bring the research performed to a higher level of beneficial gain to the society:

- Raising awareness on standardized data collection and sharing, will greatly improve the way Medicine is done nowadays, with unlimited benefit expected to be re- 
trieved from several sources of information. This increase on data availability, will as well help develop a superb variety of approaches on data analysis, following different hypothesis.

- Engage more institutes in using Radiomics software implementations, in its research version as to generate evidence of its great usage; and later by its commercialization and integration with medical software platforms. 
Acknowledgments 
First of all, I'd like to thank you, dear reader! Either you made your way to this section after reading all the contents of this thesis, or you skipped it and came straight here, trying to find your name in the following list :-) Anyways you are holding a copy of this book, therefore, thank you!

I would like to dedicate my first words to my supervisor, Philippe. Without his support, his guidance and his patience, this thesis would never have been a reality. Thank you for many times challenging me to think out of the box! Together with him, my copromoters, Cary and Wouter, hold my hand and helped me on this long, sometimes tortuous journey. Cary, for you I have only a great word of appreciation for being a great supervisor and a sweet human being. Sometimes, even when you did not notice it, you shared so much knowledge with me, that I felt I had to carry a basket to collect all the "pearls" you were giving away. Wouter, you are an inspiration, a source of knowledge and a clever mind that everybody looks for guidance and advice. And there is a reason for that!

To my other half team, Ralph(ie), words are not enough to tell you how much I enjoyed working with you. Even when you killed my nerves (and you did... quite often!), our fruitful discussions only made me enrich my own work (and yours, I hope). You are a great scientist, never forget that!

To Hugo and Emmanuel, a big thank you for believing in me and giving me the opportunity to participate in this project and to come to the Netherlands for my PhD, after that skype interview that gave me more adrenaline than a 4-loop roller-coaster!

Even though not having an official role in my PhD, I need to address a special thankful message to Esther Troost. You are so kind and helped me so much and in so many ways that you cannot even imagine! Likewise, I need to tell you, Georgi, that you were a great source of guidance, knowledge and friendship! I really enjoy that even you being far away now, we still share some good moments every now and then.

I want to dedicate a word of appreciation to the members of my reading committee for the time and effort in evaluating my thesis. I deeply thank you, Professor Frank, Professor Anne-Marie, Doctor Eline, Professor Irene and Professor Nuno. To Frank I'd like to extend these words, not only to reflect his commitment in evaluating my thesis, but foremost for creating a fruitful environment amongst the PhD students by encouraging knowledge sharing in the sandwich meetings.

There are many people who made my stay in the Netherlands an unforgettable one. I still remember when I came in the first day of work, after landing the night before in the tiny airport of Maastricht, carrying all I could in two suitcases and a head full of dreams. I remember your kindness in helping me finding an apartment, Shane; I remember you buying me lunch as first day welcoming gesture, Skadi; and I remember quite vividly your big eyes looking at me and asking me questions, trying to make me forget that I was away from home and that I could find here nice friends, Hoda. Five years have passed ever since that day, many of you have entered my life and will remain there forever! 
To my girls: Skadi, Karen and Hoda. Maastricht would not have been the same without you. I love you all so much! I was really fortunate to have found you girls! Those have been memorable years of laughs and tears, babies and dinners, and afternoon shopping activities, weddings, ... Which could have never been complete without our Charlie - Ruud! Even far away, we are always close!

To my three paranynfen (not sure on how to write this word): Shane, after all the Salsa dancing, assembling IKEA furniture and endless Game of Thrones' discussions, you managed to escape this day, by placing yourself on the other side of the World... Unforgivable! Ruben, you came in later, but right on time to grant yourself in a special place in my heart - my dear friend! (do I see a tear?). Aniek, you are just great, an inspiration, and I absolutely love you! So full of life and great adventure stories to tell, and a warming smile! I am going to miss not having someone across the desk who sings along with me the most random songs as you do...I am really happy to have you both by my side on this day! I thank you ahead for that moment in which I will panic a little and you will help me put it back together :-;

Mark and Stefan, you have witnessed my arrival and my departure. You are both amazing and great people! Stefan, thank you for your kindness and nice stories about your amazing trips! Mark, sometimes I get a little lost with your perfect English disguised in that awfully native accent you have, but your eyes never lie, and they show me the great human being you are!

Many people have passed through Maastro during my stay. It is hard to personalize a thankful message to each and any one of you, but I still have good memories of you all: Oana, who started on the very same day; Coumba who was there also doing an internship; Daniela, always on the run and constantly nervous; Guillaume, with his calm yet very deep presence; Lotte, lunch times will never be the same again: what special treat does she bring today for desert?; Patrick and his beautiful and sweet wife Sanaz, it was wonderful to get to know you and share some good moments; Davide, with his very intermittent yet really nice presence; Francesco, that smile and good mood were absolutely great; Jurgen, or one of the Rubens, better than knowing a person's name, is to know the person, right? (I'Il try to stop making the confusion, I swear I will); Adriana, thanks for your nice smile and kindness; Esther van Bloemen, always so kind and sweet; Timo, I'm always surprised that you are so amusing for the German standards ;) Evelyn, great to have someone so reliable as you around; Janita, you arrived in late, but I feel that connecting with you was so easy... felt really natural!; Seán, my dear Seán, those endless stories you have! I'm going to miss those! And your beautiful smile too, Céline! Johan, treat my desk nicely - it comes with the task of taking care of Ralph, but I'm sure you'll manage; Matilde, we shared the same open space for a short yet very nice time; Fiere and Lars, it was always a pleasure to share a smile with you in the corridor! Andre, Erik and Joeri, a big thank you for all the help and even guidance you provided me during these years to retrieve all my data; Nicole, you were always part of our group! Kranthi, I really enjoyed having the chance to get to know you a little better and sharing this 
special day with you! Ellen, you have been since first day and even before an incredible nice person, who helped making my move here a very smooth one! Rianne, great to have you helping me, always with a warm smile!

Please, don't feel like I forgot you case I missed your name in this list. This section is way much harder to write than I anticipated! Not being here listed doesn't mean that you are not in my heart!

Obrigada, Nélson, pela maravilhosa capa que fizeste para a minha tese. Certamente que este pequeno contributo para o Mundo científico ficou mais valorizado com a tua sempre maravilhosa intervenção!

Para os portugueses (de nacionalidade e falantes) que se juntaram: foi uma honra partilhar a estadia em Maastricht convosco. Pedro, como é que alguém anda sempre a correr dessa maneira? Isabel, sempre disponível e com um sorriso, obrigada por aumentares o meu léxico com a palavra Corfbol. Mariana e Joana, acho que Maastricht nunca mais será a mesma, trouxeram demasiada alegria aos imensos dias cinzentos que por cá se vivem... Do outro lado do Atlântico e falando uma versão (des)aprimorada da língua: Gabriel, Louise e Murilo, espero que apreciem tanto esta estadia na Holanda como eu o fiz.

Para os meus amigos em Portugal e espalhados pelo Mundo, a gratidão e amor que vos tenho não pode ficar resumida a palavras... Mas felizmente os atos são muitos: são reencontros inesperados, outros planeados com meses de antecipação e emoção, as conversas online, que nos aproximam tanto! Foi difícil despedir-me de todos quando há 5 anos embarquei nesta jornada, mas a certeza da vossa amizade apenas se tornou mais forte e verdadeira! Um imenso agradecimento! Não vou escrever nomes, que estou debaixo de algum stress e se deixar algum para trás bem sei a repercussão que isso terá até ao fim dos meus dias... Vocês sabem quem são!

À minha família, os meus pilares, palavras são insuficientes para agradecer o apoio, tão fulcral nestes anos em que a distância física só foi suportável pelo imenso amor e carinho que nutrimos uns pelos outros!

Para ti, meu amigo, meu marido, amor da minha vida, meu Mundo... Tu sabes ler no meu silêncio a eterna gratidão que te tenho por estares sempre ao meu lado e me fazeres tão feliz! 
Curriculum Vitae 
Sara João Botelho de Carvalho was born in June $24^{\text {th }} 1985$ in Coimbra, Portugal. She spent her childhood in Grada, and completed high school in Anadia in 2003. The same year she started the Integrated Master in Biomedical Engineer at the Faculty of Sciences and Technology, University of Coimbra, Portugal. Her Master's degree was obtained in 2008, with a grade of 15 out of 20, which final thesis project was entitled "Contribution for the viability study of a PET tomograph, based in RPC (Resistive Plate Chambers) Correction of scattered radiation" with the classification of 18/20 values, under orientation of Nuno Ferreira, PhD. The work group is placed at LIP - Laboratory of Instrumentation and Experimental Physics of Particles, Physics' Department of University of Coimbra. In 2008 she started working at a spin-off company of Critical Software - Critical Health, developing functions of a Software Product Assurance Engineer, for medical software development. Main responsibilities comprised software testing and user requirements assessment for the software development, conducted in a quality controlled environments. She was further deeply involved in medical quality process implementation. In 2010, she joined the Clear PEM project, in the validation procedures of a Positron Emission Tomograph for mammography by means of application of NEMA NU 4-2008 Standards and phantoms' measurements (for image quality assessment). In 2011 she moved in to the Netherlands in order to follow a PhD at Maastro Clinic, that resulted in the work presented in this thesis. It comprised prognostic/predictive modelling based on clinical data, imaging (Radiomics project - www.radiomics.org), and blood biomarkers (clinicaltrials.gov NCT01936571) for oncology applications, particularly lung cancer. Currently she is pursuing a career in the healthcare/pharma sector as a data scientist and preparing a move to Cambridge, United Kingdom. 
List of publications 


\section{LIST OF PUBLICATIONS}

Lambin P, Rios-Velazquez E, Leijenaar R, Carvalho S, van Stiphout RG, Granton P, Zegers CM, Gillies R, Boellard R, Dekker A, Aerts HJ. Radiomics: extracting more information from medical images using advanced feature analysis. Eur J Cancer. 2012 Mar;48(4):441-6. doi: 10.1016/j.ejca.2011.11.036. Epub 2012 Jan 16

Lambin P, Roelofs E, Reymen B, Velazquez ER, Buijsen J, Zegers CM, Carvalho S, Leijenaar RT, Nalbantov G, Oberije C, Scott Marshall M, Hoebers F, Troost EG, van Stiphout RG, van Elmpt W, van der Weijden T, Boersma L, Valentini V, Dekker A. 'Rapid Learning health care in oncology' - an approach towards decision support systems enabling customised radiotherapy'. Radiother Oncol. 2013 Oct;109(1):159-64. doi: 10.1016/j.radonc.2013.07.007. Epub 2013 Aug 28.

Leijenaar RT, Carvalho S, Velazquez ER, van Elmpt WJ, Parmar C, Hoekstra OS, Hoekstra CJ, Boellaard R, Dekker AL, Gillies RJ, Aerts HJ, Lambin P. Stability of FDG-PET Radiomics features: an integrated analysis of test-retest and inter-observer variability. Acta Oncol. 2013 Oct;52(7):1391-7. doi: 10.3109/0284186X.2013.812798. Epub 2013 Sep 9.

Carvalho S, Leijenaar RT, Velazquez ER, Oberije C, Parmar C, van Elmpt W, Reymen B, Troost EG, Oellers M, Dekker A, Gillies R, Aerts HJ, Lambin P. Prognostic value of metabolic metrics extracted from baseline positron emission tomography images in non-small cell lung cancer. Acta Oncol. 2013 Oct;52(7):1398-404. doi: 10.3109/ 0284186X.2013.812795. Epub 2013 Sep 9.

Parmar C, Rios Velazquez E, Leijenaar R, Jermoumi M, Carvalho S, Mak RH, Mitra S, Shankar BU, Kikinis R, Haibe-Kains B, Lambin P, Aerts HJ. Robust Radiomics feature quantification using semiautomatic volumetric segmentation. PLoS One. 2014 Jul 15;9(7):e102107. doi: 10.1371/journal.pone.0102107. eCollection 2014.

Toma-Dasu I, Uhrdin J, Lazzeroni M, Carvalho S, van Elmpt W, Lambin P, Dasu A. Evaluating tumor response of non-small cell lung cancer patients with ${ }^{18} \mathrm{~F}$-fludeoxyglucosepositron emission tomography: potential for treatment individualization. Int J Radiat Oncol Biol Phys. 2015 Feb 1;91(2):376-84. doi: 10.1016/j.ijrobp.2014.10.012.

Panth KM, Leijenaar RT, Carvalho S, Lieuwes NG, Yaromina A, Dubois L, Lambin P. Is there a causal relationship between genetic changes and radiomics-based image features? An in vivo preclinical experiment with doxycycline inducible GADD34 tumor cells. Radiother Oncol. 2015 Sep;116(3):462-6. doi: 10.1016/j.radonc.2015. 06.013. Epub 2015 Jul 7.

Leijenaar RT, Nalbantov G, Carvalho S, van Elmpt WJ, Troost EG, Boellaard R, Aerts HJ, Gillies RJ, Lambin P. The effect of SUV discretization in quantitative FDG-PET Radi- 
omics: the need for standardized methodology in tumor texture analysis. Sci Rep. 2015 Aug 5;5:11075. doi: 10.1038/srep11075.

Leijenaar RT, Carvalho S, Hoebers FJ, Aerts HJ, van Elmpt WJ, Huang SH, Chan B, Waldron JN, O'sullivan B, Lambin P. External validation of a prognostic CT-based radiomic signature in oropharyngeal squamous cell carcinoma. Acta Oncol. 2015;54(9): 1423-9. doi: 10.3109/0284186X.2015.1061214. Epub 2015 Aug 12.

Lambin P, Zindler J, Vanneste B, van de Voorde L, Jacobs M, Eekers D, Peerlings J, Reymen B, Larue RT, Deist TM, de Jong EE, Even AJ, Berlanga AJ, Roelofs E, Cheng $Q$, Carvalho S, Leijenaar RT, Zegers CM, van Limbergen E, Berbee $M$, van Elmpt W, Oberije C, Houben R, Dekker A, Boersma L, Verhaegen F, Bosmans G, Hoebers F, Smits K, Walsh S. Modern clinical research: How rapid learning health care and cohort multiple randomised clinical trials complement traditional evidence based medicine. Acta Oncol. 2015;54(9):1289-300. doi: 10.3109/0284186X.2015.1062136. Epub 2015 Sep 23.

Lambin P, Zindler J, Vanneste BG, De Voorde LV, Eekers D, Compter I, Panth KM, Peerlings J, Larue RT, Deist TM, Jochems A, Lustberg T, van Soest J, de Jong EE, Even AJ, Reymen B, Rekers N, van Gisbergen M, Roelofs E, Carvalho S, Leijenaar RT, Zegers CM, Jacobs M, van Timmeren J, Brouwers P, Lal JA, Dubois L, Yaromina A, Van Limbergen EJ, Berbee $M$, van Elmpt W, Oberije C, Ramaekers B, Dekker A, Boersma LJ, Hoebers F, Smits KM, Berlanga AJ, Walsh S. Decision support systems for personalized and participative radiation oncology. Adv Drug Deliv Rev. 2016 Jan 14. pii: S0169-409X(16)30008-4. doi: 10.1016/j.addr.2016.01.006.

Carvalho S, Troost EG, Bons J, Menheere P, Lambin P, Oberije C. Prognostic value of bloodbiomarkers related to hypoxia, inflammation, immune response and tumour load in non-small cell lung cancer - A survival model with external validation. Radiother Oncol. 2016 Apr 29. pii: S0167-8140(16)31070-2. doi: 10.1016/j.radonc.2016.04.024.

\section{CONFERENCE TALKS}

Carvalho S, Leijenaar R, Rios E, Oberije C, Reymen B, Troost E, Oellers M, Dekker A, Aerts $\mathrm{H}$, Lambin P. Predicting overall survival in NSCLC patients with metabolic metrics derived from baseline PET: a prospective study. Oral communication at $2^{\text {nd }}$ ESTRO Forum, Geneva - Switzerland.

Carvalho S, Leijenaar R, Troost E, Oberije C, Reymen B, van Elmpt W, de Groot M, Bussink J, Dekker A, Lambin P. Prognostic value of pre-Radiotherapy PET metrics of lymph nodes vs. primary tumor in NSCLC - An exploratory study with external validation. Oral communication at European Molecular Imaging Meeting EMIM 2015, Tubingen - Germany. 
Carvalho S, Leijenaar R, Troost E, Oberije C, Reymen B, van Elmpt W, de Groot M, Bussink J, Meldolesi E, Valentini V, Dekker A, Lambin P, Prognostic value of pre-RT PET metrics of lymph nodes vs. primary tumor in NSCLC: which holds more information? Oral communication at $3^{\text {rd }}$ ESTRO Forum, Barcelona - Spain. 


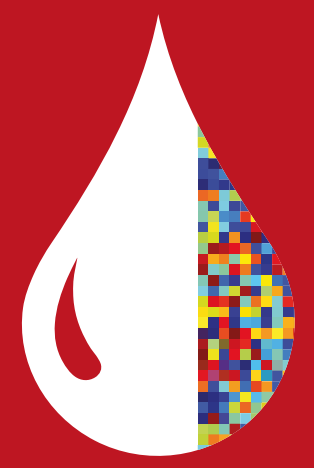

